\title{
A dark field illumination probe linked to Raman spectroscopy for non-invasivety determination of ocular biomarkers
}

Citation for published version (APA):

Zhang, S. (2021). A dark field illumination probe linked to Raman spectroscopy for non-invasivety determination of ocular biomarkers. [Doctoral Thesis, Maastricht University]. Maastricht University. https://doi.org/10.26481/dis.20210707sz

Document status and date:

Published: 01/01/2021

DOI:

10.26481/dis.20210707sz

Document Version:

Publisher's PDF, also known as Version of record

\section{Please check the document version of this publication:}

- A submitted manuscript is the version of the article upon submission and before peer-review. There can be important differences between the submitted version and the official published version of record. People interested in the research are advised to contact the author for the final version of the publication, or visit the DOI to the publisher's website.

- The final author version and the galley proof are versions of the publication after peer review.

- The final published version features the final layout of the paper including the volume, issue and page numbers.

Link to publication

\footnotetext{
General rights rights.

- You may freely distribute the URL identifying the publication in the public portal. please follow below link for the End User Agreement:

www.umlib.nl/taverne-license

Take down policy

If you believe that this document breaches copyright please contact us at:

repository@maastrichtuniversity.nl

providing details and we will investigate your claim.
}

Copyright and moral rights for the publications made accessible in the public portal are retained by the authors and/or other copyright owners and it is a condition of accessing publications that users recognise and abide by the legal requirements associated with these

- Users may download and print one copy of any publication from the public portal for the purpose of private study or research.

- You may not further distribute the material or use it for any profit-making activity or commercial gain

If the publication is distributed under the terms of Article 25fa of the Dutch Copyright Act, indicated by the "Taverne" license above, 


\section{A dark field illumination probe linked to Raman spectroscopy for non-invasively determination of ocular biomarkers}


(C) Shuo Zhang, Maastricht, 2021

All rights are reserved. For articles published, the copyrights have been transferred to the respective publishers. No part of this book may be reproduced or transmitted in any form or by any means, without prior permission in writing by the author, or when appropriate, by the publishers of the publications.

Layout by: Shuo Zhang.

Cover designed by: Christian Bertens \& Shuo Zhang.

Printed by: Gildeprint, Enschede.

ISBN: 9789464192612 


\section{A dark field illumination probe linked to Raman spectroscopy for non-invasively determination of ocular biomarkers}

\section{Dissertation}

to obtain the degree of Doctor at Maastricht University, on the authority of the Rector Magnificus, PROF.DR. RIANNE M. LETSCHERT, in accordance with the decision of the Board of Deans, to be defended in public on WEDNESDAY, $7^{\mathrm{TH}}$ OF JULY 2021 at 16:00 hours

by

Shuo Zhang

born on $18^{\text {th }}$ January,1985 in Shijiazhuang, China 


\section{Supervisors:}

Prof. dr. C.A.B. Webers

Dr. T.T.J.M. Berendschot

\section{Co-supervisor:}

Dr. R.J. Erckens

\section{Assessment committee}

Prof. dr. J. Prickaerts (Chair)

Prof. dr. M.A.M.J. van Zandvoort

Prof. dr. O. Bekers

Prof. dr. J.H. de Boer (UMC Utrecht)

Dr. G.J. Puppels (Erasmus MC)

The studies presented in this thesis were performed at the Department of Ophthalmology, MHeNs School for Mental Health and Neuroscience of Maastricht University. The author is financially supported by the China Scholarship Council (CSC, No. 201309110103). 


\section{Table of Contents}

\section{Chapter Title}

Page

1 Introduction

2 Raman Spectroscopic detection of Interleukin-10 and Angiotensin Converting Enzyme

3 In vitro Raman spectroscopy of sorbitol induced cataracts mimicking diabetic cataracts in porcine lenses

4 Confocal Raman spectroscopy: Evaluation of a noninvasive technique for the detection of topically applied ketorolac tromethamine in vitro and in vivo

4a Pipeline for the removal of hardware related artifacts and background noise for Raman spectroscopy

4b In vitro and in vivo datasets of topically applied ketorolac tromethamine in aqueous humor using Raman spectroscopy

5 Dark field illumination in conjunction with confocal Raman spectroscopy for real time non-invasive aqueous humor investigation

6 Design and performance of a dark field probe with confocal Raman spectroscopy for ophthalmic applications

7 Harnessing abruptly auto-defocusing beam to enhance the Raman signal in aqueous humor: a simulation analysis

8 General Discussion

9 Summary 


\section{Table of Contents}

$\begin{array}{clc}\begin{array}{c}\text { Appendix } \\ \text { I }\end{array} & \text { Title } & \text { Page } \\ \text { II } & \text { Patent } & \text { i } \\ \text { III } & \text { Acknowledgements } & \mathrm{v} \\ \text { IV } & \text { Curriculum Vitae } & \mathrm{xxxv} \\ \text { V } & \text { List of Publications } & \mathrm{xli} \\ & & \mathrm{xlv}\end{array}$




\section{Chapter 1}

Introduction

Shuo Zhang

Page | 1 


\section{Background}

The eye plays a very important role in daily life and dysfunction will severely jeopardize the quality of life. According to a World Health Organization (WHO) report in 2010, worldwide 285 million subjects suffered from visual impairment, including 246 million with low vision (visual acuity between 0.05 and 0.33 ) and 39 million blind (visual acuity less than 0.05 ) due to various causes. ${ }^{1,2}$ The cause of visual impairments ranged from ocular diseases like glaucoma, uveitis or cataracts to systemic diseases like diabetes mellitus or hypertension, or as a result of a traumatic event like a blow to the eye or a penetrating object. Vision is hard to replace by other sensory organs and it is strongly depending on the well-functioning of each part of the eye. Early and effective diagnosis of pathological conditions of the eye is therefore preferred. Furthermore, understanding the normal physiological conditions in the eye is a challenge due to the difficulty of obtaining material from the inside of the eye without compromising its integrity. Fortunately, due to the transparency of eye media, optical techniques are well suited and widely used for this purpose, and there have been promising technical developments in recent years. ${ }^{3}$

In this thesis, we used Raman spectroscopy to obtain information on the chemical components in the anterior chamber of the eye. For this purpose, our research focused on ophthalmic probe developments and data processing.

In the following sections, the anatomy of the eye, eye models and optical techniques to study the individual parts of the eye will be reviewed and discussed.

\section{Anatomy of the eye}

In general, the human visual system consists of three parts, namely the orbit, the adnexa (surrounding tissue) and the eyeball (globe). The orbit refers to the bony part of the skull surrounding the eye to support it and protect it from trauma. The adnexa Extraocular Muscle System (EMS) is not only responsible for following moving objects by moving the eyeballs but also contributes to saccades, the rapid, very small eye movements that are needed for vision. ${ }^{4}$ With contact techniques, neither these saccades nor the eyelids interfere with optical measurements. In general, the exposed region of the globe between the eyelids is $8-11 \mathrm{~mm}$ vertical and $27-30 \mathrm{~mm}$ horizontal in adults. ${ }^{5}$ 


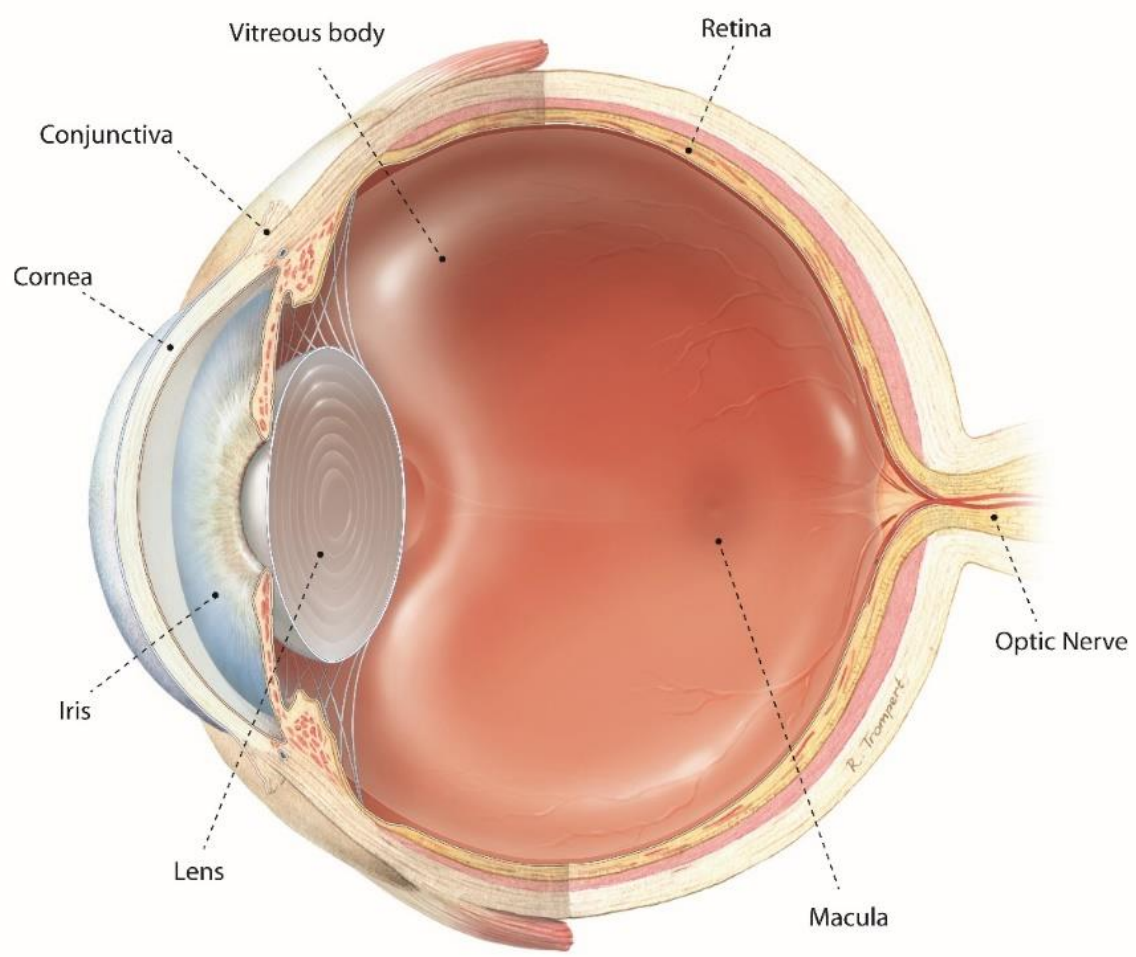

Fig 1 Topographic anatomical structure of the eyeball in sagittal section view. ${ }^{4}$

The average size of the ocular globe for an adult is $23-25 \mathrm{~mm}$ along the optic axis and $24 \mathrm{~mm}$ for transverse diameter, so it is not a true sphere. As shown in Figure 1, the cornea has a different curvature compared to the sclera, the radius being $8 \mathrm{~mm}$ and $12 \mathrm{~mm}$ for the posterior part of the cornea and sclera respectively. In addition, the radius of curvature for the anterior of the cornea differs is slightly smaller, $6.5 \mathrm{~mm}$, than the posterior part. The thickness of the cornea is 540 to $700 \mu \mathrm{m}$ with a mean diameter of the horizontal and vertical meridian of $11.7 \mathrm{~mm}$ and $10.6 \mathrm{~mm}$ for and adult cornea. ${ }^{4}$ Since the sclera is opaque and white it is less suitable to investigate the inner part of the ocular system by optical methods. The optical diagnosis and treatment window is the transparent cornea which covers $1 / 6^{\text {th }}$ of the total surface of the ocular globe. The cornea is the primary refractive element of the eye, which acts like a positive lens providing around 43 diopters (D) in wellfunctioning human eyes. 
The space between the cornea and iris is the anterior chamber (AC), the distance from the inner side of the cornea to the lens is about $3 \mathrm{~mm}$. The $\mathrm{AC}$ is filled with aqueous humor (AH) with a volume of about $200 \mu \mathrm{l}$. AH plays an important role in the physiology of the eye and enables to reveal valuable information of the intraocular system. It provides nutrients, removes the metabolic wastes and helps to maintain proper intraocular pressure (IOP), the latter being a risk factor for glaucoma. AH is produced in the ciliary body by an active filtration process in the epithelial cells to warrant a dynamic balance of the electrolytes in the $\mathrm{AH}$ from plasma. Although the composition between $\mathrm{AH}$ and plasma is very similar, the significant difference is the fact that $\mathrm{AH}$ is devoid of more than $99 \%$ of the plasma proteins, which ensures it acts as a clear and optical transparent medium in the visual pathway. AH mainly contains inorganic ions and organic anions, carbohydrates, glutathione and urea, proteins, growth-modulatory factors, interleukins, oxygen and carbon dioxide. The formation is not static, it is in dynamic equilibrium with a rate of $2 \mu 1 / \mathrm{min}$. The composition ratios and their concentrations reveal the physiology status of the internal ocular system and imbalance of ratios or concentration changes for some specific composition might indicate a dysfunction of the visual system.

The iris consists of blood vessels and connective tissues, among others. Iris tissue is opaque and has a relatively high tolerance for light damage compared to the retina. Light levels at the retina and to some extent imagine quality, can be adapted by controlling the pupil size.

The lens is a transparent tissue without nerve fibers or blood vessels located behind the iris. In adults, the lens diameter is $9-10 \mathrm{~mm}$ in the equatorial direction and its widest anteroposterior thickness is $6 \mathrm{~mm}$. Controlled by ciliary muscles, it can add extra refractive power to focus objects at different distances by shortening its anterior curvature, providing extra 12-16 D. The decrease of its transparency, cataract, is the primary cause of preventable blindness according to a recent report based on the global population who were blind in 2015. ${ }^{6}$ The nutrition of the lens is mainly provided by the AH. Changes therein might help us to predict the cataract in an early stage.

The retina is a sensitive and vulnerable light sensor, which is responsible for converting light information into electrical signals. These electrical signals are transmitted to the brain by the optic nerve. The thickness of the retina is $200-400 \mu \mathrm{m}$ and about $180 \mu \mathrm{m}$ in the central part, the fovea, that is used for fine vision. In this multilayer transparent structure, there are two types of photoreceptors, cones and rods, both sensitive to light. The retina is vulnerable to light damage by high intensity of incident light, ${ }^{8-12}$ which will be discussed further in the section of Limitations for conventional systems and adaption approaches for in vivo applications.

\section{Clinical optics and eye models}

Due to the different structures of the eye and the inhomogeneous optical properties between tissue, it is a challenge to simulate light behavior in the eye. Several eye models 
have been developed that describe the optical properties of the eye with certain boundary conditions. In general, there are two types of eye models designed for different purposes: the Paraxial Model and Finite Wide-Angle Models.

The most common Paraxial one is the Gullstrand eye model and its accommodated versions, like the Exact Gullstrand Eye and Simplified Gullstrand Eye. ${ }^{13}$ As the name already suggests, these paraxial models are suitable for incident light which is parallel or with a small angle along the optic axis of the eye. The model was first introduced in 1909 and has been adapted in the following decades. However, it has limited abilities to provide off-axis aberrations and cannot predict chromatic aberrations. ${ }^{7}$

Therefore, "finite" eye models were developed by several researchers. ${ }^{13-15}$ By adding media dispersion and estimating lens optic properties by a gradient refractive index, the onand off-axis results as well as chromatic aberration were within an acceptable range. Among these models, the Navarro eye model is widely used and well accepted. ${ }^{14,16,17}$ By introducing the dispersion coefficients of ocular media, chromatic aberration is fully taken into account in the Navarro eye model. The dispersion property of each component can be described by the so-called Herzberger formula. ${ }^{18}$

The Liou-Brennan eye ${ }^{19}$ model is another widely known Finite Wide-Angle model. It is adding more modifications and is a further development of the Navarro eye model. Simulation results for visual performance by this model provide better results for refractive surgery. There are two major differences comparing to the Navarro model: first, it is using a 5-degree angle tilt optic axis as the corresponding axis instead of horizontal visual axis in the Navarro model; second, the iris is considered as a circular aperture in front of the front surface of lens and shift $0.5 \mathrm{~mm}$ toward the nasal direction. In addition, the parameters for the radius of cornea curvature, the thickness of the cornea are slightly modified. Figure 2 shows a scheme with parameters for the Liou-Brennan eye model. 


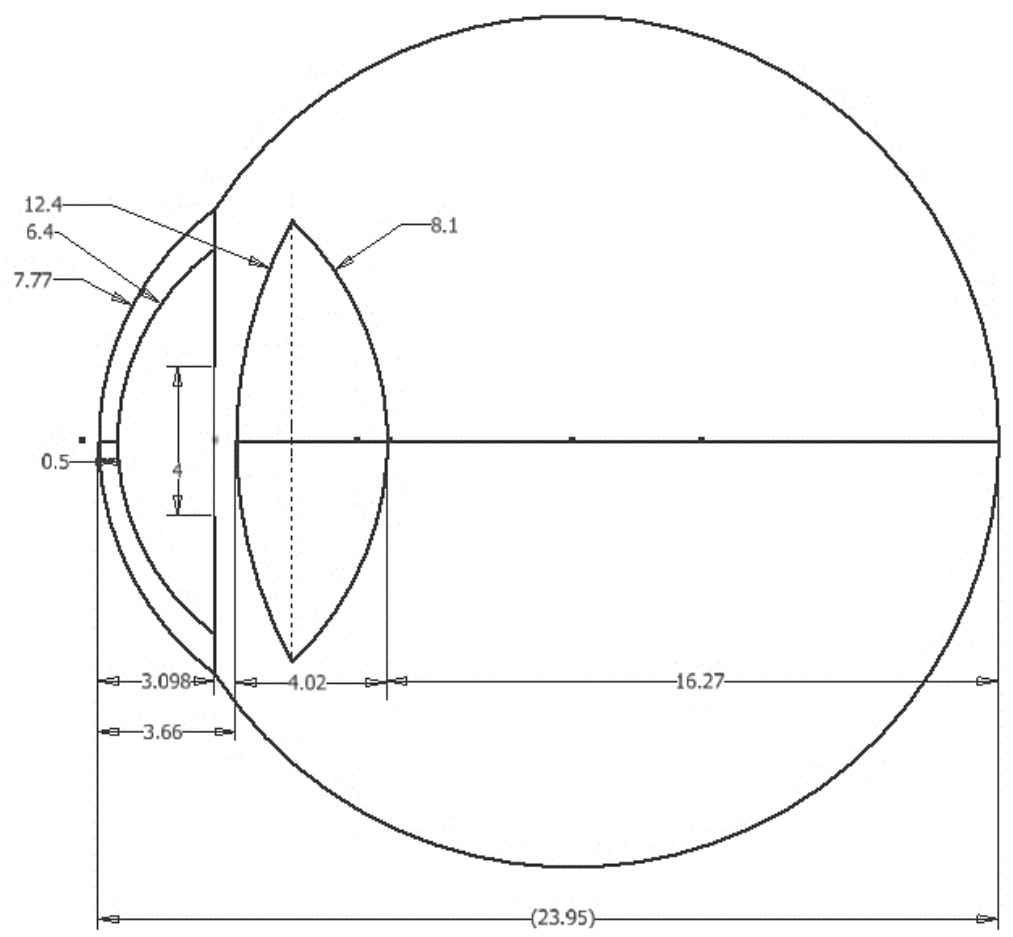

Figure 2 Scheme of Liou-Brennan eye model. The unit is in millimeters.

As shown in Figure 2, the radius of the curvature at the front and back surface of the cornea is $7.77 \mathrm{~mm}$ and $6.40 \mathrm{~mm}$ respectively. The radius at the front and back surface of the lens is $12.40 \mathrm{~mm}$ and $8.10 \mathrm{~mm}$ respectively. Comparing to the anatomy structure introduced previously, the thickness of cornea and AC in this model have slight difference, where we take approximation for $0.50 \mathrm{~mm}$ and $3.16 \mathrm{~mm}$. More detailed parameters can be found in Table 1.

Table 1 Detailed parameters applied in Liou-Brennan eye model. The refractive index of light is at the wavelength of $555 \mathrm{~nm}$ and refractive power is at the condition of relaxed vision.

\begin{tabular}{lcccc}
\multicolumn{1}{c}{ Parameter } & Radius of Curvature $(\mathbf{m m})$ & Thickness $(\mathbf{m m})$ & Refractive Index & Refractive Power(D) \\
\hline Corneal Front Surface & 7.77 & - & 1.376 & - \\
\hline Cornea & - & 0.50 & - & - \\
\hline Corneal Back Surface & 6.40 & - & 1.336
\end{tabular}

Page $\mid 6$ 
In this thesis, we used the Navarro eye model to design and evaluate our ophthalmic device. However, to achieve our goal of having a dark field device, the iris setting of the Liou-Brennan eye mode ${ }^{19}$ was taking into consideration as well.

\section{Major Optic approach}

Optic methods are ideal for non-invasive assessment of the eye where we can distinguish three major types of examination.

- Evaluating the optical properties

- Examining and visualizing the structure

- Determining biological functions of the eye and the molecular profiles within

Diagnostic methods and instruments already cover all these three aspects in clinic practice:

\section{Evaluating the optical properties}

Refractive errors are major causes of visual impairment in the world. To measure refractive errors, several approaches have been developed. Retinoscopy shows its special values in the application on children or patients who are not able to cooperate, however, a great deal of experience is required in clinical practice. To minimize this drawback, the automated refractometer has been developed. In addition, it also can provide sufficient information for lower aberrations situation, but for other types of aberrations like spherical aberration, coma or trefoil, a more precise analysis technique is demanded. Aberrometers fulfilled such requirements properly. ${ }^{7}$

\section{Examining and visualizing the structure}

The slit lamp is the most widely used device for investigating and visualizing the anterior and posterior segment of the eye since first presented by Alvar Gullstrand in 1911. Recent years, Optical coherence tomography (OCT) has been introduced and well developed for the purpose of structure imaging. Combining with adaptive optics (AO), which was initially developed in astronomy, the AO-OCT could provide accurate and precious imaging of the eye with an isotropic resolution of $2-5 \mu \mathrm{m}$ and $5 \mathrm{~mm}$ penetration depth in the eye tissue. ${ }^{7,20}$

\section{Determining biological functions of the eye and the molecular profiles within}

To identify physiology or pathology changes of the eye, information of molecular profile could add extra value for the eye disorders diagnosis in their early stages. For example, blood flow and oxygen saturation alteration of microvascular can be useful indicators of metabolism dysfunctions and disease. Fundus cameras and confocal scanning laser ophthalmoscope could fulfill these requirements and provide powerful supports for ophthalmologists. However, only a limited amount of tests is available by these approaches. 
For the determination of the molecular composition in more detail, Raman spectroscopy may be an option.

\section{Raman Spectroscopy}

In 1928, a new inelastic scattering effect was discovered by the Indian scientist, Sir Chandrasekhara Venkata Raman. ${ }^{26}$ The scattered light shows a wavelength shift compared to the exciting light, in contrast with elastic scattering, or Rayleigh scattering, where there is no such change. Taking a closer look at the light matter interaction on a molecular level, energy is exchanged during the scattering when photons reach a molecule. Molecules will be temporarily excited to a higher virtual energy state and relax back to the initial ground energy state by emitting a photon with a certain amount of energy. This energy exchange may occur in time scales as short as $10^{-15}$ seconds. If the molecules releasing the same amount of energy with incident photons, scattering is called elastic. In this case, no information can be obtained about the particular molecules. In inelastic scattering, the energy released is not equal to the incident photons. Photons can transfer a small amount of energy to the molecule, e.g. to rotations or vibrations of the molecules and the emitted photon show an energy shift that corresponds to this vibrational or rotational energy. This phenomenon is called "Stokes scattering". In contrast, the emitted photon may also gain a small of energy from molecules, which is called "Anti-Stokes scattering". ${ }^{27,28}$ Besides these two main types of Raman scattering, more than 23 other types of Raman scattering have been discovered. ${ }^{29}$

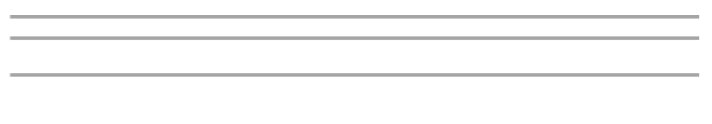

Excited State

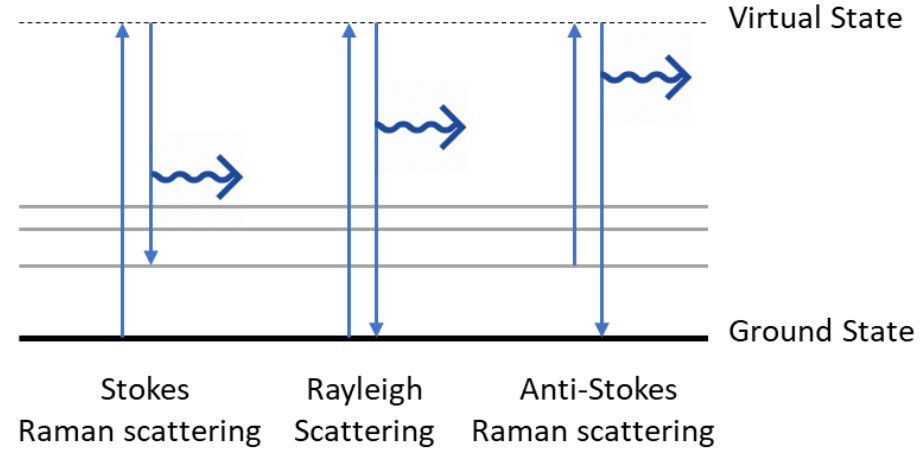

Figure 3 "Jablonski" style diagram of Raman Scattering and Rayleigh scattering. ${ }^{30}$

Page $\mid 8$ 


\section{Raman spectroscopy and variants}

Raman scattering is $10^{6}$ to $10^{8}$ weaker than Rayleigh scattering and therefore needs highly sensitive measuring techniques. The first major breakthrough occurred with the invention of the laser in $1960 .{ }^{31}$ Lasers provide monochromatic and spatial coherent light in combination with high focusing powers, which makes it easy to deliver intense energy of irradiation to samples and distinguish the Raman shift from scattering. Another step forward came from improvements in detection techniques like the use of photomultiplier tubes $^{32}$ and more recent improvement in Charge Coupled Devices (CCD), which were invented in 1970 and introduced for Raman spectroscopy in 1987. ${ }^{33,34}$ Nevertheless, Raman signals are still rather low, and therefore several alternatives have been studied.

Confocal Raman Microscopy. This technique merges microscopy with Raman spectroscopy and as such combining a high spatial resolution ${ }^{35}$ with the ability to uniquely identify molecules by their Raman fingerprint. An example is depicted in Figure 4, which shows a setup for Confocal Raman spectroscopy (CRS). ${ }^{36-38}$

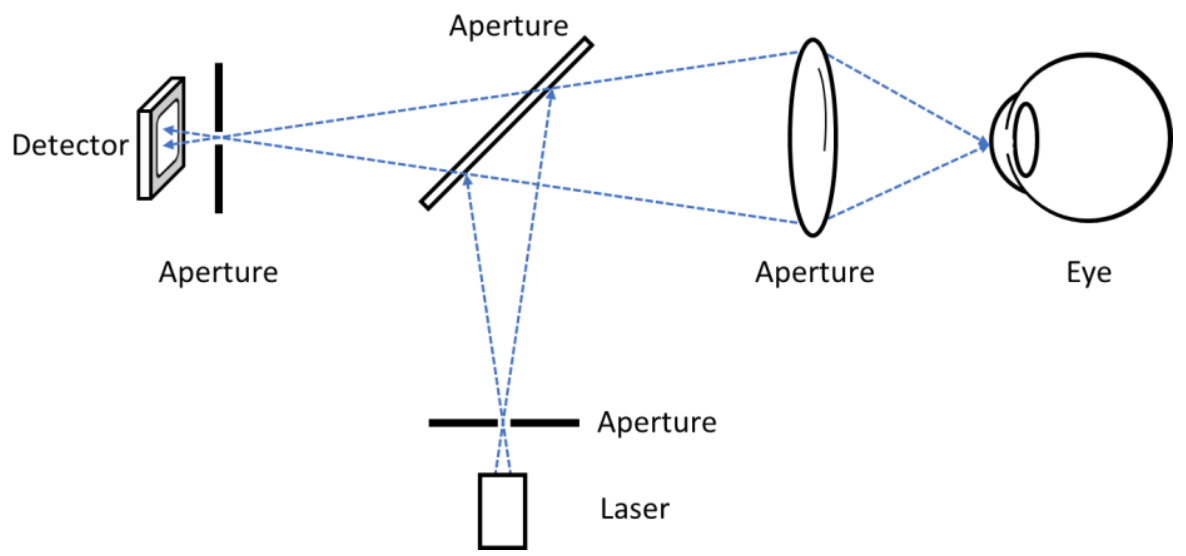

Figure 4 Principle of confocal optics.

The confocal optics use pinholes maintaining a high spatial resolution both in axis and transverse direction. Light out of the focus place will be blocked by the pinholes, resulting in a higher Signal-to-Noise ratio (SNR) compared to a traditional, non-confocal setup. The advantage of this technique in conjunction with Raman spectroscopy is the fact that the optic setup is relatively simple and flexible for application in biological samples. Note however that the Raman signal is not as high as in Resonance Raman scattering and Surface-enhanced Raman scattering (see below).

Resonance Raman scattering (RRS). For some particular molecules and bands, and using with certain wavelength laser, the Raman signal can be enhanced $10^{3}-10^{5}$ fold compared to conventional Raman techniques. ${ }^{27,39,40}$ Unfortunately, it cannot be applied universally since, as mentioned, it only works with particular molecules and selected bands. 
In ophthalmology, applications of RRS are reported on detecting macular carotenoids levels in human eyes, which have been shown to protect against visual loss from age-related macular degeneration. ${ }^{41}$ Another report for the RRS application is the measurement for Amphotericin B concentration in aqueous humor. ${ }^{42,43}$

Surface-enhanced Raman scattering (SERS). Another approach to enhance the Raman signal is Surface-enhanced Raman scattering. Here the Raman signal is detected from a metal surface, most commonly by silver or gold nanoparticles ${ }^{44}$, which results in a $10^{6}$ gain compared to the conventional Raman technique. ${ }^{28}$ If laser light reaches the metal surface, surface plasmons are excited. Hence the Raman signal increases for those molecules with Raman transitions energies close to the energy of the surface plasmons. Although the mechanisms of SERS are still in debate, it is been widely used in biomedical applications. ${ }^{45-47}$ The application of SERS mainly reported on the tear investigation. ${ }^{43,48,49}$ The major obstacle for SERS investigating the intraocular tissue non-invasively is the indispensable metal nanoparticles (NPs) at the target tissue. The biocompatible and nontoxic of the SERS NPs are demonstrated in in vivo tumor targeting and molecular imaging of small animal models. ${ }^{50,51}$ However, in vivo intraocular applications of SERS are still not reported to our knowledge. RRS and SERS can also be combined in so called Surface-enhanced Resonance Raman Spectroscopy (SERRS). ${ }^{52}$

Stimulated Raman scattering (SRS) and Coherent anti-stokes Raman spectroscopy (CARS). In these two approaches, two lasers are used at the same time for stimulated emission of Raman signals. The SNR is around 5 times higher compared to conventional Raman scattering with spontaneous emission. Freudiger et al. demonstrated SRS applications for imaging of brain and skin tissues. ${ }^{53}$ Saar et al. reported that using SRS could reveal dermato-pharmacokinetics information in live mice. ${ }^{54}$ Cheng et al. systematically reviewed SRS applications in imaging of living systems. ${ }^{55}$ Ophthalmic applications of SRS have not been reported to our knowledge. A related technique named Coherent anti-Stokes Raman scattering (CARS) has been reported to be used for several ophthalmic applications. Lei et al. used CARS for imaging trabecular meshwork cells, ${ }^{56}$ Ammar et al. imaging the intact mouse cornea, ${ }^{57}$ Evans et al. demonstrated the possibilities of CARS to obtain the full three-dimensional structure of the fixed human retina. ${ }^{58}$ The two direct illuminated laser requirements limited the in vivo applications of SRS and CARS, due to the potential light hazard possibilities of ocular tissues. For selected chemicals, these techniques allow acquisition at video rates. ${ }^{59}$

\section{Raman spectroscopy applications in ophthalmology}

The ability of Raman spectroscopy to identify and quantify molecules enabled its role in variant biomedical applications in clinical practices ${ }^{60-62}$, including ophthalmology. ${ }^{63}$ Erckens et al. demonstrated the possibility of Raman technique for identifying three materials of polymethyl methacrylate (PMMA), acrylic and silicone as an intraocular lens in patient ${ }^{37}$ and for in vivo evaluating drug induced cornea hydration changes by continues 
monitoring water content in rabbit eyes. ${ }^{64}$ Elshout et al. obtained Raman spectra of 29 common used ocular drugs in clinic practices. ${ }^{65}$ Hosseini quantified the local concentration of intraocular drugs used for endophthalmitis by Raman spectroscopy. ${ }^{66}$ Bauer et al. investigated ocular pharmacokinetics in rabbit eyes by a confocal Raman spectroscopy system. ${ }^{36}$

Besides its use to detect ocular drugs and water content it can also be employed to detect particular molecules and to discriminate between normal and diseased states. Kaji et al. showed the capability of visualizing and localizing collagen fibers, proteins, lipids, and DNA/RNA in the rat cornea by coherent anti-Stokes Raman scattering. ${ }^{67}$ Paluszkiewicz et $a l$. reported the Raman spectrum from two human lenses after cataract surgery. They found an excess of tryptophan, tyrosine, phenylalanine, Beta-sheet, the cause being still under discussion. ${ }^{68}$ In comparing healthy and cataractous human lenses with Raman spectroscopy, they found differences in the concentration of Tyr and Trp residues. ${ }^{69}$

Biomarkers for eye diseases like TNF alpha (Tumor necrosis factor alpha), Cytochrome C, Lactate acid, Lysozyme and Vascular Endothelial Growth Factor (VEGF) ${ }^{70-}$

${ }^{73}$ have been shown to be Raman active and their characteristic Raman spectra have been obtained by different research groups. ${ }^{74-79}$

\section{Raman spectrum data processing}

As mentioned above, Raman scattering is weak and obtaining reliable data is a challenge, especially for potential clinical applications. Chemical and physical inhomogeneity of the tissue, disturbances from environment light and operation protocol all can be the source of interference and may lead to biased results or inaccurate interpretation of the spectra. Different approaches can be applied to limit these interferences, both in hardware and software. Standardization of operation protocol and proper instrument design both can improve the robustness of the performance of Raman setups in clinical applications. Bandpass and laser line filters are used to filter unwanted light (environment light and the excitation light) reaching the detector. Further, the excitation wavelength should be properly selected and optimized. In general, a shorter excitation laser wavelength yield a higher intensity of Raman scattering, however, accompanied by autofluorescence which may exceed the Raman signal. Using a longer wavelength decreases the autofluorescence, unfortunately, the Raman scattering intensity drops as well with $4^{\text {th }}$ order of the wavelength. In practice, wavelengths in the visible and near-infrared region, like 532 $\mathrm{nm}, 671 \mathrm{~nm}, 785 \mathrm{~nm}$ and $1064 \mathrm{~nm}$ are widely used. ${ }^{80}$

As mentioned above, one of the major contributions to background noise is intrinsic autofluorescence from biologic tissues. Pre- and post- data processing is employed for background subtraction and in order to get reliable results. Preprocessing focuses on minimizing the instrumental variance and the autofluorescence generated by biological tissues. Calibration procedures are used before performing experiments that generate reference data to be used to correct for unwanted environment stray light and dark noise 
from the detector. Before proceeding to data analysis, these background references are subtracted from the raw data. For autofluorescence background subtraction, more complicated methods are employed. A single polynomial or modified polynomial background fitting with different orders is widely used for biomedical Raman spectrum processing. Zhao et al. ${ }^{81}$ described polynomial methods for autofluorescence background subtraction and developed an automated algorithm with improved performance. By adding an evaluation procedure, the method employs multiple iterations to optimize the fitting output until it reached the given evaluation criteria.

For post-processing, multivariate methods to identify and distinguish the interested features from spectral profiles have been developed. Two post-processing techniques being used in this thesis will be briefly introduced in the following.

Principal components analysis (PCA) is a mathematical treatment to extract maximum variances present in the dataset by reducing a large number of variants into a small number. ${ }^{82}$ The features can be considered as principal components (PC), where the first components express the major contribution to the variability in the data. ${ }^{83,}{ }^{84}$ Several researchers demonstrated the capabilities of the PCA for analyzing Raman data obtained from biomedical applications. ${ }^{83-85}$ Bonnier et al. ${ }^{86}$ showed the capability of PCA methods together with cluster identification to distinguish biochemical differences in the spatial distribution.

Support vector machine (SVM) is a machine learning approach to classify data by enlarging the feature space to hyperspace by kernels function. This method has a particular value for a linearly inseparable data set. With the help of transformation kernel functions, data might be separable in the generated hyperspace. ${ }^{87}$ To apply SVM in data classification, a labeled learning data set is required to train the algorithm. ${ }^{88}$ Several studies investigated the possibilities of applying SVM on analyzing Raman spectra obtained from different biomedical applications. ${ }^{89,} 90$ Recently, Ullah et al. ${ }^{88}$ successfully differentiated Raman spectra between feeding male and female infants mother's milk using SVM methods.

\section{Limitations for conventional systems and adaption approaches for in vivo applications}

Conventional CRS techniques use a laser beam that excites the ocular system along or parallax to the optical axis. However, this light can pass the anterior compartment and reach the retina. ${ }^{63}$ Hence, the maximum permissible exposure (MPE) intensity for the retina needs to be carefully considered. ${ }^{9,} 91$ Since light hazard limitations for retina tissues are rather tight, maximum exposure and laser intensities can only be low, which will limit Raman signal intensities. Further, the different options to boost the Raman signal all have limitations when applying in the ocular system. SERS needs nanoparticles to assistant obtaining a stronger signal while RRS only effective on some particular molecules and selected bonds which is not ideal for a complete diagnosis purpose. To avoid light damage, we therefore used a "dark field illumination" approach, which restricts direct excitation 
light to the AC. Thus, only scattered light can reach the posterior parts through the crystalline lens in the eye.

\section{Computer model and simulation}

Ray tracing software Zemax (version 16.5 Sp3, Zemax LLC., Kirkland, USA) was utilized to design a dark field illumination device and simulate its optic performance. Zemax works by simulating the behavior of light rays when propagating through an optic system, generating relevant information, like focusing qualities, reflections, absorption or energy lost. It includes a commercial library containing optical properties of common materials and commercial lenses. Besides simulation features, it also provides powerful optimizing capabilities.

Unfortunately, ray tracing software is not designed for 3D modelling. Therefore, complementary, 3D modelling software Autodesk inventor (professional version 2018, Autodesk corp., San Rafael, USA) was used. With the help of Autodesk inventor, a design idea could be modelled in $3 \mathrm{D}$, which was then transferred into Zemax to characterize its optics performance.

As such the workflow has been a three-step circulation, basic structure design in 3D modelling, ray tracing simulation and optimization, performance characterization and evaluation.

\section{Outline of the thesis}

In this thesis, the use of Raman spectroscopy to distinguish and quantify different molecules and drugs in ophthalmology biomedical applications is explored. A non-invasive, dark-field probe is designed and validated described for the in vivo determination of the molecular composition of the AC.

Chapter 2 investigates if IL10 and ACE, which have been shown to be reliable biomarkers for main retinal diseases, can also be probed by Raman spectroscopy. We determined their characteristic peaks in their chemical form in a cuvette and the relationship between intensity and concentrations, as this is a prerequisite for future application in vivo measurements.

In Chapter 3, we studied the possibilities of confocal Raman spectroscopy for the detection of sorbitol as a potential biomarker in diabetic cataract development in pig eyes ex vivo. The concentration of sorbitol in a pig lens showed a good correlation to the degree of cataract of the lens via their Raman spectrum.

In Chapter 4, the concentration of ocular drug AcularTM (ketorolac) in the porcine and rabbit aqueous humor were determined by the confocal Raman spectroscopy and compared with the high-performance liquid chromatography (HPLC), which is widely used in the pharmaceutical industry and well accepted by the Food and Drug Administration of the USA. Comparison of Raman spectroscopy and HPLC showed good agreement in the ex vivo experiments, but lack of sensitivity in vivo measurement. 
Chapter 4a describes in detail a method for the removal of hardware related artifacts and background noise for Raman spectroscopy by a self-developed Matlab program in the previous study. The animal model and acquired raw dataset from the study in chapter 4 are presented in Chapter $\mathbf{4 b}$.

In preparation for in vivo experiments, a new dark field probe was designed and manufactured that prevents light damage on the retina.

In Chapter 5, the optical performance of this probe is simulated and evaluated by raytracing software Zemax and manufactured assisted by 3D modeling software Autodesk Inventor. The functional prototyped probe was tested on ex vivo rabbit eyes for proof of principle. However, since the performance of this first probe prototype did not meet our expectations, further development was needed.

Chapter 6 describes and validates a redesigned probe made out of Quartz material. The number of mirrors within this probe is reduced and only consists of a flat mirror instead of the curved or rotationally symmetric mirrors in the previous designs. performance of the redesigned probe largely improved Both in misalignment tolerance and Raman signal collection abilities improved comparing with the previous design.

In Chapter 7, a harnessing abruptly auto-defocusing beam is proposed as a Raman excitation in the AC. The simulation results indicate that the laser intensity decreases sharply after the focusing point in the AC, which will prevent potential light damage on the retina.

Chapter 8 discusses the main finds in this thesis in a general perspective. It also illustrates challenges and future directions for Raman spectroscopy in clinical applications.

Finally, Chapter 9 summarizes each chapter of the thesis and briefly reviews the major findings.

\section{References}

1. D. Pascolini, and S. P. Mariotti, "Global estimates of visual impairment: 2010," The British journal of ophthalmology 96(5), 614-618 (2012).

2. S. Resnikoff et al., "Global data on visual impairment in the year 2002," Bull World Health Organ 82(11), 844-851 (2004).

3. S. H. Yun, and S. J. J. Kwok, "Light in diagnosis, therapy and surgery," Nature Biomedical Engineering 1(1), 0008 (2017).

4. A. s. P. o. t. Eye, Adler's Physiology of the Eye, 11 ed., Saunders (2011).

5. L. M. Levine, "Basic and Clinical Science Course, Section 2: Fundamentals and Principles of Ophthalmology," in Basic and Clinical Science Course, p. 430, American Academy of Ophthalmology (2018-2019).

6. S. R. Flaxman et al., "Global causes of blindness and distance vision impairment 1990-2020: a systematic review and meta-analysis," The Lancet Global Health 5(12), e1221-e1234 (2017).

7. K.-H. D. Michael Kaschke, Michael Stefan Rill, Optical Devices in Ophthalmology and Optometry: Technology, Design Principles and Clinical Applications, wiley (2014).

8. P. International Commission on Non-Ionizing Radiation, "ICNIRPguidelines on limits of exposure to incoherent visible and infrared radiation," Health Physics 105(1), 74-96 (2013).

9. P. International Commission on Non-Ionizing Radiation, "ICNIRP Guidelines on Limits of Exposure to Laser Radiation of Wavelengths between $180 \mathrm{~nm}$ and 1,000 mum," Health Phys 105(3), 271-295 (2013).

10. G. K. Watts, "Retinal hazards during laser irradiation of the iris," The British journal of ophthalmology 55(1), 60-67 (1971). 
11. R. Matthes et al., "Revision of guidelines on limits of exposure to laser radiation of wavelengths between $400 \mathrm{~nm}$ and $1.4 \mathrm{mu}$ m," Health Physics 79(4), 431-440 (2000).

12. J. Wang et al., "Safety of cornea and iris in ocular surgery with 355-nm lasers," J Biomed Opt 20(9), 095005 (2015).

13. D. A. Atchison, "Optical models for human myopic eyes," Vision Res 46(14), 2236-2250 (2006).

14. R. C. Bakaraju et al., "Finite schematic eye models and their accuracy to in-vivo data," Vision Res 48(16), 1681-1694 (2008).

15. R. Navarro, "The Optical Design of the Human Eye: a Critical Review," Journal of Optometry 2(1), 3 18 (2009).

16. R. Navarro, J. Santamaria, and J. Bescos, "Accommodation-dependent model of the human eye with aspherics," J Opt Soc Am A 2(8), 1273-1281 (1985).

17. A. Drauschke, "Comparison of Numerical Eye Models and its Representation within a Mechanical Eye Model," IFAC-PapersOnLine 49(25), 133-138 (2016).

18. M. Herzberger, and C. D. Salzberg, "Refractive Indices of Infrared Optical Materials and Color Correction of Infrared Lenses," J. Opt. Soc. Am. 52(4), 420-427 (1962).

19. H. L. Liou, and N. A. Brennan, "Anatomically accurate, finite model eye for optical modeling,".J Opt. Soc. Am. A. Opt. Image. Sci. Vis. 14(8), 1684-1695 (1997).

20. M. Pircher, and R. J. Zawadzki, "Review of adaptive optics OCT (AO-OCT): principles and applications for retinal imaging [Invited]," Biomedical optics express 8(5), 2536-2562 (2017).

21. T. Theelen et al., "Analysis of visual pigment by fundus autofluorescence," Experimental eye research 86(2), 296-304 (2008).

22. T. T. Berendschot, P. J. DeLint, and D. van Norren, "Fundus reflectance-historical and present ideas," Progress in retinal and eye research 22(2), 171-200 (2003).

23. J. Zhang et al., "Reconnection of Interrupted Curvilinear Structures via Cortically Inspired Completion for Ophthalmologic Images," IEEE Transactions on Biomedical Engineering 65(5), 1151-1165 (2018).

24. T. T. Berendschot et al., "Directional model analysis of the spectral reflection from the fovea and parafovea," J. Biomed. Opt. 15(6), 065005 (2010).

25. S. H. Hardarson, "Retinal Oximetry," Acta ophthalmologica 91(thesis2), 1-47 (2013).

26. C. V. Raman, and K. S. Krishnan, "A New Type of Secondary Radiation," Nature 121(501-502 (1928).

27.

28.

E. Smith, and G. Dent, Modern Raman spectroscopy: a practical approach, John Wiley \& Sons (2013).

E. Le Ru, and P. Etchegoin, Principles of Surface-Enhanced Raman Spectroscopy: and related plasmonic effects, Elsevier (2008).

29. D. A. Long, "The Raman effect: a unified treatment of the theory of Raman scattering by molecules," West Sussex (2002).

30. K. J. I. Ember et al., "Raman spectroscopy and regenerative medicine: a review," npj Regenerative Medicine 2(1), (2017).

31. T. H. Maiman, "Stimulated Optical Radiation in Ruby," Nature 187(493 (1960).

32. R. C. C. Leite, and S. P. S. Porto, "Continuous Photoelectric Recording of the Raman Effect in Liquids Excited by the He-Ne Red Laser*," J. Opt. Soc. Am. 54(8), 981-983 (1964).

33. S. B. Dierker et al., "Characterization of order in langmuir-blodgett monolayers by unenhanced Raman spectroscopy," Chemical Physics Letters 137(5), 453-457 (1987).

34. B. W. S. S. G. E., "Charge coupled semiconductor devices," The Bell System Technical Journal 49(4), 6 (1970).

35. A. Downes, and A. Elfick, "Raman spectroscopy and related techniques in biomedicine," Sensors (Basel) 10(3), 1871-1889 (2010).

36. N. J. Bauer, "Potential application of confocal raman spectroscopy in ophthalmology," in ophthalmology, p. 160, Maastricht university (1999).

37. R. J. Erckens et al., "Noninvasive Raman spectroscopic identification of intraocular lens material in the living human eye," J. Cataract. Refract. Surg. 27(7), 1065-1070 (2001).

38. M. Marvin, "Microscopy apparatus," Marvin, Minsky, United States (1961).

39. R. S. Chao, R. K. Khanna, and E. R. Lippincott, "Theoretical and experimental resonance Raman intensities for the manganate ion," Journal of Raman Spectroscopy 3(2-3), 121-131 (1975).

40. T. G. Spiro, "Resonance Raman spectroscopy. New structure probe for biological chromophores," Accounts of Chemical Research 7(10), 339-344 (1974).

41. P. S. Bernstein et al., "Resonance Raman measurement of macular carotenoids in the living human eye," Arch Biochem Biophys 430(2), 163-169 (2004).

42. W. V. La Via et al., "Measurement of Amphotericin B concentration by Resonant Raman Spectroscopy - a novel technique that may be useful for non-invasive monitoring," Medical Mycology 44(2), 169-174 
(2006).

43. C.-C. Lin, "Review: Raman Spectroscopy - A Novel Tool for Noninvasive Analysis of Ocular Surface Fluid," Journal of Medical and Biological Engineering 30(6), 343 (2010).

44. N. John, and S. George, "Raman Spectroscopy," in Spectroscopic Methods for Nanomaterials Characterization, pp. 95-127 (2017).

45. B. Sharma et al., "SERS: Materials, applications, and the future," Materials Today 15(1-2), 16-25 (2012).

46. S. Laing et al., "Surface-enhanced Raman spectroscopy for in vivo biosensing," Nature Reviews Chemistry 1(8), (2017).

47. S. Schlucker, "Surface-enhanced Raman spectroscopy: concepts and chemical applications," Angew Chem Int Ed Engl 53(19), 4756-4795 (2014).

48. $\quad \mathrm{P}$. $\mathrm{Hu}$ et al., "Drop-coating deposition and surface-enhanced Raman spectroscopies (DCDRS and SERS) provide complementary information of whole human tears," Journal of Raman Spectroscopy 45(7), 565-573 (2014).

49. W.-S. Kim et al., "A low-cost, monometallic, surface-enhanced Raman scattering-functionalized paper platform for spot-on bioassays," Sensors and Actuators B: Chemical 222(1112-1118 (2016).

50. X. Qian et al., "In vivo tumor targeting and spectroscopic detection with surface-enhanced Raman nanoparticle tags," Nat Biotechnol 26(1), 83-90 (2008).

51. S. Keren et al., "Noninvasive molecular imaging of small living subjects using Raman spectroscopy," Proceedings of the National Academy of Sciences of the United States of America 105(15), 5844-5849 (2008).

52. P. Hildebrandt, and M. Stockburger, "Surface-enhanced resonance Raman spectroscopy of Rhodamine 6G adsorbed on colloidal silver," The Journal of Physical Chemistry 88(24), 5935-5944 (1984).

53. C. W. Freudiger et al., "Label-free biomedical imaging with high sensitivity by stimulated Raman scattering microscopy," Science 322(5909), 1857-1861 (2008).

54. B. G. Saar et al., "Imaging drug delivery to skin with stimulated Raman scattering microscopy," Mol Pharm 8(3), 969-975 (2011).

55. J. X. Cheng, and X. S. Xie, "Vibrational spectroscopic imaging of living systems: An emerging platform for biology and medicine," Science 350(6264), aaa8870 (2015).

56. T. C. Lei et al., "Label-free imaging of trabecular meshwork cells using Coherent Anti-Stokes Raman Scattering (CARS) microscopy," Molecular Vision 17(283-84), 2628-2633 (2011).

57. D. A. Ammar et al., "Imaging the intact mouse cornea using coherent anti-stokes Raman scattering (CARS)," Invest Ophthalmol Vis Sci 54(8), 5258-5265 (2013).

58. C. L. Evans, and X. S. Xie, "Coherent anti-stokes Raman scattering microscopy: chemical imaging for biology and medicine," Annu Rev Anal Chem (Palo Alto Calif) 1(883-909 (2008).

59. C. L. Evans et al., "Chemical imaging of tissue in vivo with video-rate coherent anti-Stokes Raman scattering microscopy," Proceedings of the National Academy of Sciences of the United States of America 102(46), 16807-16812 (2005).

60. P. Chen et al., "Bio-Raman spectroscopy: a potential clinical analytical method assisting in disease diagnosis," Analytical Methods 3(6), 1257 (2011).

61. I. Pence, and A. Mahadevan-Jansen, "Clinical instrumentation and applications of Raman spectroscopy," Chem Soc Rev 45(7), 1958-1979 (2016).

62. M. J. Baker et al., "Clinical applications of infrared and Raman spectroscopy: state of play and future challenges," The Analyst 143(8), 1735-1757 (2018).

63. R. J. Erckens et al., "Raman spectroscopy in ophthalmology: From experimental tool to applications in vivo," Lasers Med. Sci. 16(4), 236-252 (2001).

64. R. J. Erckens et al., "Drug-induced corneal hydration changes monitoredin vivo by non-invasive confocal Raman spectroscopy," Journal of Raman Spectroscopy 32(9), 733-737 (2001).

65. M. Elshout et al., "Detection of Raman spectra in ocular drugs for potential in vivo application of Raman spectroscopy," J Ocul Pharmacol Ther. 27(5), 445-451 (2011).

66. K. Hosseini, "Optical Biopsy in the Anterior Segment of the Eye," in Ophthalmology, p. 143, Maastricht University (2008).

67. Y. Kaji et al., "Raman microscopy: a noninvasive method to visualize the localizations of biomolecules in the cornea," Cornea 36 Suppl 1(0), S67-S71 (2017).

68. C. Paluszkiewicz et al., "Analysis of human lenses by Raman microspectroscopy," Acta Physica Polonica A 129(2), 244-246 (2016).

69. C. Paluszkiewicz et al., "Vibrational microspectroscopy analysis of human lenses," Spectrochim Acta A Mol Biomol Spectrosc 188(332-337 (2018). 
70. S. Lauwen et al., "Omics Biomarkers in Ophthalmology," Invest Ophthalmol Vis Sci 58(6), BIO88BIO98 (2017).

71. N. G. Lambert et al., "Risk factors and biomarkers of age-related macular degeneration," Prog Retin Eye Res 54(64-102 (2016).

72. E. Kersten et al., "Systemic and ocular fluid compounds as potential biomarkers in age-related macular degeneration," Surv Ophthalmol (2017).

73. J. J. Kuiper et al., "An ocular protein triad can classify four complex retinal diseases," Sci Rep 7(41595 (2017).

74. P. Matteini et al., "Concave gold nanocube assemblies as nanotraps for surface-enhanced Raman scattering-based detection of proteins," Nanoscale 7(8), 3474-3480 (2015).

75. F. d. B. Souza et al., "Intramuscular lactic acid assessment through Raman spectrography: new perspectives in sports medicine," Revista Brasileira de Medicina do Esporte 9(6), 388-395 (2003).

76. M. Bilal et al., "Lactate based optical screening of dengue virus infection in human sera using Raman spectroscopy," Biomedical optics express 8(2), 1250-1256 (2017).

77. S. Cavalu et al., "Raman and surface enhanced Raman spectroscopy of 2, 2, 5, 5-tetramethyl-3pyrrolin-1-yloxy-3-carboxamide labeled proteins: Bovine serum albumin and cytochrome c," Biopolymers 62(6), 341-348 (2001).

78. J. Ko et al., "SERS-based immunoassay of tumor marker VEGF using DNA aptamers and silicaencapsulated hollow gold nanospheres," Physical Chemistry Chemical Physics 15(15), 5379-5385 (2013).

79. K. Czamara et al., "Unsaturated lipid bodies as a hallmark of inflammation studied by Raman 2D and 3D microscopy," Scientific reports 7(40889 (2017).

80. U. Gala, and H. Chauhan, "Principles and applications of Raman spectroscopy in pharmaceutical drug discovery and development," Expert Opin Drug Discov 10(2), 187-206 (2015).

81. J. Zhao et al., "Automated autofluorescence background subtraction algorithm for biomedical Raman spectroscopy," Appl Spectrosc 61(11), 1225-1232 (2007).

82. B. G. Tabachnick, and L. S. Fidell, Using multivariate statistics, Allyn \& Bacon/Pearson Education (2007).

83. H. Nawaz et al., "Prediction of viral loads for diagnosis of Hepatitis C infection in human plasma samples using Raman spectroscopy coupled with partial least squares regression analysis," Journal of Raman Spectroscopy 48(5), 697-704 (2017).

84. H. J. Byrne et al., "Spectral pre and post processing for infrared and Raman spectroscopy of biological tissues and cells," Chem Soc Rev 45(7), 1865-1878 (2016).

85. L. A. Austin, S. Osseiran, and C. L. Evans, "Raman technologies in cancer diagnostics," The Analyst 141(2), 476-503 (2016).

86. F. Bonnier, and H. J. Byrne, "Understanding the molecular information contained in principal component analysis of vibrational spectra of biological systems," The Analyst 137(2), 322-332 (2012).

87. G. James et al., An introduction to statistical learning, Springer (2013).

88. R. Ullah et al., "Raman spectroscopy combined with a support vector machine for differentiating between feeding male and female infants mother's milk," Biomedical optics express 9(2), (2018).

89. S. Khan et al., "Analysis of dengue infection based on Raman spectroscopy and support vector machine (SVM)," Biomedical optics express 7(6), 2249-2256 (2016).

90. M. Sattlecker et al., "Investigation of support vector machines and Raman spectroscopy for lymph node diagnostics," The Analyst 135(5), 895-901 (2010).

91. J. J. Vos, and D. van Norren, "Retinal damage by optical radiation. An alternative to current, ACGIHinspired guidelines," Clin Exp Optom 88(4), 200-211 (2005). 
Page | 18 


\section{Chapter 2}

Raman Spectroscopic detection of Interleukin-10 and Angiotensin Converting Enzyme

Shuo Zhang, Frederieke A.M. van der Mee, Roel J. Erckens, Carroll A.B. Webers and Tos T.J.M. Berendschot Journal of the European Optical Society -Rapid Publication 2021;17:7 DOI: $10.1186 / s 41476-021-00152-z$ 


\begin{abstract}
In this report we present a confocal Raman system to identify the unique spectral features of two proteins, Interleukin-10 and Angiotensin Converting Enzyme. Characteristic Raman spectra were successfully acquired and identified for the first time to our knowledge, showing the potential of Raman spectroscopy as a non-invasive investigation tool for biomedical applications.
\end{abstract}

\title{
Key words
}

Confocal Raman Spectroscopy, Interleukin-10, Interleukin-21, Angiotensin Converting Enzyme 


\section{Introduction}

Recently, a proof-of-concept for molecular profiling of Interleukin-10 (IL-10), Interleukin-21 (IL-21) and Angiotensin Converting Enzyme (ACE) was reported by Kuiper et al. to classify four important ocular conditions affecting the retina. ${ }^{1} \mathrm{IL}-10$ plays a crucial role in preventing inflammatory and autoimmune pathologies of several diseases. ${ }^{2}$ IL-21 not only has key roles in antitumor and antiviral responses but also has major effects on inflammatory responses. ${ }^{3}$ ACE plays an important role among other things in the immune system and regulation of blood flow. It can be elevated in granulomatous diseases. ${ }^{4}$ Acquiring information about these molecules in ocular system could help in a better understanding of the complex underlying pathophysiology. Kuiper et al. used a multiplex immunoassay based on Luminex technology to measure the proteins. In this flow cytometry based method a fluorescent signature can be detected on bead-based assays. However, to reach the $\mathrm{pg} / \mathrm{mL}$ detection limit, sample preparation is complicated and time consuming. ${ }^{5}$ Other techniques for cytokines detection and quantification can reach such concentrations, like surface enhanced Raman spectroscopy (SERS), ${ }^{6-10}$ high performance liquid chromatography (HPLC) ${ }^{11}$, pyro-concentrator (PC), ${ }^{12,13}$ mass spectrometry (MS) ${ }^{14}$ etc.. Although the sensitivity of these techniques is comparable or higher than methods using immunoassays, they all need a biopsy to acquire the sample invasively and test in vitro. In HPLC and MS the biomolecule from the sample needs to be fed into the instrument and measured via a chemical or physical process. SER requires matrix assist for signal enhancement which limited its non-destructive applications. Similarly, PC accumulate the target biomolecules from a drop of sample on a chip resulting from the electrohydrodynamic effect. ${ }^{7,12}$ In addition, electrochemical based biosensor with the combination of optoelectronic components could realize the chip-based biological monitoring by refractive index change. ${ }^{15,16}$ These techniques meet the detection limit requirements of the biomolecules with low volume sample size, which might be challenging for an in vivo measurement, especially testing the chemical composition inside an intact aqueous environment like aqueous humor. In a clinical setting, a preferred option would be a noninvasive and non-destructive technique. We propose to use Raman spectroscopy as a noninvasive and non-destructive sampling technique. ${ }^{8,17-21}$ In this study, IL-10, IL-21 and ACE samples were tested. The characteristic Raman spectra of IL-10 and ACE are presented for the first time, which may pave the way for the development of a non-invasive molecular evidence-based investigation tool in future.

\section{Methods}

The basic configuration of the confocal Raman system used in this study was described in a prior article. ${ }^{19}$ In short: a $671 \mathrm{~nm}, 21 \mathrm{~mW}$ continuous wave diode laser (Ignis 671, Laser Quantum) was used as excitation source for the wavenumber region between 2500 and $4000 \mathrm{~cm}^{-1}$ and a $785 \mathrm{~nm}, 23 \mathrm{~mW}$ continuous wave diode laser (SM $785 \mathrm{~nm}$, Innovative Photonic Solutions) was used as excitation sources for wavenumber range 
between $350 \mathrm{~cm}^{-1}$ and $1800 \mathrm{~cm}^{-1}$. The two lasers were both connected via single-mode optical fibers to a Raman module (HPRM 2500, River Diagnostics ${ }^{\circledR}$ ) and spectra were recorded in both regions individually. In the current configuration. the microscope objective lens used in the prior work was replaced by an achromatic lens (focal length $=80 \mathrm{~mm}$, Linos). It acts both to focus the laser on the sample and to collect the Raman scattered light. A charge-coupled device camera (operating temperature: $-65^{\circ}$ ), integrated within the spectrometer acquired the spectra with a spectral resolution of $2 \mathrm{~cm}^{-1}$ in both wavenumber regions. Samples of IL-10 were supplied by BD Bioscience (BD 554611), IL-21 by ThermoFisher Scientific (Catalog \# 14-8219-62) and ACE by R\&D systems (Catalog \# 929-ZN-010). The samples were transported on dry ice and, when arrived, immediately stored at $-80^{\circ}$. All samples were solved in filtrated ( 0.22 um filter) $0.1 \%$ bovine serum albumin (BSA) in phosphate buffered saline (PBS). The IL-10 sample contained $10 \mu 1$ with a concentration of $5.0 \mathrm{ng} / \mathrm{ml}$. The IL-21 and ACE sample each had a total volume of $10 \mu \mathrm{l}$ with a concentration of $10.0 \mathrm{ng} / \mathrm{ml}$. All samples were tested in fused silica cuvettes after they were moved from the $-80^{\circ}$ freezer and thawed to room temperature.

Spectra of IL-10 and ACE samples were acquired with 60s integration times. For IL21 the integration time was 300s. For all samples, two sequential spectra with same integration time were obtained at the same focus area to determine the characteristic Raman peaks and rule out disturbances of cosmic rays. The signal-to-noise ratio (SNR) for each sample was calculated by dividing the intensity of the highest signal peak by the sample standard deviation of the spectral region of $1750 \mathrm{~cm}^{-1}$ to $1800 \mathrm{~cm}^{-1}$ in each spectrum, where there is no Raman signal but only spectral noise. ${ }^{22}$ Raman spectra of PBS, $0.1 \%$ BSA and the fused silica cuvettes were obtained using the same experimental conditions for background correction and comparison. 


\section{Results}
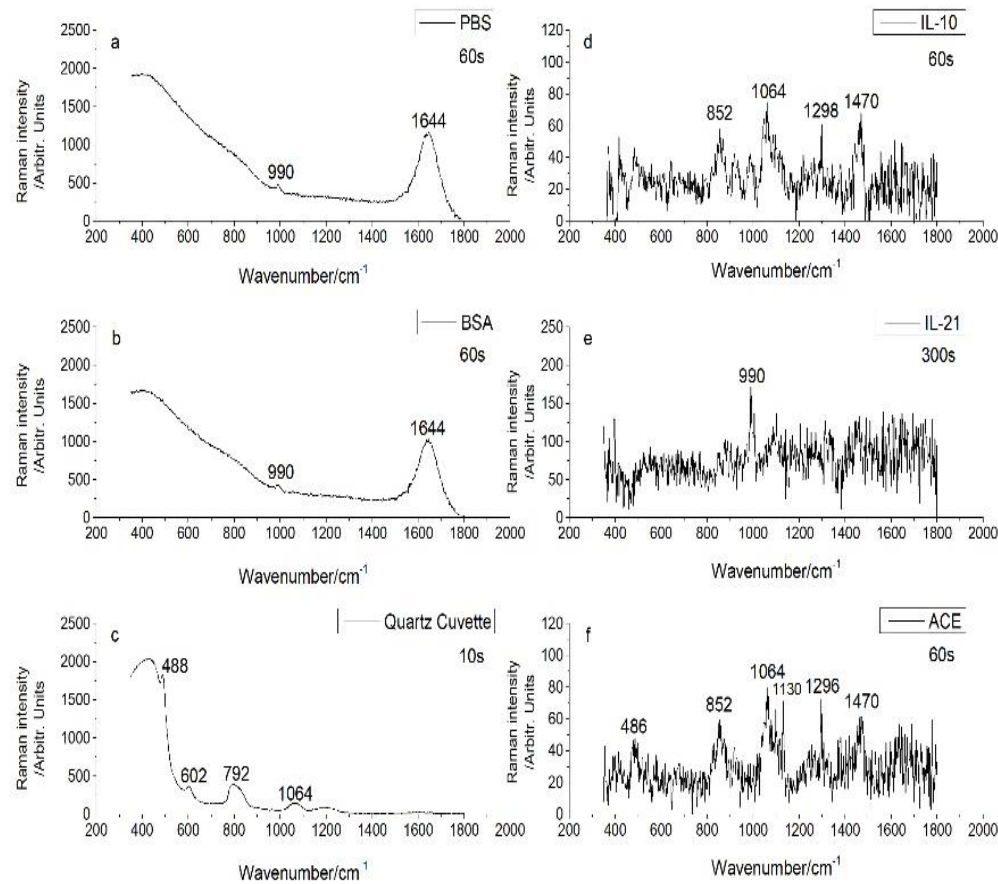

Figure 1. Raman spectra obtained from background reference and samples. Spectrum from background refence of PBS(a), BSA(b), and fused silica cuvette, spectrum of samples from IL-10(d), IL-21(e), and ACE(f).

Figure 1 shows Raman spectra of PBS, BSA, fused silica cuvette, IL-10, IL-21 and ACE. In the wavenumber region between $350 \mathrm{~cm}-1$ and $1800 \mathrm{~cm}^{-1}$, Raman spectra of PBS (Fig. 1a) and BSA (Fig. 1b) both show a peak at $990 \mathrm{~cm}^{-1}$ and a water band at $1644 \mathrm{~cm}^{-1}$. For the fused silica cuvette material (Fig. 1c), bands centered at $430 \mathrm{~cm}^{-1}, 488 \mathrm{~cm}^{-1}, 602$ $\mathrm{cm}^{-1}, 792 \mathrm{~cm}^{-1}$ and $1064 \mathrm{~cm}^{-1}$ can be observed. The Raman band at $430 \mathrm{~cm}^{-1}$ is assigned to symmetric stretching mode of oxygen in a disordered 5- and 6- membered ring of $\mathrm{SiO}_{4}$ tetrahedra. The peaks at 488 and $602 \mathrm{~cm}^{-1}$ are assigned to 4- and 6-membered rings of $\mathrm{SiO}_{4}$ in the random network of the silica glass. ${ }^{23-25}$ The bands centered at 1064 and $1200 \mathrm{~cm}^{-1}$ are attributed, respectively, to transverse-optical and longitudinal-optical components of the three-fold degenerate antisymmetric Si-O-Si stretch mode. ${ }^{24}$ Similarly, the asymmetric band near $792 \mathrm{~cm}^{-1}$ is assigned to the threefold-degenerate "rigid cage" vibrational mode of $\mathrm{SiO}_{2}$ units. ${ }^{24}$ For IL-10 (Fig. 1d) we found peaks at 852, 1064, 1298 and $1470 \mathrm{~cm}^{-1}$. The peak at $852 \mathrm{~cm}^{-1}$ might be from proline, hydroxyproline and tyrosine, $1298 \mathrm{~cm}^{-1}$ is suspected from $\mathrm{CH} 2$ deformation modes and $1470 \mathrm{~cm}^{-1}$ can be assigned to a $\mathrm{C}=\mathrm{N}$ stretching bond within the IL-10 protein structure. ${ }^{9}$ For the spectra of IL-10, the SNR of highest peak at $1064 \mathrm{~cm}^{-1}$ as 5.02. Only a single peak at $990 \mathrm{~cm}^{-1}$ is found in IL-21 spectra (Fig. 1e) with SNR of 6.10. We did not find any identifiable Raman peaks within the high wavenumber region 
between 2500 and $4000 \mathrm{~cm}^{-1}$, except for the broad water band between 3200 and $3500 \mathrm{~cm}^{-1}$ from the solvent. ACE (Fig. 1f) showed peaks at 486, 852, 1064, 1130,1296 and $1470 \mathrm{~cm}^{-1}$. The peak at $852 \mathrm{~cm}^{-1}$ is from single bond stretching vibrations of the amino acids, valine, polysaccharides and tyrosine (Fermi resonance of ring fundamental and overtone) ${ }^{26,27}$ The peak at $1130 \mathrm{~cm}^{-1}$ may be assigned to $\mathrm{C}-\mathrm{C}$ skeletal stretch transconformation, whereas the peak at $1296 \mathrm{~cm}^{-1}$ comes from $\mathrm{CH}_{2}$ deformation. ${ }^{28}$ However, some peaks can also be seen in cuvette results, like the peaks at 600, 792 and $1064 \mathrm{~cm}^{-1}$. The SNR of highest peak at $1064 \mathrm{~cm}^{-1}$ in ACE spectra is 6.15 .

\section{Discussion}

To our knowledge, this is the first report of characteristic Raman spectra of IL-10 and ACE. For IL-10 we found characteristic Raman peaks at 852, 1296 and $1470 \mathrm{~cm}^{-1}$. ACE is characterized by peaks located at $852,1130,1296$ and $1470 \mathrm{~cm}^{-1}$. The peak location at 486 $\mathrm{cm}^{-1}$ can be considered the same from fused silica cuvette peak at $488 \mathrm{~cm}^{-1}$, since the spectral resolution of the spectrometer is $2 \mathrm{~cm}^{-1}$. The peak observed at $990 \mathrm{~cm}^{-1}$ in IL-21 is most probably be attributed to a Raman signal from the additives of PBS. ${ }^{29}$ We did not find additional peaks in the high wavelength region besides the water band.

For validating the characteristic peaks from the IL-10, IL-21 and ACE samples, background spectra from PBS, BSA were acquired. For error estimation between measurements, we calculated the coefficient of variation relative standard deviation (CVRSD) of each sample. ${ }^{30}$ The CVRSD of BSA, PBS, IL-10, ACE and IL-21 are $0.89 \%$, $0.73 \%, 1.68 \%, 1.68 \%$ and $1.25 \%$, respectively. The peak at $990 \mathrm{~cm}^{-1}$ can be found both in PBS and BSA, which indicates no extra peaks will be picked up in the given concentration of our BSA samples. Hence, the $990 \mathrm{~cm}^{-1}$ peak found at IL-21 with $300 \mathrm{~s}$ integration time is most probably from PBS even the background already subtracted, due to the slightly variance introduced by long integration times. Spectra from the fused silica cuvette were obtained by shifting the laser focus onto cuvette itself. Fused silica shows several Raman peaks, one of which, at $1064 \mathrm{~cm}^{-1}$, that was observed in the spectra of IL-10 and ACE. Although the Raman signal intensity is relativity high with $10 \mathrm{~s}$ integration time when focusing on the cuvette material, the use of the confocal system ensures only a minimal contribution from cuvette when focusing at the sample within the cuvette. We analyzed multiple spectra acquired from each sample. The repeatability of the results can be further improved by measuring more individual samples, which unfortunately were not available in the study.

Biological fluids are complex mixtures, which contain variants compounds, ${ }^{31}$ which may have multiple intra-molecular bonds in common. In our case, IL-10 and ACE share peaks at 852,1296 and $1470 \mathrm{~cm}^{-1}$. Only using one single peak to distinguish the proteins of interest from the diverse array of other proteins and other molecules present might lead to a biased conclusion. The $1130 \mathrm{~cm}^{-1}$ peaks only exist in ACE spectra and thus is helpful to differentiate ACE from IL-10. An even more complicated situation originates if each peak 
in the obtained spectra is an integrated result from all compounds it contains. In this case, more advanced data processing techniques are needed. Several technique are proposed for pre- and post-processing Raman data, however a well-accepted standard procedure is still lacking. ${ }^{32}$ While testing biomolecules, the major challenge of Raman spectroscopy is its low detection limit. In addition, for low concentration samples, the SNR might below the limit of quantitation due to the fluorescence interference. A technique was proposed which requires a wavelength tunable laser for sequentially shifting of excitation wavelength, ${ }^{11}$ however, having a fixed wavelength excitation laser, we were unable to use this approach. An alternative approach is to use peak intensity ratios of each compound to identify each compound independently and quantify its concentration. This peak intensity ratio approach already has been reported and validated for ex vivo Raman investigation in human aqueous humor. ${ }^{33}$ In a previous study, we developed a tailored MATLAB (Version 2017b, The Mathworks Inc., Natick, MA, United States) data processing program for in vitro and in vivo Raman detection of ocular drug topically delivered to the animal eyes ${ }^{34,35}$ and demonstrated that with proper data processing, the molecular information can be extracted non-invasively from biological fluids in-situ and ex vivo.

\section{Conclusions}

Raman spectra of IL-10 and ACE were successfully obtained by our confocal Raman system. The SNR of Raman spectra could be further improved. The identification of IL-10 and ACE characteristic Raman peaks shows the potential possibilities for non-invasive investigation in the molecular level, which might help us better understanding the complex underlying the pathophysiology of diseases.

\section{Abbreviations}

IL-10: Interleukin-10; IL-21: Interleukin-21; ACE: Angiotensin Converting Enzyme; MS: Mass Spectrometry; BSA: Bovine Serum Albumin; PBS: Phosphate Buffered Saline; SNR: Signal-to-Noise Ratio; CVRSD: Coefficient Of Variation Relative Standard Deviation.

\section{Availability of data and materials}

Upon request to the authors.

\section{Competing interests}

The authors declare that they have no competing interests.

\section{Funding}

S. Zhang acknowledges the China Scholarship Council for the support by State Scholarship Fund No. 201309110103. 


\section{Authors' contributions}

All authors conceived of and designed the experimental protocol. SZ and FM performed the experiment and collected the data. All authors were involved in the analysis and interpretation of the data. SZ wrote the draft of the manuscript. FM, RE, CW, TB reviewed and revised the manuscript and produced the final version. All authors read and approved the final manuscript.

\section{Acknowledgements}

We thank M. Tempelman from the Translational Immunology department of University Medical Center Utrecht for providing the IL-10, IL-21 and ACE samples. 


\section{Reference:}

1. J. J. Kuiper et al., "An ocular protein triad can classify four complex retinal diseases," Sci Rep 7(41595 (2017).

2. S. S. Iyer, and G. Cheng, "Role of interleukin 10 transcriptional regulation in inflammation and autoimmune disease," Crit Rev Immunol 32(1), 23-63 (2012).

3. R. Spolski, and W. J. Leonard, "Interleukin-21: a double-edged sword with therapeutic potential," Nat Rev Drug Discov 13(5), 379-395 (2014).

4. $\quad$ K. E. Bernstein et al., "Angiotensin-converting enzyme in innate and adaptive immunity," Nat Rev Nephrol 14(5), 325-336 (2018).

5. W. de Jager et al., "Improved multiplex immunoassay performance in human plasma and synovial fluid following removal of interfering heterophilic antibodies," J Immunol Methods 300(1-2), 124-135 (2005).

6. W. E. Smith, "Practical understanding and use of surface enhanced Raman scattering/surface enhanced resonance Raman scattering in chemical and biological analysis," Chem Soc Rev 37(5), 955-964 (2008).

7. M. Pannico et al., "Direct printing of gold nanospheres from colloidal solutions by pyroelectrohydrodynamic jet allows hypersensitive SERS sensing," Applied Surface Science 531((2020).

8. C. C. Pelletier, J. L. Lambert, and M. Borchert, "Determination of glucose in human aqueous humor using Raman spectroscopy and designed-solution calibration," Appl. Spectrosc. 59(8), 1024-1031 (2005).

9. Z. Movasaghi, S. Rehman, and I. U. Rehman, "Raman spectroscopy of biological tissues," Applied Spectroscopy Reviews 42(5), 493-541 (2007).

10. Z. Li et al., "A plasmonic staircase nano-antenna device with strong electric field enhancement for surface enhanced Raman scattering (SERS) applications," Journal of Physics D: Applied Physics 45(30), (2012).

11. S. Marshall, and J. B. Cooper, "Quantitative Raman Spectroscopy when the Signal-to-Noise is Below the Limit of Quantitation due to Fluorescence Interference: Advantages of a Moving Window Sequentially Shifted Excitation Approach," Appl Spectrosc 70(9), 1489-1501 (2016).

12. S. Grilli et al., "Active accumulation of very diluted biomolecules by nano-dispensing for easy detection below the femtomolar range," Nature communications 5(5314 (2014).

13. R. Rega et al., "Detecting Collagen Molecules at Picogram Level through Electric Field-Induced Accumulation," Sensors (Basel) 20(12), (2020).

14. H. Kupcova Skalnikova et al., "Advances in Proteomic Techniques for Cytokine Analysis: Focus on Melanoma Research," Int J Mol Sci 18(12), (2017).

15. K. Xu et al., "Micro optical sensors based on avalanching silicon light-emitting devices monolithically integrated on chips," Optical Materials Express 9(10), (2019).

16. K. Xu et al., "Light emission from a poly-silicon device with carrier injection engineering," Materials Science and Engineering: B 231(28-31 (2018).

17. R. J. Erckens et al., "Raman spectroscopy in ophthalmology: From experimental tool to applications in vivo," Lasers Med. Sci. 16(4), 236-252 (2001).

18. R. J. Erckens et al., "Drug-induced corneal hydration changes monitoredin vivo by non-invasive confocal Raman spectroscopy," Journal of Raman Spectroscopy 32(9), 733-737 (2001).

19. M. Elshout et al., "Detection of Raman spectra in ocular drugs for potential in vivo application of Raman spectroscopy," J Ocul Pharmacol Ther. 27(5), 445-451 (2011).

20. Y. Kaji et al., "Raman microscopy: a noninvasive method to visualize the localizations of biomolecules in the cornea," Cornea 36 Suppl 1(0), S67-S71 (2017).

21. C. Paluszkiewicz et al., "Analysis of human lenses by Raman microspectroscopy," Acta Physica Polonica A 129(2), 244-246 (2016).

22. J. C. Lazaro et al., "Optimizing the Raman signal for characterizing organic samples: The effect of slit aperture and exposure time," Spectrosc-Int J 23(2), 71-80 (2009).

23. K. Awazu, and H. Kawazoe, "Strained Si-O-Si bonds in amorphous SiO2 materials: A family member of active centers in radio, photo, and chemical responses," Journal of Applied Physics 94(10), 6243$6262(2003)$.

24. F. L. Galeener, "Band limits and the vibrational spectra of tetrahedral glasses," Physical Review B 19(8), 4292-4297 (1979).

25. F. L. Galeener, "Planar rings in vitreous silica," Journal of Non-Crystalline Solids 49(1), 53-62 (1982).

26. M. Gniadecka et al., "Diagnosis of basal cell carcinoma by Raman spectroscopy," Journal of Raman 
Spectroscopy 28(23), 125-129 (1997).

27. G. Shetty et al., "Raman spectroscopy: elucidation of biochemical changes in carcinogenesis of oesophagus," British journal of cancer 94(10), 1460-1464 (2006).

28. E. O. Faolain et al., "A study examining the effects of tissue processing on human tissue sections using vibrational spectroscopy," Vibrational Spectroscopy 38(1), 121-127 (2005).

29. C. David et al., "Raman and IR spectroscopy of manganese superoxide dismutase, a pathology biomarker," Vibrational Spectroscopy 62(50-58 (2012).

30. F. J. H. Douglas A. Skoog, Stanley R. Crouch, Principles of Instrumental Analysis, 7th ed., Cengage Learning (2016).

31. L. M. Levine, "Basic and Clinical Science Course, Section 2: Fundamentals and Principles of Ophthalmology," in Basic and Clinical Science Course, p. 430, American Academy of Ophthalmology, (2018-2019).

32. H. J. Byrne et al., "Spectral pre and post processing for infrared and Raman spectroscopy of biological tissues and cells," Chem Soc Rev 45(7), 1865-1878 (2016).

33. S. Woong Moon et al., "Label-free optical detection of age-related and diabetic oxidative damage in human aqueous humors," Microsc Res Tech 79(11), 1050-1055 (2016).

34. C. J. F. Bertens et al., "Confocal Raman spectroscopy: Evaluation of a non-invasive technique for the detection of topically applied ketorolac tromethamine in vitro and in vivo," Int. J. Pharm. 570(118641 (2019).

35. S. Zhang et al., "In vitro and in vivo datasets of topically applied ketorolac tromethamine in aqueous humor using Raman spectroscopy," Data Brief $27(104694$ (2019). 


\section{Chapter 3}

In vitro Raman spectroscopy of sorbitol induced cataracts mimicking diabetic cataracts in porcine lenses

Shuo Zhang" ${ }^{\#}$, Qi Qiao", Roel J. Erckens, Tos T.J.M. Berendschot and Carroll A.B. Webers \# Equal contributing Biomedical Optics Express, Submitted 


\begin{abstract}
One hypothesis of diabetic cataracts suggests that the polyol pathway plays a key role in its development where extra sorbitol is produced and accumulates in the lens leading to the up-regulation of osmotic stress. To investigate this hypothesis sorbitol induced cataracts were measured non-invasively by Raman spectroscopy. Porcine lenses were submerged in PBS diluted sorbitol with concentrations of $2 \mathrm{mM}$ to $50 \mathrm{mM}$ to mimic various grades of diabetic cataractous lenses. The grades of the cataractous lenses were determined by the ratio between protein peak and water peak intensities. Sorbitol concentrations in the treated lens were measured by its Raman peak at $876 \mathrm{~cm}^{-1}$. Linearity of the sorbitol concentrations in the lenses was calculated with R-square of 0.874 and Pearson's R of 0.946 ; the grades of the cataractous lens were with R-square of 0.792 and Pearson's R of 0.907 , respectively. The Pearson correlation between actual concentration in lenses and estimated sorbitol concentration measured by Raman spectroscopy was $0.946(\mathrm{p}=0.01)$ and $0.823(\mathrm{p}=0.023)$ between estimated sorbitol concentration and the cataract grade. This indicates that Raman spectra can quantitively determine sorbitol concentrations in a clinically relevant detection limit and correlate with mimicked diabetic cataract lenses. This study displays the potential of Raman spectroscopy as a non-invasive diagnostic tool in distinguishing diabetic cataracts during early stages and assisting ophthalmologists in providing early medical interventions.
\end{abstract}

\title{
Key words
}

Diabetic cataract, Raman Spectroscopy, Porcine Eye, Sorbitol 


\section{Introduction}

Diabetes mellitus has been a global pandemic threat not only due to hyperglycemia itself, but also from the accompanied complications. Cataract as an ocular complication is a major cause of visual impairment in diabetic patients and has become a worldwide social health and economic burden. ${ }^{1}$ The prevalence of cataracts in the United States was reported to be $76.5 \%$ in adults with diabetes mellitus. ${ }^{2,3}$ Incidences of diabetic cataracts are still increasing while pathophysiological mechanisms of early diabetic cataracts are still not fully understood. A number of mechanisms have been proposed, such as the polyol pathway, increased hexosamine pathway, protein kinase $\mathrm{C}$ pathway, and advanced glycation end products pathway. ${ }^{4,5}$ Moreover, it has been suggested that the polyol pathway plays a key role in diabetic cataract development. ${ }^{1,6-8}$ It is known that diabetes patients are characterized by chronic hyperglycemia that stimulates aldose reductase (AR) resulting in morbid glucose metabolism by activation of the polyol pathway instead of being metabolized through glycolysis. ${ }^{1}$ Therefore, extra sorbitol is accumulated as it is difficult for it to diffuse through membranes, leading to the up-regulation of osmotic stress and resulting in extensive swelling of cortical lens fibers that ultimately degenerate and form diabetic cataracts. ${ }^{7}$ Altogether, gradually increased osmotic stress due to accumulation of extra sorbitol and oxidative stress from decreases in NADPH/NADP+ ratios are a major factor behind the development of diabetes cataracts (Fig. 1). ${ }^{5}$

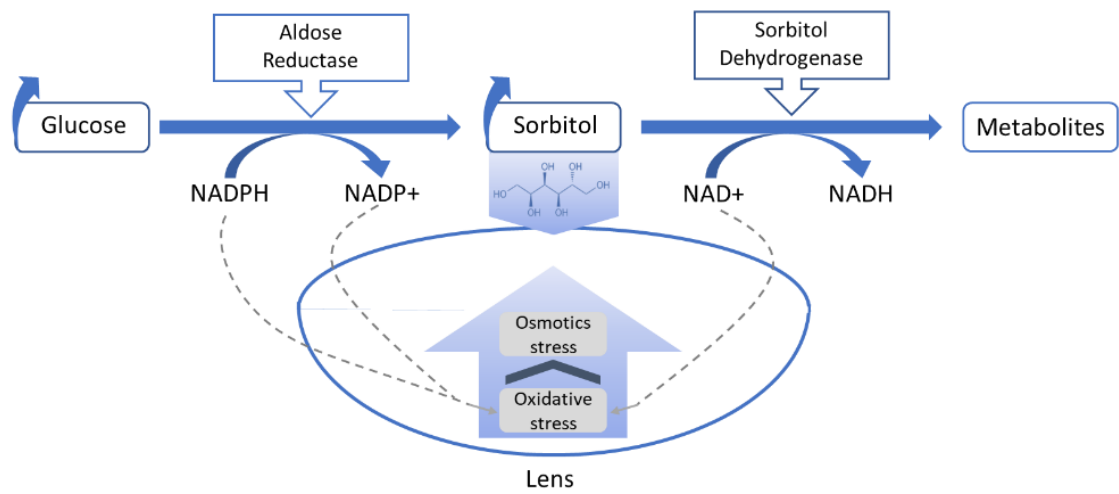

Fig. 1 Polyol pathway in diabetic cataract development. Chronic hyperglycemia actives AR and sorbitol dehydrogenase causing sorbitol and subsequent polyol metabolites to be produced. As accumulated sorbitol is difficult to diffuse through the membranes an up-regulation of osmotic stress and oxidative stress occurs. These combined factors contribute to the extensive swelling of the cortical lens fibers that degenerate and form diabetic cataracts.

An increasing number of publications support the hypothesis that the initiating mechanism in diabetic cataract formation is the generation of polyols from glucose by AR. ${ }^{5}$, ${ }^{7,9}$ While plasma glucose concentrations in diabetes and non-diabetes have already been well established, sorbitol still needs to be further investigated. In an in-vitro study, 
Yoshioka et al. ${ }^{10}$ indicated that sorbitol is 20 times higher in human diabetic cataract lens tissues compared with non-diabetic lens tissues. This accumulation of sorbitol could result in 'fast' development of lens opacification' making sorbitol an optimal indicator in the early diagnosis of diabetic cataract development. Evaluation of sorbitol concentration might reveal underlying mechanisms and provide valuable information for medical interventions. Sorbitol has been studied in different animal models. Varying sorbitol levels in lenses of 10 species were reported by $\mathrm{Kuck}^{11}$ and Jedziniak et al.. ${ }^{12}$ Rabbit, rat and porcine are more often used in sorbitol studies, however, sorbitol levels in rabbit and rat lenses are one magnitude higher than those found in human lenses. ${ }^{11}$ In addition, Malone et al. found in a diabetic rat lens that sorbitol increased but lens hydration did not, ${ }^{13}$ which is different from the human lens. Vaca et al. examined sorbitol dehydrogenase levels in several porcine tissues and found that porcine lenses have negligible levels of sorbitol dehydrogenase and the lowest AR levels compared to other porcine tissues. ${ }^{14}$ Hence, the porcine lens is an optimal model for our study. First, the low AR levels in porcine eyes minimize newly produced sorbitol. Second, the sorbitol dehydrogenase deficiency in porcine eyes prevents the experimentally provided sorbitol from being converted into metabolites and affecting the measurement of the sorbitol concentration.

These quantitative determinations of sorbitol in the lenses were all performed via invasive biopsies, which also limits current clinical research. Because of this, there is a large need for a non-destructive and non-invasive method to measure sorbitol concentrations in human lenses. Szwergold et al. reported elevated Phosphorus-31 nuclear magnetic resonance $\left({ }^{31} \mathrm{P}\right.$ NMR) signal for D-sorbitol 3-phosphate in diabetic rat lenses. ${ }^{15}$ Lal et al. quantified sorbitol in porcine lenses ex vivo by ${ }^{31} \mathrm{P} \mathrm{NMR},{ }^{16}$ since ${ }^{31} \mathrm{P}$ has a relatively high gyromagnetic ratio, which makes it easy to be detected by NMR and is more suitable to be used as a tracer comparing to ${ }^{1} \mathrm{H}$ and ${ }^{19} \mathrm{~F}$. Hence, sorbitol needs to be labeled by Phosphorus-31 and formed as a sorbitol 3-phosphate compound. This pretreatment for labeling is time consuming for lens cultures and perfusion and limits its potential for in vivo applications. Raman spectroscopy can quantitatively determine target molecules via its vibrational or rotational mode generated by the excitation light without any pretreatment for labeling. It has been proven to be a powerful tool in ophthalmic applications ${ }^{17}$ and has been used to investigate the lens extensively. ${ }^{18,} 19$ Ergin and Thomas detected injected nearphysiological-level glucose in porcine eyes ex vivo by a compact Raman system ${ }^{20}$. Furić et al. examined diabetic rat lenses by fluorescent background index and water band index based on Raman spectrum. ${ }^{21}$ Paluszkiewicz et al. analyzed human lenses with cataract formation by Raman spectroscopy in vitro ${ }^{22}$ and later compared Raman spectra of cataractous and non-cataractous human lenses. ${ }^{23}$ Vrensen et al. analyzed protein profiles in aged human donor lenses with cortical, nuclear, and mixed cataracts. ${ }^{24}$ Huang et al. reviewed in detail Raman spectroscopy applications on the cataractous lens with different sample statuses, e.g. histologic specimens and intact animal lenses. ${ }^{19}$ However, sorbitol was 
not mentioned although it has relatively strong Raman signals ${ }^{25-27}$ that make it an optimal target composition for diabetic cataract investigation by Raman spectroscopy.

In this study, the feasibility of using Raman spectroscopy to detect sorbitol in cataractous porcine lenses is investigated. Concentration curves of sorbitol were obtained in- and outside cataractous porcine lenses and the correlation between the sorbitol concentration and cataract grade was determined.

\section{Methods}

\section{Confocal Raman spectroscopy system}

Figure 2 shows a schematic description of the confocal Raman spectroscopy (CRS) setup used in this study. Lasers with a wavelength of $671 \mathrm{~nm}$ (Laser Quantum Ignis 671, SMD 6000, Konstanz, Germany) and a 785 nm (Innovative Photonic Solutions SM 785 nm, Monmouth Junction, NJ, United States) were used as excitation light sources with a power of $14 \mathrm{~mW}$ and $26 \mathrm{~mW}$, respectively. The excitation light was coupled into a commercial high-performance Raman spectrometer module (Model 2500, River Diagnostics ${ }^{\circledR}$, Rotterdam, the Netherlands) by diamond optical fibers. A $25 \mu \mathrm{m}$ pinhole was integrated within the spectrometer to achieve a confocal approach. The output beam from the spectrometer was first collimated with an $80 \mathrm{~mm}$ focus length (f80) and then guided by a mirror to a $\mathrm{f} 60$ focus lens to reach the sample. The sample was placed on a 3D stage for fine adjustment. The generated Raman scattering light was collected using the same light path. Raman spectra were measured with an integrated CCD camera (operating temperature $\left.-60^{\circ}\right)$. Integration times of 60 seconds and 10 seconds were applied for spectra collection in the fingerprint region $\left(400 \mathrm{~cm}^{-1}\right.$ to $\left.1800 \mathrm{~cm}^{-1}\right)$ and high wavenumber region $\left(2400 \mathrm{~cm}^{-1}\right.$ to $\left.4000 \mathrm{~cm}^{-1}\right)$, respectively.

\section{Sample preparation}

Eight fresh enucleated eyes from 6 month old domestic porcine (Sus Scrofa Domesticus) were obtained from a local abattoir. The eyes were transported to the laboratory on ice and lenses were extracted immediately after arrival. The extracted lensed were washed by phosphate buffer saline (PBS) ${ }^{21}$ and submerged in PBS diluted sorbitol (\#S1876, Sigma-Aldrich, MO, United States) solutions in a 12-wells plate. The following sorbitol concentrations used: $2 \mathrm{mM}, 5 \mathrm{mM}, 10 \mathrm{mM}, 20 \mathrm{mM}, 30 \mathrm{mM}, 40 \mathrm{mM}$, and $50 \mathrm{mM}$. As a negative control, we submerged one porcine eye in PBS. The submerged eyes were stored for 24 hours in a $4^{\circ} \mathrm{C}$ environment. 


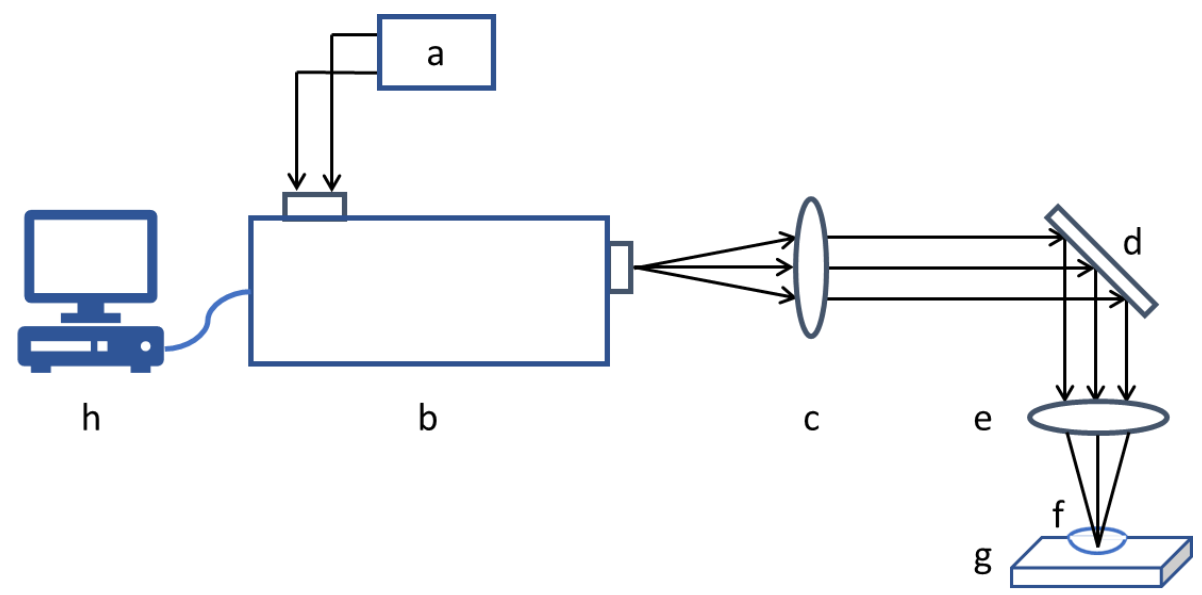

Fig. 2 Raman spectroscopy system. (a) Excitation laser with wavelength of $671 \mathrm{~nm}$ and $785 \mathrm{~nm}$; (b) Raman spectrometer with $25 \mu \mathrm{m}$ pinhole; (c) f80 collimating lens; (d) Reflecting mirror; (e) f60 focusing lens; (f) Sample (porcine lens); (g) 3D stage; (h) Computer. Lines with arrow is indicating the light path in a confocal Raman spectroscopy system.

\section{Data processing}

Raman spectra were processed by a self-developed program with Matlab (Version 2017b, The Mathworks Inc., Natick, MA, United States). ${ }^{28}{ }^{29}$ Cosmic ray removal and background correction was performed before Raman spectrum data analysis. Spectra were normalized based on the strongest peak intensity (at a wavenumber of $1004 \mathrm{~cm}^{-1}$ in fingerprint and $3388 \mathrm{~cm}^{-1}$ in high wavenumber).

Statistical analyses were conducted by SPSS (SPSS Inc., Chicago, IL, USA, version 22.0). Variables were checked for normal distribution by Shapiro-Wilk's test. Correlations between sorbitol concentration and cataract were calculated with Pearson.

\section{Results}

\section{Raman spectra of the sorbitol acquired in cuvette}

Fig. 3a shows raw Raman spectra of sorbitol solution of $0 \mathrm{mM}$ and $50 \mathrm{mM}$ without background subtraction. For statistical analyses, we used the Sorbitol peak at $876 \mathrm{~cm}^{-1} .^{26}$ The peak at $990 \mathrm{~cm}^{-1}$ is from additives in the PBS solution. ${ }^{30}$ Fig. $3 \mathrm{~b}$ shows the concentration curve from $2 \mathrm{mM}$ to $50 \mathrm{mM}$ sorbitol solution with a R-square of the linear fitting concentration of 0.997 and Pearson's R of 0.999 . 

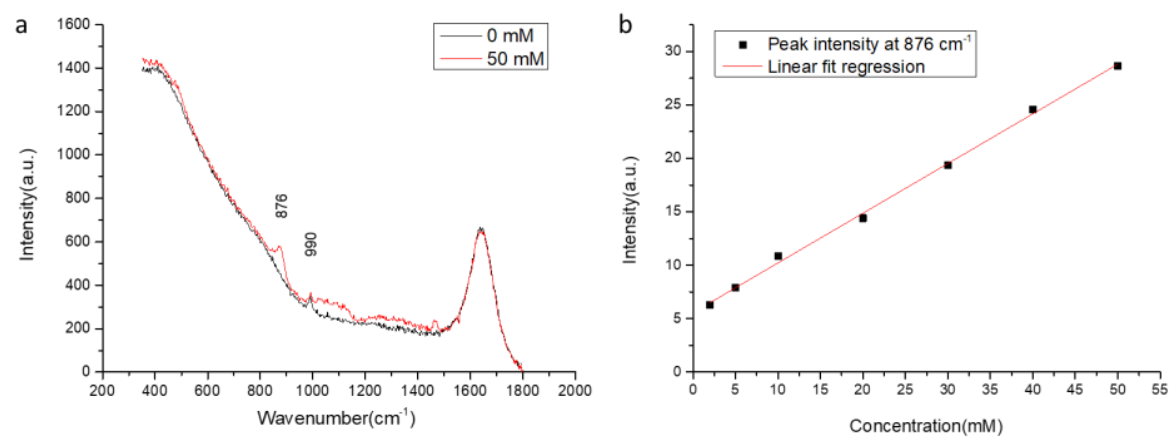

Fig. 3 Raman spectrum of sorbitol. a) Raman spectrum of control and $50 \mathrm{mM}$ sorbitol solution. b) The linear regression of the sorbitol concentration from $2 \mathrm{mM}$ to $50 \mathrm{mM}$.

\section{Raman spectrums of the untreated porcine lens acquired ex vivo}

Fig. 4 shows Raman spectra obtained from untreated porcine lenses (Fig. 4a and 4b). We used the ratio between peak $1032 \mathrm{~cm}^{-1}$ and $1004 \mathrm{~cm}^{-1}$ as a negative control. Table 1 shows the assignments of the peaks found in the porcine lens. The variance in the Raman peaks intensity in fresh porcine lenses was less than $4 \%$.
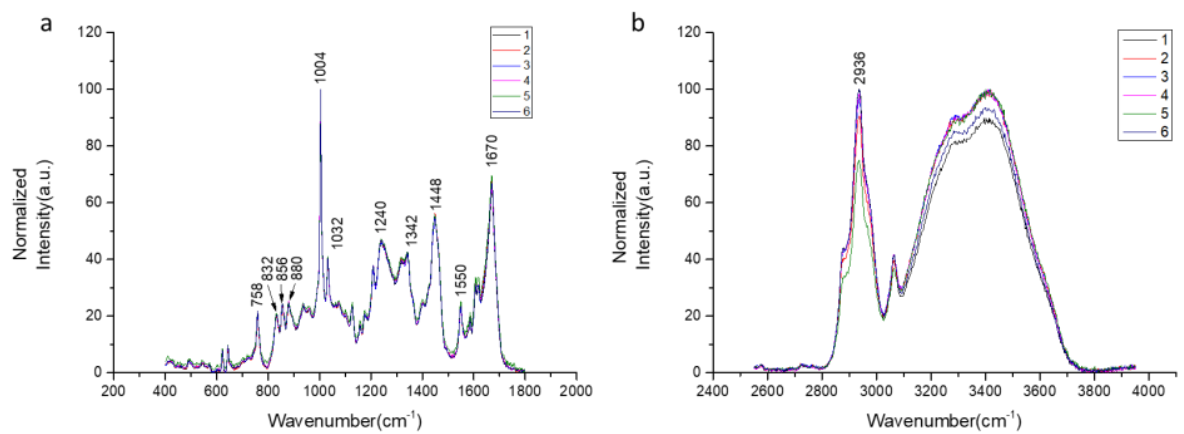

Fig. 4 Raman spectra of the fresh porcine lens. a) Fingerprint region of 5 fresh porcine lenses at a depth of $2 \mathrm{~mm}$. b) High wavenumber region of 5 fresh porcine lenses at a depth of $2 \mathrm{~mm}$. 
Table 1. The origins of Raman peaks (wavenumber in $\mathrm{cm}^{-1}$ ) in porcine lens. ${ }^{19,31-33}$

\begin{tabular}{ccc}
\hline Assignment & Wavenumber & Intensity \\
\hline Tryptophan & 758 & Medium \\
& 1342 & Weak \\
& 1550 & Medium \\
Tyrosine & 832 & Weak \\
\hline \multirow{2}{*}{ Phenylalanine } & 856 & Weak \\
& 1003 & Weak \\
\hline Amide III & 1032 & Strong \\
& 1240 & Medium \\
\hline Amide I & 1448 & Medium \\
\hline Aliphatic residues & 1670 & Medium \\
\hline
\end{tabular}

Raman spectrums of the sorbitol porcine lens acquired ex vivo

Raman spectra from treated porcine lenses were acquired to identify the sorbitol substance and evaluate cataracts. The ratio of the intensities of the sorbitol peak $\left(876 \mathrm{~cm}^{-1}\right)$ and the phenylaniline peak at $1004 \mathrm{~cm}^{-1}$ is used to show the sorbitol concentration differences. For the cataract grades, we used the ratio between the intensities of the protein peak at $2934 \mathrm{~cm}^{-1}$ and the water peak at $3388 \mathrm{~cm}^{-1}$. Estimated sorbitol concentrations are plotted in Fig. 5a and cataract grades are plotted in Fig. 5b. They show to be linear with the tested concentrations with R-square of 0.874 and Pearson's R of 0.946 , the grades of the cataract with R-square of 0.792 and Pearson's R of 0.907 , respectively. 

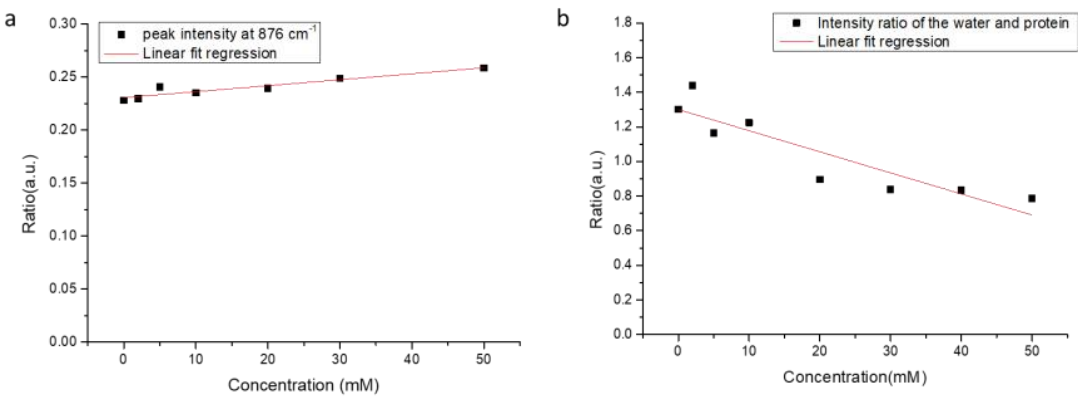

Fig.5 Linear regression analysis of the Raman spectroscopy of sorbitol concentration and cataract grades. a) Sorbitol concentration ratio measured from 0mM (PBS only) to $50 \mathrm{mM}$ treated lens; $\mathrm{b}$ ) The linear regression of the cataract grades by the ratio of Raman peaks intensity.

\section{Statistical analyses results}

We found a Pearson correlation of 0.946 between actual concentration and estimated sorbitol concentration is $(p=0.01)$. The Pearson correlation between estimated sorbitol concentration and the cataract grade was $0.823(p=0.023)$.

\section{Discussion}

In this study, sorbitol was detected by confocal Raman spectroscopy down to a clinical relevance concentration of $2 \mathrm{mM}$ in mimicked cataractous porcine lenses ex vivo. In the sorbitol solution, the concentration curve shows great linearity with an R-square of 0.997 and Pearson's R of 0.999. The Raman spectra are in line with Mignani et al. that showed the peak at $876 \mathrm{~cm}^{-1}$ can be used for sorbitol identification. ${ }^{26}$ The concentration curve indicates that Raman intensities at the peak $876 \mathrm{~cm}^{-1}$ have a good linear response along with the sorbitol concentration in its chemical form.

Cataract grades were quantitatively evaluated using Raman peak intensity ratios and showed a significant correlation with sorbitol concentrations. Raman spectra from fresh porcine lenses (Fig.4) were used to identify the background peaks in the lens. The lens consists mainly of proteins together with saturated fatty acids, cholesterol, sphingomyelin, and water distributed in different structures. The Raman spectrum of a fresh porcine lens was similar to those found by Medina-Gutiérrez et al. who investigated the amino acid contents in porcine lenses using Raman spectroscopy. ${ }^{31}$ We also noticed that all major peak origins from proteins in porcine lenses listed in Table 1 can also be observed in the aged human lenses with cataracts. ${ }^{24}$ This indicates that only using protein peaks might lack specificity in distinguishing cataract lenses caused by different reasons. In addition, due to background interference (e.g., fluorescence), data processing of Raman spectroscopy is challenging. Since there is no well-accepted standardized procedure for data processing in bio-spectroscopy yet ${ }^{34}$ the processed results may lead to a biased conclusion. 
The fluorescence background of the spectrum and water spectra both showed a positive correlation with the cataract grades on a rat model. ${ }^{21}$ Similarly, several researchers reported using the peak intensity ratio method to quantitatively evaluate the water content in cataractous lenses. ${ }^{19,24}$ However, this water ratio method is not only used in diabetic cataract studies but also used in hereditary cataracts and trauma-induced cataracts studies. ${ }^{35}$, 36 Therefore, only using water/protein ratios is inadequate to specifically study diabetic cataracts. In complement, biomarkers like glucose are proposed by previous studies for diabetes mellitus investigations. Lambert et al. and Borchert et al. measured the glucose concentration in the aqueous humor in animal models. ${ }^{37,38}$ Pelletier et al. further measured the glucose concentrations in aqueous humor samples obtained from patients undergoing cataract surgery. ${ }^{39}$ However, the detection limit of Raman spectroscopy on glucose was below its concentration differences in a molecule profile analysis. ${ }^{10}$ In this study, no concentration difference of glucose between the diabetic cataract lenses and health lenses was reported. On the contrary, a 20 fold increase of sorbitol concentration in diabetic cataract lenses was reported in the same study. We, therefore, proposed to measure sorbitol for diabetic cataract lens. Acquiring sorbitol concentration levels might also give more insight into the osmotic stress hypothesis of cataracts. The outcome of our Raman measurements shows that the detection limit of sorbitol can reach the accumulated concentration in diabetic cataract lenses and further shows sorbitol is correlated with the grades of the cataract.

Raman intensities might be influenced by several issues. First, the lens encompasses different structures, such as the epithelium, cortex and nucleus. Data obtained in different locations of the lens might provide different results. Hence, lens orientation was taken into account. In addition, sorbitol formation associated with AR activity occurs in the epithelium and superficial cortical fibers ${ }^{40}$ and AR is implicated in lens epithelium-tomesenchymal transition ${ }^{41}$. The laser focus is therefore positioned at a depth of $2 \mathrm{~mm}$, which is under the epithelium and among superficial cortical fibers. Second, research has indicated that a low storage temperature of the lens might increases the sorbitol extracellular space. In rat lenses, sorbitol increased when the incubation temperature was decreased from $37^{\circ} \mathrm{C}$ to $14^{\circ} \mathrm{C} .{ }^{42}$ Similar elevation has been found in clear human lenses obtained from eye bank eyes. ${ }^{43}$ The temperature dynamics between storage and measurement in this study might contribute to the intensity variance and disturb the linearity of the sorbitol concentration regression in the lenses. Furthermore, Raman intensity also can be affected by the opacity of the cataractous lens or inhomogeneity of the tissue. Hence, the R-square of the sorbitol concentration linear regression obtained in the lens (0.874) is lower than that of its chemical form (0.997). In addition, the status of the porcine lens is also crucial to calculate the intensity ratios of water content when classifying the grades of cataract. Therefore, the lenses were kept moisture to avoid extra dehydration. Nonetheless, the R-square of the cataract grades linear regression is only 0.792. The ratio is calculated by using the protein peak to divide the water peak. It was 
reported that the absolute water content varies from the anterior to the posterior regions along with depth in rabbit lenses. ${ }^{44}$ Besides, Vrensen et al. showed that the Raman spectrum of cataractous lenses shares common peaks of protein at the nucleus, deep cortex and superficial cortex with only slight differences at the intensities. ${ }^{24}$ Since we positioned at the same depth in each lens, the absolute water content barely changes and the difference from protein at variant locations can be neglected. Thus, the decrease in the ratio (Fig. 5b) reflects the increment in the water content in cataractous lenses, in agreement with previously reported research. ${ }^{19,21,24,44}$ Furthermore, the presented water content ratio of 1.44/0.78 (highest versus lowest value in Fig. 5b) in lens supports Horikiri et al. results, which claimed an approximately 2-3 times increment of water content in a cataractous lens. ${ }^{35}$ In the present study, the Pearson correlation between the sorbitol concentration and the grades of diabetic cataractous lenses was $0.823(p=0.023)$ indicating sorbitol measurements in diabetic cataract lens may reveal valuable information supporting the osmotic hypothesis and be used as an indicator in identifying diabetic cataracts.

For proof of concept, we only collected the spectrum from one location (at a depth of $2 \mathrm{~mm}$ ) while cataracts might also occur at the anterior polar, cortical, subcapsular, and posterior subcapsular. ${ }^{7}$ Multiple location investigations of the lens could provide beneficial for understanding how diabetes causes cataract development. In addition, to mimic a cataractous lens we immersed a lens in sorbitol solutions. Alternative treatment to introduce cataract in lenses are optional; Alvarez-Rivera et al. use M-199 medium with L-glutamine, sodium bicarbonate and antibiotics for in vitro anti-cataract study. ${ }^{45}$ However, this treatment will lead to a significant increase in the background of the Raman spectrum due to additives. More measurements will be planned with diabetic cataract induced animal models and human subjects to gain more insights into the mechanisms of diabetic cataract development.

\section{Conclusion}

Raman spectra of sorbitol obtained from mimicked cataractous porcine lenses show a significant correlation with the grades of cataract. We feel that the detection limit of the sorbitol concentration in lenses may be clinically relevant in distinguishing diabetic cataracts from cataracts caused by other reasons in the early stages. Raman spectroscopy may prove not only to be a substantial non-invasive and non-labeling diagnostic tool for diabetic cataracts, but also a tool for investigating and follow up on the formation of cataracts in general.

\section{Funding}

Shuo Zhang and Qi Qiao acknowledge the China Scholarship Council for the support by State Scholarship Fund No. 201309110103 and No.201707720057, respectively. 


\section{Conflicts of interest}

The authors declare no conflict of interest.

\section{Acknowledgements}

The authors thank Jackson T. Boonstra for English editing. 


\section{Reference:}

1. H. Kiziltoprak et al., "Cataract in diabetes mellitus," World J Diabetes 10(3), 140-153 (2019).

2. I. G. Obrosova, S. S. Chung, and P. F. Kador, "Diabetic cataracts: mechanisms and management," Diabetes Metab Res Rev 26(3), 172-180 (2010).

3. R. Klein, and B. E. Klein, "Vision disorders in diabetes," Diabetes in America 1(293 (1995).

4. M. Brownlee, "The pathobiology of diabetic complications - A unifying mechanism," Diabetes 54(6), 1615-1625 (2005).

5. A. S. Grewal et al., "Updates on Aldose Reductase Inhibitors for Management of Diabetic Complications and Non-diabetic Diseases," Mini Rev Med Chem 16(2), 120-162 (2016).

6. J. H. Kinoshita, "pathway of glucose metabolism in the lens," Investigative Ophthalmology \& Visual Science 4(10 (1965).

7. A. Pollreisz, and U. Schmidt-Erfurth, "Diabetic cataract-pathogenesis, epidemiology and treatment," $J$ Ophthalmol 2010(608751 (2010).

8. M. Lorenzi, "The Polyol Pathway as a Mechanism for Diabetic Retinopathy: Attractive, Elusive, and Resilient," Exp Diabetes Res (2007).

9. M. Lorenzi, "The polyol pathway as a mechanism for diabetic retinopathy: attractive, elusive, and resilient," Exp Diabetes Res 2007(61038 (2007).

10. S. Yoshioka et al., "Effect of diabetes on the free polyol pattern in cataractous lenses," Clin Chem 37(5), 686-689 (1991).

11. J. F. KUCK, "Carbohydrates of the lens in normal and precataractous states," Investigative Ophthalmology \& Visual Science 4(4), 638-642 (1965).

12. J. A. Jedziniak et al., "The sorbitol pathway in the human lens: aldose reductase and polyol dehydrogenase," Investigative ophthalmology \& visual science 20(3), 314-326 (1981).

13. J. I. Malone, S. Lowitt, and W. R. Cook, "Nonosmotic diabetic cataracts," Pediatric research 27(3), 293-296 (1990).

14. G. Vaca et al., "Sorbitol dehydrogenase deficiency in several pig tissues: potential implications for studies of experimental diabetes," Diabetologia 27(4), 482-483 (1984).

15. B. S. Szwergold et al., "Identification of D-Sorbitol 3-Phosphate in the Normal and Diabetic Mammalian Lens," Journal of Biological Chemistry 264(16), 9278-9282 (1989).

16. S. Lal et al., "Detection of fructose-3-phosphokinase activity in intact mammalian lenses by 31P NMR spectroscopy," Journal of Biological Chemistry 268(11), 7763-7767 (1993).

17. R. J. Erckens et al., "Raman spectroscopy in ophthalmology: from experimental tool to applications in vivo," Lasers Med. Sci. 16(4), 236-252 (2001).

18. C.-C. Lin, "Review: Raman Spectroscopy - A Novel Tool for Noninvasive Analysis of Ocular Surface Fluid," Journal of Medical and Biological Engineering 30(6), 343 (2010).

19. C.-C. Huang, and W. Chen, "Raman spectroscopic analysis of cataract lens: A compendious review," Applied Spectroscopy Reviews 53(9), 689-702 (2018).

20. A. Ergin, and G. Thomas, "Noninvasive detection of glucose in porcine eyes," Proceedings of the IEEE 31st Annual Northeast Bioengineering Conference, 2005. 246-247 (2005).

21. K. Furić, V. Mohaček-Grošev, and M. Hadžija, "Development of cataract caused by diabetes mellitus: Raman study," Journal of Molecular Structure 744-747(169-177 (2005).

22. C. Paluszkiewicz et al., "Analysis of human lenses by Raman microspectroscopy," Acta Physica Polonica A 129(2), 244-246 (2016).

23. C. Paluszkiewicz et al., "Vibrational microspectroscopy analysis of human lenses," Spectrochim Acta A Mol Biomol Spectrosc 188(332-337 (2018).

24. G. Vrensen et al., "Protein profiles in cortical and nuclear regions of aged human donor lenses: A confocal Raman microspectroscopic and imaging study," Exp Eye Res 145(100-109 (2016).

25. C. Camerlingo et al., "Micro-Raman spectroscopy and univariate analysis for monitoring disease follow-up," Sensors (Basel) 11(9), 8309-8322 (2011).

26. A. G. Mignani et al., "Raman Spectroscopy for Distinguishing the Composition of Table-top Artificial Sweeteners," Procedia Engineering 87(240-243 (2014).

27. M. de Veij et al., "Reference database of Raman spectra of pharmaceutical excipients," Journal of Raman Spectroscopy 40(3), 297-307 (2009).

28. C. J. F. Bertens et al., "Confocal Raman spectroscopy: Evaluation of a non-invasive technique for the detection of topically applied ketorolac tromethamine in vitro and in vivo," Int. J. Pharm. 570(118641 (2019).

29. C. J. F. Bertens et al., "Pipeline for the removal of hardware related artifacts and background noise for 
Raman spectroscopy," MethodsX 7(100883 (2020).

30. C. David et al., "Raman and IR spectroscopy of manganese superoxide dismutase, a pathology biomarker," Vibrational Spectroscopy 62(50-58 (2012).

31. C. Medina-Gutiérrez et al., "Amino acid contents along the visual and equatorial axes of a pig lens by Raman spectroscopy," Spectrochimica Acta Part A: Molecular and Biomolecular Spectroscopy 60(10), 2269-2274 (2004).

32. C. Medina-Gutierrez et al., "Study of the protein distribution in the pig lens cross section by Raman spectroscopy," Spectrochim Acta A Mol Biomol Spectrosc 60(7), 1573-1577 (2004).

33. I. Siebinga et al., "Ageing and changes in protein conformation in the human lens: a Raman microspectroscopic study," Exp Eye Res 54(5), 759-767 (1992).

34. H. J. Byrne et al., "Spectral pre and post processing for infrared and Raman spectroscopy of biological tissues and cells," Chem Soc Rev 45(7), 1865-1878 (2016).

35. K. Horikiri et al., "Estimation of structural changes in the cataractous rat lens using Raman spectroscopy," Experimental Animals 41(2), 225-230 (1992).

36. K. Nakamura et al., "1H-NMR and Raman studies on perforating trauma-induced cataract formation in a mouse lens," Biochimica et Biophysica Acta (BBA) - General Subjects 1474(1), 23-30 (2000).

37. J. Lambert, M. Storrie-Lombardi, and M. Borchert, "Measurement of physiologic glucose levels using Raman spectroscopy in a rabbit aqueous humor model," LEOS Newsletter 4(19), 5 (1998).

38. M. S. Borchert, M. C. Storrie-Lombardi, and J. L. Lambert, "A noninvasive glucose monitor: preliminary results in rabbits," Diabetes Technol Ther 1(2), 145-151 (1999).

39. C. C. Pelletier, J. L. Lambert, and M. Borchert, "Determination of glucose in human aqueous humor using Raman spectroscopy and designed-solution calibration," Appl. Spectrosc. 59(8), 1024-1031 (2005).

40. $\quad$ A. J. Bron et al., "The lens in diabetes," Eye 7(2), 260-275 (1993).

41. G. J. Zablocki et al., "Aldose reductase-mediated induction of epithelium-to-mesenchymal transition (EMT) in lens," Chem Biol Interact 191(1-3), 351-356 (2011).

42. J. W. Patterson, "A review of glucose transport in the lens," Investigative Ophthalmology \& Visual Science 4(4), 667-679 (1965).

43. L. T. Chylack et al., "Efficacy of Alrestatin, An Aldose Reductase Inhibitor, in Human Diabetic and Nondiabetic Lenses," Ophthalmology 86(9), 1579-1585 (1979).

44. A. Huizinga et al., "Local variation in absolute water content of human and rabbit eye lenses measured by Raman microspectroscopy," Exp Eye Res 48(4), 487-496 (1989).

45. F. Alvarez-Rivera, A. Concheiro, and C. Alvarez-Lorenzo, "Epalrestat-loaded silicone hydrogels as contact lenses to address diabetic-eye complications," Eur J Pharm Biopharm 122(126-136 (2018). 


\section{Chapter 4}

Confocal Raman spectroscopy: Evaluation of a noninvasive technique for the detection of topically applied ketorolac tromethamine in vitro and in vivo

Christian J.F. Bertens", Shuo Zhang ${ }^{\#}$, Roel J. Erckens, Frank J.H.M. van den Biggelaar, Tos T.J.M. Berendschot, Carroll A.B. Webers, Rudy M.M.A. Nuijts and Marlies Gijs \# Equal contributing International Journal of Pharmaceutics 2019;570:118641 DOI: 10.1016/j.ijpharm.2019.118641 


\begin{abstract}
Current information about the pharmacokinetics of an ocular drug can only be achieved by invasive sampling. However, confocal Raman spectroscopy bears the potential to quantify drug concentrations non-invasively. In this project, we evaluated the detection and quantification of ocular ketorolac tromethamine levels with confocal Raman spectroscopy after topical administration.

Confocal Raman spectroscopy and high-performance liquid chromatography (HPLC) were compared in terms of sensitivity of detection. Enucleated pig eyes were treated with different concentrations of ketorolac. Hereafter, ketorolac concentrations in the aqueous humor of pig eyes were analyzed by confocal Raman spectroscopy and HPLC.

Subsequently, twelve rabbits were treated with Acular ${ }^{\mathrm{TM}}$ for four weeks. At several time points, ketorolac concentrations in aqueous humor of the rabbits were measured by confocal Raman spectroscopy followed by drawing an aqueous humor sample for HPLC analysis.

In ketorolac treated pig eyes, both ex vivo Raman spectroscopy as well as HPLC were able to detect ketorolac in a broad concentration range. However, in vivo confocal Raman spectroscopy in rabbits was unable to detect ketorolac in contrast to HPLC.

To conclude, confocal Raman spectroscopy has the capacity to detect ketorolac tromethamine in vitro, but currently lacks sensitivity for in vivo detection.
\end{abstract}

\title{
Graphical abstract
}

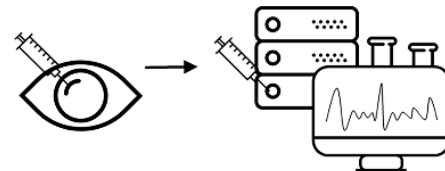

Invasive sampling, purification and HPLC analysis

VS.

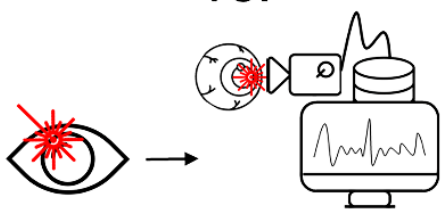

In vivo, non-invasive and realtime Raman detection
In vitro
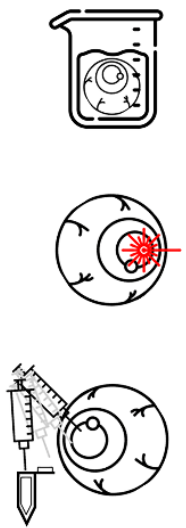

In vivo

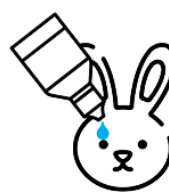

Drug treatment

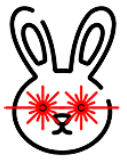

Raman

spectroscopy

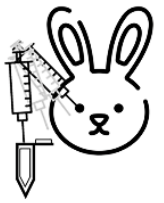

Cuvette analysis

- Raman spectroscopy

- HPLC 


\section{Introduction}

Ocular pharmacokinetic studies investigate time- and dose dependent behavior of ophthalmic drugs. These studies are important to detect the maximum drug concentration $\left(\mathrm{C}_{\max }\right)$, the time to reach $\mathrm{C}_{\max }\left(\mathrm{T}_{\max }\right)$, half-life, and clearance of the drug. Based on those parameters, a dosage regimen can be created. ${ }^{1}$ Evaluation of a pharmacokinetic profile should include assessment of systemic exposure (i.e., blood, plasma or serum levels) as well the distribution and levels in ocular tissues (e.g., cornea, iris, aqueous humor). Currently, the assessment of ocular pharmacokinetics is using tissues or fluids in a destructive test which comprises chemical pre-treatment followed by high-performance liquid chromatography (HPLC). ${ }^{2}$ Besides extensive processing time, also sampling has been a challenge as ocular tissues or fluids cannot be harvested without interfering with the anatomical integrity of the eye (e.g., during intraocular surgery). Due to the limited accessibility of samples from humans, and the destructiveness of the method, pharmacokinetic research is relying on large quantities of animals, e.g. rabbits, dogs, pigs, and monkeys, ${ }^{3}$ because the eyes of these animals show similarities to human eyes. ${ }^{4}$ Therefore, animal experiments have been widely criticized for both, ethical and economical reasons. ${ }^{5}$

A non-invasive pharmacokinetic assessment technique could resolve these issues. A technique that is potentially suitable for non-invasive detection of ocular pharmacokinetics is Raman spectroscopy. Raman spectroscopy identifies molecules, based on the specific inelastic scattering properties of their rotational and vibrational modes. ${ }^{6-8}$ This technique enables real-time detection of molecules without pre-processing and damaging tissue. As such, the number of animals and its associated costs needed for ocular pharmacokinetic studies can be reduced. Compared to infrared (IR) spectroscopy, Raman spectroscopy bears the advantage that molecules do not have to possess a permanent dipole moment, therefore, more molecules can be detected. Furthermore, Raman spectroscopy is not affected by aqueous samples, whereas IR is absorbed intensively by water. Near-infrared (NIR) spectroscopy (NIRS) is, like Raman spectroscopy, not affected by aqueous samples. However, absorption bands tend to overlap in NIRS, which results in less accessible molecule-specific information, thus a lower specificity compared to Raman spectroscopy. ${ }^{9}$

Since Raman spectroscopy is a scattering technique, fiber-optics and remote sampling can be used. Samples can be measured directly in glass container or in case of pharmaceuticals, samples can be measured in original sachets. Because of a high spatial resolution, components from complex samples can be identified (e.g., cell-media components from a commercial recombinant-protein manufacturing process).$^{10}$ However, due to the weak nature of Raman scattering $\left(1\right.$ in $10^{9}$ or $\left.10^{10}\right),{ }^{11}$ higher sensitivity of Raman spectroscopy requires higher power lasers as excitation source. Therefore, Raman spectroscopy is very suitable for chemical applications; but the photo-thermal effects in light-tissue interaction could cause concerns in vivo if the wrong wavelength is selected as emitting source. If the local temperature reaches up to $43.0^{\circ} \mathrm{C}$ hyperthermia will occur. A 
temperature up to $70^{\circ} \mathrm{C}$ could lead to tissue coagulation and welding. Higher temperatures (above $100^{\circ} \mathrm{C}$ ) cause vaporization and (above $300^{\circ} \mathrm{C}-450^{\circ} \mathrm{C}$ ) carbonization. ${ }^{12}$ Another challenge for Raman spectroscopy is interference from fluorescence. Biological samples often emit fluorescence signals in the same wavelength range as Raman signals. ${ }^{2}$ Finally, data processing of Raman spectroscopy is a challenging task. Since there is no wellaccepted standard procedure in data processing for bio-spectroscopy yet, ${ }^{13}$ each (animal) model needs a specific calibration model. In this model it is important to remove interferences such as background from the substrate. Several technical approaches have been developed to meet these challenges for the biological applications of Raman spectroscopy. ${ }^{13,14}$

As shown by our group, many ophthalmic drugs have very specific Raman fingerprint patterns (patterns specific for a drug-molecule). ${ }^{15}$ Bauer et al. demonstrated that confocal Raman spectroscopy can be used for pharmacokinetic detection of Dorzolamide ophthalmic solution in tear-film, and corneas of living rabbits. ${ }^{16}$ Another study showed the detection of glucose levels in aqueous humor in rabbits ${ }^{17}$ and human samples. ${ }^{18}$ Sideroudi et al. showed that Raman spectroscopy is of interest to test drug concentrations in an artificial anterior chamber model using ciprofloxacin as target drug. ${ }^{19}$ Ganciclovir, ceftazidime and amphotericin B have been detected with Raman spectroscopy in vitro after injecting the drugs into the anterior chamber of rabbit eyes. ${ }^{13,20}$ Although these studies demonstrate the potential of Raman spectroscopy, (animal) models are often not representative of the clinical situation. For example, the injection of drugs in the anterior chamber results in far too high drug concentrations in the aqueous humor and a limited distribution through the tissues. $^{3}$

In this study, we designed and performed in vitro and in vivo animal experiments to detect ketorolac tromethamine, a non-steroidal anti-inflammatory drug (NSAID), using Raman spectroscopy and confirm our findings by high-performance liquid chromatography (HPLC).

\section{Materials and methods}

\section{Materials}

Ketorolac trometamine was purchased from MSN laboratories (Telangana, India), ketorolac $0.5 \%$ ophthalmic solution (Acular ${ }^{\mathrm{TM}}$ ) was purchased from Allergan (Dublin, Ireland), Methoce ${ }^{\circledR} 2 \%$ was purchased from OmniVision (Santa Clara, CA, United States), and sterile buffered saline solution (BSS), $0.9 \% \mathrm{NaCl}$ solution with a of $\mathrm{pH} 7.4$ was purchased from B. Braun (Melsungen AG, Germany). Ethylenediaminetetraacetic acid (EDTA) (\#E5134), benzalkonium chloride (BAK) (\#B-1383), methanol (\#34860), and Brand $^{\circledR}$ cuvettes (\#7592-00) were purchased from Sigma-Aldrich (MO, United States). Ketamine was provided by Alfasan (Woerden, the Netherlands), midazolam by Actavis (Dublin, Ireland), and MINIMS ${ }^{\circledR}$ Oxybuprocaine hydrochloride by Bausch \& Lomb Pharma (Brussels, Belgium). Insulin syringes (BD Micro-Fine ${ }^{\mathrm{TM}}$ ) were bought from Becton 
Dickinson (NJ, United states). MilliQ water and phosphate buffered saline (PBS) (pH of 7.4) were freshly produced. Freshly enucleated eyes were kindly provided by "Slachthuis Kerkrade Holding", (Kerkrade, the Netherlands).

\section{Sample preparation}

Freshly enucleated eyes from the domestic pig (Sus Scrofa Domesticus) were obtained from an abattoir and transported to the laboratory on ice. Before use, the pig eyes were inspected using a stereo microscope (Olympus SZX9, Tokyo, Japan). Only eyes with clear corneas without visible corneal damage were used in the experiment. After removal of excess surrounding tissue, the eyes were washed in PBS. Within 3 hours after enucleation, the pig eyes were submerged in $15 \mathrm{~mL}$ of a dilution of ketorolac tromethamine in PBS in a $50 \mathrm{~mL}$ centrifugal tube. The following ketorolac concentrations were used to submerge the pig eyes: $0.05 \%, 0.1 \%, 0.125 \%, 0.25 \%, 0.5 \%, 1.0 \%, 1.25 \%, 2.5 \%$, and $5.0 \%$. As negative control, PBS was used, and as positive control Acular ${ }^{\mathrm{TM}}$ was used. Three eyes were used per concentration. The pig eyes were stored for about 24 hours in the dark at $4^{\circ} \mathrm{C}$ and were measured by Raman spectroscopy. After the Raman measurement, $100 \mu \mathrm{L}$ to $150 \mu \mathrm{L}$ aqueous humor was collected using an insulin syringe and the ketorolac concentration was investigated using Raman spectroscopy and HPLC.

\section{Raman spectroscopy set-up}

Figure 1 shows a schematic overview of the used modular confocal Raman spectroscopy system. A diode emitting laser of $785 \mathrm{~nm}$ with a continue power of $26 \mathrm{~mW}$ (Innovative Photonic Solutions SM 785 nm, Monmouth Junction, NJ, United States) and a $671 \mathrm{~nm}$ diode emitting laser with a continue power of $14 \mathrm{~mW}$ (Laser Quantum Ignis 671 and SMD 6000, Konstanz, Germany) were used to excite the samples. Raman spectra were recorded with a high-performance Raman module model 2500 (River Diagnostics ${ }^{\circledR}$, Rotterdam, the Netherlands). This module guides the laser light through a diamond optical fiber, shapes and conditions the beam through a pinhole to the measurement stage. First, the light is sent through a collimation lens with a focus length of $80 \mathrm{~mm}$ (f80). In front of the sample, a f80 lens was used when the sample was measured in a cuvette. For the ex vivo pig eye experiment, either a long-working-distance microscope objective lens (Jena lens, magnification $\times 25$; numerical aperture $=0.50$; focal length $=10 \mathrm{~mm}$; Carl Zeiss, Jena, Germany), or a lens with a focus length of $60 \mathrm{~mm}$ (f60) in combination with a Gonio lens (Haag-Streit Meridian; CGA1, Köniz, Switzerland) were used. To connect the Gonio lens to the cornea, topically applied Methocel ${ }^{\circledR} 2 \%$ was used. For the in vivo rabbit experiment the f60 lens in combination with a Gonio lens was used. The lens was also connected to the cornea using Methocel ${ }^{\circledR} 2 \%$. 


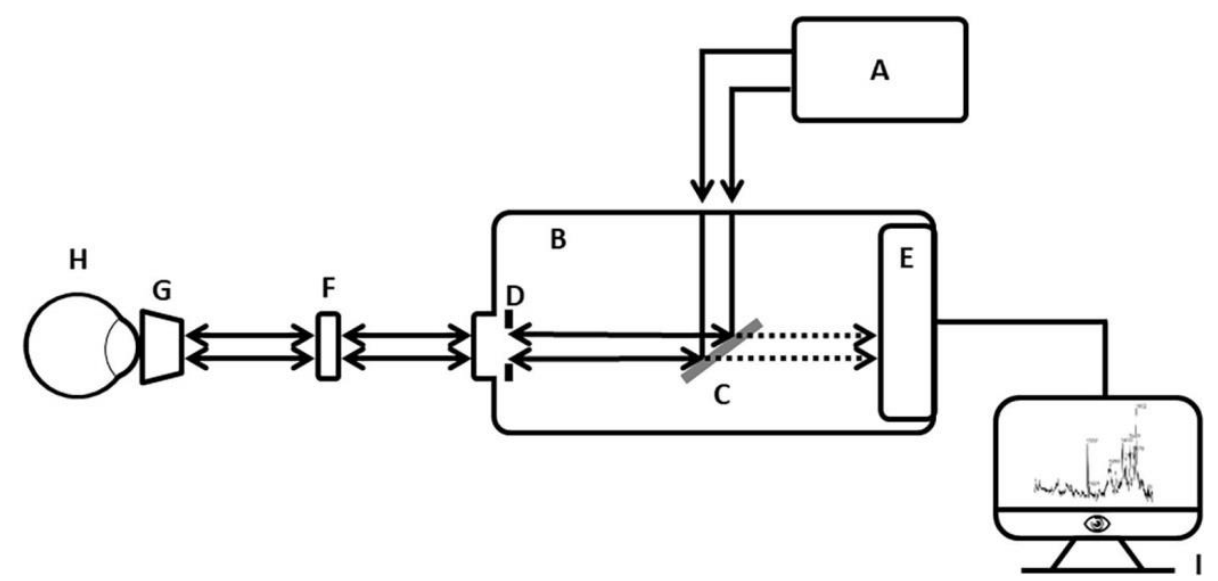

Figure 1. Schematic Raman spectroscopy set-up. (A) laser; (B) Raman module, with (C) filter for Raman scattered light; (D) $25 \mu \mathrm{m}$ pinhole; (E) integrated charge-coupled device (CCD); (F) collimation f80 lens; (G) f60 lens with a Gonio (one-mirror) lens, or objective (Jena lens), or a f80 lens; (H) sample; and (I) computer. Arrows indicate direction of (backscattered) laser light; dotted arrows indicate direction of RamanScattered light. The Raman spectrometer operates in reflectance mode.

In the experiment the lenses both act to focus the incident light as well as to collect the Raman back-scattered light. As such, the latter is passed back toward the Raman module and projected on a cooled charge-coupled device (CCD) camera (operating temperature $60^{\circ} \mathrm{C}$ ) for signal detection. Raman back-scattered light in the range of 400 relative wavenumbers $\left(\mathrm{cm}^{-1}\right)$ to $1800 \mathrm{~cm}^{-1}$ was detected using the $785 \mathrm{~nm}$ laser. For the range from $2400 \mathrm{~cm}^{-1}$ to $4000 \mathrm{~cm}^{-1}$ the $671 \mathrm{~nm}$ laser was used. The spectral resolution of the measurements was $2 \mathrm{~cm}^{-1}$ and the samples were exposed to 3 frames counting 60 seconds of exposure during the experiment. The system was used in single point modus (not confocal) and location in the sample was determined using the high wave numbers $(671 \mathrm{~nm}$ laser). ${ }^{21}$ During the in vivo rabbit experiment, the exposure was 2 frames of 30 seconds.

\section{Identification of ketorolac tromethamine}

Multivariate-peak data analysis takes into account all peaks corresponding to the chemical form ketorolac tromethamine. Elshout et al. published the four most intense peaks corresponding to ketorolac tromethamine $(1002 \mathrm{~cm}-1,1524 \mathrm{~cm}-1,1568 \mathrm{~cm}-1$ and $1602 \mathrm{~cm}-$ 1). ${ }^{15}$ In our set-up, commercially available ketorolac tromethamine eye drops (Acular ${ }^{\mathrm{TM}}$ ) were compared to each individual ingredient to investigate if the additives in Acular ${ }^{\mathrm{TM}}$ interfere with the ketorolac signal in the Raman measurement. Those ingredients were: ketorolac tromethamine (dissolved in PBS, $\mathrm{pH} 7.4)$, EDTA $(50 \mathrm{mg} / \mathrm{mL}$ dissolved in MilliQ water), BAK ( $50 \mathrm{mg} / \mathrm{mL}$ dissolved in MilliQ water). The anesthetics used in the in vivo experiment were also individually measured using Raman spectroscopy, those compounds were: ketamine, midazolam and Oxybuprocaine hydrochloride. $75 \mu \mathrm{L}$ of each sample was pipetted in a Brand ${ }^{\circledR}$ cuvette and measured using Raman spectroscopy. 


\section{Detection of ketorolac tromethamine in aqueous humor of pig eyes}

An anterior chamber paracentesis of pig eyes was done to collect $100 \mu \mathrm{L}$ to $150 \mu \mathrm{L}$ aqueous humor. After centrifugation $\left(15,000 \mathrm{G}, 5\right.$ minutes at $4{ }^{\circ} \mathrm{C}$ to remove proteins) the supernatant was transferred to a cuvette and measured with Raman spectroscopy.

Hereafter, the samples were fivefold diluted using methanol, centrifuged once more $\left(15,000 \mathrm{G}\right.$ for 5 minutes at $\left.4^{\circ} \mathrm{C}\right)$ where after the supernatant was analyzed by HPLC (Agilent 1260 infinity series with EZchrom software, Agilent inc. Santa Clara, CA, United States). Analysis was done according to the US Pharmacopeia ${ }^{22}$, using an elution time of 20 minutes and injection volume of $10 \mu \mathrm{L}$, and peak UV-detection at $313 \mathrm{~nm}$ on a symmetry

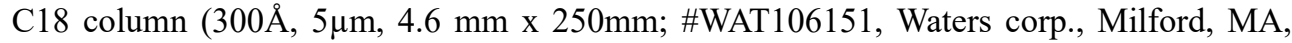

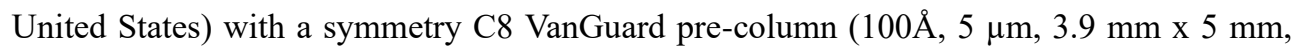
3/pkg, \#186007739, Waters corp., Milford, MA, United States). Ketorolac had a retention time of 10.5 minutes, has a limit of detection (LOD) of $4 \mathrm{ng} / \mathrm{mL}$, and a limit of quantification (LOQ) of $10 \mathrm{ng} / \mathrm{mL}$.

\section{In vivo detection of ketorolac tromethamine in rabbits}

All animal procedures were conducted according to the ARVO Statement for the Use of Animals in Ophthalmic and Visual Research and the Guidelines of the Central Laboratory Animal Facility of Maastricht University. All protocols were approved by the Central Committee for Animal research and were in accordance with the European Guidelines (2010/63/EU).

Twelve New-Zealand white rabbits (weight between $2.0 \mathrm{~kg}$ and $2.5 \mathrm{~kg}$ upon arrival) were ordered from Envigo (Horst, NL) and housed in group housing, 6 animals per cage, males and females separated. The rabbits had ad libitum access to water and received 100 gr. rabbit chow per animal per day. Before the animals were used in experiment, they had one week to acclimatize. The rabbits were treated with $50 \mu \mathrm{L} 0.5 \%$ ketorolac tromethamine ophthalmic solution (Acular ${ }^{\mathrm{TM}}$ ) in the lower conjunctival fornix. The contralateral eye was treated with $50 \mu \mathrm{L}$ BSS. Both treatments were performed three times a day for a total of 28 days, equivalent to a clinically used drop regime.

On day 0 , day 7 , day 14 , day 21 , and day 28 the rabbits were measured. All measurements were performed 1 to 3 hours after receiving the eye drops. During the examinations, rabbits were anesthetized with ketamine $(50 \mathrm{mg} / \mathrm{kg})$ and midazolam (5 $\mathrm{mg} / \mathrm{kg}$ ) intramuscularly. First, a Raman measurement was performed on both eyes, followed by an anterior chamber paracentesis (drawing $50 \mu \mathrm{L}$ ) of the right eye. Before the paracentesis, the eye received additional topical sedation using one drop of $0.4 \%$ Oxybuprocaine hydrochloride solution. Aqueous humor samples were frozen on dry ice immediately after sampling and stored in a $-80^{\circ} \mathrm{C}$ freezer until further processing. As a negative control, aqueous humor was drawn $(100 \mu \mathrm{L})$ from both eyes of seven healthy control animals, after sacrifice. The negative control animals did not receive any topical treatment nor anesthetics. Aqueous humor was drawn within 10 minutes after sacrificing of 
the rabbits. A total of thirteen samples was collected (one sample was lost during processing).

Aqueous humor samples were measured with the Raman spectrometer followed by HPLC analysis using protocols as described earlier.

Pre-processing of the raw acquired Raman spectrum

In order to extract Raman signal from the raw acquired spectrum, it is necessary to pre-process the acquired spectrum. ${ }^{23}$ Cosmic ray spikes, randomly generated due to cosmic radiation, were replaced by the average intensity from the neighboring frames. A partial polynomial fitting method combined with the morphology approach of Perez-Pueyo et al. ${ }^{24}$ was used to remove instrumental noise. First, spectra were dissected in different zones. Zones that only contain background fluorescence were used to calculate the polynomial function coefficients. The zone that contains the water-peak $\left(1550 \mathrm{~cm}^{-1}\right.$ to $\left.1650 \mathrm{~cm}^{-1}\right)$ was excluded from the fitting calculation. The achieved polynomial function was applied on the full spectrum $\left(400 \mathrm{~cm}^{-1}\right.$ to $\left.1700 \mathrm{~cm}^{-1}\right)$ to remove the fluorescence background. Hereafter, the morphology-based approach was applied to eliminate instrumental noise.

In short, our pre-processing procedure are as follows: first, manual cosmic ray removal before any further treatment (figure S1,1). Second, averaging of the frames to minimize the fluctuations. Third, applying partial polynomial $\left(5^{\text {th }}\right.$ degree $)$ fitting on the averaged spectrum for subtraction of fluorescence (figure S1,3) and fourth, using morphology method to eliminate the instrumental noise (figure S1,2). Besides the first step, all procedures are processed by a self-developed MATLAB program (Version 2017b, The Mathworks Inc., Natick, MA, United States). Furthermore, all samples were normalized by dividing ketorolac related peaks by their water-peak $\left(1642 \mathrm{~cm}^{-1}\right)^{25}$ correcting for the sample-sample variation.

Statistical analysis

Statistical analysis was performed using GraphPad Prism version 6.01 (GraphPad Software inc. La Jolla, CA, United States). All in vitro pig eye data were analyzed using 2way ANOVA multiple comparison tests. The in vivo rabbit data was analyzed with paired ttests. The in vitro rabbit data was analyzed using unpaired t-tests because the aqueous humor samples came from different rabbits. All data are shown as mean \pm SD.

\section{Results}

Detection of peaks related to ketorolac tromethamine using Raman spectroscopy

Figure 2 shows the Raman spectrum of Acular ${ }^{\mathrm{TM}}$ and PBS. For the multivariate peak analysis, eight high-intensity peaks specific for ketorolac tromethamine were selected: 1002 $\mathrm{cm}^{-1}, 1282 \mathrm{~cm}^{-1}, 1348 \mathrm{~cm}^{-1}, 1432 \mathrm{~cm}^{-1}, 1472 \mathrm{~cm}^{-1}, 1524 \mathrm{~cm}^{-1}, 1568 \mathrm{~cm}^{-1}$, and $1602 \mathrm{~cm}^{-1}$ as shown in figure 2 (upper spectrum). These peaks were not related to additives such as EDTA or BAK (figure S2). The $1602 \mathrm{~cm}^{-1}$ peak overlaps partially with the water-peak 
(1642 $\mathrm{cm}^{-1}$, underlined in both spectra), this peak is not used during further processing. In the following results, the intensity of each individual peak or the average intensity ratio of the seven peaks is plotted and used.

Four background peaks originating from PBS, aqueous humor and cuvette were detected: $930 \mathrm{~cm}^{-1}, 1120 \mathrm{~cm}^{-1}, 1448 \mathrm{~cm}^{-1}$, and $1642 \mathrm{~cm}^{-1}$ (figure 2 lower spectrum). These four peaks were identical in PBS and AH (figure S4). No peak differences were found between aqueous humor from rabbits and pigs (data not shown).

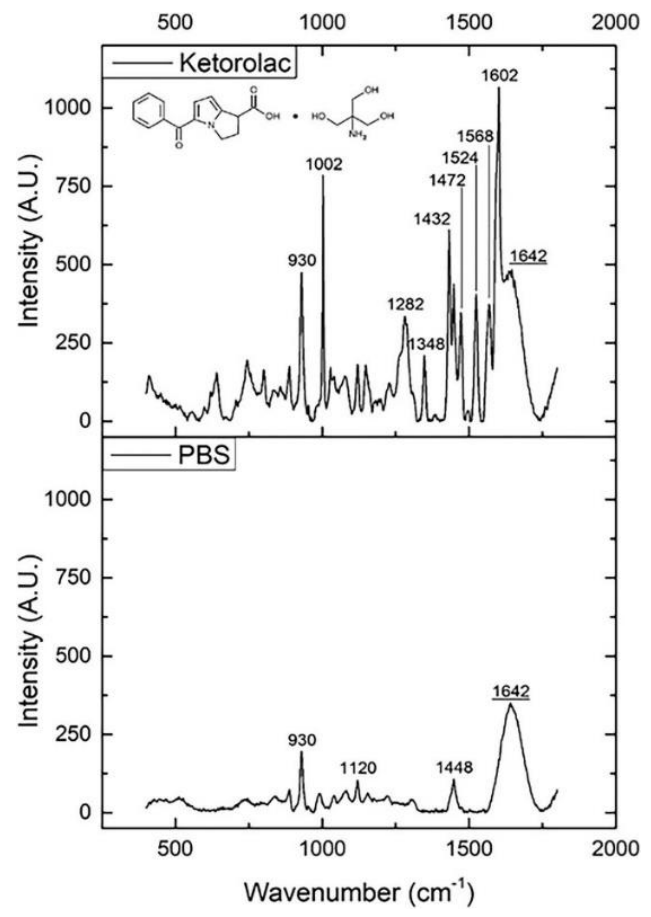

Figure 2. Raman fingerprint spectra of Acular ${ }^{\mathrm{TM}}$ ( $0.5 \%$ ketorolac tromethamine ophthalmic solution) and PBS. The structural formula of ketorolac tromethamine is shown in the frame related to Acular $^{\mathrm{TM}}$. The numbers above the peak represent the corresponding wavenumbers. Baseline correction is applied using polynomial correction. $\mathrm{PBS}=$ phosphate buffered saline, A.U. = arbitrary unit.

Detection of ketorolac tromethamine using the HPLC in aqueous humor of pig eyes

Figure $3 a$ shows the HPLC quantification of ketorolac tromethamine (concentration curve $0.05 \%$ to $1.25 \%$ ), and the ketorolac concentrations in the aqueous humor of pig eyes that were immersed in a similar concentration range as described earlier. Both solutions demonstrate linearity with the tested concentrations, $\mathrm{R}^{2}$ of 0.97 and $\mathrm{R}^{2}$ of 0.88 respectively. When comparing the ketorolac concentration in Acular ${ }^{\mathrm{TM}}$ to the aqueous humor penetrated ketorolac concentration in pig eyes, after submerging for 24 hours in Acular ${ }^{\mathrm{TM}}$, approximately one fourth of the original ketorolac concentration appeared to have penetrated into the aqueous humor. The concentration in the aqueous humor of the pig eye was significantly lower $(\mathrm{p}=0.0012)$ compared to the concentration in which the eye was submerged (figure $3 b$ ). 

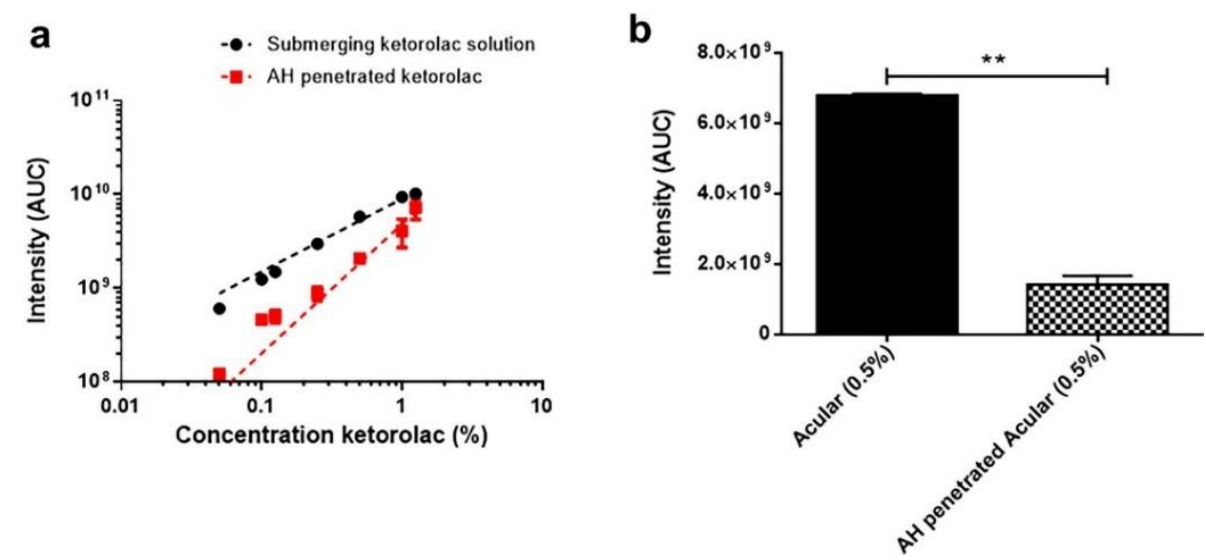

Figure 3. HPLC detection and analysis of (a) a dilution curve of ketorolac tromethamine in PBS (submersion solution) (black) and the detection of penetrated ketorolac in aqueous humor (red). The dashed line represents a logarithmic trendline with a R2 of 0.98 and 0.89 for the black and red points respectively. (b) HPLC analysis of ketorolac tromethamine in Acular ${ }^{\mathrm{TM}}$ and the penetrated concentration in aqueous humor after $24 \mathrm{~h}$. Compared using students t-test, $* * \mathrm{p}=0.0012, \mathrm{n}=3, \mathrm{AH}=$ aqueous humor, $\mathrm{AUC}=$ area under the curve, data is plotted as mean $\pm \mathrm{SD}$.

\section{Detection of ketorolac tromethamine using Raman spectroscopy in pig eyes}

Figure $4 a$ provides a detailed overview of the intensity ratios corresponding to ketorolac peaks of Acular ${ }^{\mathrm{TM}}$ compared to PBS. All peaks related to ketorolac are about five to one hundred and fifty times higher in Acular ${ }^{\mathrm{TM}}$ compared to PBS. After penetration in the aqueous humor of pig eyes, the same ketorolac peaks could be detected, as shown in figure $4 b$. However, no significant difference between the ketorolac and the PBS peak on wavenumber $1348 \mathrm{~cm}^{-1}$ was observed.

When the intensity of the peaks is summarized, the aqueous humor penetrated concentration of ketorolac is about one fourth of the original solution (figure 4c). As expected, there is no difference between PBS and the aqueous humor penetrated PBS signal. These results demonstrate that Raman spectroscopy is able to detect ketorolac solutions after penetration in the aqueous humor.

Figure 5 shows a correlation between the Raman signal and HPLC signal from the concentration curve of aqueous humor penetrated ketorolac in pig eyes. This resulted in Pearson's coefficient of 0.89 with a $\mathrm{R}^{2}$ of 0.79 using correlation on the log-log scale.

Figure 6 demonstrates the quantitative potential of confocal Raman spectroscopy. Figure $6 a$ shows a linear relationship between the concentration and the observed signal for three different set-ups. 
a
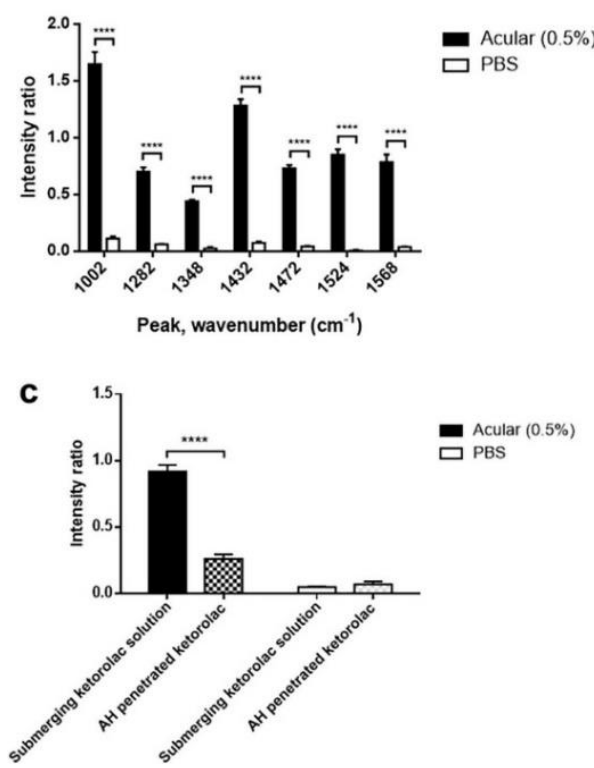

b

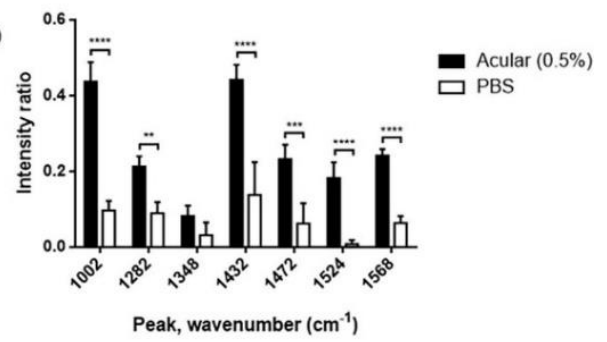

Figure 4. Raman detection of ketorolac in aqueous humor of pig eyes. (a) Peaks corresponding to AcularTM compared to PBS and (b) after penetration in aqueous humor of pig eyes $(n=3)$, row comparison using Tukey's multiple comparisons test. (c) Averaged intensity ratios from Acular ${ }^{\mathrm{TM}}$ and $\mathrm{PBS}$ as the submerging solution and after aqueous humor penetration in pig eyes. Exposure time $60 \mathrm{~s}, 3$ frames averaged, $785 \mathrm{~nm}$ laser. Peaks normalized by dividing the intensity of each peak with the intensity of the peak at $1,642 \mathrm{~cm}^{-1}$, $\mathrm{n}=3 . * *=\mathrm{p}<0.01, * * *=\mathrm{P}<0.001, * * * *=\mathrm{P}<0.0001$. AH, aqueous humor, data is plotted as mean $\pm \mathrm{SD}$.

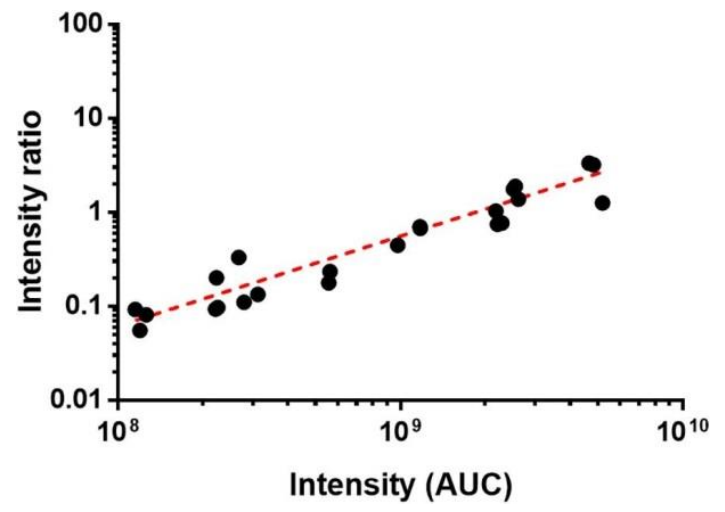

Figure 5. Correlation between Raman signal (y-axis) and the HPLC signal ( $\mathrm{x}$ axis) of aqueous humor from pig eyes submerged in ketorolac dilutions. The dashed line represents a logarithmic trendline. Pearson's $\mathrm{r}$ of 0.89 and a $\mathrm{R}^{2}$ of 0.79 , every dot represents one eye $(n=3$ per concentration). AUC $=$ area under the curve.

The limit of detection of the ketorolac dilution curve ( $0.05 \%$ to $5.0 \%$ ) (black line) lies on an intensity ratio of $0.05 \pm 0.003$, which is lower than the background signal of aqueous humor detected in a cuvette (red line) $(0.07 \pm 0.02)$. The limit of detection of aqueous humor detected with a Jena lens (green line) or a Gonio lens (blue line) lies, however, about nine times higher $(0.45 \pm 0.03$ and $0.34 \pm 0.09$, respectively). 
Figure $6 b$ shows the response of the Raman system with three different set-ups. Different intensity ratios when comparing Acular ${ }^{\mathrm{TM}}$ to PBS have been observed. When measuring Acular ${ }^{\mathrm{TM}}$ and $\mathrm{PBS}$ in a cuvette, a clear difference is visible with low background. When the ketorolac concentration in aqueous humor of pig eyes is measured in a cuvette, the intensity ratio of Acular ${ }^{\mathrm{TM}}$ is lower, but the background has slightly increased. Aqueous humor in the anterior chamber, measured using the Jena lens does not show a difference between an Acular ${ }^{\mathrm{TM}}$ submerged pig eye and a PBS treated pig eye due to high background noise. When ketorolac is detected in aqueous humor in the anterior chamber using the Gonio lens, a high background is observed; however, the Gonio lens is capable to distinguish ketorolac from the PBS samples.
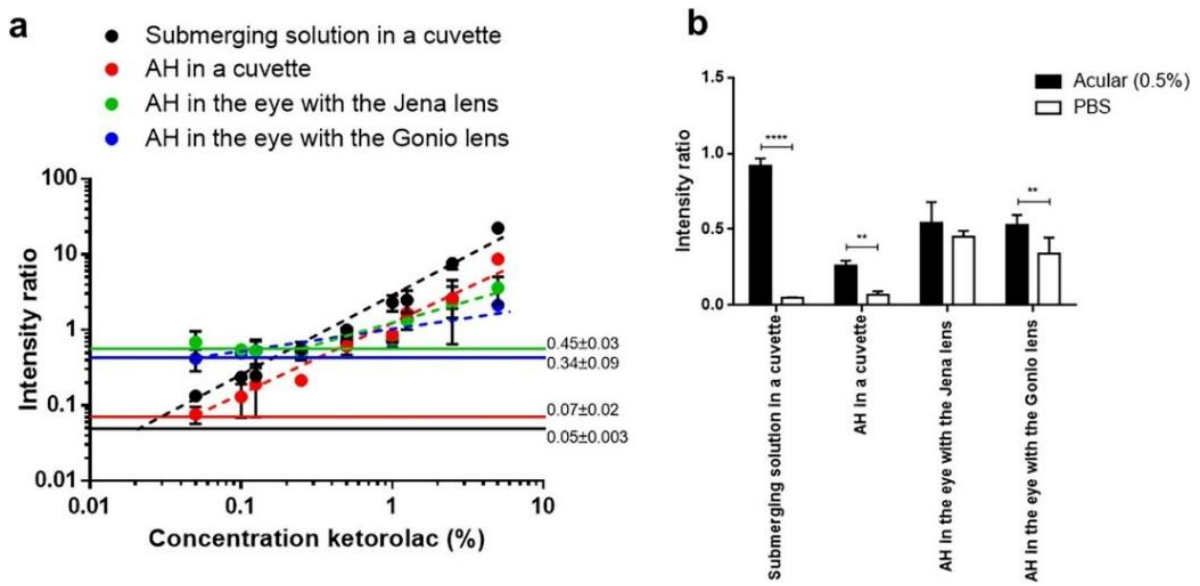

Figure 6. In vitro detection of ketorolac in pig eyes using three different set-ups (aqueous humor in a cuvette, aqueous humor in the anterior chamber with the Jena lens and aqueous humor in the anterior chamber with the Gonio lens) and the control situation (submerging fluid in a cuvette). (a) Dilution series of ketorolac $(0.05 \%$ to $5.0 \%)$ were measured with the Raman spectrometer. Black dots represent the ketorolac tromethamine dilution measured in a cuvette. Red dots represent aqueous humor of pig eyes submerged in corresponding ketorolac tromethamine dilutions after paracentesis measured in a cuvette. The green dots represent measurements on the pig eye, in the anterior chamber with the Jena lens, and the blue dots represent measurement on the eye into the anterior chamber with the Gonio lens. The dashed-lines are drawn as guide to the eyes whereas the solid lines provide the limit of detection. (b) Bar graph with the response of the detection of Acular ${ }^{\mathrm{TM}}$ and PBS. The fluids have been measured in a cuvette, in the aqueous humor using a cuvette, or in the eye with the Jena lens, or in the eye with a Gonio lens. Samples are compared using ANOVA multiple comparison tests, $* \mathrm{P}<0.05, * * \mathrm{P}<0.01, * * * \mathrm{P}<0.001$ and $* * * * \mathrm{p}<0.0001, \mathrm{n}=3$ per point. $\mathrm{AH}=$ aqueous humor, $\mathrm{AC}=$ anterior chamber, data is plotted as mean $\pm \mathrm{SD}$.

\section{In vivo detection of ketorolac in rabbit eyes}

Figure $7 a$ shows that the Gonio lens was able to measure aqueous humor in the anterior chamber of living rabbits; however, no difference between treated (Acular ${ }^{\mathrm{TM}}$ ) and non-treated (BSS) rabbit eyes was observed. When the Gonio lens was not properly aligned on the eye, the lens, or the cornea was measured, as shown in figure $7 b$ and figure $7 c$ 
respectively. In none of the in vivo measurements a significant difference between the treated eye (OD) and the control eye (OS) was observed.

The Raman spectra show the wavenumber $(671 \mathrm{~nm})$ signals corresponding to the location in the eye. When measuring specifically in the aqueous humor, a broad peak is visible between $3000 \mathrm{~cm}^{-1}$ and $3700 \mathrm{~cm}^{-1}$ (figure $7 a$, lowest frame). The lens shows a narrow peak at $2900 \mathrm{~cm}^{-1}$ and a broad one between $3000 \mathrm{~cm}^{-1}$ and $3700 \mathrm{~cm}^{-1}$ (similarly to the peak for aqueous humor) (figure $7 b$ lowest frame). The cornea expresses a high peak at $2900 \mathrm{~cm}^{-1}$ and a broad peak between $3000 \mathrm{~cm}^{-1}$ and $3700 \mathrm{~cm}^{-1}$ (figure $7 c$ lowest frame).

a
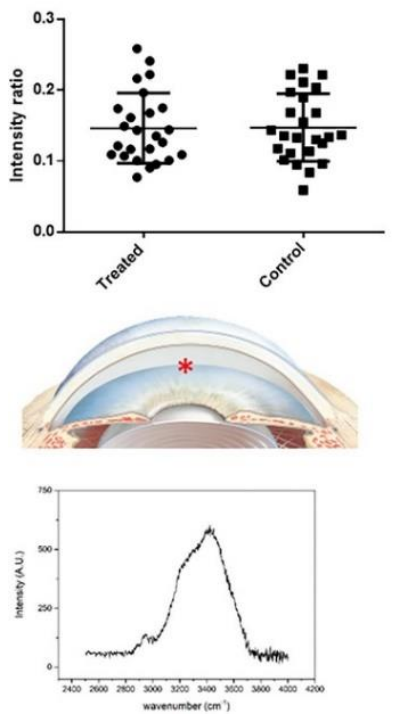

b
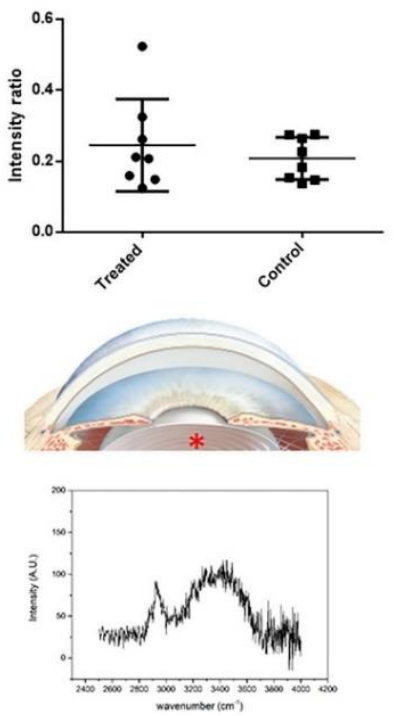

c
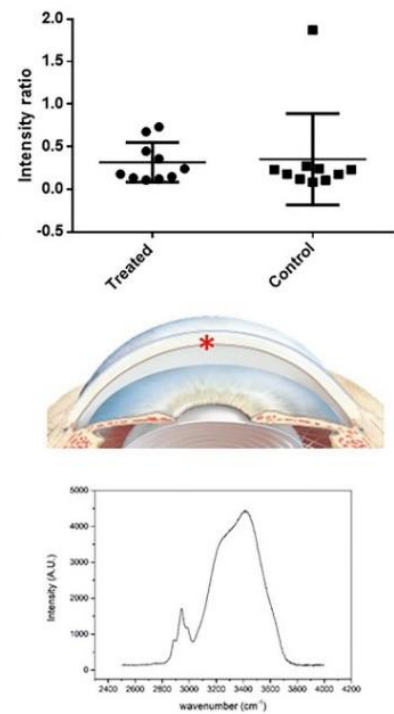

Figure 7. In vivo detection of ketorolac in rabbit eyes. (a) Detection in aqueous humor when the Gonio lens is properly aligned on the eye. The asterisk shows the location of focus and the Raman spectrum provides the high wavenumber $(671 \mathrm{~nm})$ measurement to assure correct location in the eye. (b) Measurement on the lens or (c) on the cornea, due to misalignment of the Gonio lens. Treated eye received three times a day $50 \mu \mathrm{L}$ Acular ${ }^{\mathrm{TM}}$, and the control eye was treated with $50 \mu \mathrm{L}$ BSS (pH7.4). Paired tests have been executed to test difference between treated and control eyes. Each dot represents one measurement at one rabbit, data is plotted as mean $\pm \mathrm{SD}$.

\section{Ketorolac tromethamine detection in aqueous humor of rabbits ex vivo}

Figure 8 shows the ex vivo detection of the ketorolac concentration in aqueous humor from rabbits, measured in a cuvette by Raman and via HPLC. As shown in figure 8a, a significant difference $(\mathrm{p}=0.0017)$ was observed between Acular ${ }^{\mathrm{TM}}$ treated eyes and nontreated eyes when the ketorolac was measured using Raman spectroscopy. However, due to the large inter-sample variation, no exact concentration could be calculated and linked to individual measured aqueous humor samples measured by our Raman spectroscopic system. 
Figure $8 b$ shows the detection of the concentration ketorolac from aqueous humor of rabbits using HPLC. These results also show significant difference $(\mathrm{p}<0.0001)$ between the treated and untreated eyes. When all days are combined, the average drug concentration was $927 \mathrm{ng} / \mathrm{mL} \pm 430 \mathrm{ng} / \mathrm{mL}$ (mean $\pm \mathrm{SD}$ ), a maximum detected concentration of 2236 $\mathrm{ng} / \mathrm{mL}$ and a minimum detected concentration of $63 \mathrm{ng} / \mathrm{mL}$ in the treated eyes. The control eyes did not show any signal above noise level when detected with HPLC. No clear correlation between the Raman signal and HPLC concentration could be found with these low concentrations.
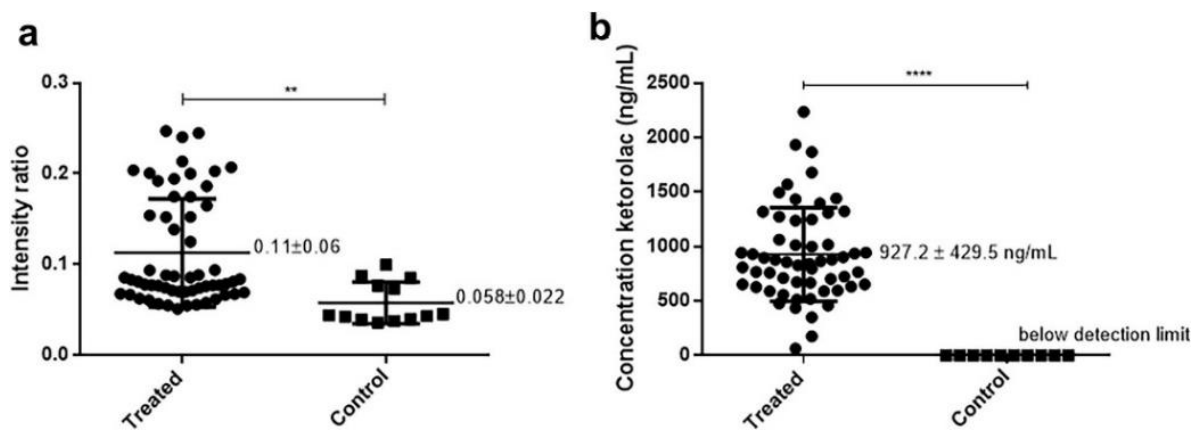

Figure 8. Ex vivo detection of ketorolac tromethamine in aqueous humor from rabbit eyes. General grouped difference between the aqueous humor of treated eyes and control (non-treated) eyes measured with (a) Raman spectroscopy and (b) HPLC. $* * \mathrm{p}<0.01, * * * * \mathrm{p}<0.0001$. Each dot represents one sample from one rabbit, data tested using student t-test and is plotted as mean $\pm \mathrm{SD}, \mathrm{n}_{\text {treated }}=58$ and $\mathrm{n}_{\text {control }}=13$.

\section{Discussion}

The aim of the study was to evaluate the quantitative use of Raman spectroscopy for the in vivo detection of drug levels in the anterior chamber of the eye. Firstly, we needed to optimize the method to analyze our Raman data. The probability of Raman scattering is much lower than intrinsic fluorescence emission in biological samples. Therefore, in order to extract Raman signal from the raw acquired spectrum, it was necessary to pre-process the acquired spectrum. ${ }^{23}$ Furthermore, cosmic ray spikes, randomly generated due to cosmic radiation, which affected different wavenumbers each time, needed to be removed. ${ }^{23,26}$ For the latter, there are two approaches described in the literature. ${ }^{26,27}$ In this study, we went for the easiest method, by replacing the intensities of the cosmic peaks with the average from the neighboring frames (left and right from the ray) as suggested by Zhang et al. ${ }^{26}$

Several approaches have been proposed to minimize the influence from the background fluorescence. ${ }^{13}$ The most accepted method for background subtraction is polynomial fitting but as mentioned by Byrne et al. no standardized protocols are available. ${ }^{13}$ Zhao et al. introduced an automated polynomial background subtraction method for biomedical applications, which could subtract the background fluorescence. ${ }^{28}$ Zhang et al. developed an automated method for fluorescence background subtraction named "automatic pre-processing method for Raman imaging data set (APRI)". ${ }^{26}$ However, 
previous methods encountered difficulties when handling spectrums containing instrumental noise. In some in vivo experiments, the contribution from instrumental noise is inevitable and cannot be neglected, thus affecting the conventional polynomial methods. Hence, further treatments have been developed to eliminate the instrumental noise. PerezPueyo et al. introduced a morphology-based baseline removal method for Raman spectrums. ${ }^{24}$ It employs Tophat filtering using basic operations as dilation and erosion to filter the features beyond or below a pre-set threshold, thereby removing the instrumental noise. In this study, we used a partial polynomial fitting method combined with the morphology approach of Perez-Pueyo et al. to remove instrumental noise. ${ }^{24}$ Hereafter we normalized all samples by dividing ketorolac related peaks by their water-peak $\left(1642 \mathrm{~cm}^{-}\right.$ $\left.{ }^{1}\right),{ }^{25}$ thereby correcting for the sample-sample variation. Overall, we created a solid method to remove hardware related shifts from Raman data.

After we optimized the analysis for Raman spectroscopy, we first confirmed the four ketorolac tromethamine peaks described by Elshout et al. ${ }^{15}$ and then identified four additional peaks specific for ketorolac. We observed that the $1602 \mathrm{~cm}^{-1}$ peak partially overlaps with the water-peak $\left(1642 \mathrm{~cm}^{-1}\right)$; therefore, this peak was ignored during processing.

During the experiment, we examined buffered saline solutions (PBS and BSS), aqueous humor from pig eyes and aqueous humor from rabbit eyes. Although, there are differences between the buffered solutions and aqueous humor, the Raman signal was identical, as shown in supplementary figure $S 4$, and also did not affect the HPLC results. In the pig model, a concentration range of dissolved ketorolac was used and Acular ${ }^{\mathrm{TM}}$ was used as a positive control, whereas only Acular ${ }^{\mathrm{TM}}$ was used in the rabbit model. No differences were found in Raman signal, nor have we observed shifted peaks during the experiments. Although we expected that the Raman signals might be affected due to the additives, we did not observe any interference of the additives on the ketorolac signal (figure S2). Neither have we observed influences of the anesthetics on the Raman signal in the in vivo experiment (figure S5). However, we observed superior penetration of dissolved ketorolac in PBS compared to the commercially available solution (Acular ${ }^{\mathrm{TM}}$ ) (figure S6). The $\mathrm{pH}$ of both solutions was similar (pH 7.4) but the osmolality differs, i.e. Acular ${ }^{\mathrm{TM}}$ displays an osmolality of $290 \mathrm{mOsmol} / \mathrm{kg}^{29}$ whereas dissolved ketorolac tromethamine in PBS has a (theoretical) osmolality of $330 \mathrm{mOsmol} / \mathrm{kg}$. This could explain the higher ocular penetration by the dissolved ketorolac solution. Lee et al. also found osmolality to be a relatively large influencer of ocular penetration in studying penetration of topically applied Atenolol. ${ }^{30}$ However, in our experiment we used post mortem tissue in which the cellular membranes, and tight junctions between the cells are affected and in which clearance of the drug is hampered. Due to submerging of completely enucleated eyes more scleral diffusion is expected leading to increased intraocular drug concentrations as compared to eye drops. ${ }^{31,32}$ Furthermore, the long contact time of 24 hours also enhanced the intraocular drug concentration. The detection of ketorolac in the in vitro model was also challenging 
due to corneal haze in the stromal layers after leaving the eyes in the buffered solutions (figure S3), high background signals were detected which disturbed the Raman measurements. This resulted in high background noise signals that were more than three times higher (when normalized), compared to regular cuvette measurements (figure 6b).

The in vivo animal model represents a realistic clinical situation of an eye drop scheme. The detection of the ketorolac concentration is in line with previously published data. Ling et al. found a $\mathrm{C}_{\max }$ of $1905 \mathrm{ng} / \mathrm{mL} .{ }^{33}$ Although we were not looking for a $\mathrm{C}_{\max }$, our highest detected concentration was $2236 \mathrm{ng} / \mathrm{mL}$. The mean ketorolac concentration in our experiment was $927 \mathrm{ng} / \mathrm{mL} \pm 430 \mathrm{ng} / \mathrm{mL}$, whereas an average concentration of 1079 $\mathrm{ng} / \mathrm{mL} \pm 882 \mathrm{ng} / \mathrm{mL}$ is found in human eyes, when instilling Acular ${ }^{\mathrm{TM}}$ eye drops four times a day, two days pre-surgery. ${ }^{34}$

In our Raman system, two different lenses were compared: a Jena lens and a Gonio lens. Since we used a f60 lens in front of the Gonio lens (the Gonio lens itself does not provide any focus power), better focus was achieved compared to use of the Jena lens. The f60 lens has a smaller numerical aperture, which provides a longer integration length. Second, based on the safety point of view, the Gonio lens prevent the laser from direct illumination on the retina, preventing it for the possible light damage. The excitation laser directly illuminates the retina in the Jena lens setup, it limits the common performance improvement methods like raising the laser power or increasing integration time. Besides, we observed a specific drug related difference when we used a Gonio lens. As such, we continued the experiment in vivo only with the Gonio lens. In vivo the average background signal in the aqueous humor was lower $(0.15 \pm 0.05)$ (figure $7 a$, control eye) compared to in vitro signal ( $0.34 \pm 0.09$, Gonio lens, PBS) (figure 6). Hence, we tend to conclude that the corneal haze was affecting the signal. However, the drug concentration in the aqueous humor was too low to detect using in vivo Raman spectroscopy. Another important factor affecting the sensitivity of Raman spectroscopy is the exposure time during the measurement. All in vitro samples have been exposed 60 seconds for 3 frames, whereas the in vivo exposure was 30 seconds with 2 frames. We lowered the exposure time and the number of frames in vivo to assure safety of the technique in the rabbits. Because of the limited number of frames the threshold of the intensity ratio $(0.15 \pm 0.05)$ was higher in vivo (figure $7 a$, control eye) compared to in vitro intensity ratio $(0.11 \pm 0.06)$ (figure $8 a$, control eye). Besides the lower number of frames, also a shorter exposure time may lead to a decreased Raman signal. ${ }^{35}$ Due to the large inter-measurement-variations, the standard deviation in the Raman experiment was too large to clearly quantify the in vivo samples. The variation with the HPLC was much smaller resulting in a detection accuracy of nanograms per milliliter. Due to large variations in the in vivo fingerprint signals, no correlation could be found using the rabbit samples, whereas there is a clear correlation with Raman spectroscopy and HPLC when measuring higher drug concentrations from the in vitro pig eyes (figure 7). Overall, multiple factors affect the readout and it is difficult to select one parameter causing low sensitivity with Raman spectroscopy. In vitro samples 
have been centrifuged to remove proteins, which could be a reason for higher signal during the in vitro measurements compared to the in vivo measurements. Furthermore, the cornea consists of a $500 \mu \mathrm{m}$ thick stroma, which could scatter or absorb Raman scatter on its way through. Our experiment also shows that the conditions of the cornea could affect the Raman signal sensitivity (corneal haze). Finally, the temperature might also slightly influence the Raman intensity both for the target components and backgrounds. The cuvette samples and in vitro samples were tested at room temperature (about $22^{\circ} \mathrm{C}$ ) while the temperature of in vivo measurement is around $35^{\circ} \mathrm{C}$ in a rabbit eye. It is noticeable that all factors together lower the sensitivity of Raman spectroscopy.

To increase sensitivity in a Raman system, laser power could be increased. The problem however, with increasing laser power in the eye, is the irreversible damage of the photosensitive layers that could occur. In order to protect the eyes, we used a laser power of $26 \mathrm{~mW}$, which is relatively low compared to laser powers which are used on skin (80 $\mathrm{mW}),{ }^{36}$ or on cells or tissue sections $(60 \mathrm{~mW}) .{ }^{37}$ For in vitro detection of corneal biomarkers an intensity of $300 \mathrm{~mW}$ is used, ${ }^{38}$ and even $1 \mathrm{~W}$ is used to create virtual crosssections of intact eye tissue without dependence on tissue processing. ${ }^{39}$ According to Marro et al., laser powers up to $100 \mathrm{~mW}$ are safe to use on retina organotypic cultures (in vitro). ${ }^{40}$ Besides laser power, the wavelength and the exposure time are of importance for the prevention of tissue damage. In our study, we used ketorolac tromethamine. Other ocular drugs, however, may have a stronger Raman signal and can be easier to detect in the anterior chamber. All these parameters make Raman spectroscopy a challenging technique. Furthermore, as mentioned by Byrne et al., ${ }^{13}$ there is no common accepted manner to correct Raman data. Due to hardware influences and sample-to-sample variation, every Raman spectrometer needs its own corrections.

\section{Conclusion}

In this study, we show the value of Raman spectroscopy for the detection of drugs in the anterior chamber of the eye. As expected, the sensitivity and the limit of detection of the HPLC are much higher compared to Raman spectroscopy. However, Raman spectroscopy shows unique potential as a non-invasive technique for real time biomedical analysis. We found good correlation between Raman spectroscopy and HPLC for in vitro detection of drugs. Unfortunately, our Raman spectroscopic system is not yet able to detect a clinically relevant dose of ketorolac tromethamine in the anterior chamber of rabbits in vivo. More research should be conducted to increase the sensitivity of Raman spectroscopy while still using low, non-damaging, laser powers. 


\section{References}

1. $\quad$ C. L. DeVane, and W. J. Jusko, "Dosage regimen design," Pharmacol Ther 17(2), 143-163 (1982).

2. K. Buckley, and A. G. Ryder, "Applications of Raman Spectroscopy in Biopharmaceutical Manufacturing: A Short Review," Appl Spectrosc 71(6), 1085-1116 (2017).

3. R. D. Schoenwald, "Ocular drug delivery. Pharmacokinetic considerations," Clin Pharmacokinet 18(4), 255-269 (1990).

4. U. B. Kompella, R. S. Kadam, and V. H. Lee, "Recent advances in ophthalmic drug delivery," Ther Deliv 1(3), 435-456 (2010).

5. M. Hornof, E. Toropainen, and A. Urtti, "Cell culture models of the ocular barriers," Eur J Pharm Biopharm 60(2), 207-225 (2005).

6. C. V. Raman, and K. S. Krishnan, "A New Type of Secondary Radiation," Nature 121(501-502 (1928).

7. N. J. C. Bauer, "Potential applications of confocal Raman spectroscopy in ophthalmology," in Ophthalmology, p. 160, Datawyse, Maastricht (1999).

8. R. J. Erckens, "Development of the Raman spectroscopic technique for in vivo applications in the eye," in Ophthalmology, p. 140, Unigraphic, Maastricht (2001).

9. A. A. Gowen et al., "On the feasibility of near infrared spectroscopy to detect contaminants in water using single salt solutions as model systems," Talanta 131(609-618 (2015).

10. B. Li et al., "Rapid characterization and quality control of complex cell culture media solutions using raman spectroscopy and chemometrics," Biotechnol Bioeng 107(2), 290-301 (2010).

11. D. A. Long, "Handbook of Raman spectroscopy. From the research laboratory to the process line," in Journal of Raman Spectroscopy I. R. L. a. H. G. M. Edwards., Ed., pp. 91-91, Marcel Dekker, New York and Basel (2004).

12. S. H. Yun, and S. J. J. Kwok, "Light in diagnosis, therapy and surgery," Nat Biomed Eng 1((2017).

13. H. J. Byrne et al., "Spectral pre and post processing for infrared and Raman spectroscopy of biological tissues and cells," Chem Soc Rev 45(7), 1865-1878 (2016).

14. D. F. Willemse-Erix et al., "Optical fingerprinting in bacterial epidemiology: Raman spectroscopy as a real-time typing method," J Clin Microbiol 47(3), 652-659 (2009).

15. M. Elshout et al., "Detection of Raman spectra in ocular drugs for potential in vivo application of Raman spectroscopy," J Ocul Pharmacol Ther 27(5), 445-451 (2011).

16. N. J. Bauer et al., "Non-invasive assessment of ocular pharmacokinetics using Confocal Raman Spectroscopy," J Ocul Pharmacol Ther 15(2), 123-134 (1999).

17. M. S. Borchert, M. C. Storrie-Lombardi, and J. L. Lambert, "A noninvasive glucose monitor: preliminary results in rabbits," Diabetes Technol Ther 1(2), 145-151 (1999).

18. C. C. Pelletier, J. L. Lambert, and M. Borchert, "Determination of glucose in human aqueous humor using Raman spectroscopy and designed-solution calibration," Appl Spectrosc 59(8), 1024-1031 (2005).

19. T. Sideroudi et al., "Non-contact detection of ciprofloxacin in a model anterior chamber using Raman spectroscopy," J Biomed Opt 12(3), 034005 (2007).

20. K. Hosseini et al., "Non-invasive monitoring of commonly used intraocular drugs against endophthalmitis by Raman spectroscopy," Lasers in surgery and medicine 32(4), 265-270 (2003).

21. F. H. M. Jongsma et al., "Confocal Raman spectroscopy system for noncontact scanning of ocular tissues: an in vitro study," 7 (1997).

22. $\quad$ US Pharmacopeia., "USP Ketorolac tromethamine," online (2018).

23. K. Hosseini et al., "Noninvasive detection of ganciclovir in ocular tissue by Raman spectroscopy: implication for monitoring of drug release," J Ocul Pharmacol Ther 18(3), 277-285 (2002).

24. R. Perez-Pueyo, M. J. Soneira, and S. Ruiz-Moreno, "Morphology-based automated baseline removal for Raman spectra of artistic pigments," Appl Spectrosc 64(6), 595-600 (2010).

25. R. J. Erckens et al., "Raman spectroscopy for non-invasive characterization of ocular tissue: Potential for detection of biological molecules," Journal of Raman Spectroscopy 28(5), 293-299 (1997).

26. X. Zhang et al., "Method for Removing Spectral Contaminants to Improve Analysis of Raman Imaging Data," Sci Rep $7(39891$ (2017).

27. S. Li, and L. Dai, "An improved algorithm to remove cosmic spikes in Raman spectra for online monitoring," Appl Spectrosc 65(11), 1300-1306 (2011).

28. J. Zhao et al., "Automated autofluorescence background subtraction algorithm for biomedical Raman spectroscopy," Appl Spectrosc 61(11), 1225-1232 (2007).

29. Allergan Inc., "ACULAR® Prescribing Informaiton," in online, p. 6 (2012).

30. Y. H. Lee, and V. H. L. Lee, "Formulation Influence on Ocular and Systemic Absorption of Topically 
Applied Atenolol in the Pigmented Rabbit," Journal of Ocular Pharmacology 9(1), 47-58 (1993).

31. C. J. F. Bertens et al., "Topical drug delivery devices: A review," Exp Eye Res 168(149-160 (2018).

32. E. M. Del Amo et al., "Pharmacokinetic aspects of retinal drug delivery," Prog Retin Eye Res 57(134185 (2017).

33. T. L. Ling, and D. L. Combs, "Ocular bioavailability and tissue distribution of [14C]ketorolac tromethamine in rabbits," J Pharm Sci 76(4), 289-294 (1987).

34. F. A. Bucci, Jr., and L. D. Waterbury, "Comparison of ketorolac $0.4 \%$ and bromfenac $0.09 \%$ at trough dosing: aqueous drug absorption and prostaglandin E2 levels," J Cataract Refract Surg 34(9), 1509. 1512 (2008).

35. J. C. Lázaro et al., "Optimizing the Raman signal for characterizing organic samples: The effect of slit aperture and exposure time," Spectroscopy 23(2), (2009).

36. L. P. Rangaraju et al., "Classification of burn injury using Raman spectroscopy and optical coherence tomography: An ex-vivo study on porcine skin," Burns (2018).

37. O. Jonas et al., "In vivo detection of drug-induced apoptosis in tumors using Raman spectroscopy," Analyst 143(20), 4836-4839 (2018).

38. Y. Kaji et al., "Raman Microscopy: A Noninvasive Method to Visualize the Localizations of Biomolecules in the Cornea," Cornea 36 Suppl 1(S67-S71 (2017).

39. D. A. Ammar et al., "Imaging the intact mouse cornea using coherent anti-stokes Raman scattering (CARS)," Invest Ophthalmol Vis Sci 54(8), 5258-5265 (2013).

40. M. Marro et al., "Dynamic molecular monitoring of retina inflammation by in vivo Raman spectroscopy coupled with multivariate analysis," J Biophotonics 7(9), 724-734 (2014). 


\section{Supplementary figures}
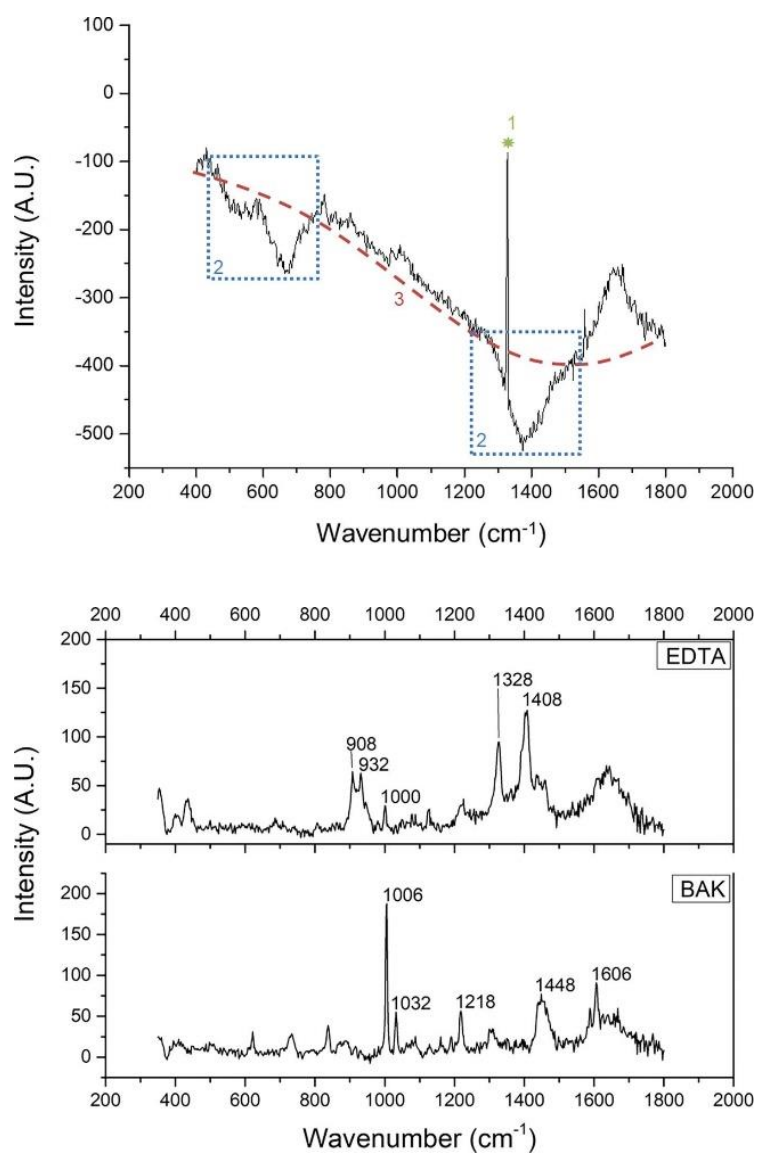

Figure S1. Example of baseline correction of raw Raman spectra. (1) Cosmic ray correction, (2) correction for hardwareinduced errors and (3) $5^{\text {th }}$ degree polynomial correction for background fluorescence. $\mathrm{cm}^{-1}=$ typically centimeters, A.U. $=$ arbitrary unit.

Figure S2. Raman spectrum of the additives in AcularTM. None of the peaks corresponds to the ketorolac spectrum. Only baseline correction is applied on the spectrum using polynomial correction. RAW data. Analyzed using OriginPro 9 64bit e.d. 

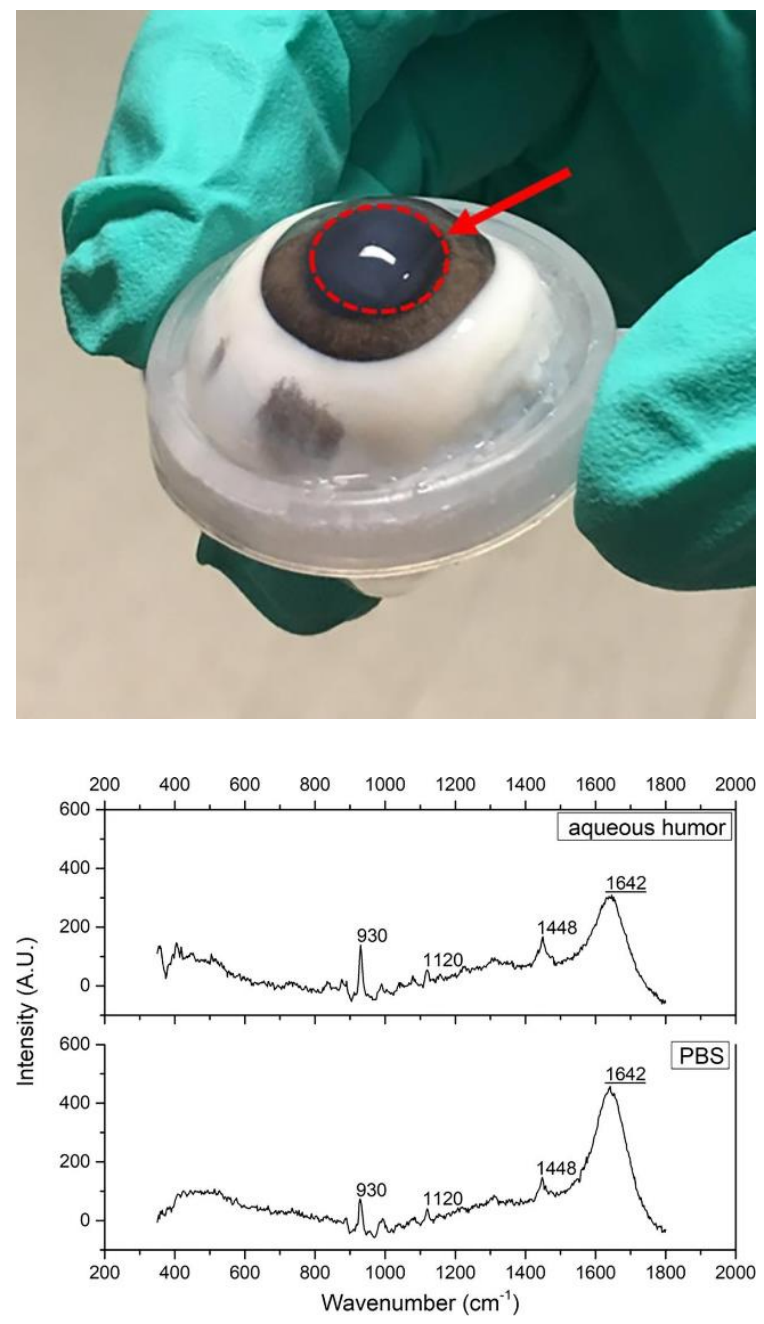

Figure S3. Post mortem pig eye with a clearly visible corneal haze in the stromal layers.

Figure S4. Raman spectrum of PBS and aqueous humor. The peaks in PBS and aqueous humor are similar to each other. Only baseline correction is applied on the spectrum using polynomial correction. RAW data. Analyzed using OriginPro 9 64bit e.d. 


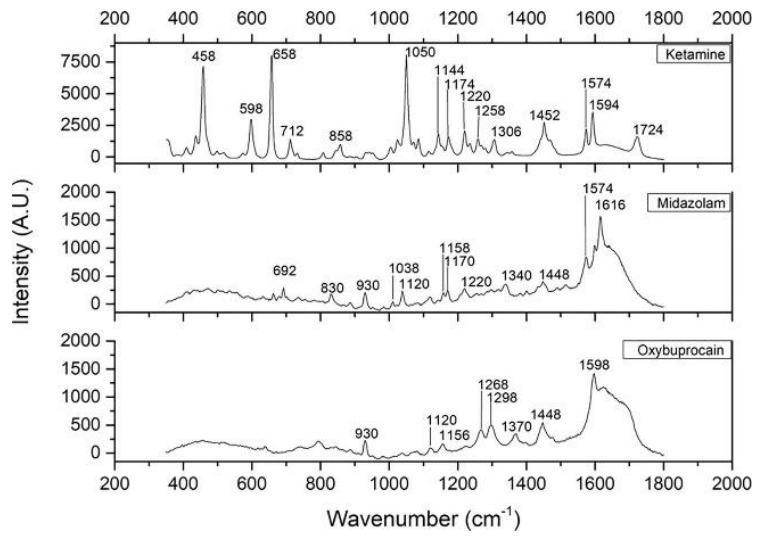

Figure S5. Raman spectrum of ketamine, midazolam, and Oxybuprocaine hydrochloride. Only baseline correction is applied on the spectrum, using polynomial correction. RAW data. Analyzed using OriginPro 9 64bit e.d.
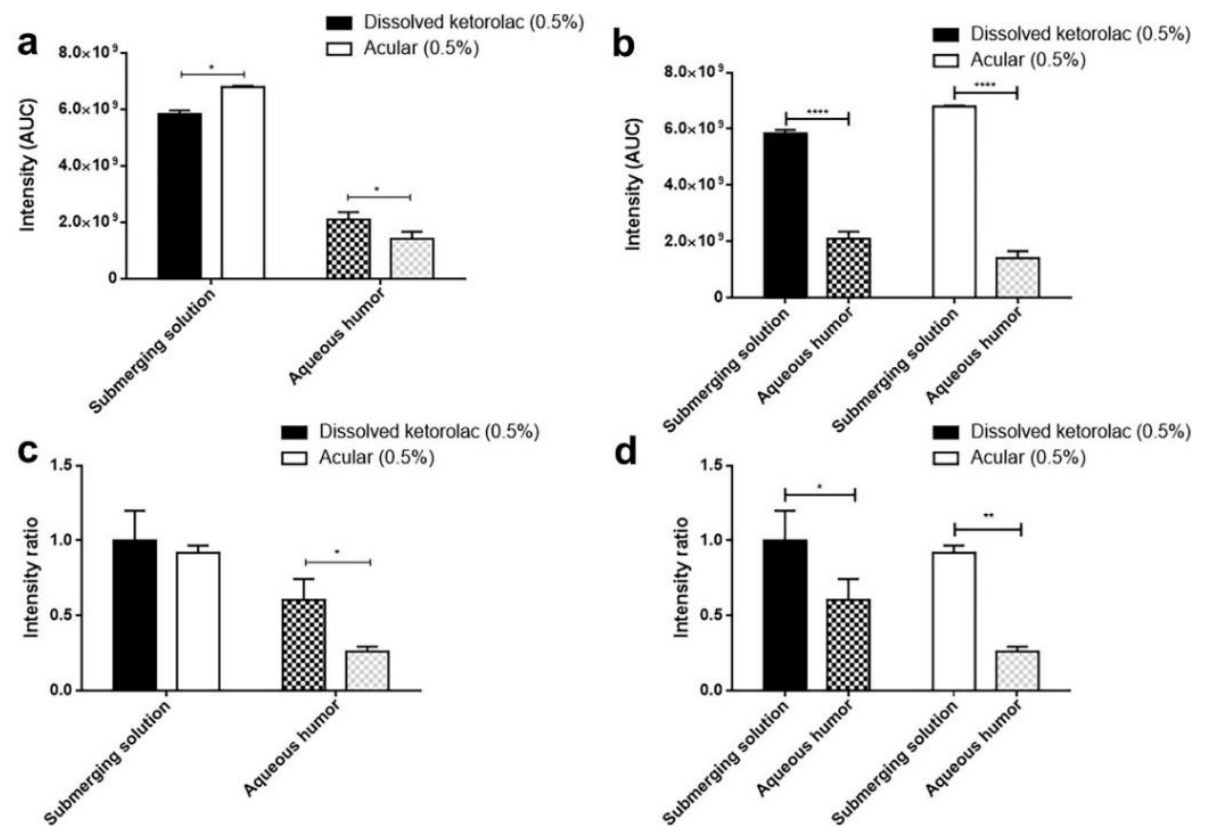

d

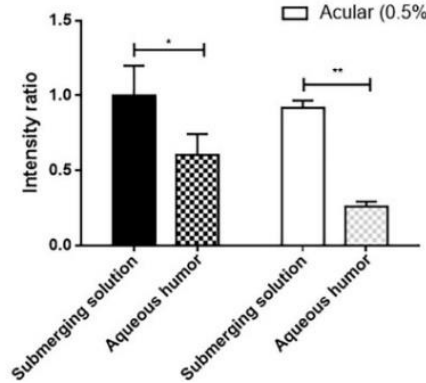

Figure S6. Ocular penetration of dissolved ketorolac compared to Acular ${ }^{\mathrm{TM}}$ in pig eyes. (a) and (b) show HPLC analysis between AcularTM and dissolved ketorolac and graph (c) and (d) show the Raman data (cuvette detection). ${ }^{*} \mathrm{P}<0.05,{ }^{*} \mathrm{P}<0.01,{ }^{* * * *} \mathrm{P}<0.0001 \mathrm{n}=3$ per sample, data is plotted as mean $\pm \mathrm{SD}$. 


\section{Chapter 4a}

Pipeline for the removal of hardware related artifacts and background noise for Raman spectroscopy

Christian J.F. Bertens", Shuo Zhang ${ }^{\#}$, Roel J. Erckens, Frank J.H.M. van den Biggelaar, Tos T.J.M. Berendschot, Carroll A.B. Webers, Rudy M.M.A. Nuijts and Marlies Gijs \# Equal Contribution

MethodsX 2020;7:100883

DOI: 10.1016/j.mex.2020.100883 


\begin{abstract}
Raman spectroscopy is a real-time, non-contact, and non-destructive technique able to obtain information about the composition of materials, chemicals, and mixtures. It uses the energy transfer properties of molecules to detect the composition of matter. Raman spectroscopy is mainly used in the chemical field because background fluorescence and instrumental noise affect biological (in vitro and in vivo) measurements. In this method, we describe how hardware related artifacts and fluorescence background can be corrected without affecting signal of the measurement. First, we applied manual correction for cosmic ray spikes, followed by automated correction to reduce fluorescence and hardware related artifacts based on a partial $5^{\text {th }}$ degree polynomial fitting and Tophat correction. Along with this manuscript we provide a MatLab ${ }^{\circledR}$ script for the automated correction of Raman spectra.
\end{abstract}

\title{
Graphical abstract
}

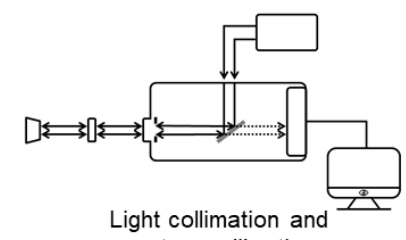
system calibration

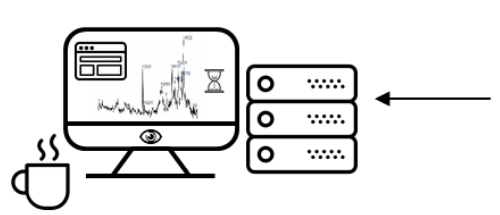

Data processing using: Polynomial_Tophat_background_subtraction_methods.m

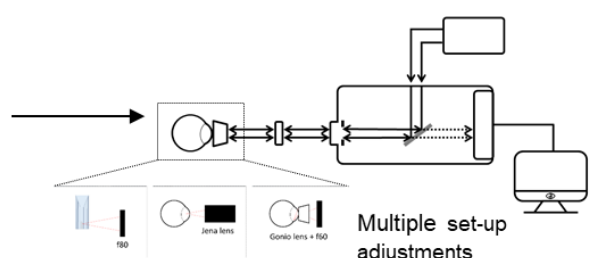

adjustments

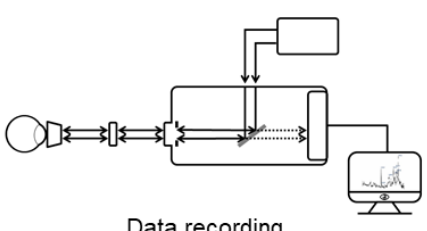

Data recording

\section{Highlights}

- "Polynomial_Tophat_background_subtraction_methods.m" offers an automated method for the removal of hardware related artifacts and fluorescence signals in Raman spectra.

- “Polynomial_Tophat_background_subtraction_methods.m” provides a modifiable MatLab file adjustable for multi-purpose spectroscopy analysis.

- We offer a standardized method for Raman spectra processing suitable for biological and chemical applications for modular confocal Raman spectroscope 


\section{Introduction}

Raman spectroscopy is a vibrational spectroscopic technique, based on an energy transfer between an illuminated sample and the irradiated light. In contrast with e.g. infrared (IR) spectroscopy, which analyses absorbed and transmitted fractions of the light, Raman spectroscopy makes use of scattered radiation. Although the predominant mode of scattered light is elastic Rayleigh scattering, a small proportion ( 1 to $10^{9}$ or $\left.10^{10}\right)$ of the photons is scattered inelastically. These photons shift to a higher or lower energy status resulting in stokes and anti-stokes scattering. ${ }^{1}$

Raman spectra provides both qualitative and quantitative molecular-level information. The basis of the qualitative information is the fingerprint nature of the Raman shift, which is unique to each material. This makes Raman spectroscopy also usable in an aqueous environment $^{2}$, and an interesting and suitable technique for ophthalmic purposes. Raman spectroscopy is a non-contact and non-destructive technique with real-time visualisation, which make it also suitable for in vivo application.

Biological samples often emit fluorescence signals that may interfere with Raman signals since the intensity of the fluorescence emission has a much higher yield than Raman signals. $^{3}$ Further, hardware related artefacts (instrumental noise) are found in Raman spectra. In order to extract Raman signal from the raw acquired spectrum, it is therefore necessary to pre-process the acquired spectra. ${ }^{4}$ As recognized by Byrne et al. no standardized protocols are available for this purpose yet. ${ }^{5}$ Hence, we developed a method to deal with multiple source background influences. This paper guides you through the steps taken to optimize Raman spectra and make them ready for analysis as done in the study from Bertens et al. ${ }^{6}$ For the full data-set of this project we refer to the supplementary data of Zhang et al. ${ }^{7}$

\section{Background of the data processing}

As mentioned earlier, there is no gold standard for the processing of Raman data. Several approaches have been proposed to minimize the influence from background fluorescence. ${ }^{5}$ Raman scattering is an instantaneous effect, whereas fluorescence requires time to occur. If one can switch on and off the detector (or a filter) at a high temporal resolution, fluorescence signal could be prevented from interfering with the Raman signal. However, this is expensive, complicated, and commercially not available. ${ }^{8,9}$ Therefore, the most accepted method for fluorescence background subtraction is polynomial fitting, for which unfortunately no standardized protocols are available (figure 1-3). ${ }^{5}$ Zhao et al. introduced an automated polynomial background subtraction method for biomedical applications, which could subtract the background. ${ }^{10}$ Zhang et al. also developed a proper automated method for fluorescence background subtraction named: "automatic preprocessing method for Raman imaging data set (APRI)". ${ }^{11}$ However, both methods encountered difficulties when handling spectrums containing instrumental noise. In some in vivo experiments, the contribution from instrumental noise is inevitable and cannot be 
neglected, thus affecting the conventional polynomial methods. Hence, further treatments have been developed to eliminate the instrumental noise. Perez-Pueyo et al. introduced a morphology-based baseline removal method for Raman spectrums. ${ }^{12}$ It employs Tophat filtering using basic operations as dilation and erosion to filter the features beyond or below a pre-set threshold, thereby removing the instrumental noise (figure 1-2).

A third influencer affecting Raman spectra are cosmic rays. Cosmic rays create spikes that are randomly generated due to cosmic radiation (figure 1-1). Cosmic rays affected different wavenumbers each time they occur, and can easily be detected by comparing different frames of one measurement. Spikes created by cosmic rays need to be removed before the frames are averaged, else they can be interpreted as peaks. ${ }^{4,11}$

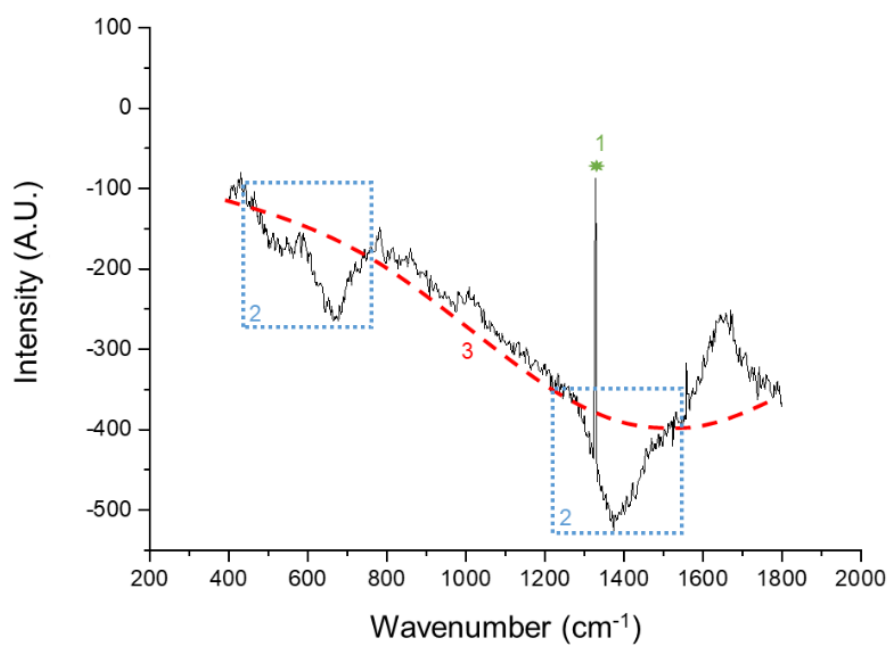

Figure 1. Example of a Raman spectrum. (1, green) Shows a cosmic ray spike, (2, blue) shows instrumental influences on the spectrum, and $(3$, red $)$ shows a $5^{\text {th }}$ degree polynomial fitting for background correction

\section{Materials and Methods}

Materials

- Power conditioner: ONEAC PCm750I, 220-240V, 3.1A, 50/60Hz

- Laser with wavelength $785 \mathrm{~nm}$ : Laser Model SM $785 \mathrm{~nm}$ purchased from Innovative Photonic Solutions; Output Power $50 \mathrm{~mW}$; Bandwidth $9.73 \mathrm{GHz}$

- Laser with wavelength $671 \mathrm{~nm}$ : Laser model: Ignis 671, purchased from Laser quantum; Output Power $100 \mathrm{~mW}$; Bandwidth $30 \mathrm{GHz}$

- Spectrometer: Model HPRM 2500, produced by River Diagnostics International BV. Specifications: 
- Spectral region coverage: $350 \mathrm{~cm}^{-1}-1800 \mathrm{~cm}^{-1}$ with the $785 \mathrm{~nm}$ laser and $\sim 2500 \mathrm{~cm}^{-1}-4000 \mathrm{~cm}^{-1}$ with the $671 \mathrm{~nm}$ laser

- Spectral resolution: $2 \mathrm{~cm}^{-1}$ throughout the spectral region

- Pinhole size: 25 microns

○ Back-illuminated deep-depletion CCD-camera: with 1024 x 128 pixels, air-cooled to $-60^{\circ} \mathrm{C}$. Camera control software included

- Computer: HP Compaq 6200 Pro Microtower with operation system Windows ${ }^{\circledR} 7$ Pro $\mathrm{OA}$

- Jena lens: Planachromat LD 25x/0.5 $\infty / 0(2)$-A, focus length is $10.1 \mathrm{~mm}$

- Mirror: Beam steering mirror assembly, model G063713000

- Melles Griot Shear-plate

- Fibres: Diamond® FC APC/PM 20853190002 for 850nm and FC APC/PM 20871100001 for $630 \mathrm{~nm}$

- GonioLens, Haag-Streit Meridian; CGA1

- $\quad$ Edmund Optics lenses: f60 (60 mm focus point), f80 (80 $\mathrm{mm}$ focus point)

Set-up of the Raman system

A modular confocal Raman spectroscopic system was used in the study. The Raman system was connected via a power conditioner, to prevent power peaks to disturb the measurements and to protect the system. The Raman system is equipped with a diodeemitting laser of $785 \mathrm{~nm}$ with a continuous power of $26 \mathrm{~mW}$, and a $671 \mathrm{~nm}$ diode- emitting laser with a continuous power of $14 \mathrm{~mW}$. Raman spectra were recorded with a highperformance Raman module model 2500 with a charge-coupled device (CCD) operating at $-60^{\circ} \mathrm{C}$. This module introduces the laser light through a diamond optical fiber, shapes and conditions the beam through a pinhole to the measurement stage (figure 3). The emitting light from the spectrometer is collimated using a converging lens (f80 see figure 3-f). Collimation of the light was checked using the Melles Griot shear-plate. The lens was moved along the laser optic axis towards or away from the exit aperture of the spectrometer until the stripes provide a collimated position (figure 2).
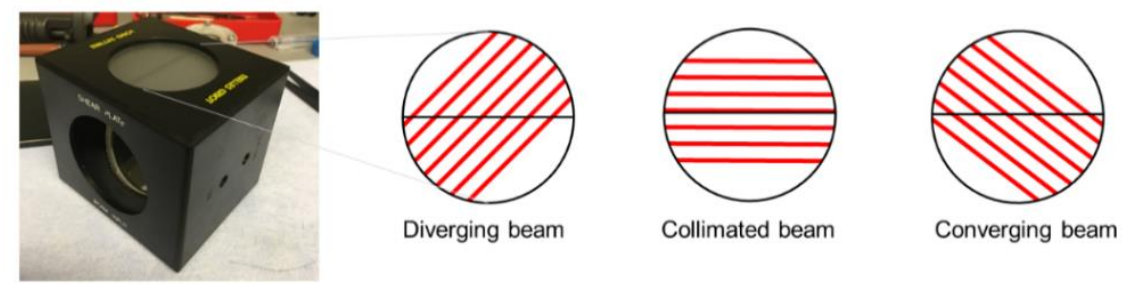

Figure 2. Melles Griot shear plate and the patterns it provides with different types of emitted light.

Three types of sample set-ups were performed: 
- Cuvette set-up (figure 4a)

- In front of the sample, a f80 lens was used when the sample was measured in a Brand ${ }^{\circledR}$ cuvette.

- Jena lens set-up (figure $4 b$ )

- In front of the sample, a long-working-distance microscope objective lens, Jena lens.

- Gonio lens set-up (figure 4c)

- In front of the sample, first a lens with a f60 lens is placed, followed by a Gonio lens. The Gonio lens was connected to the cornea of an eye (in vivo or ex vivo) using topically applied Methoce ${ }^{\circledR} 2 \%$.

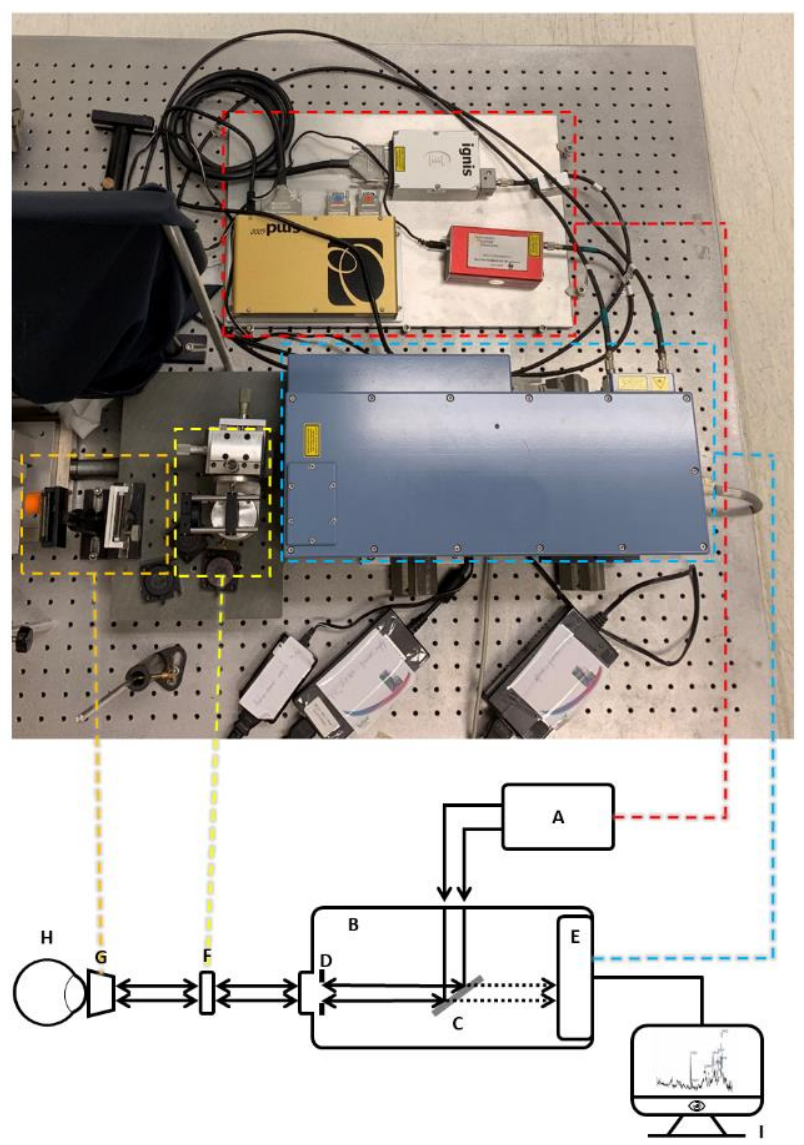

Figure 3. Raman set-up. (A) laser (red dashed region); (B) Raman module (blue dashed region), with (C) filter for Raman scattered light, (D) $25 \mu \mathrm{m}$ pinhole and (E) integrated charge-coupled device (CCD); (F) collimation f80 lens (yellow dashed region); (G) f60 lens with a Gonio (one-mirror) lens, or objective (Jena lens), or a $\mathrm{f} 80$ lens (orange dashed region); $(\mathrm{H})$ sample; and (I) computer (didn't show in the photograph). Arrows indicate direction of (backscattered) laser light; dashed arrows indicate direction of Raman-Scattered light. 


\section{Calibration}

When the laser from the Raman system is collimated, the lens used for the measurement is set in place and the system is calibrated by built-in calibration procedure of the spectrometer. Hereafter, the system is further calibrated by the reference spectrum obtained by the provided National Institute of Standards and Technology (NIST)-standard calibration glass (was provided with the spectrometer). The full calibration was done according to the spectrometer manual. All measurements were performed in the dark.
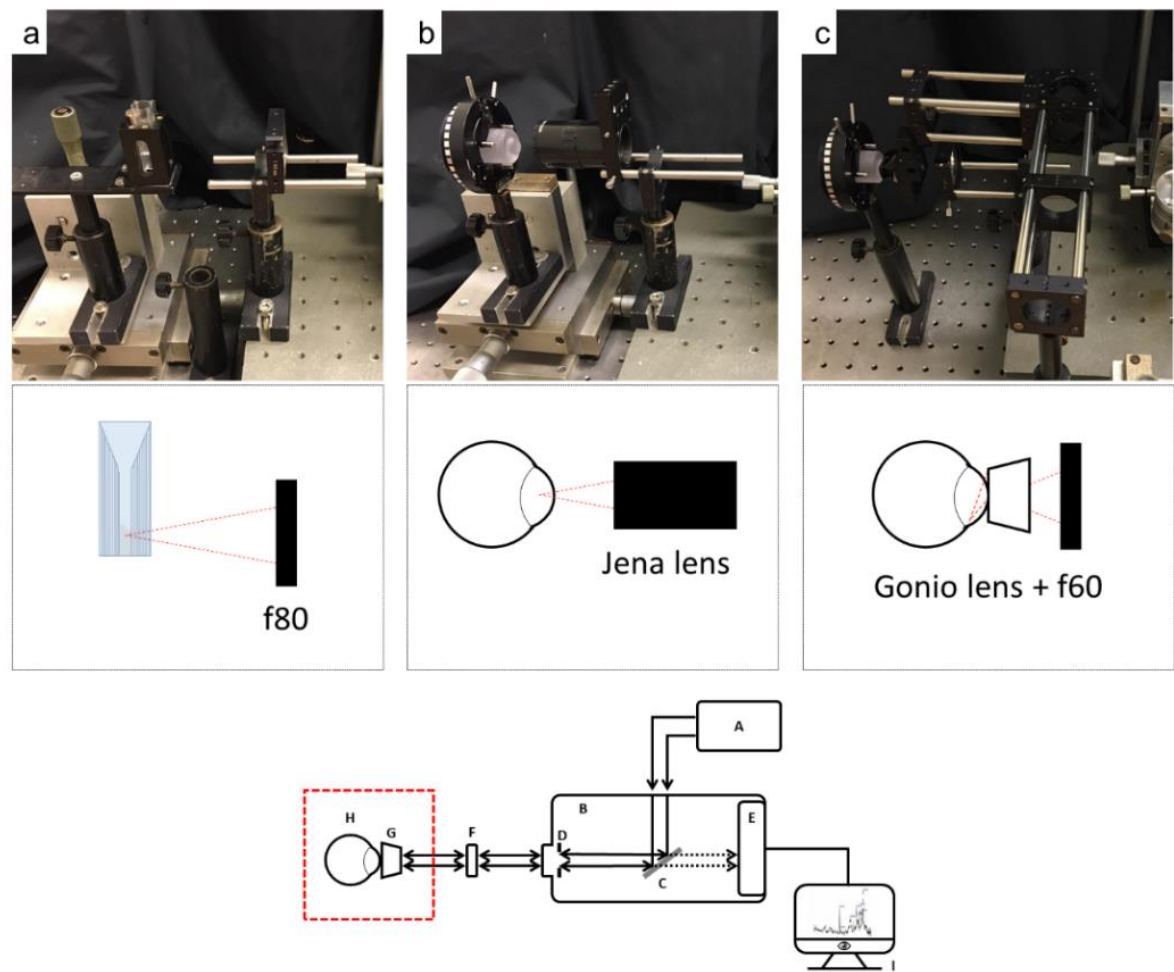

Gonio lens + f60

Figure 4. Details of the focus area of the Raman system (red dashed line in the scheme). (a) The set-up for cuvettes using a 180 lens, (b) Jena lens for focus in the anterior chamber of an eye, and (c) the Gonio lens in combination with a f60 focus lens for focus in the anterior chamber of an eye. The red dashed triangles show the focus position of the set-up.

\section{Positioning}

The location in the sample was determined using the $671 \mathrm{~nm}$ laser, to create a high wave number signal (figure $5 \mathrm{a}$ ). In the eye, the cornea provides a protein peak $\left(2800 \mathrm{~cm}^{-1}-\right.$ $\left.3000 \mathrm{~cm}^{-1}\right)$ followed by a water band $\left(3000 \mathrm{~cm}^{-1}-3800 \mathrm{~cm}^{-1}\right)$. The anterior chamber only has a water band $\left(3000 \mathrm{~cm}^{-1}-3800 \mathrm{~cm}^{-1}\right)$, and the lens has an extra protein peak around 
$3100 \mathrm{~cm}^{-1}$ besides the protein peak located at $2800 \mathrm{~cm}^{-1}-3000 \mathrm{~cm}^{-1}$ and a water peak at $3000 \mathrm{~cm}^{-1}-3800 \mathrm{~cm}^{-1}$. (figure $5 \mathrm{~b}$ ) In a cuvette, when focussed on the cuvette multiple highintensity signals occur (between $2000 \mathrm{~cm}^{-1}-3000 \mathrm{~cm}^{-1}$ ). When focussed on the fluid in the cuvette a water peak occurs $\left(3000 \mathrm{~cm}^{-1}-3800 \mathrm{~cm}^{-1}\right)$.

\section{Data acquisition}

When the laser was correctly positioned, fingerprint-signal of the material was measured with the $785 \mathrm{~nm}$ laser and exported as '.txt' file further processing. An example of a measurement is provided in figure 6.

a
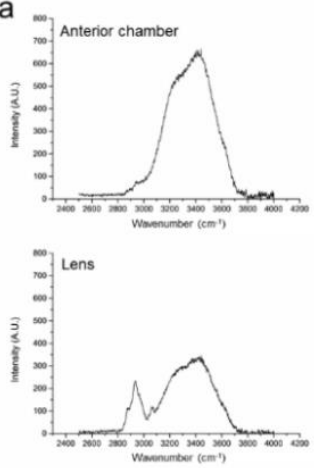
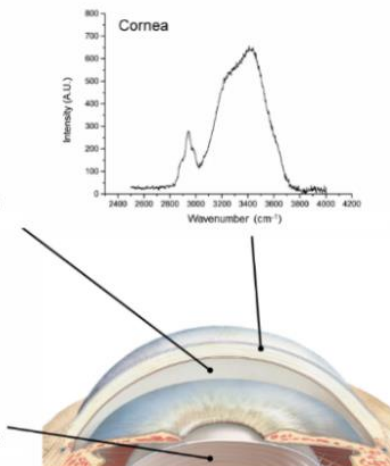

b

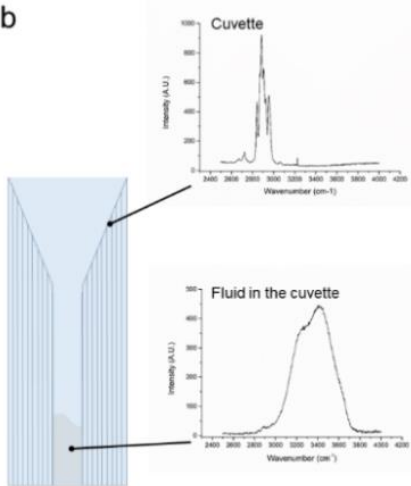

Figure 5. Location determination using high wave number measurement (671 nm Laser). (a) In the eye, and in (b) a cuvette. 

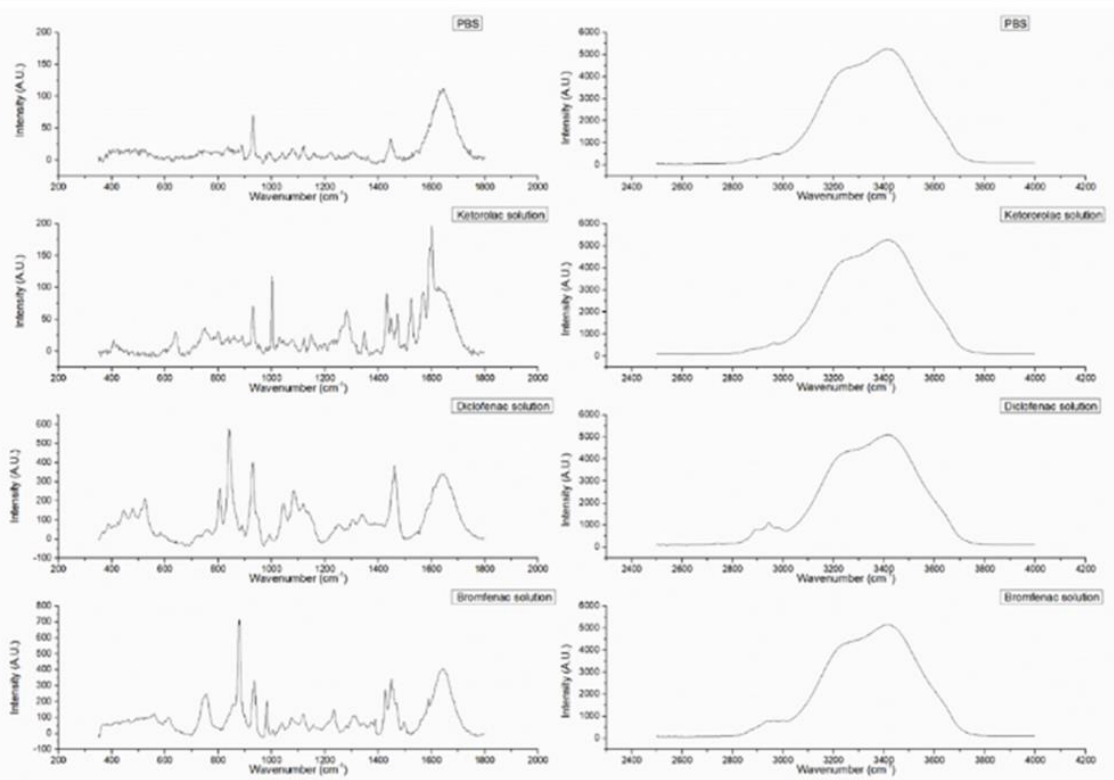<smiles>O=C(c1ccccc1)c1ccc2n1CCC2C(=O)O</smiles>

Ketorolac<smiles>Nc1c(CC(=O)O)cccc1C(=O)c1ccc(Br)cc1</smiles>

Bromfenac<smiles>O=C(O)Cc1ccccc1Nc1c(Cl)cccc1Cl</smiles>

Diclofenac

Figure 6. Raman spectra providing fingerprint signal (left column) and a high wave number signal (right column) of PBS and three different drugs (ketorolac (Acular®), Bromfenac (Yellox®), and Diclofenac (Naclof ()$)$ in ophthalmic solution. With corresponding molecular structure.

\section{Data processing}

Removal of cosmic ray spikes

All Raman spectra were loaded into OriginPro 9.0.0 (64 bit ed. OriginLab corp. Northampton, US) and were one-by-one checked (manually) for cosmic ray spikes. The wavenumbers affected by cosmic ray spikes were replaced by the values of the same wavenumbers from another frame. When this was done, the files were saved and loaded into MatLab $^{\odot}$ (Version 2017b, The Mathworks Inc., Natick, MA, US) for further processing.

Averaging of the frames, and removal of background noise and instrumental noise

The following process is programmed in the MatLab ${ }^{\odot}$ file ("Polynomial_Tophat background_subtraction_methods.m”), provided with the manuscript. 
First, frames were averaged to reduce fluctuations. Because the baseline has a strong influence on the polynomial approximation, the polynomial degree must be selected according to the shape of the baseline. In our system, using eyes, a $5^{\text {th }}$ degree polynomial fitting resulted in the most optimal background correction (figure S1). Therefore, we applied partial $5^{\text {th }}$ degree polynomial fitting with the morphology approach of Perez-Pueyo et al. ${ }^{12}$ to remove instrumental noise. First, all spectra were dissected in different zones, 350 $\mathrm{cm}^{-1}$ to $450 \mathrm{~cm}^{-1}, 450 \mathrm{~cm}^{-1}$ to $750 \mathrm{~cm}^{-1}, 750 \mathrm{~cm}^{-1}$ to $1250 \mathrm{~cm}^{-1}, 1250 \mathrm{~cm}^{-1}$ to $1650 \mathrm{~cm}^{-1}$, and $1650 \mathrm{~cm}^{-1}$ to $1800 \mathrm{~cm}^{-1}$. Zones that only contain fluorescence $\left(400 \mathrm{~cm}^{-1}\right.$ to $450 \mathrm{~cm}^{-1}, 800$ $\mathrm{cm}^{-1}$ to $1200 \mathrm{~cm}^{-1}$, and $1600 \mathrm{~cm}^{-1}$ to $1800 \mathrm{~cm}^{-1}$ ) (figure 7, zone 1, 2, and 3) are used calculate the polynomial function coefficients. The zone containing the water-peak (1550 $\mathrm{cm}^{-1}$ to $1650 \mathrm{~cm}^{-1}$ ) was excluded from the polynomial function fitting calculation. The achieved $5^{\text {th }}$ degree polynomial function was applied on the full spectrum $\left(400 \mathrm{~cm}^{-1}\right.$ to 1700 $\mathrm{cm}^{-1}$ ) to remove the fluorescence background (figure 7). Hereafter, the morphology-based Tophat method from Perez-Pueyo et al. ${ }^{12}$ was applied to eliminate instrumental noise. Examples of processed Raman signals are shown in figure 8.

Figure 9 shows the effect of data processing using the MatLab ${ }^{\circ}$ program on a sample without (figure 9a) and with (figure 9b) instrumental noise. In both occasions, a flat baseline is observed, and in figure $9 \mathrm{~b}$ instrumental noise is reduced without affecting the peaks. A full overview of the corrected data can be found in Bertens et al. ${ }^{6}$, and the full data-set is available supplementary to the manuscript from Zhang et al. ${ }^{7}$
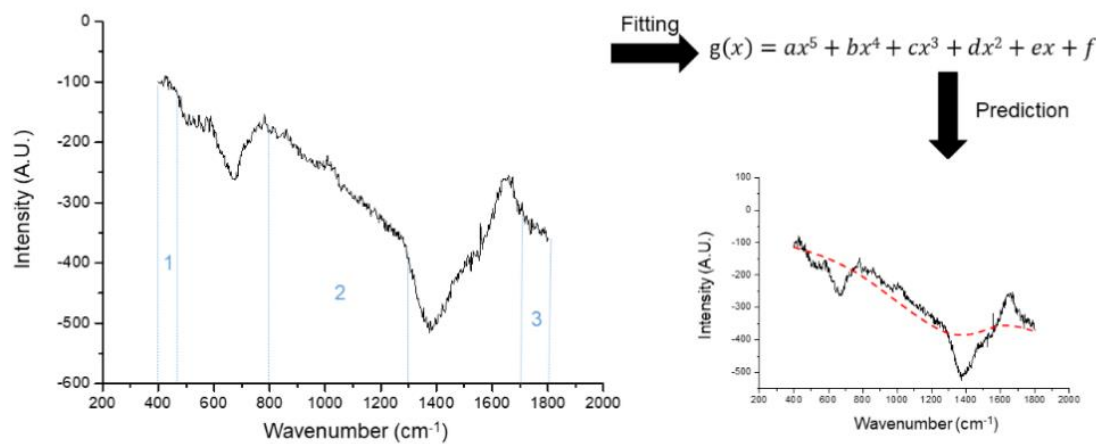

Figure 7. Overview of partial polynomial fitting. The spectrum is divided into different zones $(1,2$, and 3$)$, where after, a line was fitted through those zones based on a $5^{\text {th }}$ order polynomial function. The predicted line was withdrawn from the graph. 

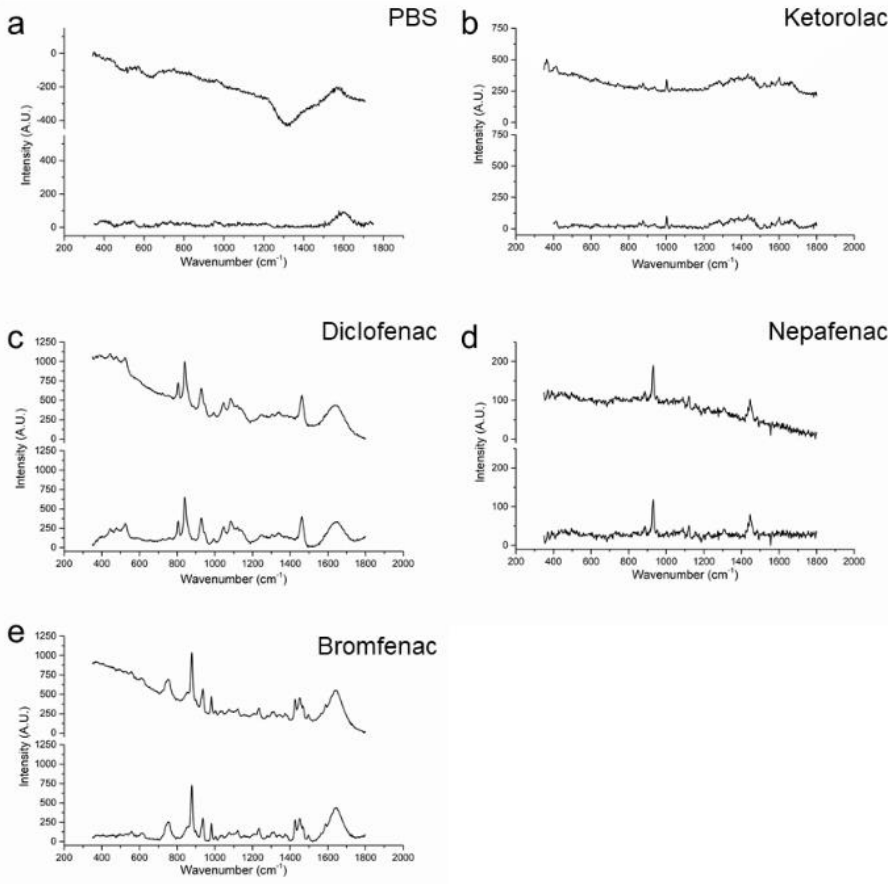

Figure 8. Effect of the processing on the data. (a) PBS in a rabbit eye, (b) ketorolac in a porcine eye, (c) diclofenac in a porcine eye, (d) nepafenac in a porcine eye, and (e) bromfenac in a porcine eye. The upper line shows RAW Raman signal and the lower line represents a processed Raman signal. Exposure time 30s, average of 3 frames.

a

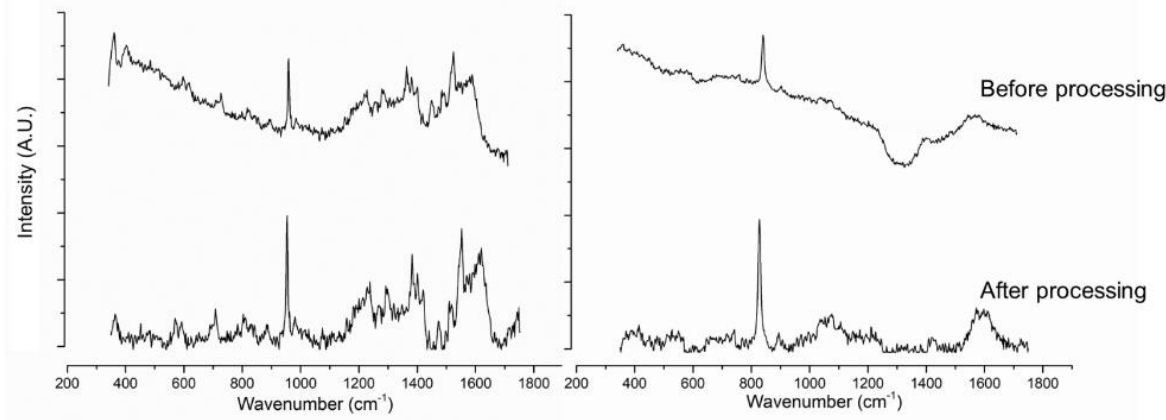

Figure 9. Effect of the processing on instrumental influences. (a) Shows a graph without instrumental influences (PBS) and (b) shows a graph with instrumental influences (rabbit eye). The upper line shows a RAW Raman spectrum and the lower line represents a processed Raman spectrum. Sample (a) is an ex vivo porcine eye, measured with Gonio lens, treated with $1.25 \%$ ketorolac tromethamine (ophthalmic solution), exposure time 60s, 3 frames. Sample (b) is an in vivo measurement of a rabbit eye (non-treated), measured with Gonio lens, exposure time 30s, 2 frames. 


\section{References}

1. D. A. Long, "Handbook of Raman spectroscopy. From the research laboratory to the process line," in Journal of Raman Spectroscopy I. R. L. a. H. G. M. Edwards., Ed., pp. 91-91, Marcel Dekker, New York and Basel (2004).

2. K. A. Esmonde-White et al., "Raman spectroscopy as a process analytical technology for pharmaceutical manufacturing and bioprocessing," Analytical and Bioanalytical Chemistry 409(3), 637-649 (2017).

3. K. Buckley, and A. G. Ryder, "Applications of Raman Spectroscopy in Biopharmaceutical Manufacturing: A Short Review," Appl Spectrosc 71(6), 1085-1116 (2017).

4. K. Hosseini et al., "Noninvasive detection of ganciclovir in ocular tissue by Raman spectroscopy: implication for monitoring of drug release," J Ocul Pharmacol Ther 18(3), 277-285 (2002).

5. H. J. Byrne et al., "Spectral pre and post processing for infrared and Raman spectroscopy of biological tissues and cells," Chem Soc Rev 45(7), 1865-1878 (2016).

6. C. J. F. Bertens et al., "Confocal Raman spectroscopy: Evaluation of a non-invasive technique for the detection of topically applied ketorolac tromethamine in vitro and in vivo," Int J Pharm 570(118641 (2019).

7. S. Zhang et al., "In vitro and in vivo datasets of topically applied ketorolac tromethamine in aqueous humor using Raman spectroscopy," Data Brief 27(104694 (2019).

8. R. E. Littleford et al., "Raman spectroscopy of street samples of cocaine obtained using Kerr gated fluorescence rejection," Analyst 129(6), 505-506 (2004).

9. D. V. Martyshkin et al., "Effective suppression of fluorescence light in Raman measurements using ultrafast time gated charge coupled device camera," Review of Scientific Instruments 75(3), 630-635 (2004).

10. J. Zhao et al., "Automated autofluorescence background subtraction algorithm for biomedical Raman spectroscopy," Appl Spectrosc 61(11), 1225-1232 (2007).

11. X. Zhang et al., "Method for Removing Spectral Contaminants to Improve Analysis of Raman Imaging Data," Sci Rep 7(39891 (2017).

12. R. Perez-Pueyo, M. J. Soneira, and S. Ruiz-Moreno, "Morphology-based automated baseline removal for Raman spectra of artistic pigments," Appl Spectrosc 64(6), 595-600 (2010). 


\section{Supplementary material}

Matlab $^{\odot}$ script: "Polynomial_Tophat_background_subtraction_methods.m". This script can be downloaded from the journal's website.
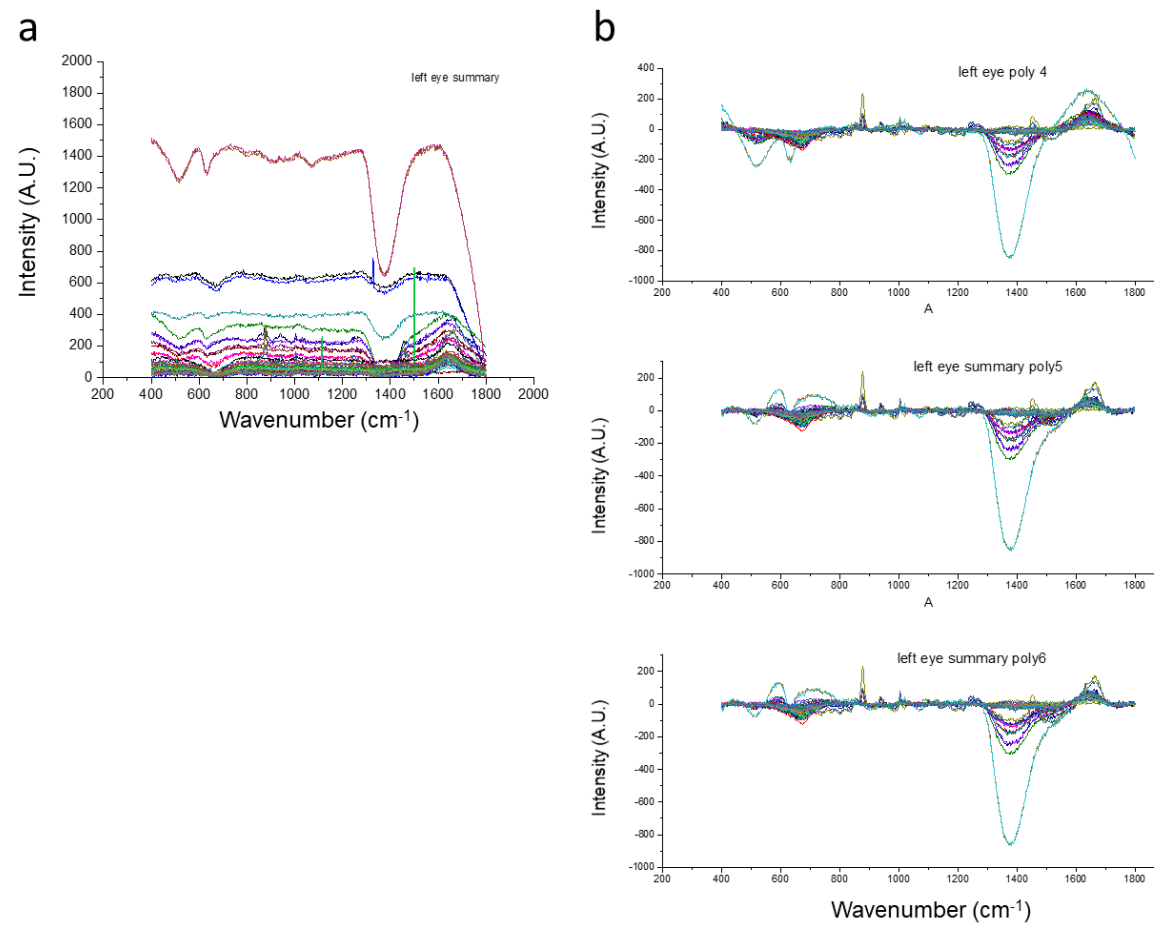

Figure S1. Optimization of the polynomial fitting. (a) RAW data before polynomial correction. (b) Data corrected using a $4^{\text {th }}$, a $5^{\text {th }}$, and a $6^{\text {th }}$ polynomial fitting function as shown in figure 5 . No difference between the $5^{\text {th }}$ and $6^{\text {th }}$ polynomial fitting was observed; therefore, $5^{\text {th }}$ order polynomial fitting was used during the corrections. 
Page | 78 


\section{Chapter 4b}

\section{In vitro and in vivo datasets of topically applied ketorolac tromethamine in aqueous humor using Raman spectroscopy}

Shuo Zhang", Christian J.F". Bertens, Roel J. Erckens, Frank J.H.M. van den Biggelaar, Tos T.J.M. Berendschot, Carroll A.B. Webers, Rudy M.M.A. Nuijts and Marlies Gijs \# Equal Contribution Data in Brief 2019;27:104694 DOI: 10.1016/j.dib.2019.104694 


\begin{abstract}
This article includes datasets acquired by Raman spectroscopy from in vivo and in vitro ocular samples collected from the dataset from Bertens et al., "Confocal Raman spectroscopy: Evaluation of a non-invasive technique for the detection of topically applied ketorolac tromethamine in vitro and in vivo" . Detection of ketorolac tromethamine in pig eyes was performed in vitro and rabbit eyes in vivo. Extracted aqueous humor samples from pig and rabbit eyes were measured in vitro using a cuvette. This manuscript shows the spectral Raman data without pre-treatment or analysis from ocular tissues and provides further information towards aqueous humor research via alternative data processing methods. Furthermore, the raw data enclosed may be used for future aqueous humor investigations and pharmaceutical research.
\end{abstract}

\title{
Highlights
}

- The dataset could be used for further composition analysis of the aqueous humor and for future pharmaceutical research, to increase sensitivity of Raman systems.

- The dataset can be useful for researchers who are interested in the aqueous humor composition, ocular pharmaceutics, Raman spectroscopy, and software engineers.

- Alternative processing methods could be applied to exact other compounds in the aqueous humor or to enhance signals.

- This dataset offers a large cohort of animals measured on both eyes, 5 times. 


\section{Data}

The data contains unanalysed Raman spectra obtained from pig eyes (in vitro) (6b.1.1, see supplementary files folder 'in vitro pig eyes' and 'in vitro cuvettes, aqueous humor from pig eyes'), rabbit eyes (in vivo) (6b.1.2, see supplementary files folder 'in vivo rabbit eyes'), and aqueous humor samples (in vitro, see supplementary files folder 'in vitro cuvettes, aqueous humor from rabbit eyes') (6b.1.3). Based on the differences of the samples, three types of set-ups were used on each dataset. For pig eye measurements in vitro, a longworking-distance microscope objective lens (Jena lens alone or a Gonio lens combined with a f60 lens) was utilized (see supplementary files 'in vitro pig eyes' folder 'jena lens' or 'gonio'). For the rabbit eyes measurements in vivo, a Gonio lens combine with a f60 lens was used. For cuvettes measurements, a 800 lens was used when the sample was measured in a Brand ${ }^{\circledR}$ cuvette. $^{2}$ For each experimental set-up, the fingerprint-wavenumber region (patterns specific for a drug-molecule, ranging from $350 \mathrm{~cm}^{-1}$ to $1800 \mathrm{~cm}^{-1}$ ) and the highwavenumber region (higher energy shifted, ranging from $2500 \mathrm{~cm}^{-1}$ to $4000 \mathrm{~cm}^{-1}$ ) were included. The fingerprint spectra dataset was used for detection of intraocular ketorolac tromethamine as described in the article of Bertens et al. ${ }^{1}$ Several peaks could be identified in the fingerprint region spectrum of a ketorolac tromethamine sample (figure 1a). Only major peaks specific for ketorolac tromethamine were selected. Those peaks are assigned to certain chemical bonds or vibration modes. The assignment of the ketorolac related peaks is presented in Table $1 .{ }^{1}$ Due to the spectrometer's spectral resolution $\left(2 \mathrm{~cm}^{-1}\right)$, the peak observed at $1586 \mathrm{~cm}^{-1}$ is assigned to $\mathrm{NH}_{2}$ deformation. ${ }^{3}$ The peak of $1524 \mathrm{~cm}^{-1}$ is assigned to in-plane vibrations of the conjugated $-\mathrm{C}=\mathrm{C}$-. The observed peak at $1472 \mathrm{~cm}^{-1}$ is assigned to $\mathrm{C}=\mathrm{N}$ stretching and the peak at $1282 \mathrm{~cm}^{-1}$ is assigned to $\mathrm{CH}_{2}$ wagging vibrations. Because Raman spectrum of the cornea, aqueous humor, and lens show different patterns in the high-wavenumber region, spectra from this region could be used as guide for location determination in the ocular tissue (figure $1 b){ }^{4-6}$
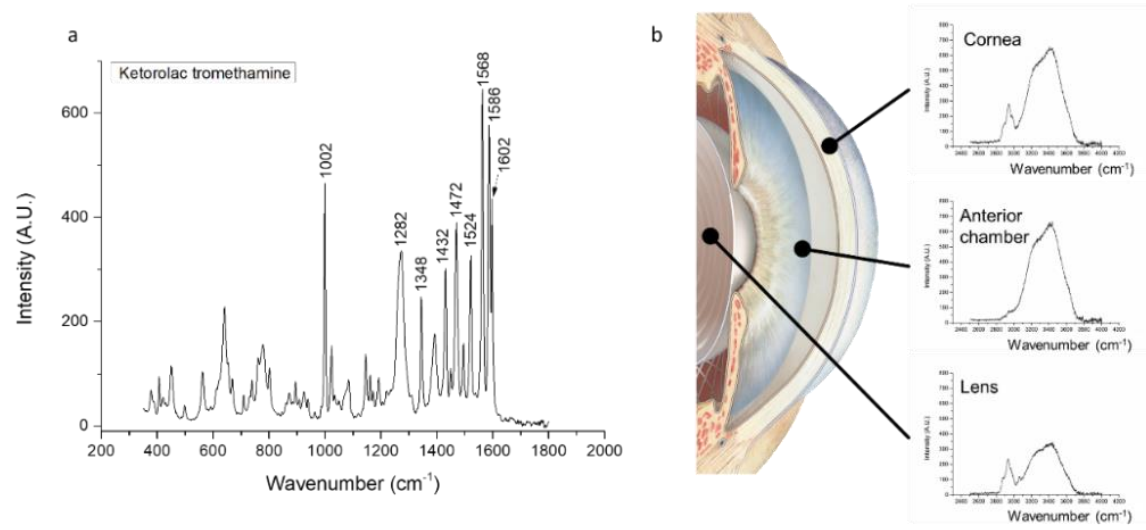

Figure 1. (a) Fingerprint spectra of Ketorolac powder. (b) Determination of the location in the eye using highwavenumber Raman spectra. Spectra are from pig eyes, 3 frames of 10 seconds averaged measured using a Jena 
lens.

Table 1. Main characteristic bands assignment of ketorolac ${ }^{7}$

\begin{tabular}{|l|l|l|}
\hline \multicolumn{1}{l}{$\begin{array}{l}\text { Peak location } \\
\text { (wavenumber) }\end{array}$} & Intensity & Peak Assignment \\
\hline $1002 \mathrm{~cm}^{-1}$ & very strong & Phenylalanine or a C-C aromatic ring stretching \\
\hline $1282 \mathrm{~cm}^{-1}$ & medium & $\mathrm{CH}_{2}$ wagging vibrations \\
\hline $1348 \mathrm{~cm}^{-1}$ & weak & An unassigned mode \\
\hline $1432 \mathrm{~cm}^{-1}$ & strong & $\mathrm{CH}$ bond \\
\hline $1472 \mathrm{~cm}^{-1}$ & medium & $\mathrm{C}=\mathrm{N}$ stretching \\
\hline $1524 \mathrm{~cm}^{-1}$ & medium & In-plane vibrations of the conjugated $-\mathrm{C}=\mathrm{C}-$ \\
\hline $1568 \mathrm{~cm}^{-1}$ & very strong & $\mathrm{COO}-$ \\
\hline $1586 \mathrm{~cm}^{-1}$ & strong & $\mathrm{NH}$ deformation \\
\hline $1602 \mathrm{~cm}^{-1}$ & medium & Phenylalanine or a $\mathrm{C}==\mathrm{C}$ bond. \\
\hline
\end{tabular}

\section{In vitro, dataset}

Pig eyes (enucleated) were immersed in the dark at $4{ }^{\circ} \mathrm{C}$ for 24 hours in vitro in different concentrations of ketorolac solutions $(0.05 \%$ to $5.0 \%)$ before the measurements (see supplementary files folder 'in vitro pig eyes'). For each concentration, three eyes were measured by Raman spectroscopy. An example spectrum obtained from a pig eye is shown in figure 2. The location in the eye was determined using the high-wavenumber spectra (figure 2b).

a

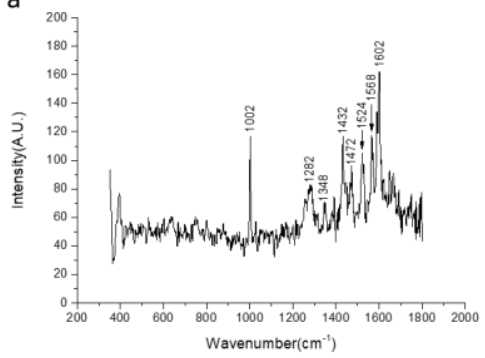

b

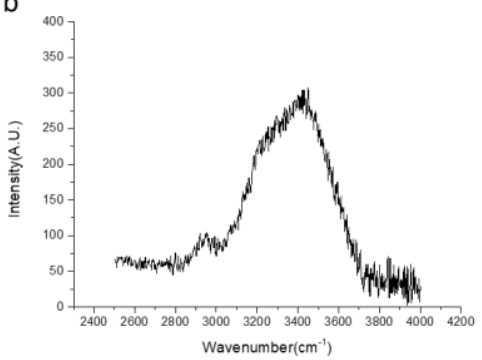

Figure 2. Raman spectrum of a pig eye soaked in a 5\% ketorolac solution obtained by Jena lens. (a) Fingerprint spectrum, obtained using 60 seconds and averaged for 3 frames. (b) High-wavenumber spectrum, obtained using 60 seconds and averaged for 3 frames. No correction has been applied on the spectra. 


\section{In vivo dataset}

New Zealand white rabbits received $50 \mu \mathrm{L}$ Acular $^{\circledR}$ three times a day in their right eye. At the same time, they received a drop of buffered saline solution (BSS) in their left eye as a control (see supplementary files folder 'in vivo rabbit eyes'). The measurement parameters of the Raman system were optimized using the first four rabbits. Different integration times $(10,15$, or 30 seconds) were measured to acquire the optimum Raman signal. The following measurements were performed using an integration time of 30 seconds. During these measurements, hardware influences were observed. Further optimization of the processing method can be seen in Bertens et al. ${ }^{2}$ The difference of the variant integration times can be found in figure 3, for example, the spectrum intensity at $400 \mathrm{~cm}^{-1}$ is from 74 A.U. with 10 second integration time (figure 3a), 127 A.U. with 15 second integration time (figure $3 b$ ) and 333 A.U. with 30 second integration time (figure 3c). Rabbits were measured according to the schedule in Table 2.
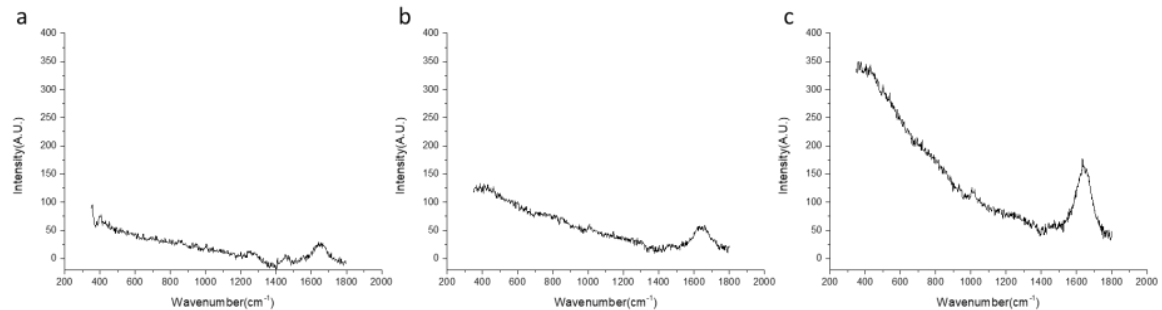

Figure 3. In vivo Raman spectrum of the right eye of a rabbit with different integration times, averaged for 2 frames. (a) Shows the graph for 10 seconds, (b) 15 seconds, and (c) 30 seconds. 
Table 2. In vivo integration time of the Raman measurements of the rabbits

\begin{tabular}{|c|c|c|c|c|c|c|}
\hline No. & Name & Day 0 & Day 7 & Day 14 & Day 21 & Day 28 \\
\hline 1 & PLAC & $x$ & $10 \mathrm{~s}$ & $15 \mathrm{~s}$ & $15 s$ & $30 \mathrm{~s}$ \\
\hline 2 & PLBT & $10 \mathrm{~s}$ & $x$ & $15 \mathrm{~s}$ & $15 \mathrm{~s}$ & $30 \mathrm{~s}$ \\
\hline 3 & PKXF & $10 \mathrm{~s}$ & $10 \mathrm{~s}$ & $15 s$ & $15 s$ & $30 \mathrm{~s}$ \\
\hline 4 & PKYJ & $10 \mathrm{~s}$ & $10 \mathrm{~s}$ & $15 \mathrm{~s}$ & $15 \mathrm{~s}$ & 30s \\
\hline 5 & PNRS & $30 \mathrm{~s}$ & $30 \mathrm{~s}$ & $30 \mathrm{~s}$ & $30 s$ & $30 \mathrm{~s}$ \\
\hline 6 & PNPH & $30 \mathrm{~s}$ & $x$ & & & \\
\hline 7 & PNPJ & $30 \mathrm{~s}$ & $30 \mathrm{~s}$ & $30 \mathrm{~s}$ & $30 s$ & $30 \mathrm{~s}$ \\
\hline 8 & PNLJ & $30 \mathrm{~s}$ & $30 \mathrm{~s}$ & $30 \mathrm{~s}$ & $30 \mathrm{~s}$ & $30 \mathrm{~s}$ \\
\hline 9 & POLI & $30 \mathrm{~s}$ & $x$ & & & \\
\hline 10 & POBS & $30 \mathrm{~s}$ & $30 \mathrm{~s}$ & $30 \mathrm{~s}$ & $30 \mathrm{~s}$ & $30 \mathrm{~s}$ \\
\hline 11 & PPDI & $30 \mathrm{~s}$ & $30 \mathrm{~s}$ & $30 \mathrm{~s}$ & $30 s$ & $30 s$ \\
\hline 12 & $\mathrm{POHI}$ & $30 \mathrm{~s}$ & $30 \mathrm{~s}$ & $30 \mathrm{~s}$ & $30 s$ & $30 \mathrm{~s}$ \\
\hline
\end{tabular}

Integration time is shown in seconds, ' $x$ ' represents a failed measurement or no data. 2 frames per measurement were used.

\section{In vitro, cuvettes dataset}

Immediately after intra-ocular Raman measurements (both in vitro \& in vivo), $100 \mu \mathrm{L}$ to $150 \mu \mathrm{L}$ of aqueous humor was drawn from the pig eyes, and $50 \mu \mathrm{L}$ was drawn from the right eye of each rabbit. The aqueous humor samples were frozen on dry ice and stored in a $-80^{\circ} \mathrm{C}$ freezer until use. When used, the location of focus was determined with the high wavenumber spectra, as shown in figure 4.

Fingerprint spectra were collected to determine ketorolac concentrations in the aqueous humor. Spectrum examples of pig and rabbit aqueous humor are show in figure $5 a$ and figure 5b, respectively (see supplementary files folder 'in vitro cuvettes'). Further background subtraction needs to be applied for analyses. 


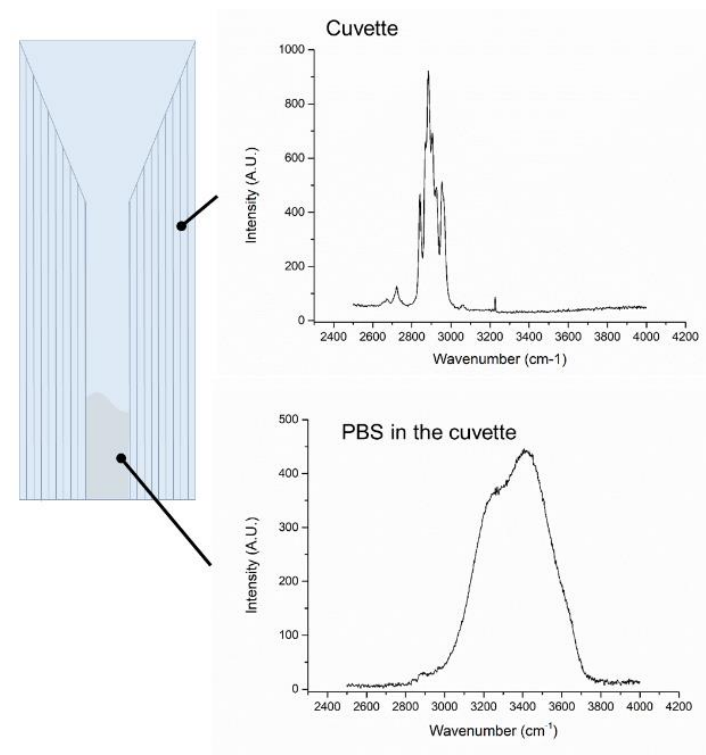

Figure 4. Laser focus positioning in a cuvette filled with PBS (pH7.4) using the high wavenumber spectrum.
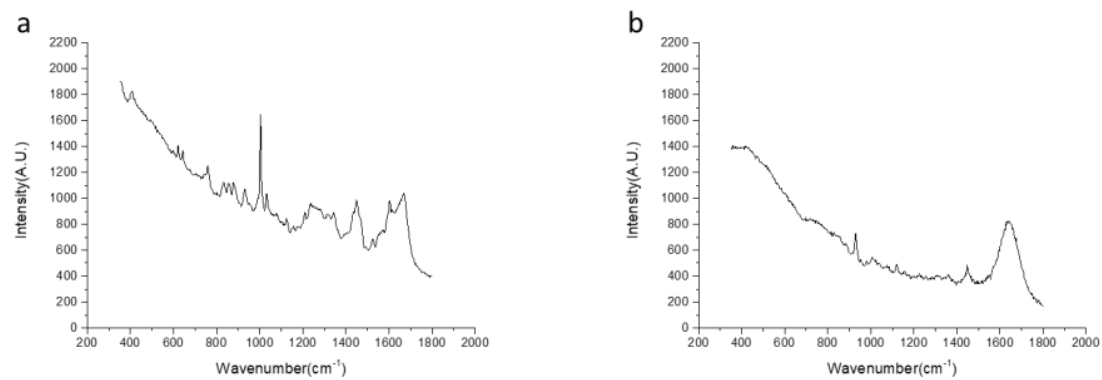

Figure 5. Raman spectrum of aqueous humor samples from, (a) a $0.5 \%$ ketorolac submerged pig eye (3 frames of 60 seconds), and (b) from a rabbit eye ( 3 frames of 60 seconds on PKXF samples).

\section{Experimental design, materials, and methods}

\section{Raman spectroscopy system}

Two diode lasers were utilized as an excitation light source for Raman spectroscopy: a 26mW 785nm laser (Innovative Photonic Solutions SM 785 nm, Monmouth Junction, NJ, US) or a $14 \mathrm{~mW} 671 \mathrm{~nm}$ laser (Laser Quantum Ignis 671 and SMD 6000, Konstanz, DE). A high-performance Raman spectrometer module (model 2500, River Diagnostics ${ }^{\circledR}$, Rotterdam, NL) was utilized for Raman spectra recordings. ${ }^{8}$ A $25 \mu \mathrm{m}$ diameter pinhole was integrated within the spectrometer for the confocal Raman spectroscopy detection. An aircooled charge-coupled device (CCD) camera with operating temperature $-60^{\circ} \mathrm{C}$ was 
integrated within the spectrometer for signal detection. The Raman spectrometer is capable of collecting Raman scattering wavenumber ranges in $350 \mathrm{~cm}^{-1}-1800 \mathrm{~cm}^{-1}$ and $2500 \mathrm{~cm}^{-1}$ $4000 \mathrm{~cm}^{-1}$ with $2 \mathrm{~cm}^{-1}$ spectral resolution. A diverged laser beam out of the spectrometer is converted to a collimation beam by a lens with focus length of $80 \mathrm{~mm}$ (f80). Depending on the measurement, the lens setup was adapted. The system was used in single point modus and location in the sample was determined using the high wave numbers (671 nm laser).

\section{In vitro measurement of enucleated pig eyes}

Fresh domestic pig (Sus Scrofa Domesticus) eyes were obtained from a local abattoir ("Slachthuis Kerkrade Holding", Kerkrade, NL). The enucleated eyes were transported to the laboratory on ice and used within 3 hours after enucleation. Before use, the pig eyes were inspected with a stereo microscope (Olympus SZX9, Tokyo, JP). Only eyes with clear corneas without visible corneal damage were used in the experiment. The excess tissues of the eye were removed carefully where after the eyes were washed in phosphate buffered saline (PBS) ( $\mathrm{pH}$ of 7.4). Meanwhile, ketorolac (MSN laboratories, Telangana, IN) was dissolved in PBS creating concentrations of $0.05 \%, 0.1 \%, 0.125 \%, 0.25 \%, 0.5 \%, 1.0 \%$, $1.25 \%, 2.5 \%$, and $5.0 \%$. The pig eyes were submerged in $15 \mathrm{~mL}$ of a diluted ketorolac solution. As negative control, PBS was used, and as positive control $0.5 \%$ ketorolac ophthalmic solution (Acular ${ }^{\mathrm{TM}}$, Allergan, Dublin, IR) was used as submerging solution. For each concentration, three eyes were used. Before the Raman measurements, pig eyes were stored in the dark at $4^{\circ} \mathrm{C}$ for 24 hours. Before measurements were taken, the eyes were inserted in a home-designed holder (figure 6).

A long-working-distance microscope objective lens (Jena lens, magnification x 25; numerical aperture $=0.50$; focal length $=10 \mathrm{~mm}$; Carl Zeiss, Jena, DE) was used as focus lens for the Raman system (figure 7a). A f60 lens combined with a Gonio lens (Haag-Streit Meridian, CGA1, Köniz, CH) also been used for pig eye measurement (figure $7 b$ ). Methocel $^{\circledR} 2 \%$ (OmniVision ,Santa Clara, CA, US) was used to connect the Gonio lens to the cornea. The samples were exposed to 3 frames for 60 seconds. A detailed description can be found in the manuscript from Bertens et al. ${ }^{1}$
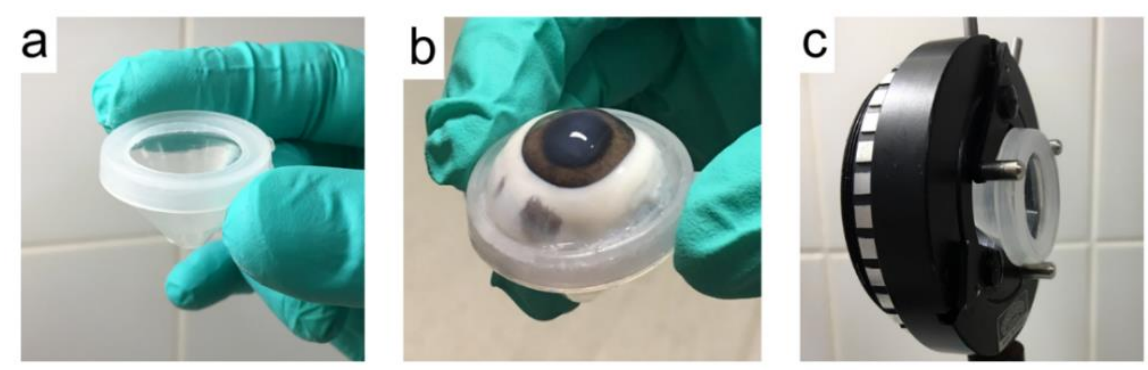

Figure 6. Holder for enucleated eyes. (a) shows an empty holder, (b) shows a holder with a pig eye, and (c) shows the empty holder on an adjustable lens mount. 


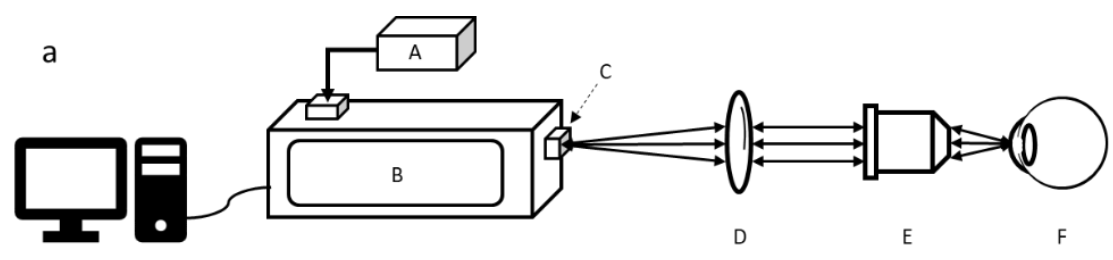

G

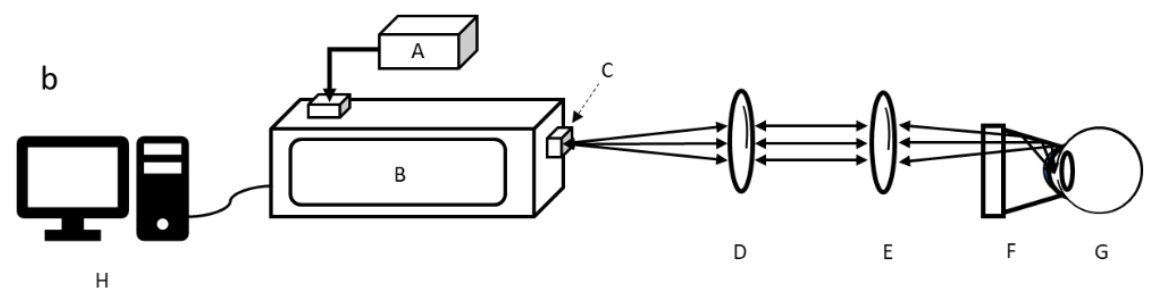

Figure 7. In vitro and in vivo settings of the Raman system.

(a) The set-up is for in vitro pig eye measurements by a Jena lens. (A) laser; (B) Raman module, with (C) $25 \mu \mathrm{m}$ pinhole; (D) collimation 880 lens; (E) objective (Jena lens); (F) pig eye; (G) computer.

(b) The set-up is for in vitro pig eye and in vivo rabbit measurements, a Gonio lens in combination with a f60 focus lens are used for focus in the anterior chamber of the animal eye. (A) laser; (B) Raman module, with (C) $25 \mu \mathrm{m}$ pinhole; (D) collimation f80 lens; (E) f60 lens; (F) a Gonio (one-mirror) lens; (G) pig eye (in vitro) or rabbit eye (in vivo); $(\mathrm{H})$ computer.

Arrows indicate direction of excitation laser light and backscattered Raman light.

\section{In vivo measurement of the rabbit eyes}

Twelve New Zealand white rabbits (weight ranged from $2.0 \mathrm{~kg}$ to $2.5 \mathrm{~kg}$ upon arrival) were obtained from Envigo (Horst, NL). The rabbits were group housed with 6 animals per cage with males and females separated. The rabbits had ad libitum access to water and food. One week was given to acclimatize before rabbits were used in the experiments. The rabbits were treated with $50 \mu \mathrm{L}$ Acular ${ }^{\mathrm{TM}}$ in the lower conjunctival fornix of their right eye. The contralateral eyes were treated with $50 \mu \mathrm{L}$ sterile buffered saline solution (BSS, B. Braun, Melsungen AG, DE manufacturer). Both treatments were performed three times a day. Measurements were taken on day 0 , day 7 , day 14 , day 21 , and day 28 . Four rabbits were used to optimize the system parameters as shown in Table 2.

Rabbits were measured using setup as shown in figure $7 b$. During the examinations, rabbits were anesthetized intramuscularly with ketamine (Alfasan, Woerden, NL) and midazolam (Actavis, Dublin, IR), $50 \mathrm{mg} / \mathrm{kg}$ and $5 \mathrm{mg} / \mathrm{kg}$, respectively. Both eyes of the rabbit were measured by the Raman system. All measurements were performed at random, 1 to 3 hours after receiving the eye drops. Measurement was performed with 30 second exposure times using 2 frames. All animal procedures were conducted according to the ARVO Statement for the Use of Animals in Ophthalmic and Visual Research and the Guidelines of the Central Laboratory Animal Facility of Maastricht University. All 
protocols were approved by the Central Committee for Animal research and were in accordance with the European Guidelines (2010/63/EU).

\section{In vitro measurement of the aqueous humor}

For cuvette detection, $50 \mu \mathrm{L}$ to $150 \mu \mathrm{L}$ aqueous humor was obtained from an anterior chamber paracentesis from the eyes using an insulin syringe (BD Micro-Fine ${ }^{\mathrm{TM}}$, Becton Dickinson, NJ, US). 50uL was drawn from rabbit eyes after topical sedation (1 drop 0.4\% Oxybuprocaine hydrochloride solution (Bausch \& Lomb Pharma, Brussels, BE)), $100 \mu \mathrm{L}$ to $150 \mu \mathrm{L}$ was drawn from the pig eyes. As a negative control, $100 \mu \mathrm{L}$ aqueous humor was drawn from seven healthy control rabbits within 10 minutes after sacrifice, no topical treatment nor anaesthetics were used.

All aqueous humor samples were frozen on dry ice immediately after sampling and stored in a $-80^{\circ} \mathrm{C}$ freezer until measurements. Samples were measured using a $\mathrm{f} 80$ lens in front of the sample container (figure 8). The sample was measured for 3 frames in a disposable cuvette (\#7592-00, Sigma-Aldrich, MO, US) with 60 seconds per frame.

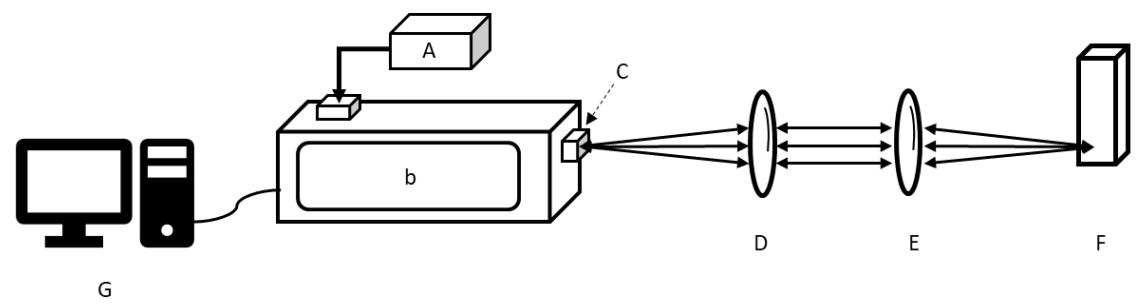

Figure 8. In vitro settings of the Raman system with a cuvette. (A) laser; (B) Raman module, with (C) $25 \mu \mathrm{m}$ pinhole; (D) collimation f80 lens; (E) focusing f80 lens (F) samples within cuvette; (G) computer. Arrows indicate direction of excitation laser light and backscattered Raman light. 


\section{References}

1. C. J. F. Bertens et al., "Confocal Raman spectroscopy: Evaluation of a non-invasive technique for the detection of topically applied ketorolac tromethamine in vitro and in vivo," Int J Pharm 570(118641 (2019).

2. C. J. F. Bertens* et al., "Pipeline for the removal of hardware related artifacts and background noise for Raman spectroscopy," MethodsX (2019).

3. R. Treffer et al., "Distinction of nucleobases - a tip-enhanced Raman approach," Beilstein $J$ Nanotechnol 2(628-637 (2011).

4. R. J. Erckens et al., "Raman spectroscopy in ophthalmology: from experimental tool to applications in vivo," Lasers Med Sci 16(4), 236-252 (2001).

5. R. J. Erckens et al., "Drug-induced corneal hydration changes monitored in vivo by non-invasive confocal Raman spectroscopy," Journal of Raman Spectroscopy 32(9), 733-737 (2001).

6. $\quad$ A. C. Bot et al., "Raman microspectroscopy of fixed rabbit and human lenses and lens slices: new potentialities," Exp Eye Res 49(2), 161-169 (1989).

7. Z. Movasaghi, S. Rehman, and I. U. Rehman, "Raman spectroscopy of biological tissues," Applied Spectroscopy Reviews 42(5), 493-541 (2007).

8. M. Elshout et al., "Detection of Raman spectra in ocular drugs for potential in vivo application of Raman spectroscopy," J Ocul Pharmacol Ther 27(5), 445-451 (2011). 
Page | 90 


\section{Chapter 5}

Dark field illumination in conjunction with confocal Raman spectroscopy for real time non-invasive aqueous humor investigation

Shuo Zhang, Roel J. Erckens, Frans H.M. Jongsma, John de Brabander, Carroll A.B. Webers and Tos T.J.M. Berendschot

Optical Engineering 2020;59:092002 DOI: $10.1117 / 1.0 e .59 .9 .092002$ 


\begin{abstract}
To obtain a real-time noninvasive analysis of the content of the aqueous humor (AH) in the eye, a confocal dark field ophthalmic probe was designed and optimized by raytracing software. The confocal technique allows Raman spectrometric sampling of the AH with suppressing signals from the surrounding tissues. Dark field illumination prevents the excitation light to reach the vulnerable retinal tissue directly. In order to evaluate the optical performance and safety of this method, a functional prototyped eye contact probe has been designed and tested on rabbit ex vivo eyes.
\end{abstract}

\title{
Keywords
}

Raman spectroscopy, Eye; Aqueous Humor, Confocal microscopy, Dark field illumination 


\section{Introduction}

The anterior chamber (AC) (Figure 1) contains an ocular fluid called aqueous humor $(\mathrm{AH})$ which functions are maintaining eye pressure and provide nutrition to the cornea and lens. If these proteins, nucleotides and metabolites in the AH could be detected they could be helpful in understanding ocular diseases..$^{1-3}$ Glucose levels in AH correlate with those in blood plasma and may be used as an indicator of diabetes complications in the eye. ${ }^{4-6}$ Inflammatory cytokine profiles in the AH could reveal associations with glaucoma, the leading irreversible cause of blindness. ${ }^{7}$ Further, recently it was shown that three ocular proteins in the AH can be used as a diagnostic aid for several retinal diseases diagnoses. ${ }^{8}$ The current standard in clinical practice to analyze AH is invasive biopsy. However, this approach has several drawbacks: First, breaking the integrity of the eye raises the risk of inflammation. Secondly, it is a burden to the patients, which impairs its frequent use. Thirdly, the procedure requires multiple devices and a clinical environment. Fourthly, it also bears the risk of changing the composition of the $\mathrm{AH}$ during the process.

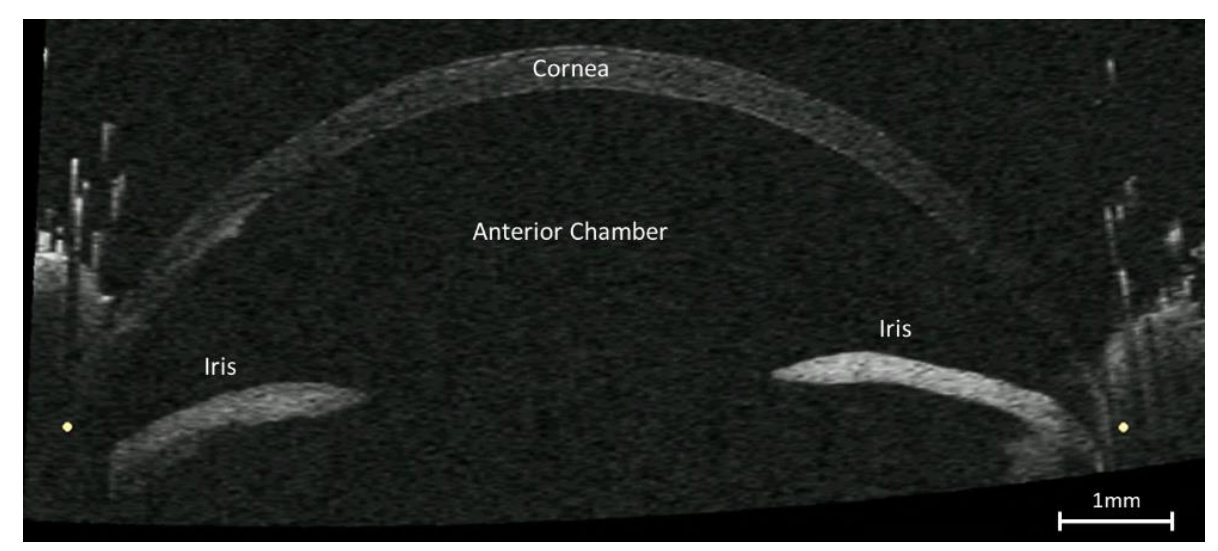

Fig. 1 OCT Image of anterior segment of a rabbit eye, acquired with a Heidelberg Engineering BD900 SL-OCT.

To understand the mechanism of eye diseases, it is preferably to investigate the physiological and pathological changes in real time of the eye with non-invasive methods. Since the eye is transparent in the electromagnetic spectrum of $350 \mathrm{~nm}$ to $2000 \mathrm{~nm}$, several optical diagnostic techniques have been developed for anterior eye section examinations, such as biomicroscopy (slit lamp) and Optical Coherence Tomography (OCT). ${ }^{9}$ However, these morphological examinations are lacking the ability of revealing the biochemical information of ocular tissues. Raman spectroscopy can do this in a non-invasive way. ${ }^{10}$ The Raman effect is associated with inelastic scattering from vibrations or rotations of chemical bonds and therefore the spectra have a specific fingerprint for each biomedical molecule due to their unique atomic composition and structure. As such Raman spectroscopy is capable to identify targeted tissues both qualitatively and quantitatively and is therefore 
widely used for molecular identification in biomedical and preclinical applications. ${ }^{2,11-14}$ To evaluate the feasibility of our Raman spectroscopy system for ocular research, the first Raman spectroscopy experiments of our research group to measure a rabbit eye were performed in 1990s at the UTMB in Galveston, Texas. ${ }^{15}$ With a set up using an $514.5 \mathrm{~nm}$ wavelength Argon ion laser as excitation source and a liquid Nitrogen cooled spectrometer, good isolated Raman spectra of the aqueous humor of an ex vivo rabbit eye were obtained as a proof of principle experiments. ${ }^{16}$

To obtain a high signal-to-noise ratio (SNR) in Raman spectroscopy a laser is indispensable as an excitation source. However, radiation limitations for light damage of retinal tissue are strict when employing laser light. ${ }^{16,17}$ There are two kinds of light hazard in the eye. Wavelengths below $550 \mathrm{~nm}$ might cause photochemical light damage on the retinal tissue. ${ }^{17}$ Here exposure time is limited due to its cumulative effect on living tissue. Longer wavelengths do not induce photochemical light damage and have the benefit of suppressing the disturbing background fluorescence. However, with longer wavelengths the danger of thermal damage increases while Raman responses decrease. For this reason, we chose excitation wavelengths at the end of the visual spectrum - that is patient friendly - of $671 \mathrm{~nm}$ and $785 \mathrm{~nm}$ at relative low intensities of 10 to $25 \mathrm{~mW}$ with prolonged exposure times of 60 to 90 seconds. Note that even this power excitation is unsafe when direct laser radiation reaches the retinal tissue. To prevent the latter and guarantee an eye safe usage, we designed and constructed a circular symmetric Raman eye probe for clinical use with the principle of 'dark field' illumination in which only scattered light can reach the patients retina. The optical performance of this probe will be presented in this paper using simulation diagrams. In addition, we will show results of ex vivo test in rabbit eyes using a prototype of the probe.

\section{Design}

To evaluate its optical principles, the basic structure of the probe was first designed and developed by Ray-tracing software Zemax (version 16.5 Sp3, Zemax LLC., Kirkland, USA). After performance optimization and validation of the design, the 3D model for manufacturing was generated by Autodesk inventor (professional version 2016, Autodesk corp., San Rafael, USA). In the end, the 3D model was imported into Zemax again for final check (like tolerance influence) before fabricating the prototype.

The spectrometer provides an excitation beam with a waist of about $18 \mu \mathrm{m}$. For simplicity, we assumed a point light source in ray-tracing for the optical setup. To optimize our dark-field design with Zemax, a Liou-Brennan eye model was used. ${ }^{18}$ All ray-tracings were done at $785 \mathrm{~nm}$.

\section{Optical design}

To prevent light damage on retinal tissue, direct excitation of the posterior part of the eye should be avoided. We have opted for the approach to apply dark field illumination 
principle for patients, with the incident laser light passing through the periphery of the cornea and focusing in the AC. This way it ends up in iris tissue instead of directly reaching the retina and only the scattered light can reach the posterior parts of the eye through the crystalline lens. It offers an integration length of about 0.5 millimeter (calculated for a 25 $\mu \mathrm{m}$ pinhole) perpendicular to the optical axis of the eye and the numerical aperture (NA) of the probe could be designed to match that of the Raman spectrometer (NA=0.05).

The probe was designed and developed based on a former patent (Figure 2a). ${ }^{19}$ Unfortunately, alignment of this model proved to be so difficult, that no reproducible results could be obtained. In particular, the distance between the focusing lens and the probe proved to be critical. In the redesigned probe a central mirror focuses the collimated incoming light that is further guided by two conical mirrors into the eye. This design eliminates the degree of freedom for the distance between separated focusing lens and the probe formerly patented (region 1, Figure 2a). A collimated beam is sufficient as an incident beam for the redesigned probe (region 1, Figure 2b). Applying a mirror-only system, chromatic aberration induced by the focusing lenses is also no longer present.

a
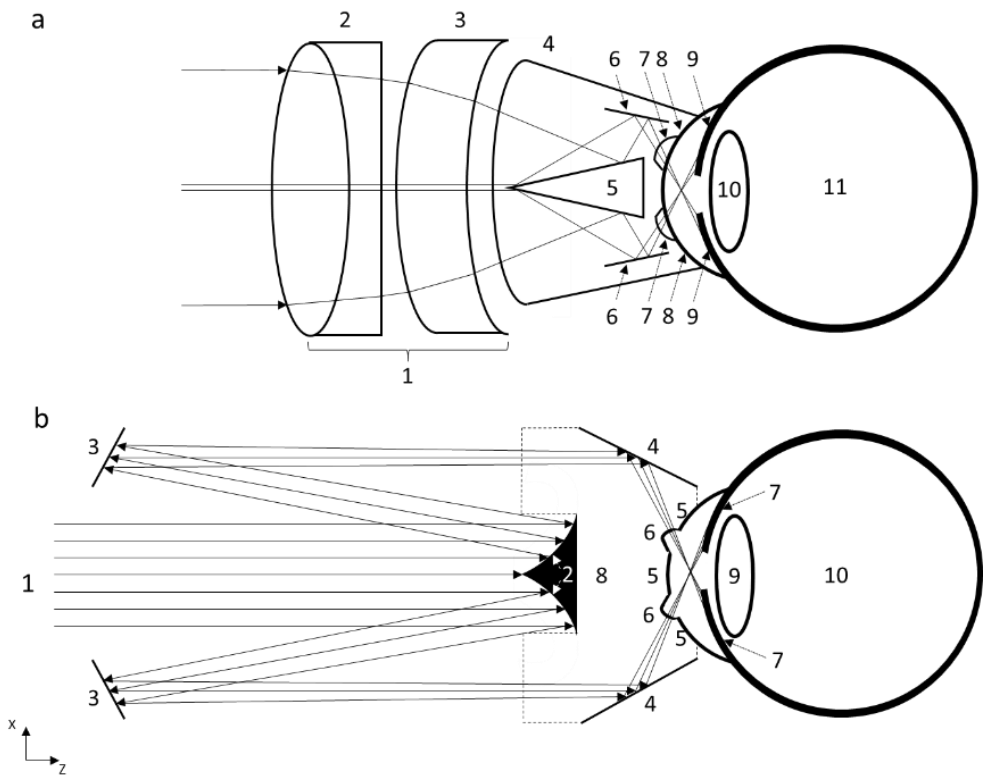

Fig. 2 Design of the ophthalmic probe. In former patented design(2a), a focusing lens (1) with an entry surface (2) and an exit surface (3) is used to provide focusing power and a spherical surface (4) adapted to the converging beam without adding further focusing power is also used to acting as a contact probe guides the light into the eye (11). An internal conical mirror (5) reflects the light to a further conical mirror (6) which further direct the light into the anterior eye chamber through an interface (7) and cornea surface (8). The excitation light will end on the iris (9), only scattering light reach the posterior part of the eye through the crystalline lens (10). 
In the newly designed probe (2b), the collimated incident light (1) directly reached an aspherical concave mirror (2) which provides focusing power of the probe. The reflected light is guided by a rotationally symmetric flat mirror (3) to feed into the main body of the probe (8) and direct to the eye (10) by surface (4) through an interface (6) on the corneal interface surface (5). The excitation light will end on the iris tissue (7), only scattering could reach the posterior part of the eye through the crystalline lens (9).

Figure $2 b$ shows a schematic representation of the newly designed probe. ${ }^{20}$ It is formed by three mirrors (2, 3 and 4), where surface 2 is an aspherical concave aluminum mirror to provide focusing power. The rotationally symmetric aspheric concave mirror can be described by:

$$
z=\frac{c r^{2}}{1+\sqrt{1-(1+k) c^{2} r^{2}}}+\beta_{1} r^{1}+\beta_{2} r^{2}+\cdots
$$

Here $c$ is the curvature (the reciprocal of the radius) and $r$ the radial coordinate. To have a focusing effect it implies that a parabola curve is needed for the two-dimensional cross-section curvature. Hence, the conic constant $k=-1$ and the equation can be written as:

$$
z=\beta_{1} r^{1}+\left(\frac{1}{2} c+\beta_{2}\right) \times r^{2}
$$

where $\beta_{1}$ and $\beta_{2}$ were both optimized and obtained by Zemax simulations. Surface 3 is a rotationally symmetric flat aluminum mirror that guide the laser beam to surface 4 properly. The mirror is tilted toward the curved mirror with 6.7 degrees and $7.5 \mathrm{~mm}$ long measured in cross section. The distance between surface 2 and surface 3 is also optimized by Zemax to ensure a focus in the central part of the AC. Surface 4 is an interface of poly(methyl methacrylate) (PMMA) and air, which functions as a mirror by total internal reflection.

The eye fitting part was carefully designed to fit the cornea. As shown in Figure 2b, the direction of the light beam guided by mirror 3 and surface 4 was designed such that it reaches the eye fitting surface perpendicularly. This to avoid extra refraction and minimize power loss. The diameter of the eye fitting component was $26.3 \mathrm{~mm}$ for the outer diameter of the probe and $15 \mathrm{~mm}$ for the eye fitting part respectively. Its length was $7.5 \mathrm{~mm}$ in total. Surface 4 was tilted $51^{\circ}$ from the horizontal surface to obtain total internal reflection in the PMMA body of the design. Through a special designed interfacial surface ( 6 in Figure $2 b$ ) with a radius of $8 \mathrm{~mm}$ and a chord length of $0.6 \mathrm{~mm}$ astigmatism caused by the corneal surface could be avoided. The laser will focus in the AC $1.2 \mathrm{~mm}$ deep under the top of corneal surface. Hereafter, the light will be absorbed in the region between cornea and iris. We assumed an $8 \mathrm{~mm}$ pupil, the edge of which also being the edge of the incident beam as shown in surface 7 in Figure 2b. Finally, the eye fitting surface was designed with a radius 
of $7.9 \mathrm{~mm}$ to match the curvature of the cornea. For patient comfort, all corners are rounded with varies of radius respectively.

\section{Prototype manufacture}

Based on the optimization by ray-tracing the optical design as explained above was used to produce a prototype of the probe (Figure 3). The central focusing mirror (surface 2 in Figure $2 b$ ) and the conical shaped mirror (surface 3 in Figure 2b) were made by polished aluminum to obtain a high reflectivity and quartz coating to prevent oxidation. The PMMA body of the probe with a refractive index of 1.487 at $671 \mathrm{~nm}$ and 1.485 at $785 \mathrm{~nm}^{21}$ ensures total internal reflection at surface 4 and transmission at interface 6 . All parts of the probe were produced using a high precision lathe.

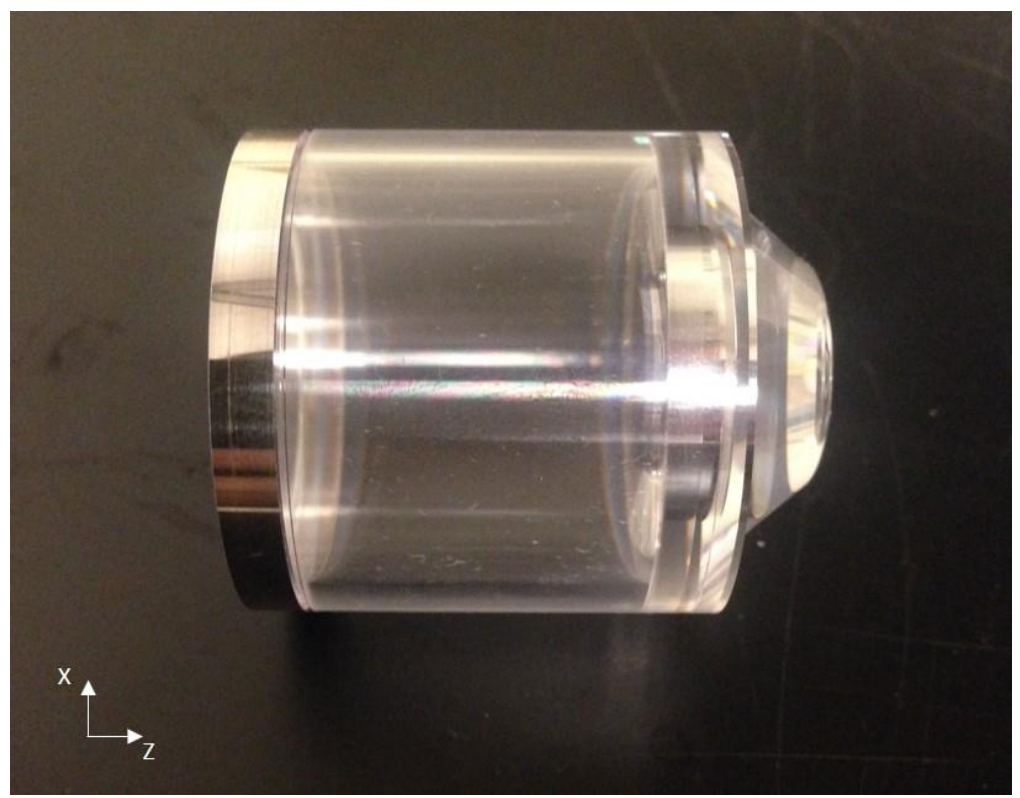

Fig. 3 Assembled prototype probe.

\section{Measurement}

\section{Confocal Raman spectroscopy}

To bring Raman spectroscopy into the clinic, a compact and mobile spectrometer was used. Figure 4 shows the schematic representation of the confocal Raman spectroscopy (CRS) setup. It consists of a Raman spectrometer with two integrated excitation sources, one for the fingerprint region and one for the longer wavelength shift, a dark-field eye probe and a sample stage. The confocal setup with $25 \mu \mathrm{m}$ pinhole minimizes possible contributions of out of focus signals that are generated in tissues along the path way. ${ }^{22}$ 

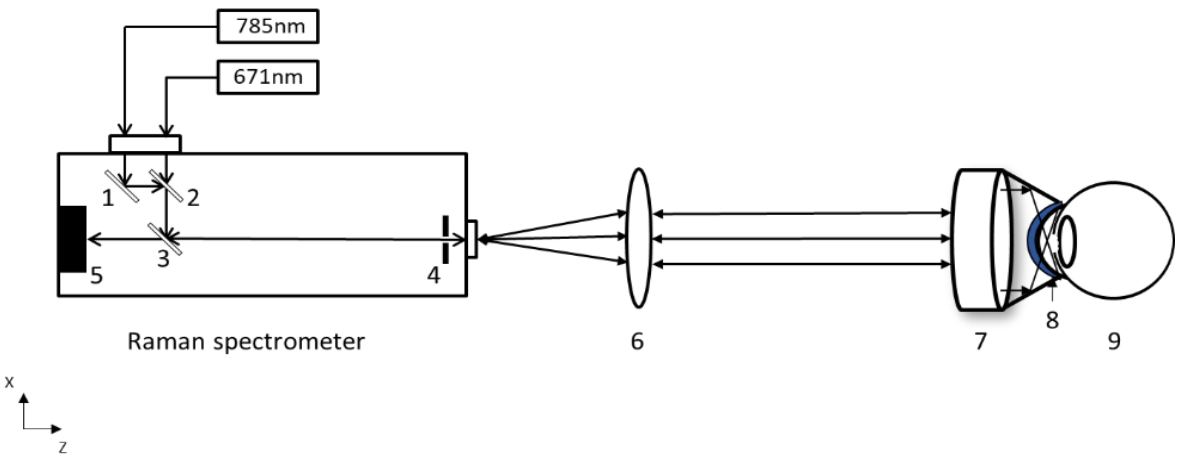

Fig. 4 Schematic of the optical setup. In Figure 4 the circle symmetric probe, the diode lasers $(671 \mathrm{~nm}$ and $785 \mathrm{~nm}$ ) are coupled on one optical axis by a mirror (1) and dichroic mirror (2) and (3) within the spectrometer. The excitation light leaves the spectrometer through an integrated $25 \mu \mathrm{m}$ pinhole (4). An $\mathrm{f}=80 \mathrm{~mm}$ achromat (6) collimates the excitation beam before it enters the on a gimbal mounted probe (7). The eye (9) is optically coupled to the probe with an ophthalmic gel (8). The back scattered Raman radiation that fits into the NA=0.05 optical system is transported back into the spectrometer and recorded by a CCD (5). Due to this coincidence set up, the spectrometer facilitates a confocal isolation of the aqueous humor from the about $100 \times$ stronger Raman signal from the corneal tissue.

As excitation sources a $15 \mathrm{~mW}$ diode laser with a wavelength of $785 \mathrm{~nm}$ (Innovative Photonic Solutions SM $785 \mathrm{~nm}$, Monmouth Junction, United States) and a $20 \mathrm{~mW}$ diode laser with a wavelength of $671 \mathrm{~nm}$ (Laser Quantum Ignis 671 and SMD 6000, Konstanz, Germany) were utilized. A High-Performance Raman spectrometer (Module Model 2500, River Diagnostics ${ }^{\circledR}$, Rotterdam, The Netherlands) was employed with a charge-coupled device camera to record the Raman spectra at a working temperature of $-65^{\circ} \mathrm{C}$. The module receives the laser excitation light through two single-mode diamond optical fibers. A $25 \mu \mathrm{m}$ pinhole was placed in the spectrometer to allow the laser beams - that have a waist of about $18 \mu \mathrm{m}$ - pass into the measurement area. An $80 \mathrm{~mm}$ focal length doublet lens was placed after the spectrometer aperture to change the diverging beam $(\mathrm{NA}=0.05)$ into an $8 \mathrm{~mm}$ diameter parallel beam. Alignment of the $80 \mathrm{~mm}$ collimating lens was achieved with a shearing interferometer to obtain a perfect parallel excitation beam that is coupled into the eye probe.

The eye probe was mounted in a Gimbal mirror mounts (BHAN-50M, OptoSigma ${ }^{\circledR}$, Les Ulis, France), with fine adjustment in horizontal and vertical directions. Maximum adjustment range is $\pm 4^{\circ}$, resolution of rotation is $0.31^{\circ}$ and $0.48^{\circ}$ in $\mathrm{x}$ and $\mathrm{y}$ axis separately. Alignment in the sample was performed by translation and rotation adjustments of the gimbal until the maximum signal appeared.

All Raman spectra were processed by a self-developed MATLAB program (Version 2017b, The Mathworks Inc., Natick, USA). ${ }^{23}$ 


\section{Sample preparation}

Enucleated eyes from sacrificed New-Zealand white rabbits were stored in $-80{ }^{\circ} \mathrm{C}$ freezers before use. The eyes were thawed at room temperature then submerged in phosphate buffered saline (PBS, $\mathrm{pH}$ 7.4) to prepare for use. $150 \mu$ l volume AH was removed from the rabbit eye by a syringe with $30 \mathrm{G}$ needle. Subsequentially the same volume of Phenylephrine $\mathrm{HCl} \mathrm{10 \%} \mathrm{(Bausch} \mathrm{\&} \mathrm{Lomb} \mathrm{U.K} \mathrm{Limited,} \mathrm{Surrey,} \mathrm{UK)} \mathrm{was}$ injected by another $30 \mathrm{G}$ needle syringe into the AC. All animal sample procedures were conducted under the Guidelines of the Central Laboratory Animal Facility of Maastricht University and complied with the ARVO Statement for the Use of Animals in Ophthalmic and Visual Research. All protocols were approved by the Central Committee for Animal research and were in accordance with the European Guidelines (2010/63/EU).

For the prototype probe as shown in Figure 3, topically applied Methocel $^{\circledR} 2 \%$ (OmniVision, Santa Clara, CA, USA) was used as an ophthalmic gel. It functions both to moister the cornea and to remove air between the probe and the eye. The gel has a refractive index of 1.469 , which also bridges the refraction index difference of the probe (1.487) and cornea (1.376).

\section{Results and discussions}

\section{Ray-trace simulation}

In Figure 5a, the spot diagram shows that the Root-Mean-Square (RMS) radius of the excitation light $(785 \mathrm{~nm}$ ) focus in the $\mathrm{AC}$ is $6.684 \mu \mathrm{m}$ and the Geometric (GEO) radius is $18.577 \mu \mathrm{m}$. The RMS radius gives a rough idea of the spread of the focus spot while the GEO radius indicates the distance of farthest illuminated boundary from the center. Due to the small aperture and the long wavelength this is an about diffraction limited spot. Figure $5 \mathrm{~b}$ shows the cumulative normalized energy as function of the encircled radius. When properly aligned nearly all energy was collected within a $12 \mu \mathrm{m}$ radius (triangle labeled line). In case of a severe $(3.5 \mathrm{~mm})$ lateral misalignment with the probe the ray-traced focus showed a scattered power distribution in a broad area outside the focal area (dots labeled line), However, with already a slight rotation misalignment of $0.3^{\circ}$ in one direction, no energy at all is collected within a $12 \mu \mathrm{m}$ radius (square labeled line). A recognizable signal can only be obtained with a $100 \mu \mathrm{m}$ radius, implying that the widths of the pinhole of the spectrometer needs to be $200 \mu \mathrm{m}$ to collect a considerable amount of the Raman back scattered light. However, a $200 \mu \mathrm{m}$ pinhole destroys the confocal effect necessary to limit the integration length in the $\mathrm{AH}$.

The simulation indicates the extreme sensitivity for rotation misalignment. Under such a misalignment the focus of the excitation light is displaced and disrupted into scattered elements, hence the collected Raman signal obtained from such an element will be strongly decreased and will not be a guidance to proper focusing. The decreased maximum intensity 
after the focus and the disrupted intensity distribution will minimize the light damage of the tissue accordingly.
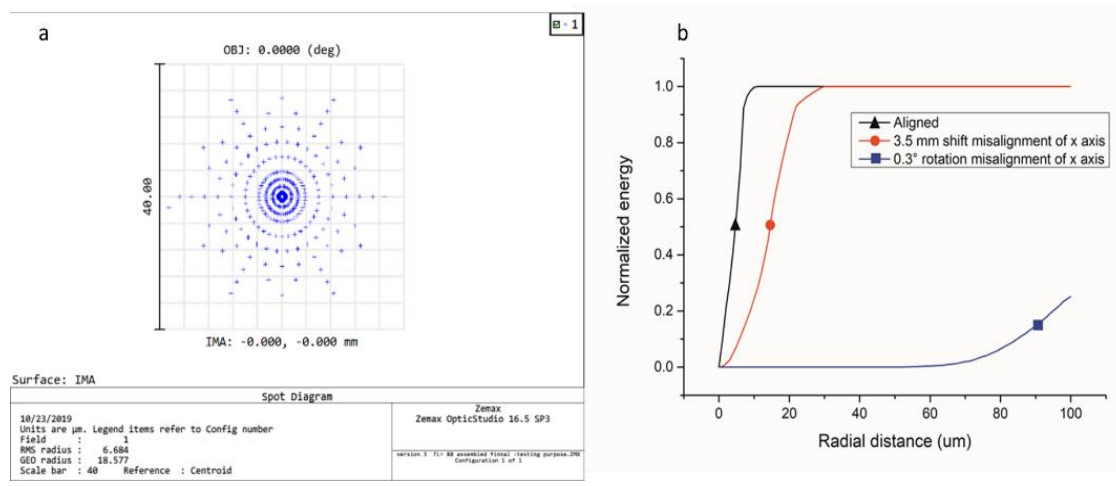

Fig. 5 Simulation result of the newly designed probe. In figure 5a, the spot diagram shows that the RMS radius of the focus is $6.684 \mu \mathrm{m}$ and the GEO radius is $18.577 \mu \mathrm{m}$. In figure $5 \mathrm{~b}$, the encircled radius of cumulative normalized energy diagram shows that aligned probe (triangle labeled line) could collect nearly all energy within a $12 \mu \mathrm{m}$ radius; $3.5 \mathrm{~mm}$ lateral misalignment (dots labeled line) could collect nearly all energy within a $30 \mu \mathrm{m}$ radius; a rotation misalignment of $0.3^{\circ}$ lead to a recognizable signal with around $100 \mu \mathrm{m}$ radius.

\section{Raman spectrum}

The design of prototyped probe for measuring the Raman spectrum of the aqueous humor in a "dark field" modality was tested on ex vivo rabbit eyes. To avoid the 100 times stronger signals of the surrounding tissues, a confocal setup was applied by using a spectrometer of which the excitation light as well as the Raman signal were passing the same $25 \mu \mathrm{m}$ pinhole. In Figure 6a, a peak at the wavenumber of $1002 \mathrm{~cm}^{-1}$ is observed with an exposure time of 90 seconds from the $\mathrm{AH}$ of a rabbit eye with Phenylephrine injection. The peak position is the same as the ocular drug Phenylephrine which formerly reported by Elshout, M. et al.. ${ }^{24}$ In Figure 6b, the AH showed a spectral response from the $\mathrm{OH}$ bending mode of water at an exposure time of 60 seconds.
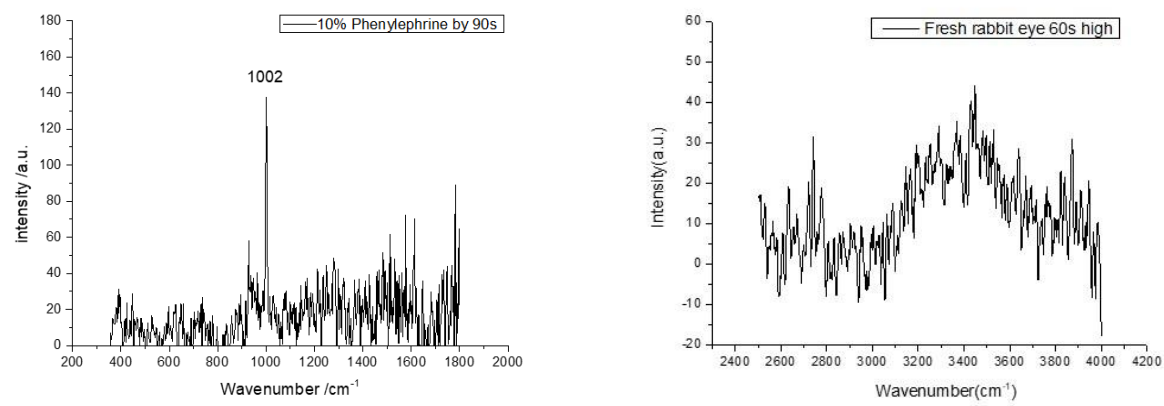
Fig.6 Raman spectrum obtained by the prototype probe with a) $10 \%$ phenylephrine in 90 s integration time and b) water $\mathrm{OH}$ bending mode in 60 s integration time.

Although the prototyped probe showed a recognizable Raman signal, it was by far not the signal that could be easily obtained due to the alignment difficulties which is also revealed by the simulation results. More effort was needed to achieve this alignment, which is an unsurmountable obstacle for ophthalmologists during clinical practice.

\section{Data processing}

Aqueous humor contains variant compounds including inorganic ions and organic anion, carbohydrates, glutathione and urea, proteins, growth-modulatory factors, oxygen, and carbon dioxide. ${ }^{25}$ Not all of them are Raman active since the Raman scattering mainly related to the vibrational, rotational and other low-frequency modes of intramolecular bonds. These compounds in $\mathrm{AH}$ are dynamically interchanging with many tissues inside the eye, but $\mathrm{AH}$ is in a closure environment with stable exchange rate of $2-3 \mu 1 / \mathrm{min}$ in a health human eyes ${ }^{1}$ and exchange rate of 2.6-3.6 $\mu \mathrm{l} / \mathrm{min}$ in rabbit eyes ${ }^{26}$ which can be considered as static comparing with the Raman measurement time. In a multiple chemical mixture environment like $\mathrm{AH}$, substance identification only by single peak might lead to a biased conclusion because some composition might share some chemical bonds in common. However, there is not a well-accepted data processing procedure available yet. ${ }^{27}$ In a previous study, we proposed and validated a MATLAB program for data processing of Raman detection of ocular drug topically delivered to the animal eyes in vitro and in vivo. ${ }^{23}$ It demonstrated that with proper data processing techniques, Raman spectroscopy could be used in ocular tissue investigation with an ophthalmic probe.

\section{Conclusion}

In this study, simulation analysis and in vitro tests indicated that the dark-field probe as prototyped is suitable for applying on human eyes. Recognizable Raman signals were acquired from the ex vivo rabbit eyes as proof of principle. This design demonstrated the potential for real-time, noninvasive Raman aqueous humor measurements. However, difficulties to achieve alignment hinders its clinical application and further improvement is needed. Development of a probe with a less critical rotational alignment and of which the signal during the alignment guides the operator to a proper alignment will be the next step in our research.

\section{Acknowledgments}

We would like to thank Sumipro B.V. for their advice while manufacturing the prototype and Theresa Kocanda for her help while evaluating the performance of probe prototype. We also would like to thank Christian J.F. Bertens for his help while obtaining the OCT image of the rabbit eye in Figure 1. Shuo Zhang acknowledges the China Scholarship Council for the support by State Scholarship Fund No. 201309110103. 


\section{Disclosures}

The authors declare that there are no conflicts of interest to disclose.

Page | 102 


\section{References}

1. L. M. Levine, "Basic and Clinical Science Course, Section 2: Fundamentals and Principles of Ophthalmology," in Basic and Clinical Science Course, p. 430, American Academy of Ophthalmology (2018-2019).

2. S. H. Yun, and S. J. J. Kwok, "Light in diagnosis, therapy and surgery," Nature Biomedical Engineering 1(1), 0008 (2017).

3. R. J. Erckens et al., "Raman spectroscopy in ophthalmology: from experimental tool to applications in vivo," Lasers Med. Sci. 16(4), 236-252 (2001).

4. S. Pohjola, "The glucose content of the aqueous humour in man," Acta Ophthalmol (Copenh) Suppl 88:81-80 (1966).

5. M. Reim et al., "Steady state levels of glucose in the different layers of the cornea, aqueous humor, blood and tears in vivo," Ophthalmologica 154(1), 39-50 (1967).

6. C. C. Pelletier, J. L. Lambert, and M. Borchert, "Determination of glucose in human aqueous humor using Raman spectroscopy and designed-solution calibration," Appl. Spectrosc. 59(8), 1024-1031 (2005).

7. T. Kokubun et al., "Characteristic profiles of inflammatory cytokines in the aqueous humor of glaucomatous eyes," Ocul Immunol Inflamm 26(8), 1177-1188 (2018).

8. J. J. Kuiper et al., "An ocular protein triad can classify four complex retinal diseases," Sci Rep 7(41595 (2017).

9. K.-H. D. Michael Kaschke, Michael Stefan Rill, Optical Devices in Ophthalmology and Optometry: Technology, Design Principles and Clinical Applications, Wiley (2014).

10. A. R. Boyd, G. A. Burke, and B. J. Meenan, "Monitoring cellular behaviour using Raman spectroscopy for tissue engineering and regenerative medicine applications," J Mater Sci Mater Med 21(8), 23172324 (2010).

11. P. Chen et al., "Bio-Raman spectroscopy: a potential clinical analytical method assisting in disease diagnosis," Analytical Methods 3(6), 1257 (2011).

12. I. Pence, and A. Mahadevan-Jansen, "Clinical instrumentation and applications of Raman spectroscopy," Chem Soc Rev 45(7), 1958-1979 (2016).

13. K. J. I. Ember et al., "Raman spectroscopy and regenerative medicine: a review," npj Regenerative Medicine 2(1), (2017).

14. Z. Movasaghi, S. Rehman, and I. U. Rehman, "Raman spectroscopy of biological tissues," Applied Spectroscopy Reviews 42(5), 493-541 (2007).

15. F. H. Jongsma et al., "Confocal Raman spectroscopy system for noncontact scanning of ocular tissues: an in vitro study," Optical Engineering 36(11), 3193-3200 (1997).

16. J. J. Vos, and D. van Norren, "Retinal damage by optical radiation. An alternative to current, ACGIHinspired guidelines," Clin. Exp. Optom. 88(4), 200-211 (2005).

17. P. International Commission on Non-Ionizing Radiation, "ICNIRP Guidelines on Limits of Exposure to Laser Radiation of Wavelengths between $180 \mathrm{~nm}$ and 1,000 mum," Health. Phys. 105(3), 271-295 (2013).

18. H. L. Liou, and N. A. Brennan, "Anatomically accurate, finite model eye for optical modeling,".J Opt. Soc. Am. A. Opt. Image. Sci. Vis. 14(8), 1684-1695 (1997).

19. J. De Brabander, and F. H. M. Jongsma, "Device and method for performing measurements of the chemical composition of the anterior eye," E. P. Office, Ed. (2008).

20. T. T. T. J. M. B. Shuo Zhang, Roel Johan Erckens, Franciscus Hermanus Maria Jongsma, John De Brabander "Device for performing measurements of the chemical composition of the anterior eye as well as an integrated optical unit for implementation therein," E. p. office, Ed., p. 29 (2018).

21. M. Szczurowski, "Refractive index and related constants - Poly(methyl methacrylate) (PMMA, Acrylic glass)," pp. Refractive index and related constants - Poly(methyl methacrylate) (PMMA, Acrylic glass). Refractiveindex.info. (2013).

22. M. Minsky, "Memoir on inventing the confocal scanning microscope," Scanning 10(4), 128-138 (1988).

23. C. J. F. Bertens et al., "Confocal Raman spectroscopy: Evaluation of a non-invasive technique for the detection of topically applied ketorolac tromethamine in vitro and in vivo," Int. J. Pharm. 570(118641 (2019).

24. M. Elshout et al., "Detection of Raman spectra in ocular drugs for potential in vivo application of Raman spectroscopy," J. Ocul. Pharmacol. Ther. 27(5), 445-451 (2011).

25. K. V. Chalam, "Basic and Clinical Science Course, Section 2: Fundamentals and Principles of 
Ophthalmology," in Basic and Clinical Science Course, p. 455, American Academy of Ophthalmology (2013-2014).

26. S. D. Smith, "Measurement of the rate of aqueous humor flow," Yale J Biol Med 64(1), 89-102 (1991).

27. H. J. Byrne et al., "Spectral pre and post processing for infrared and Raman spectroscopy of biological tissues and cells," Chem Soc Rev 45(7), 1865-1878 (2016). 


\section{Chapter 6}

Design and performance of a dark field probe with confocal Raman spectroscopy for ophthalmic applications

Shuo Zhang, Roel J. Erckens, Frans H.M. Jongsma, Carroll A.B. Webers and Tos T.J.M. Berendschot Journal of Raman Spectroscopy 2021;1:5 DOI: $10.1002 / j r s .6125$ 


\begin{abstract}
A darkfield ophthalmic contact probe for analysis of aqueous humor (AH) was designed by ray-tracing and constructed in quartz glass for experimental use as a prototype probe in a confocal Raman spectroscopic setup. The misalignment tolerance of this probe was theoretically evaluated while the optical performance was experimentally evaluated by a concentration curve of the ocular drug phenylephrine $\mathrm{HCl}$ in an artificial eye model. The results indicated a 9 fold improvement in misalignment tolerance and a 36 fold improvement in the signal-to-noise ratio at the phenylephrine $\mathrm{HCl} 1002 \mathrm{~cm}^{-1}$ peak compared to a previous design. The prototype showed a good linear fit with the phenylephrine $\mathrm{HCl}$ concentration, with an $\mathrm{R}$ square of 0.996. Phenylephrine $\mathrm{HCl}$ concentration down to $0.1 \%$ is detectable.
\end{abstract}

\title{
Key words
}

Raman spectroscopy, Aqueous Humor, Ocular Drug, Dark-Field Illumination 


\section{Introduction}

Raman spectroscopy (RS) applications in tears, aqueous humor (AH), and blood have been rapidly increasing in recent years. ${ }^{1-4}$ Real-time investigations by $\mathrm{RS}$ in these biofluids reveal crucial information about physiological and pathological changes and diseases development. RS has non-invasive and non-destructive capabilities that are preferred in real-time examinations, especially in ophthalmology clinical practices where an invasive biopsy is currently the standard method to withdraw AH. Invasive procedures have several drawbacks, not only are they a burden for patients, but they also increase the risk of inflammation.

The anterior segment of the eye mainly consists of the cornea, AH, iris, ciliary body (CB) and the lens. The ocular fluid in the AH is produced by the ciliary body (CB) and provides nutrition to the cornea and lens. The $\mathrm{AH}$ is mainly water, along with inorganic ions, organic anions, glucose, carbohydrates, glutathione and urea, proteins, growthmodulatory factors, oxygen, and carbon dioxide. AH has a slowly varying dynamic equilibrium, which might interference with the molecule concentration measurement. However, the dynamic change rate is $2 \mu \mathrm{l} / \mathrm{min}$ with a total volume of $\mathrm{AH}$ is approximately $250 \mu \mathrm{L}$, which can be neglected comparing to our measurement time. The AH's composition ratios and concentrations reveal part of the physiological status of the internal ocular system. Imbalance in concentrations can be indicative of systematic disease or dysfunction of the visual system. ${ }^{5}$ For this reason, RS has been used to investigate glucose levels in $\mathrm{AH}$ as it may be an indicator of diabetes complications. ${ }^{6}$

Excitation with laser light is an indispensable technique in acquiring a high signal-tonoise ratio (SNR) in Raman spectroscopy. However, direct laser radiation that reaches the retinal tissue might cause irreversible light damage when employing laser light along the eye optical axis. ${ }^{7,8}$ Therefore, legal guidelines limit the possibility to improve Raman signal by increasing the laser power in a direct illumination approach for ophthalmological applications. To prevent a light hazard to the retina tissues and guarantee a safe usage of $\mathrm{RS}$, the principle of a 'dark field' illumination technique used in microscopy was proposed. In a conventional 'dark field' optical setting a center blocked aperture placed in the excitation beam and a high Numerical aperture (NA) objective lens is needed. ${ }^{9}$ The center blocked aperture prevents the direct illumination along the optical axis from entering the pupil, however, the loss of a large portion of the excitation light in the center results in a significant decrease of the Raman signal's intensity. In our previous work, a functioning 'dark field' Raman eye probe with improved excitation light utilizing efficiency was applied..$^{10}$ However, since that probe's Raman signal intensities were below expectation and almost impossible to align, we set out to redesign the probe.

In this paper, we describe the redesigned 'dark field' illumination eye probe and demonstrate its performance. This design increases the alignment tolerance and improves the performance of the previous probe significantly which could better achieve the goal of preventing the excitation laser light from directly reaching retinal tissue. 


\section{Methods}

Fig. 1a shows a schematic representation of the confocal Raman spectroscopy (CRS) setup combined with the confocal probe. ${ }^{10,11}$ A $26 \mathrm{~mW}$ diode $785 \mathrm{~nm}$ laser was utilized as a Raman excitation light source (Innovative Photonic Solutions SM $785 \mathrm{~nm}$, Monmouth Junction, NJ, US). A commercial Raman spectrometer (model 2500, River Diagnostics ${ }^{\circledR}$, Rotterdam, the Netherlands) was used to acquire the Raman spectra. The spectrometer received the laser through a single-mode optical fiber and guided the beam output through an integrated $25 \mu \mathrm{m}$ pinhole with a NA of 0.05 . The pinhole in the confocal setup was used for suppressing the out of focus signals generated in tissues along the pathway. A chargecoupled device camera (CCD) was integrated within the spectrometer for Raman signal detection. To improve the sensitivity, the $\mathrm{CCD}$ was air-cooled to $-60{ }^{\circ} \mathrm{C}$ for decreasing its thermal noise. A diverging laser beam out of the spectrometer is converted to a collimated beam by an $80 \mathrm{~mm}$ focus length lens (f80) with diameter of $20 \mathrm{~mm}$. A $60 \mathrm{~mm}$ focal length lens (f60) with diameter of $20 \mathrm{~mm}$ provided focusing power in front of the probe. The CRS system was used in the single-point modus. Raman spectra were observed in the wavenumber range of $350 \mathrm{~cm}^{-1}$ to $1800 \mathrm{~cm}^{-1}$.

Phenylephrine HCL is a commonly used ocular drug for pupil dilation in eye examinations and has recognizable Raman peak at $1002 \mathrm{~cm}^{-1}{ }^{12}$ The phenylephrine $\mathrm{HCl}$ (Laboratoires Théa; Monofree Phenylephrine $\mathrm{HCl} 5 \%$, Clermont-Ferrand, France) were diluted from clinically applied concentration 5\% into the following concentrations: $1 \%$, $0.75 \%, 0.5 \%, 0.25 \%, 0.1 \%$, and $0.05 \%$. All samples were measured in a custom made artificial eye model (AEM) to attain a concentration curve and determine the detection limit with the 3-sigma criterion. ${ }^{13}$ The custom made AEM consists of a transparent surface with the same curvature as the cornea $(7.9 \mathrm{~mm}$ radius) and a sealed container which can be filled with various chemical solutions. All spectra were obtained with 60 seconds integration time averaged 3 times. A water spectrum was used for background subtraction.

\section{Design and manufacture of the probe}

In our previous design (Fig. 1b), the collimated incident light (A, Fig.1b) directly reached an aspherical concave mirror (B, Fig.1b) which provides the focusing power of the probe. The reflected light is guided by a rotationally symmetric flat mirror (C, Fig.1b) to feed into the main body of the probe (D, Fig.1b) and direct to the eye (J, Fig.1b) by a total internal reflection mirror (E, Fig.1b) through an incident window (G, Fig.1b) and enter an interface (F, Fig.1b) between the probe and cornea. The excitation light will end on the iris (H, Fig.1b), only scattering could reach the posterior section of the eye (J, Fig.1b) through the crystalline lens (I, Fig.1b). The dark field illumination principle was achieved by having the incident laser light passing through the periphery of the cornea and focusing on the AH. As shown in Fig. 1b, the excitation laser light goes through the $\mathrm{AH}$ and ends on the iris $(\mathrm{H}$, Fig.1b), only scattered light can reach the posterior section of the eye through the crystalline lens (I, Fig.1b). However, the previous design was very difficult to align and 
mainly due to this problem the SNR of the obtained Raman signal was unsatisfying. Other drawbacks might be due to the following reasons. First, the mirror deviation angle caused by misalignment is amplified two times on each mirror in a multilevel reflective optical system. Furthermore, the misalignment is destroying the focusing function provide by the aspherical concave mirror (B, Fig.1b). In addition, reflection loss occurs at Aluminum (Al) coated mirrors (B, C, Fig.1b). The reflectance of Al mirrors at $785 \mathrm{~nm}$ is 0.873 , there are two reflectance (each time at $\mathrm{C}, \mathrm{E}$, Fig.1b) for the excitation light path and two reflectance (each time at C, B, Fig.1b) when collecting signals in the same path (detection path) in a confocal setup. Hence, we expected a Raman signal loss due to the other mentioned drawbacks of about $50 \%$. However, we obtained less than $1 \%$ of a signal that we obtained with a single lens and cuvette filled with a target fluid.

In our redesigned prismatic probe, the issue of misalignment and low SNR are properly addressed by removing two Al mirrors. The tolerance of the alignment improved considerably by reducing the number of mirrors. The angle that can acquire the Raman signal hence increased at least four times without the two times applications occurred on each Al mirror. In addition, the power loss that occurred on these Al mirrors is eliminated by the removal, which therefore improved the SNR as the Raman signal intensity is proportional with the laser power. The total internal reflection mirror (E', Fig.1c) has been maintained in the current design with an adapted angle. There are two benefits. First, no power loss occurs on this surface by the principle of total internal reflection. Second, the excitation beam output can be used for the probe position check. The output laser reflected by the opposite side of the surface can be observed at a screen placed at position L in Fig. 1c. The quartz-air interface (E', Fig. 1c) is tilted 48.26 degrees from the horizontal surface to obtain total internal reflection. For $785 \mathrm{~nm}$, the critical angle is 43.47 degrees. The angle was selected as a result of simulation optimization, assuming perpendicular incident excitation light of the probe front surface (B, Fig. 1c). A Liou-Brennan eye model was used in our dark-field design during the whole simulation procedure. ${ }^{14}$ All ray-tracing simulations were done at $785 \mathrm{~nm}$. The eye fitting part (F, Fig. 1c) was designed with a radius of $7.9 \mathrm{~mm}$ to fit the curvature of the cornea. The geometrical integration length of the excitation light based on the diameter of the pinhole and the NA of the spectrometer is about 1 millimeters with a $25 \mu \mathrm{m}$ pinhole and is nearly perpendicular to the optical axis of the eye. 

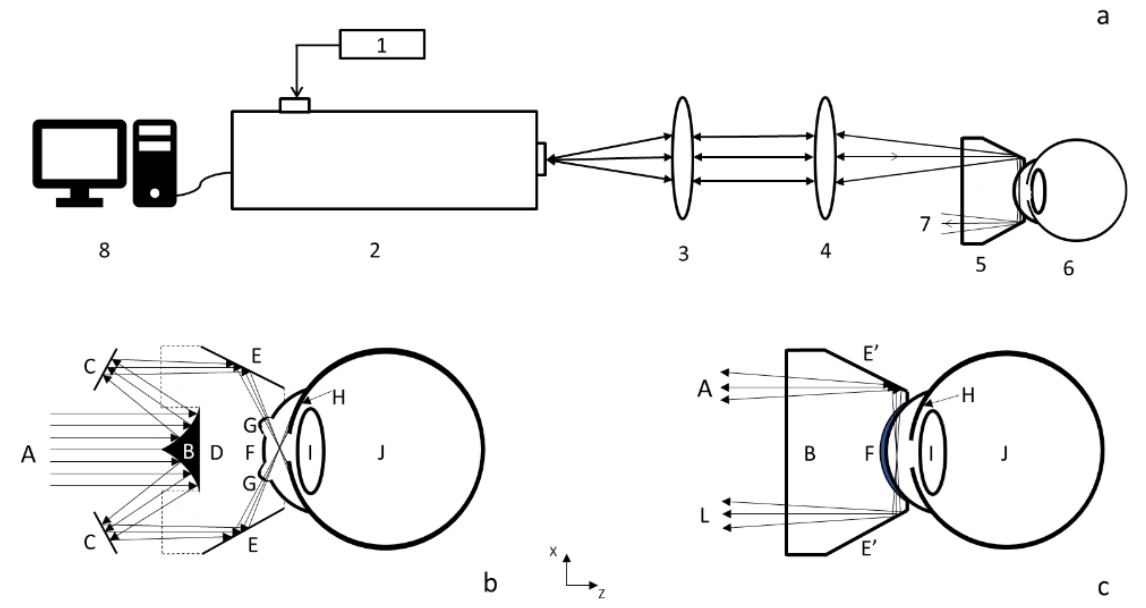

Figure 1. Raman system and the design of the ophthalmic probe. a) Confocal Raman spectroscopy system. (1) The diode lasers $785 \mathrm{~nm}$. (2) Raman spectrometer. (3) An f=80mm achromat. (4) A f=60mm achromat. (5) The ophthalmic probe. (6) The eye. (7) The excitation beam output. (10) Computer. b) Previously reported design. (A) The collimated incident light. (B) An aspherical concave mirror. (C) A rotationally symmetric flat mirror. (D) The main body of the probe that manufactured by PMMA. (E) Total internal reflection mirror (interface between the probe and the air). (F) An interface between the probe and corneal. (G) Incident window. (H) Iris. (I) the crystalline lens. (J) The posterior section of the eye. c) Newly designed probe. (A) The collimated incident light. (B) The main body of the probe that manufactured by quartz. (E') Total internal reflection mirror (interface between the probe and the air). (F) An interface between the probe and corneal surface. (H) Iris. (I) the crystalline lens. (J) The posterior section of the eye. (L) The excitation beam output.

A prototype of the probe was manufactured based on the focus performance optimization via ray-tracing software Zemax (version 16.5 Sp3, Zemax LLC, Kirkland, Washington) and the optical design described above. The probe body was made of quartz with a refractive index of 1.454 at $785 \mathrm{~nm}$. This ensured total internal reflection at surface E' in Fig. 1c and transmission at the front surface of the probe. Alignment tolerance was evaluated by Ray tracing software and Raman signal intensities were determined using an ocular drug in the AEM.

\section{Results and discussion}

\section{Ray-trace Simulation}

Alignment tolerance with different angles of the incident light shows a 0.75 degree angle towards the cornea and a 1.05 degree angle towards the lens relative to the designed focal point in the anterior chamber of the eye. A Gonio lens for ophthalmic examination is also able to prevent the laser from direct illumination on the retina when used in conjunction with Raman spectroscopy. ${ }^{4}$ Therefore, alignment tolerance simulations of a Gonio lens (Haag-Streit Meridian; CGA1, Köniz, Switzerland) were also calculated. The results showed that the alignment within \pm 1.5 degrees is within the safety range of the 
dark illumination indicating that the proposed probe's alignment requirement is comparable with commercial products used in clinical practice. Compared with our previous probe, the simulation results indicate that our current design has 9 fold improvement in alignment tolerance. Furthermore, unlike the Gonio lens where the laser ends on the iris, the proposed design has a beam output to the free space (L, Fig. 1c) for an alignment check on a screen or plane.

\section{Background subtraction}

Raman scattering of quartz has a strong band centered around $442 \mathrm{~cm}^{-1}$. However, the phenylephrine HCL target Raman band at $1002 \mathrm{~cm}^{-1}$ does not interfere with either the water or quartz spectra. For other spectral regions, the confocal setup significantly suppresses the Raman signal generated by quartz in the excitation pathway. We used the water spectrum for subtraction, since the ocular drug is diluted by the water and no other major peaks were identifiable from additives. Therefore, when subtracting the water spectrum, interference including fluorescence and possible minor peaks from the additives are removed. In addition, the water and phenylephrine HCL spectra both were the average of three frames before further data processing procedures were applied. Hence, additional noise that might be introduced is minimized.

\section{Ocular drug measurement in AEM}

Raman spectra obtained in the artificial eye of phenylephrine $\mathrm{HCl}$ with various concentrations were obtained in conjunction with the CRS system. The major peak at 1002 $\mathrm{cm}^{-1}$ could easily be identified (Fig. 2b). Compared to the previous probe, the SNR of this peak improved 36 fold. Phenylephrine $\mathrm{HCl}$ with concentrations of $1 \%, 0.75 \%, 0.5 \%$, $0.25 \%, 0.1 \%$, and $0.05 \%$ were measured (Fig. 2c). A linear fit showed an R-square of 0.996 .

In clinical practice, phenylephrine $\mathrm{HCl}$ is topically administered. Typically, less than $5 \%$ of the applied dose of the ocular drug will cross the ocular barriers and reach the anterior chamber. ${ }^{15}$ Hence, the detection limit of Raman systems needs to be lower than $0.25 \%$, as a concentration of $5 \%$ phenylephrine $\mathrm{HCl}$ is normally provided in clinic practice. Furthermore, compared to the AEM, in vivo measurements might encounter more interference or signal loss due to inhomogeneous optical properties in corneal tissue. We lowered the concentration measurements down to $0.05 \%$ and managed to obtain reliable measurement (SNR above 3) from the concentration of $0.1 \%$, which could leave some sensitivity availability for future applications in vitro and in vivo. 

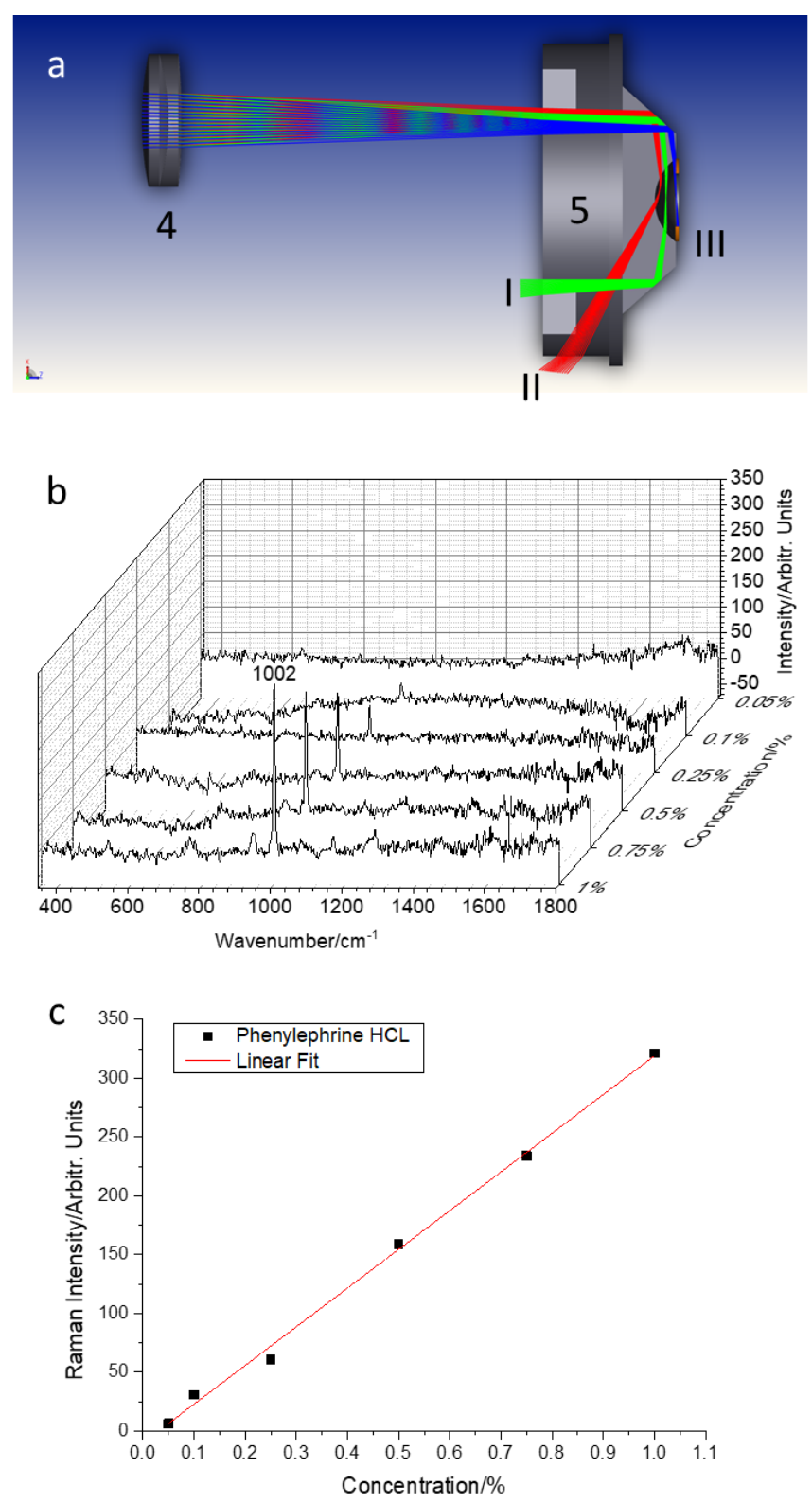

Figure 2. The simulation results of the misalignment and the Raman spectrum of phenylephrine $\mathrm{HCl}$ with the linear fit of the phenylephrine $\mathrm{HCl}$ characteristic peak at $1002 \mathrm{~cm}^{-1}$. a) The simulation results of misalignment. The excitation beam through a $\mathrm{f}=60 \mathrm{~mm}$ achromat lens (4) and enter the probe (5) under the condition of an aligned (I), a 0.75 degrees towards the cornea (II) and a 1.05 degrees towards the lens (III). b) Raman spectrum of phenylephrine $\mathrm{HCl}$ with concentrations of $0.05 \%, 0.1 \%, 0.25 \%, 0.5 \%, 0.75 \%$, and $1 \%$. c) The linear fit of characteristic of phenylephrine $\mathrm{HCl}$ with concentrations of $0.05 \%, 0.1 \%, 0.25 \%, 0.5 \%, 0.75 \%$, and $1 \%$. 


\section{Conclusion}

A simplified ophthalmic probe with CRS was designed and validated for pre-clinical use. By using a lens and a prism assembly probe the misalignment tolerance improved 9 fold and the SNR at the ocular drug phenylephrine $\mathrm{HCl}$ characteristics peak improved 36 fold compared to a previous design. With this confocal darkfield probe, eye safe optical probing without obscured signals from adjacent tissues and the use of higher power laser light to increase sensitivity was shown to be possible.

\section{Funding}

The first author was supported by the China Scholarship Council under State Scholarship Fund No.201309110103.

\section{Disclosures}

The authors declare that there are no conflicts of interest to disclose.

\section{Acknowledgements}

The authors thank Jackson T. Boonstra for English editing. 


\section{Reference:}

1. J. Filik, and N. Stone, "Analysis of human tear fluid by Raman spectroscopy," Anal. Chim. Acta. 616(2), 177-184 (2008).

2. M. J. Baker et al., "Developing and understanding biofluid vibrational spectroscopy: a critical review," Chem. Soc. Rev. 45(7), 1803-1818 (2016).

3. J. L. Lambert, C. C. Pelletier, and M. Borchert, "Glucose determination in human aqueous humor with Raman spectroscopy," J. Biomed. Opt. 10(3), 031110 (2005).

4. C. J. F. Bertens et al., "Confocal Raman spectroscopy: Evaluation of a non-invasive technique for the detection of topically applied ketorolac tromethamine in vitro and in vivo," Int. J. Pharm. 570(118641 (2019).

5. L. M. Levine, "Basic and Clinical Science Course, Section 2: Fundamentals and Principles of Ophthalmology," in Basic and Clinical Science Course, p. 430, American Academy of Ophthalmology, (2018-2019).

6. C. C. Pelletier, J. L. Lambert, and M. Borchert, "Determination of glucose in human aqueous humor using Raman spectroscopy and designed-solution calibration," Appl. Spectrosc. 59(8), 1024-1031 (2005).

7. J. J. Vos, and D. van Norren, "Retinal damage by optical radiation. An alternative to current, ACGIHinspired guidelines," Clin. Exp. Optom. 88(4), 200-211 (2005).

8. P. International Commission on Non-Ionizing Radiation, "ICNIRP Guidelines on Limits of Exposure to Laser Radiation of Wavelengths between $180 \mathrm{~nm}$ and 1,000 mum," Health. Phys. 105(3), 271-295 (2013).

9. $\quad$ M. V. Schulmerich et al., "Dark field Raman microscopy," Anal. Chem. 82(14), 6273-6280 (2010).

10. S. Zhang et al., "Dark-field illumination in conjunction with confocal Raman spectroscopy for real-time noninvasive aqueous humor investigation," Opt. Eng. 59(09), (2020).

11. C. J. F. Bertens et al., "Pipeline for the removal of hardware related artifacts and background noise for Raman spectroscopy," MethodsX 7(100883 (2020).

12. M. Elshout et al., "Detection of Raman spectra in ocular drugs for potential in vivo application of Raman spectroscopy," J. Ocul. Pharmacol. Ther. 27(5), 445-451 (2011).

13. F. J. H. Douglas A. Skoog, Stanley R. Crouch, Principles of Instrumental Analysis, 7th ed., Cengage Learning (2016).

14. H. L. Liou, and N. A. Brennan, "Anatomically accurate, finite model eye for optical modeling,".J Opt. Soc. Am. A. Opt. Image. Sci. Vis. 14(8), 1684-1695 (1997).

15. C. J. F. Bertens et al., "Topical drug delivery devices: A review," Exp. Eye Res. 168(149-160 (2018). 


\section{Chapter 7}

Harnessing abruptly auto-defocusing beam to enhance the Raman signal in aqueous humor: a simulation analysis

Shuo Zhang", Shuhe Zhang ${ }^{\#}$ and Tos T.J.M. Berendschot \# Equal contributing Optics Communications 2021:496:127125 DOI: 10.1016/j.optcom.2021.127125 


\begin{abstract}
Raman aqueous humor detection provides a non-invasive, molecular-level approach for ingredient analysis within aqueous humor. However, current Raman aqueous humor applications are facing low signal-to-noise levels due to the trade-off between laser power and laser safety. In order to increase Raman signal while guaranteeing laser safety, in the research, we propose to use the abruptly auto-defocusing (AADF) beam as the illumination source for Raman aqueous humor spectroscopy. The ray-tracing sketch together with the propagation simulation of AADF shows its evolution within the aqueous humor. The intensity distributions are analyzed with and without the impact of corneal refraction. Results show that the efficiency of AADF is higher than the conventional focused Gaussian beam (FGB) method with center blocked. The peak value of the Raman signal intensity acquired using the AADF method is about 6 times larger than that of an FGB.
\end{abstract}

\title{
Key Words
}

Raman spectroscopy, Aqueous humor, Abruptly auto-defocusing beam 


\section{Introduction}

The aqueous humor $(\mathrm{AH})$ is a biofluid in the ocular system that provides nutrition to the anterior segment of the eye and removes metabolic wastes from the tissues herein. It is located in the anterior eye chamber, bordered by the cornea, the iris diaphragm, and the pupil, and contains mainly water and some biomolecules. ${ }^{1}$ Imbalance of the molecular composition of these biomolecules can be indicative of systematic disease or dysfunction of the visual system. Hence, molecular profiling of the AH can reveal crucial information about these physiological and pathological changes.

There are several analytical methods to investigate the molecular composition of the $\mathrm{AH}{ }^{2}$ For example, liquid chromatography (LC) can quantify $\mathrm{AH}$ samples with minimal sample pre-treatment. Its detection limit is able to reach the $\mathrm{ng} / \mathrm{mL}$ level. ${ }^{3}$ The mass spectroscopy shares the same advantages on the detection limit as that of LC but is easier to use and less expensive to deploy and operate than the LC. ${ }^{4}$ The biggest shortcoming of these methods is that they need invasive biopsy sampling, and can increase the potential health risks, like inflammation and pain for patients.

As a non-invasive and non-destructive spectrometry-based technique, the applications of Raman spectroscopy in the identification and quantification of biomolecules enabled its role in variant biomedical applications in ophthalmology. ${ }^{5}$ Erckens et al. demonstrated the possibility of the Raman technique for identifying three materials of poly methylmethacrylate (PMMA), acrylic, and silicone as an intraocular lens in a patient. ${ }^{6}$ They have also demonstrated the in-vivo evaluation of drug-induced cornea hydration changing by continuous monitoring water content in rabbit eyes. ${ }^{7}$ Hosseini quantified the local concentration of intraocular drugs used for endophthalmitis by Raman spectroscopy. ${ }^{8}$ Bauer et al. investigated ocular pharmacokinetics in rabbit eyes by a confocal Raman spectroscopy system. ${ }^{9}$

Bedside its applications in detecting ocular drugs and water content, Raman spectroscopy can also be employed to detect particular molecules and to discriminate between normal and diseased states. For example, Kaji et al. showed the capability of visualizing and localizing collagen fibers, proteins, lipids, and DNA/RNA in the rat cornea by coherent anti-Stokes Raman scattering. ${ }^{10}$ Paluszkiewicz et al. reported the Raman spectrum from two human lenses after cataract surgery. They found an excess of tryptophan, tyrosine, phenylalanine, Beta-sheet, the cause being still under discussion. ${ }^{11}$ In comparing healthy and cataractous human lenses with Raman spectroscopy, they found differences in the concentration of Tyr and Trp residues. ${ }^{12}$

Erckens et al. reviewed Raman spectroscopy applications in ophthalmology ${ }^{13}$ and showed that it can be used for the purpose of aqueous humor detection. ${ }^{14}$ This idea was further substantiated by Lambert et al., ${ }^{15}$ in which the glucose concentration in the $\mathrm{AH}$ is detected by Raman spectroscopy for diabetes diagnosing. Bertens and Zhang et al. explored the feasibility of Raman spectroscopy to measure ocular drug concentrations in living animals. $^{3}$ 

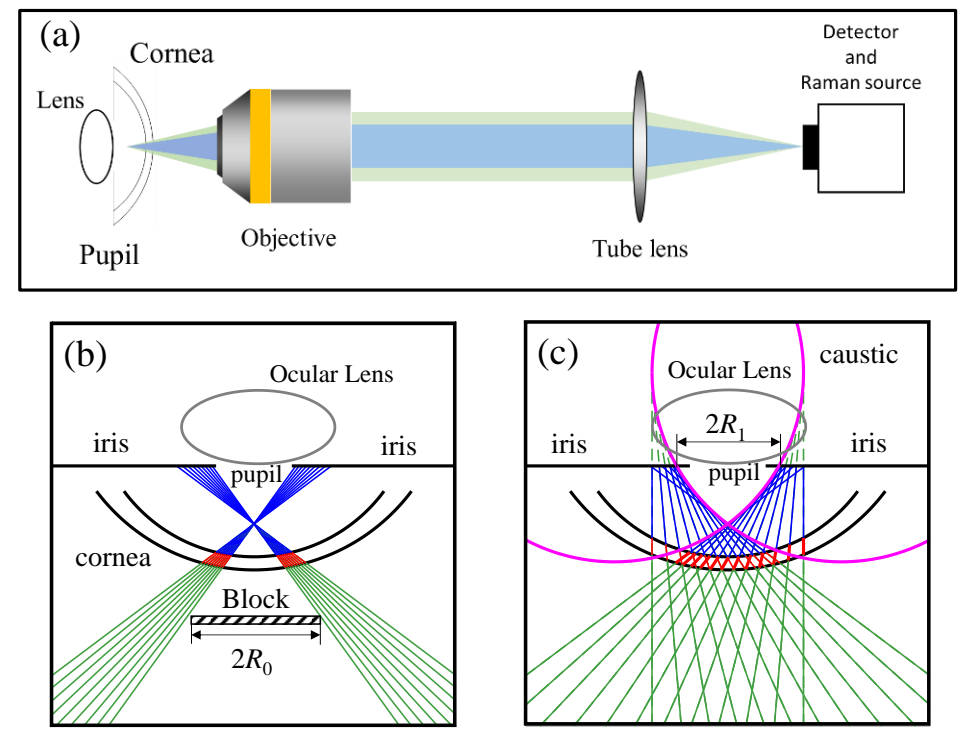

Fig. 1. Sketch of traditional aqueous humor Raman. (a) confocal humor Raman. (b) blocked focused Gaussian beam. (c) Concept of using AADF.

The applications of Raman spectroscopy on animal eyes are promising, but its applications on human eyes are facing a big challenge-the trade-off between laser safety and high Raman signal. A traditional aqueous humor Raman spectroscopy setup can be regarded as a confocal system, as shown in Fig. 1(a). Laser light, which can be regarded as a collimated Gaussian beam, is focused by an objective lens into the chamber between the cornea and ocular lens. The $\mathrm{AH}$ herein is stimulated, producing back-scattered Raman signals to be measured by a detector. The Raman signal strength is proportional to the intensity of stimulating laser light. However, the focusing laser will pass through and be focused by the ocular lens, then further arrives at the retina, which is highly sensitive for light damage. In order to prevent the retina from being burned by laser, Zhang et al. proposed two designs with a dark-illumination approach and obtained ocular drug concentrations in animal eyes ex vivo. ${ }^{16,17}$ An alternative solution is to place a black screen between the eye and objective lens or within the light pathway to block out part of the beam that will enter the pupil. Figure 1(b) shows a ray-tracing sketch of this idea.

Unfortunately, such a screen will also block the back-scattered Raman signal, leading to a low signal-to-noise ratio. Hence the key issue to be addressed is how to enhance the Raman signal while preventing light damage in the retina. In other words, we need a beam that can be focused with a high-intensity spot at the focal point, with a decreased intensity thereafter. The aim of this paper is to propose an abruptly auto-defocusing beam to meet these requirements. 
The abruptly auto-defocusing field (AADF) is an inverse propagating version of the abruptly auto-focusing beam (AAF). ${ }^{18,}{ }^{19}$ Figure $1(\mathrm{c})$ shows a ray-tracing sketch using AADF for Raman spectroscopy in the AH. With the propagating of the AADF, the beams' caustic surfaces become closer and closer and collapse at the cross-point of the caustic surfaces. ${ }^{19}$ Optical waves interfere intensively at that point creating a high-intensity focal spot. After the focal spot, the beam's caustic surfaces begin to separate, resulting in an abrupt decrease of intensity after the beam's focal point and producing an intensity-empty area between the separated caustic surfaces. Considering that the caustic surfaces of an AADF beam can be pre-engineered using ray-optics, ${ }^{19-23}$ it is possible to design a special AADF beam in which the caustic surfaces have already separated enough at the pupil after it collapses at the chamber between the cornea and iris, such that no optical field will arrive at the retina.

\section{Abruptly auto-defocusing field}

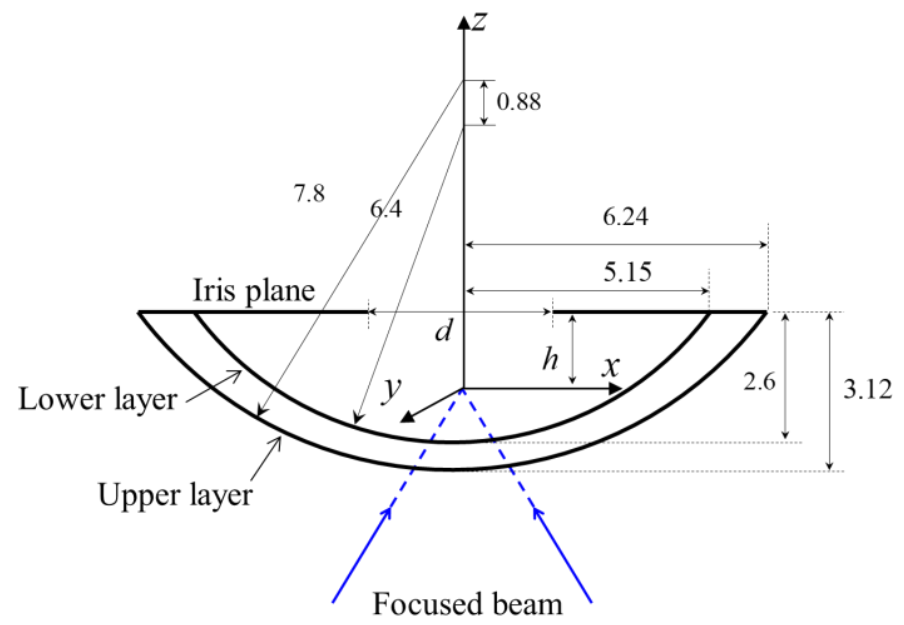

Fig. 2

Fig. 2. Sketch for beam focusing in aqueous humor. The geometry for the cornea surface and the iris are adopted from the Liou-Brennan eye model.

Figure 2 shows the Liou-Brennan eye model that we used in the following simulations. ${ }^{24}$ It contains two spherical surfaces for the cornea, with a curvature of $7.8 \mathrm{~mm}$ for the outer layer and $6.4 \mathrm{~mm}$ for the inner layer. The center of the two spherical surfaces located on the $z$-axis, with a distance of $0.88 \mathrm{~mm}$. The distance between the iris plane and the vertex of the inner layer is $2.6 \mathrm{~mm}$.

For Raman spectroscopy, a laser beam needs to be focused inside the anterior eye chamber. Let's consider an optical beam focused on an objective lens with a given numerical aperture $(N A)$. The distance from the geometrical focal point of the objective lens to the iris plane is $h$. The geometrical focal point denotes a single cross point of light rays 
when focused by the lens, which is corresponding to the position of the focal spot of a focused Gaussian beam (FGB), as well as the auto-defocusing point of an AADF. Due to the diffraction of optical waves, the optical focal spot is located near the geometrical focal point.

According to the rotational symmetric of the eye model and both of the Gaussian beam and the AADF, we used a $3 \mathrm{D}$ cylindrical coordinates system $(r, \theta, z)$ to convert the $3 \mathrm{D}$ problem into a $2 \mathrm{D}$ situation, where the origin was placed at the geometrical focal point as shown in Fig. $2, r^{2}=x^{2}+y^{2}$, and $\varphi=\arctan (y / x)$.

Due to the Fourier transform property of the objective lens, the output optical field, which propagates along the positive $z$-axis at any given observation point $(r, \theta, z)$ is given by the superposition of the plane waves weighted by their angular spectrum ${ }^{25,26}$ :

$$
U(r, \varphi, z)=\int_{\theta_{\min }}^{\theta_{\max }} A_{0}(\theta) J_{0}(k r \sin \theta) \exp (i k z \cos \theta) \exp [i \Phi(\theta)] \sin \theta \mathrm{d} \theta
$$

where $k=2 \pi / \lambda$ and $\lambda$ is the wavelength. $\theta$ is the angle between the direction of plane waves and the positive $z$-axis. $A_{0}(\theta)$ is a real function that weights different components of the plane waves. $J_{0}$ is the zero-order Bessel function of the first kind. $\Phi(\theta)$ is also a real function that denotes the phase difference of each plane wave. Different types of optical fields can be generated by adjusting $\Phi(\theta)$. For an FGB $\Phi(\theta)=0$, and for an AADF with circular caustics $\Phi(\theta)$ is given by ${ }^{27}$ :

$$
\Phi(\theta)=-k f\left(\theta \csc \theta_{\text {max }}-\sin \theta \cot \theta_{\text {max }}+\cos \theta\right)
$$

Here $f$ is the parameter that controls the length of the position of the focal point of AADF. The half of the distance between the two caustics at the iris plane is given by ${ }^{27}$

$$
R_{1}=f \csc \left(\theta_{\text {max }}\right) \cdot \cos \left\{\arcsin \left[\frac{f-h}{f \csc \left(\theta_{\text {max }}\right)}\right]\right\}-f \cot \left(\theta_{\text {max }}\right)
$$

$R_{1}$ should not be smaller than the radius of the pupil. The optical aberration due to the corneal refraction is ignored since it can be compensated by adding an additional phase term to $\Phi(\theta)$. The upper limit of the integration $\theta_{\max }$ in Eq. (1) is given as $\theta_{\max }=\arcsin (N A)$. The lower limit of integration, $\theta_{\min }=0$ for the AADF. While for FGB, $\theta_{\min }$ is determined by the block length and $h$ due to the impact of the block. Let $R_{0}$ be the half of the block length and $d$ be the diameter of the pupil. We have $R_{0}>d / 2 . \theta_{\text {min }}$ can be calculated by $\theta_{\min }=\arctan \left(R_{0} / h\right)$. According to Eq. (1), the $N A$ of the objective lens must satisfy $N A>\sin \left(\theta_{\min }\right)$ to ensure the illumination intensity is not zero for the case of FGB. 
When the beam enters the anterior eye chamber, the substances at the focal spot are stimulated by the illumination field $U(r, \varphi, z)$ and will scatter Raman signal toward the objective lens. Raman scattering is an inelastic scattering and an incoherent process, in which photons are scattered in random directions ${ }^{28}$. The Raman detector is a barrel detector that detects the total intensity collected by the objective lens. For simplicity, we assume that the total intensity of the Raman signal collected by the objective lens from a stimulated point $\left(r_{0}, \varphi_{0}, z_{0}\right)$ is proportional to the product of the illumination intensity $\left|U\left(r_{0}, \varphi_{0}, z_{0}\right)\right|^{2}$. The objective lens collects all signals within the focal spot. Since the intensity of the focal spot reaches its peak in the $z$-axis, we consider only the signal emitted from a single point at the peak of the intensity. The total Raman signal that is collected by the objective lens can thus be calculated using

$$
I_{R} \sim|U(r=0, \varphi=0, z)|_{\max }^{2} \int_{\Omega} \mathrm{d} \Omega=2 \pi|U(r=0, \varphi=0, z)|_{\max }^{2} \int_{\theta_{\min }}^{\arcsin N A} \sin \theta \mathrm{d} \theta
$$

Where $\Omega$ is the solid angle measured from point $\left(r_{0}, \varphi_{0}, z_{0}\right)$ to objective's aperture. Our purpose is to compare the value of $I_{R}$ for both FGB and AADF. Although $\Omega$ changes with the different positions of points on the $z$-axis, if we consider only the small region near the focal spot, the change of $\Omega$ can be ignored compared with the aperture diameter of the objective lens.

\section{Comparison between AADF and FGB}

In the simulation part, we assume that $h=2300 \mu \mathrm{m}, \lambda=0.785 \mu \mathrm{m}$, and the numerical aperture of objective lens $N A=0.58$. The human pupil diameter is normally around 2 to 4 $\mathrm{mm}$. However, the value of human pupil diameter in the simulation needs to be carefully chosen. On the one hand, the pupil diameter shouldn't be very small such as $2 \mathrm{~mm}, 1 \mathrm{~mm}$, or even smaller, since these cases are difficult to achieve and be stabilized in every practical experiment. On the other hand, the pupil diameter shouldn't be very large, due to the limitation of $N A$ of the objective lens - the smallest angle of inclination illumination component might exceed the $N A$. We found that $d=3 \mathrm{~mm}$ (3000 um) can be an appropriate situation for the diameter of the human pupil.

We ignored corneal aberrations since we assumed that these aberrations are already compensated for in the phase term in Eq. (1). The block length of FGB was taken just equal to $d$, where $2 R_{0}=d$. For AADF, we assumed that $2 R_{1}=d$. When the beam waist of the incident Gaussian beam matches the entrance pupil of the objective lens, $A_{0}(\theta)=\exp \left(-\sin ^{2} \theta / N A^{2}\right)$ in Eq. (1). This value is also applied to AADF so that the total energy of FGB without block is equal to that of AADF. When the block is placed, the light energy of FGB within the range of the block is wasted. 

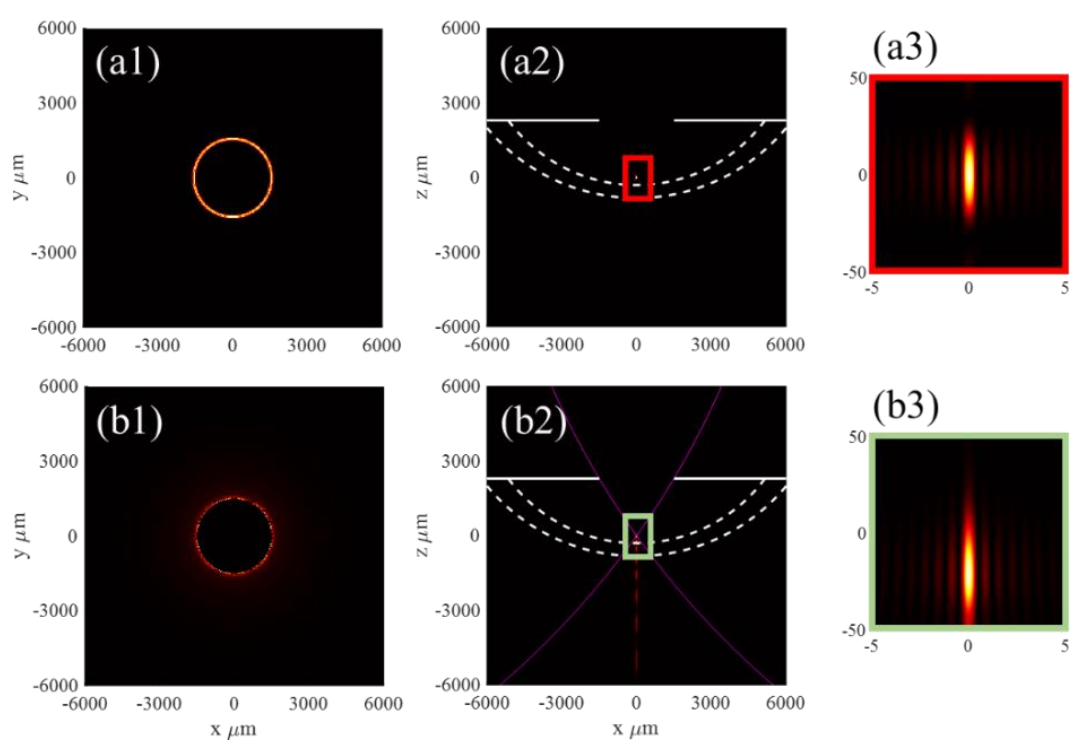

(b3)

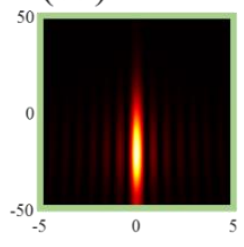

Fig. 3, 2D intensity distribution for FGB and AADF. (a1) is the transverse intensity distribution of FGB at the iris plane. (a2) is the intensity distribution at the $x o z$ plane for FGB. (b1) is the transverse intensity distribution of AADF. (b2) is the intensity distribution of AADF at the $x o z$ plane. (a3) and (b3) are enlarged parts in the red box and green box in (a2) and (b2), respectively.

Figure 3(a1) shows the normalized 2D intensity distribution of an FGB at the iris plane. Due to the block, a light ring is formed which can be also used for alignment. The diameter of the center dark circle area is larger than that of the iris to prevent retinal light damage. Figure 3(a2) shows the normalized 2D intensity distribution at the xoz-plane where $y=0$. An FGB creates a focal spot at the focal point (origin).

Fig. 3(b1) shows the normalized 2D intensity distribution at the iris plane $(z=2300$ $\mu \mathrm{m})$ for the AADF, which is similar to Fig. 3(a1). Fig. 3(b2) shows the normalized 2D intensity distribution at the $x o z$ plane for AADF. According to Fig. 3(b2), almost the entire surface of the cornea is illuminated by the AADF. Laser intensity is more concentrated at the vertex part of the cornea $(x=y=0)$. In general, the intensity on the cornea surface is rather weaker than that of the focal spot, which is approximately located at $z=-21.02 \mu \mathrm{m}$. Different from Fig. 3(a2), the AADF shows an elongated focal spot in the $z$-direction as shown in Fig. 3(b3), resulting in a weaker peak intensity than that of FGB. 

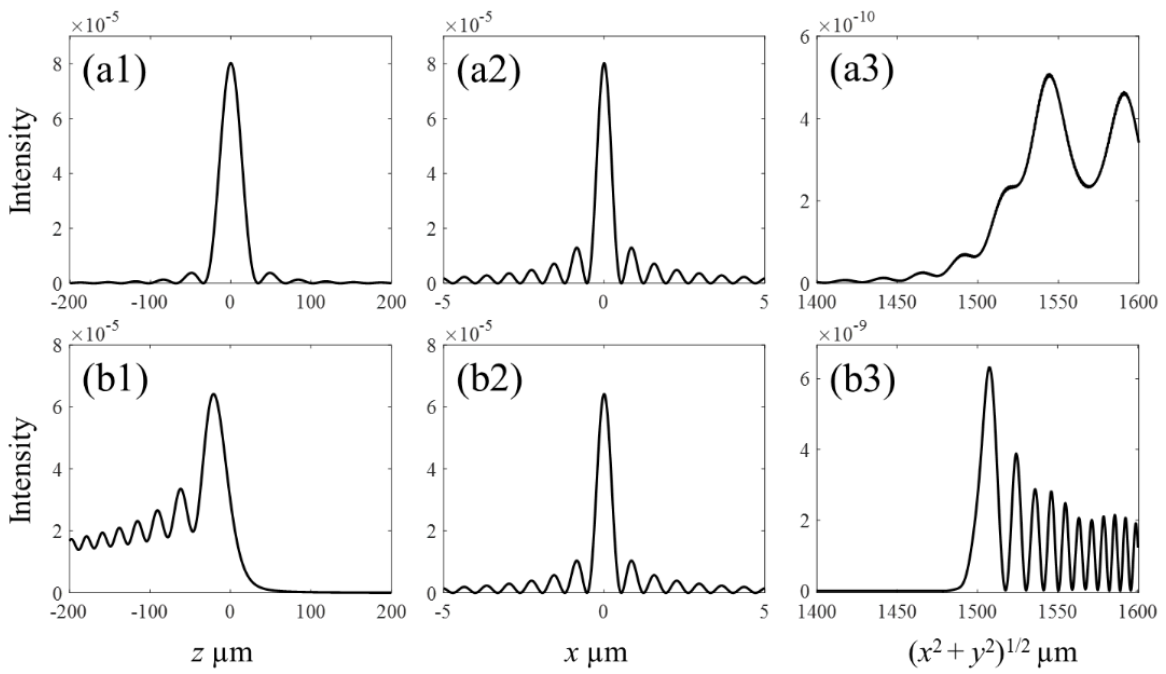

Fig. 4. Curves of intensity distribution for FGB and AADF. (a1) and (a2) are axial and transverse intensities for FGB near the focal spot. (a3) is the transverse intensity of FGB at the iris plane. (b1) and (b2) are axial and transverse intensities for AADF near the focal spot. The peak value of intensity for AADF is approximately located at $\mathrm{z}=-21.02 \mu \mathrm{m}$. (b3) is the transverse intensity of AADF at the iris plane.

More detailed intensity distributions are shown in Fig. 4(a1) to Fig. 4(a3) for FGB. Figure 4(a1) is the axial intensity distribution, which shows rapid focusing near the focal point. Figure 4(a2) is the transverse distribution of the focal spot and Fig. 4(a3) the transverse distribution at the iris plane. The axial intensity distribution for AADF, Fig. 4(b1), shows an oscillating increase and reaches its peak near the focusing point $(z=0)$. Hereafter the intensity rapidly decreases to zero with the beam propagating towards the positive $z$-axis. The transverse distribution of the focal spot is shown in Fig. 4(b2) which is similar to that in Fig. 4(a2). The transverse intensity distribution at the iris plane for AADF is shown in Fig. 4(b3), showing chirping-like oscillations, denoting the self-accelerating feature. $^{22,29,30}$

Comparing Fig. 4(a1) to Fig. 4(b1), the peak intensity of AADF is not as high as that of FGB. This can be also explained using the theory of ray optics where the light intensity is in inverse proportion to the distances of light rays. ${ }^{31,32}$ For an FGB, light rays are tightly focused at the focal point, where the distance of all light rays is zero. For an AADF, light rays are not directly focused at the focal point, but tangent to the caustic surface. Due to the collapse of the caustic surface, the optical fields interfere at the collapse point creating the bright focal spot. At the collapse point, light rays are not as tight as that of directly focusing resulting in that the focal spot intensity of AADF is not as high as the FGB.

Although the illumination intensity for FGB is larger than that of AADF, the signal intensity of FGB is weaker than AADF as shown in Fig. 5(a) due to the presence of the block. According to Eq. (4), the signal intensity not only depends on the illumination 
intensity but also relies on the solid angle of $\Omega$, within which the signal can be collected by the objective lens. For AADF, $\Omega \approx 0.37 \pi$ which can be directly calculated using the $N A$ of the objective lens. While $\Omega \approx 0.046 \pi$ for FGB. Due to the block, a large part of Raman signals is removed and cannot be received by the objective lens. We also simulated the performance of AADF and FGB under different wavelengths and $N A$. Let $I_{R 1}$ and $I_{R 2}$ be the intensity of the Raman signal for AADF and FGB, respectively. With $N A=0.58$ for the illumination beam, the ratio of Raman $\left(I_{\mathrm{R} 1} / I_{\mathrm{R} 2}\right)$ signal between AADF and FGB increases with increasing wavelengths as shown in Fig. 5(b). Moreover, the signal strength of AADF is at least 5 times larger than that of FGB. This is due to the property of AADF of which the peak intensity gets larger with the increase of wavelength as shown in Fig. 5(d).

For fixed $\lambda, I_{\mathrm{R} 1} / I_{\mathrm{R} 2}$ decreases with increasing $N A$. This can be explained as follows. First, the increase of $N A$ will enhance the peak intensity of both AADF and FGB, however, FGB is focused more tightly than AADF, with its peak intensity also increasing faster than that of AADF as shown in Fig. 5(e), resulting in the decrease of $I_{\mathrm{R} 1} / I_{\mathrm{R} 2}$. Second, the increase of $N A$ also enlarges the range of the solid angle that can be collected by the objective lens.

Figure 5(f) shows the change of $I_{\mathrm{R} 1} / I_{\mathrm{R} 2}$ with respect to $N A$ and $\lambda$. In general, the AADF will have better performance than FGB with a larger illumination wavelength and small $N A$. In addition, Raman signal efficiency of two alternative FGB configurations are discussed in the Appendix section.
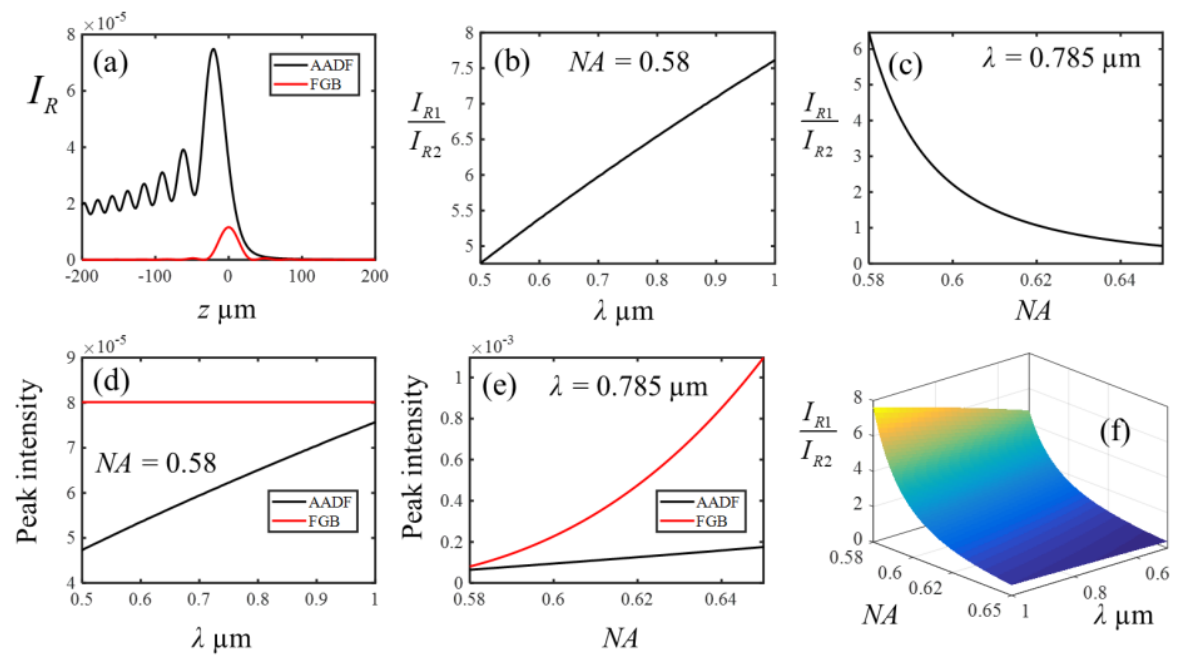

Fig. 5. Comparison of strength of Raman signal for AADF and FGB. (a) axial Raman signal for AADF and FGB. (b) and (c) are the ratio of Raman signal of AADF and FGB with different $N A$ and $\lambda$. (d) and (e) are changes of the peak intensity of AADF and FGB with different $N A$ and $\lambda$. (f) are $I_{\mathrm{R} 1} / I_{\mathrm{R} 2}$ with respect to $N A$ and $\lambda$. 


\section{Impact of the corneal refraction}

In the above simulation, we ignored the impact of corneal aberration since we assumed them to be compensated in the phase term of Eq. (1). Corneal aberrations can be rather complex for individual subjects due to the difference of corneal curvature, thickness, and the mismatch of refractive index between cornea and air and cornea and anterior eye chamber. The combination of those intricate situations will make it hard to simulate the impact of corneal aberration.

However, we can simplify the analyses by considering only the case of mismatch of the refractive index, that is the impact of corneal refraction. This is a reasonable assumption due to the following reasons. First, the optical field of the incident beam at the front surface of the cornea mainly locates in a central circular area of the cornea due to the focusing properties of the incident beam. The radius of the circular is approximately equal to $1 \mathrm{~mm}$. This can be also demonstrated through the ray-tracing method as shown in Fig. 7(a). Second, optical aberrations for the central part of the cornea can be ignored.
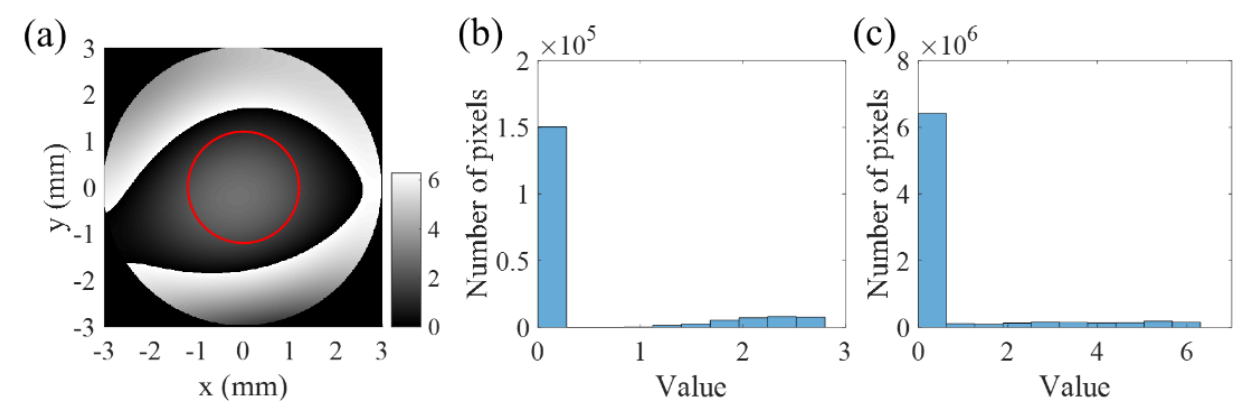

Fig. 6 distribution of corneal aberrations. (a) aberrations for one subject. (b) and (c) are statistic distribution of corneal aberration for (b) and all 42 subjects in the red circle area.

We verified this property by measuring corneal aberrations in 42 subjects using a Pantacam setup, a typical example of which is shown in Fig. 6(a) (512 by 512 pixels). Here the phase distribution is moded by $2 \pi$. The Pentacam measures corneal aberrations of a circular area with a diameter of $6 \mathrm{~mm}$. The red circle in Fig. 6(a) with a radius of $1.2 \mathrm{~mm}$ denotes the circular area of the incident beam. Figure 6(b) shows the statistical distribution of corneal aberrations within the red circle in Fig. 6(a). Accordingly, the aberrations were mainly close to zero, meaning that the aberrations within the red circle can be regarded as uniformly distributed. The statistic distribution of corneal aberration for all 42 subjects is shown in Fig. 6(c). The result shows a similar distribution to that in Fig. 6(b).

The impact of the corneal refraction is demonstrated through ray-tracing as shown in Fig. 7(a1) for FGB and 7(b1) for AADF. Here we assumed 1.38 for the refractive index of the cornea and 1.33 for the anterior eye chamber. ${ }^{33}$ The blocked range, $R_{0}$, for FGB and the parameter $R_{1}$ of AADF remained unchanged as in section 4 , where $R_{0}=R_{1}=1500 \mu \mathrm{m}$. Further, $h=2300 \mu \mathrm{m}$. $\lambda=0.785 \mu \mathrm{m}$ and $N A=0.58$ for the objective lens. The direction of 
light rays can be determined using the geometrical relation that light rays are tangent to the caustics. ${ }^{31}$ The direction of spatially refracted light rays can be calculated using the quaternion-rotation method ${ }^{34}$ so that the change of optical path length can be numerically calculated according to ray-tracing, which further changes the value of the phase term $\Phi(\theta)$ in Eq. (1).

Black dashed lines in Fig. 7(a1) denote the light rays that are blocked. Purple curves in Fig. 7(a2) are the caustics of AADF without the impact of aberrations. Due to the mismatch of the refractive index, the focal point is shifted about $321 \mu \mathrm{m}$ away toward the positive $z$ axis. The diameter of the pupil should be further reduced to $2000 \mu \mathrm{m}$ in order to prevent the retina from being damaged by light beams. The 2D intensity distributions for FGB and AADF are shown in Fig. 7(a2) and 7(b2). Figures 7(a3) and 7(b3) show $I_{\mathrm{R}}$ along the $z$-axis of 7(a2) and 7(b2). The peak value of $I_{\mathrm{R}}$ for AADF (7(b3)) is about 6 times larger than that of FGB (7(a3)).

Although the position of the focal spot is shifted due to the mismatch of the refractive index, the profile of the intensity distribution hardly changes. If we ignore the change of refractive index with respect to wavelength, the relationship between peak $I_{\mathrm{R}}, \lambda$, and $N A$ is similar to the result in section 4, i.e. the AADF has better performance than FGB with a larger illumination wavelength and small NA. Moreover, according to Figs. 7(b1) and 7(b3), the AADF still shows its abruptly defocusing property and can be used to avoid excitation light beams entering the pupil. Therefore, only Rayleigh or Mie scattered photons might reach the posterior section of the eye through the crystalline lens which is within the safe limit. ${ }^{35}$ Compared with the strength of the Raman signal of FGB, the AADF is still able to enhance the signal strength up to 6 times.
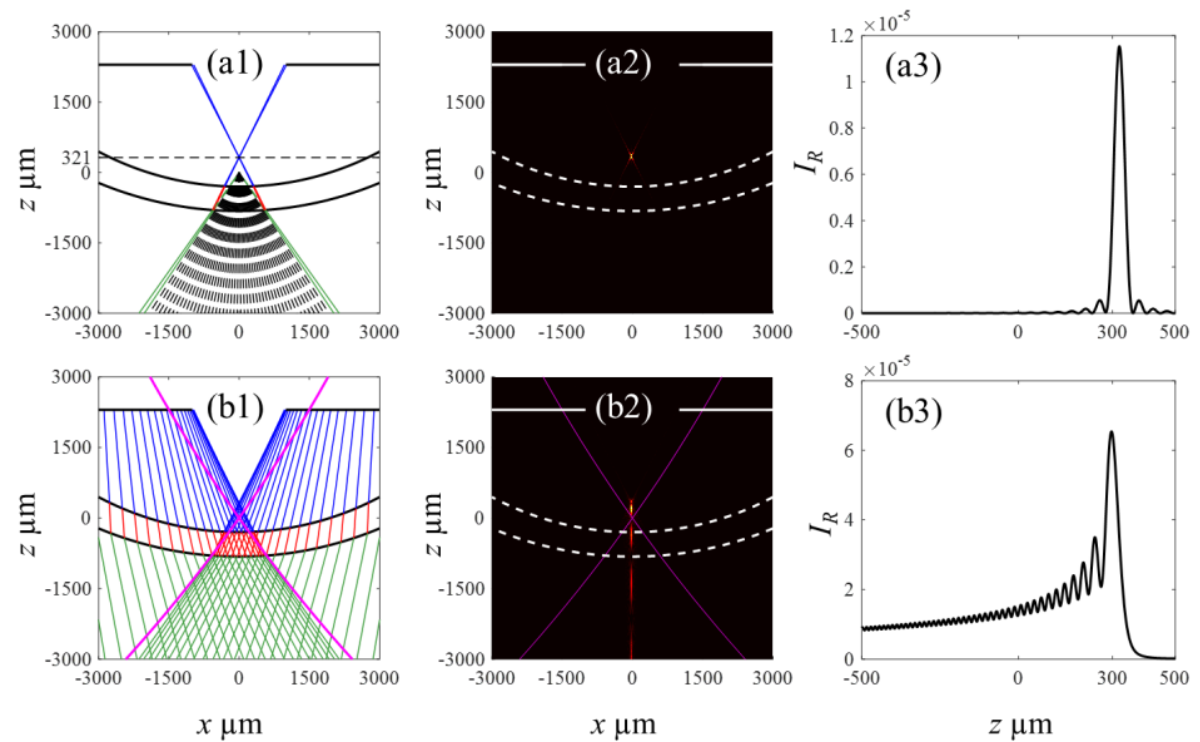
Fig. 7. Performance of AADF and FGB under the impact of mismatch of refractive index. (a1) and (b1) are ray tracing sketches for FGB and AADF. (a2) and (b2) are the 2D intensity distribution in xoz plane. (a3) and (b3) are the detailed axial Raman signal in the $z$-axis for FGB and AADF. The black dashed lines in (a1) denote the blocked light rays. Purple curves in (b1) are the designed caustic for AADF without the impact of aberration.

\section{Concluding remarks}

In this study, we propose to use an abruptly auto-defocusing (AADF) beam to increase the Raman spectroscopy of the anterior eye chamber. Taking the advantage of the autodefocusing feature of the AADF, a focal spot of high intensity is created inside of the anterior eye chamber. After that, the beam automatically defocuses along its caustic surface yielding an intensity-free circular area at the pupil plane, preventing the retina from being burnt by laser beams.

The performance of the AADF is analyzed theoretically and compared with conventional focused Gaussian beam (FGB) approaches. The simulations are performed without and with the impact of the corneal refraction. The influence of wavelength and NA of the objective lens are also simulated. The results show that AADF has better performance than FGB, where the AADF produces a focal spot with about 6-fold higher intensity than that of a center-blocked FGB, which will largely increase the useful Raman signal for detection.

In a practical experiment, the AADF won't lead to additional complexity of optical aligning between the two centers of the pupil and the beam's dark circular area than that of FGB. Both two methods face optical aligning, and unintended eye motion problems. The light rings on the pupil plane might be of help for the alignment procedure. In addition, the impact of irregular corneal shapes like keratoconus or cloudy cornea like keratitis also reduces the performance of traditional FGB and the proposed AADF methods. At the current stage, the main challenge of applying AADF to the aqueous humor Raman detection remains on how to produce such an optical field after the objective lens. Using a spatial light modulator or a phase mask could be good solutions.

\section{Acknowledgments}

Shuo Zhang and Shuhe Zhang acknowledge the China Scholarship Council for the support by State Scholarship Fund No. 201309110103 and No.201908340078, respectively.

\section{Conflicts of interest}

The authors declare no conflict of interest. 


\section{References}

1. L. M. Levine, "Basic and Clinical Science Course, Section 2: Fundamentals and Principles of Ophthalmology," in Basic and Clinical Science Course, p. 430, American Academy of Ophthalmology, (2018-2019).

2. K. Pietrowska et al., "Analysis of pharmaceuticals and small molecules in aqueous humor," $J$ Pharm Biomed Anal 159(23-36 (2018).

3. C. J. F. Bertens et al., "Confocal Raman spectroscopy: Evaluation of a non-invasive technique for the detection of topically applied ketorolac tromethamine in vitro and in vivo," Int. J. Pharm. 570(118641 (2019).

4. B. Caballero, L. Trugo, and P. Finglas, "Caballero, Benjamin, in:Encyclopedia of food sciences and nutrition," in Encyclopedia of food sciences and nutrition: Volumes 1-10., pp. 3739-3749, Academic Press,, Oxford, (2003).

5. R. J. Erckens et al., "Raman spectroscopy in ophthalmology: From experimental tool to applications in vivo," Lasers Med. Sci. 16(4), 236-252 (2001).

6. R. J. Erckens et al., "Noninvasive Raman spectroscopic identification of intraocular lens material in the living human eye," J. Cataract. Refract. Surg. 27(7), 1065-1070 (2001).

7. R. J. Erckens et al., "Drug-induced corneal hydration changes monitoredin vivo by non-invasive confocal Raman spectroscopy," Journal of Raman Spectroscopy 32(9), 733-737 (2001).

8. K. Hosseini, "Optical Biopsy in the Anterior Segment of the Eye," in Ophthalmology, p. 143, Maastricht University (2008).

9. N. J. Bauer, "Potential application of confocal raman spectroscopy in ophthalmology," in ophthalmology, p. 160, Maastricht university (1999).

10. Y. Kaji et al., "Raman microscopy: a noninvasive method to visualize the localizations of biomolecules in the cornea," Cornea 36 Suppl 1(0), S67-S71 (2017).

11. C. Paluszkiewicz et al., "Analysis of human lenses by Raman microspectroscopy," Acta Physica Polonica A 129(2), 244-246 (2016).

12. C. Paluszkiewicz et al., "Vibrational microspectroscopy analysis of human lenses," Spectrochim Acta A Mol Biomol Spectrosc 188(332-337 (2018).

13. R. Erckens et al., "Raman spectroscopy in ophthalmology: from experimental tool to applications in vivo," Lasers Med. Sci. 16(4), 236-252 (2001).

14. M. J. Baker et al., "Developing and understanding biofluid vibrational spectroscopy: a critical review," Chem. Soc. Rev. 45(7), 1803-1818 (2016).

15. J. L. Lambert, C. C. Pelletier, and M. Borchert, "Glucose determination in human aqueous humor with Raman spectroscopy," J. Biomed. Opt. 10(3), 031110 (2005).

16. S. Zhang et al., "Dark-field illumination in conjunction with confocal Raman spectroscopy for real-time noninvasive aqueous humor investigation," Opt. Eng. 59(09), (2020).

17. R. J. E. Shuo Zhang, Frans HM Jongsma, Carroll AB Webers and Tos TJM Berendschot, "Design and performance of a dark field probe with confocal-Raman spectroscopy for ophthalmic applications," Journal of Raman Spectroscopy In press $((2021)$.

18. N. K. Efremidis, and D. N. Christodoulides, "Abruptly autofocusing waves," Opt. Lett. 35(23), 40454047 (2010).

19. I. D. Chremmos et al., "Abruptly autofocusing and autodefocusing optical beams with arbitrary caustics," Phys. Rev. A 85(023828 (2012).

20. I. Chremmos, N. K. Efremidis, and D. N. Christodoulides, "Pre-engineered abruptly autofocusing beams," Opt. Lett. 36(1890-1892 (2011).

21. D. G. Papazoglou et al., "Observation of abruptly autofocusing waves," Opt. Lett. 36(10), 1842-1844 (2011).

22. I. Epstein, and A. Arie, "Arbitrary Bending Plasmonic Light Waves," Phys. Rev. Lett. 112(023903 (2014).

23. R.-S. Penciu, V. Paltoglou, and N. K. Efremidis, "Closed-form expressions for nonparaxial accelerating beams with pre-engineered trajectories," Opt. Lett. 40(7), 1444-1447 (2015).

24. H. L. Liou, and N. A. Brennan, "Anatomically accurate, finite model eye for optical modeling,".J Opt. Soc. Am. A. Opt. Image. Sci. Vis. 14(8), 1684-1695 (1997).

25. E. Wolf, "Electromagnetic Diffraction in Optical Systems. I. An Integral Representation of the Image Field," Proc. R. Soc. Lond. A 253(349-357 (1959).

26. M. Born, and E. Wolf, Principles of Optics, Cambridge University Press (1999). 
27. S. H. Zhang, J. H. Zhou, and M.-C. Zhong, "Nonparaxial structured vectorial abruptly autofocusing beam," Opt. Lett. 44(11), 2843-2846 (2019).

28. R. R. Jones et al., "Raman Techniques: Fundamentals and Frontiers," Nanoscale Res Lett 14(1), 231 (2019).

29. I. Kaminer, M. Segev, and D. N. Christodoulides, "Self-Accelerating Self-Trapped Optical Beams," Phys. Rev. Lett. 106(213903 (2011).

30. M. A. Alonso, and M. A. Bandres, "Spherical fields as nonparaxial accelerating waves," Opt. Lett. 37(24), 5175-5177 (2012).

31. D. A. McNamara, C. W. I. Pistorius, and J. A. G. Malherbe, Introduction to the Uniform Geometrical Theory of Diffraction, ARTECH HOUSE (1990).

32. G. W. Forbes, and M. A. Alonso, "Using rays better. I. Theory for smoothly varying media," J. Opt. Soc. Am. A 18(5), 1132-1145 (2001).

33. K.-H. D. Michael Kaschke, Michael Stefan Rill, "Optical Devices in Ophthalmology and Optometry: Technology, Design Principles and Clinical Applications," p. 42, Wiley, Weinheeim, Germany (2014).

34. S. H. Zhang, Z. Liang, and J. H. Zhou, "Using quaternions to analyze the trapping force of an ellipsoidal bead," Acta Phys. Sin. 66(4), 048701 (2017).

35. P. International Commission on Non-Ionizing Radiation, "ICNIRP Guidelines on Limits of Exposure to Laser Radiation of Wavelengths between $180 \mathrm{~nm}$ and 1,000 mum," Health Phys 105(3), 271-295 (2013).

\section{Appendix: Signal efficiency for Raman system with a beam splitter or apertured mirror}

In order to get rid of blocking on the detection path, we used the two optical layouts shown in Fig. A1. In this appendix, we briefly analyse their signal efficiencies, advantages and disadvantages according to numerical simulations and experimental experiences.

In the first layout in Fig. A1 (a), a beam splitter is used to combine the detection path and illumination path into the same optical path. In this case, the beam splitter will reduce the light intensity. For example, the beam splitter has 50\% penetration and $50 \%$ reflection. Thus, the intensity of the detection light is actually decreased by $75 \%$. In this case, the signal collection angle is the same as that of AADF, but the light intensity is multiplied by 0.25 . The simulation result is shown in Fig. A1 (d) in the red curve. The signal intensity is larger than that of blocked FGB but is still much weaker than that of AADF. 

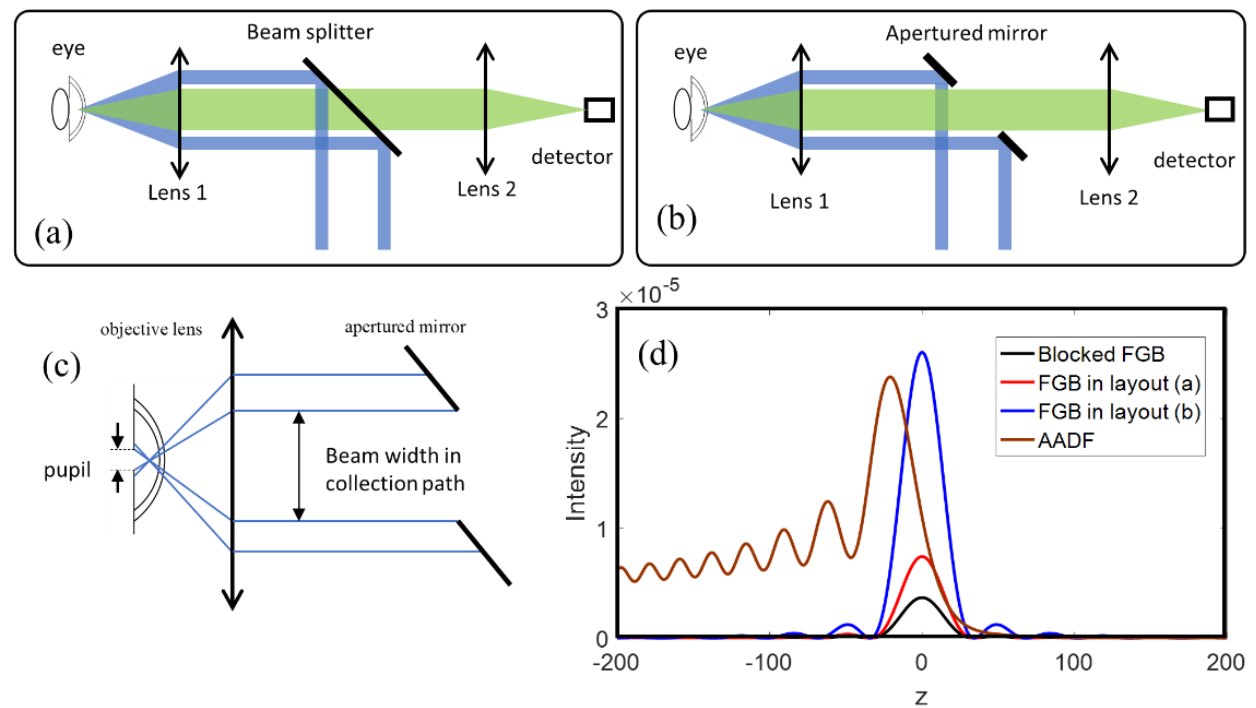

Fig. A1, Two layouts for the separation of detection and illumination path. (a) using beam splitter; (b) using an apertured mirror; (c) Geometry of collection path in layout (b); (d) Simulated Raman signal intensity in z-axis with the same beam parameters in section 3

For the second layout shown in Fig. A1 (b), the beam splitter is replaced by an apertured mirror. This layout is better than that in Fig. A1 (a) since the intensity will not be separated. However, due to the presence of the apertured mirror, the beam width in the detection path is tailored by the mirror's aperture. In an ideal situation, as shown in Fig. A1(c), the beam width in the detection path is equal to the size of the pupil's projection circle at the objective lens plane. Thus, with an upper integral limit in Eq. (4) of $\arctan [d /(2 h)]$ and a lower integral limit of 0 , the simulation results in the blue curve in Fig. A1 (d), which is slightly larger than that of AADF.

The key issue for the apertured mirror in layout Fig. A1 (b) is the alignment of the system, as misalignment can cause the excitation laser to pass through the pupil and damage the vulnerable retina. Extra efforts need to be paid for the apertured mirror alignment in its three degrees of freedom (say, the three components of its Euler angles). In addition, since the mirror must be properly installed for both the illumination path and the detection path, the difficulty in aligning is doubled compared to the direct illumination setting. 


\section{Chapter 8}

General Discussion

Shuo Zhang

Page | 131 
The aim of this thesis was to develop a dark field probe to measure concentrations of biomarker molecules in the anterior chamber of the eye using Raman spectroscopy, without compromising the retinal tissue by the strong excitation light needed to generate Raman signals.

In general, the Raman signal intensity is associated with several parameters and is given by ${ }^{1}$ :

$$
\mathrm{I}=\mathrm{CN} \ell \sigma \Omega \mathrm{F}(\mathrm{T}) \mathrm{I}_{0}
$$

Here, $\mathrm{C}$ is a constant and $\mathrm{N}$ is the number density which can be considered as concentration. The factor $\ell$ is the length of the illumination region (integration length), determined by the optical system that delivers the excitation light to the target. The influence of this factor on the Raman signal is demonstrated in chapter 1 and discussed in the first section of this chapter. The factors $\sigma$ and $\Omega$ are the Raman cross-section and scattering solid angle of the target molecule. Chapters 3-4b show how to collect acceptable Raman signals ex vivo and in vivo by properly selecting target molecules. In addition, confocal settings offer huge advantages in collecting Raman spectra from interocular tissues of interest without interference by out of focus areas. F(T) is a temperature dependent factor which can be considered as a constant since all experiment were performed in a homothermic condition. The last factor, $\mathrm{I}_{0}$, is the excitation light intensity. Due to the intrinsic weakness of the Raman effect, the use of a laser is indispensable to gain more sensitivity. However, safety concerns limit the use of a direct-illumination approach. Therefore, alternatives were developed that allow higher laser powers. They are discussed chapters 5-6 and the following sections.

\section{Raman spectroscopy applications in vitro}

Regular Raman spectroscopy has a low sensitivity and high detection limit due to the weak scattering compared to Rayleigh scattering, even when using lasers. To decrease the detection limit, both higher laser powers and longer integration times may be used. The current Raman set up has a fixed power laser, and therefore we choose to extend the exposure time. For the protein samples studied, increasing the exposure time to 60 seconds did help to pick up the characteristic peaks. However, since the Raman scattering crosssection of molecules differs, 60 s integration time is not a guarantee to obtain the characteristic peaks from some of the proteins which could be a potential biomarker. To distinguish Raman peaks from the interleukin-21 we had to use an integration time of 300s.

To obtain recognizable peaks from clinically relevant concentrations in a small volume, we further optimized the experimental setup. First, we employed a confocal setting in conjunction with two positive lenses, to exclude possible Raman signals along the light path. The laser output numerical aperture (NA) from the spectrometer is 0.05 . However, in the back-scattering Raman collection at the target, the NA of the focusing lens is 0.13 (Figure 1a). Due to the NA miss-match, not all the collection region is excited by the laser (Figure 1b). This is indicated that the intensity Raman signal could be further increased 2 
times with a NA matched optical system. In addition, not only the NA but also the imaging ratio plays a role in the effectiveness of the Raman set up. To improve this, the focusing lens could be switched to a 0.05 NA lens with an $8 \mathrm{~mm}$ diameter instead of currently using a $20 \mathrm{~mm}$ diameter lens while maintaining the same $80 \mathrm{~mm}$ FL.

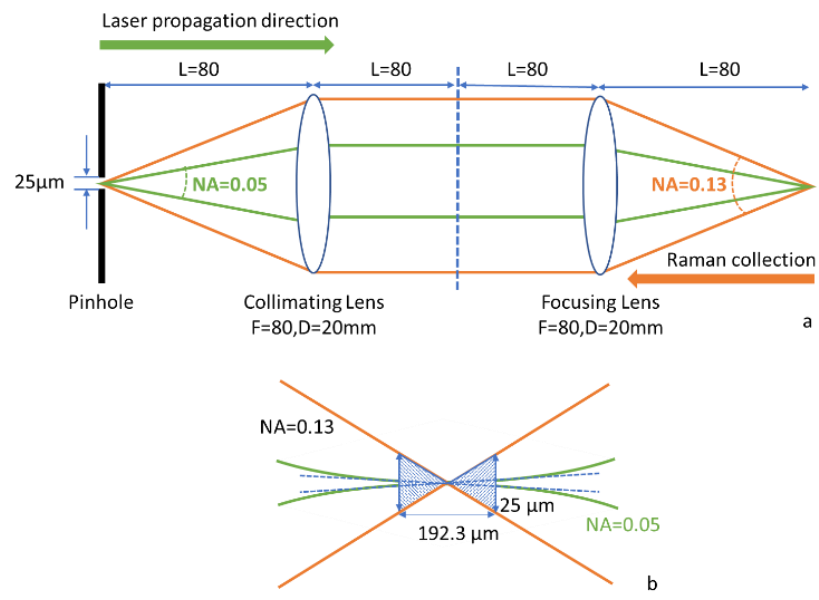

Figure 1. a) Confocal settings. B) Zoom of the focus area at the target.

Second, the protein samples were tested in their chemical form to eliminate interferences with other unknown substances that may be present in chemical complicated biological samples. The characteristic peaks from the target molecules could be identified easily with a relatively clean background.

Thirdly, the additives in the solution were all measured also individually to identify their Raman peaks for use in the background (BG) subtraction data processing. In general, there are two categories of data processing, experimentally and numerically. In most cases, both are applied jointly for background removal. However, with a controlled chemical environment and with spectra of all additives collected, the experimental approach is preferred, since the background can be assumed to consist of a combination of all the additives except the target molecules.

\section{Raman spectroscopy applications ex vivo}

Using the spatial resolution capability of confocal settings, depth profiles become available in optical transparence tissues like cornea and crystal lenses. However, two major challenges remain.

First and as mentioned previously, even if the integration time is extended the detection limit may still be too high to acquire clinically significant spectra. One of the solutions is to properly select target biomolecules with relatively strong Raman responses. For diabetic cataract we proposed Sorbitol which has a relatively large Raman scattering cross section. Using the $876 \mathrm{~cm}^{-1}$ peak, we found the detection limit by our CRS system 
could reach $2 \mathrm{mM}$, with a R-square of 0.988 in linear fitting its concentration curve. To mimic diabetic cataract development ex vivo, we immersed porcine lenses in different sorbitol concentrations. To further optimize test conditions and mimic more closely real situation, lenses could be immersed in culture media. However, the chemical environment in the lenses is already complicated enough compare to testing sorbitol solution only. By adding the culture medium, a furthermore complicated environment is created, since it consists of multiple nutrition molecules, which might significantly disturb the background spectrum.

Second, a fluorescence background is a major challenge while collecting Raman scattering signals by CRS and peaks from other compounds may also be observed. To extract the signal information of the target molecules, an experimental approach by collecting the reference spectrum from all the substance is un-realistic. Hence, a numerical approach with proper data processing technique is required to identify magnitude of the sorbitol peak in the spectra. First, the fluorescence background needs to be removed. However, currently there is no well-accepted data processing protocol. ${ }^{2}$ Polynomial fitting proved to be suitable for fluorescence background removal for the spectra collected from the lenses. The advantage of this data processing approach is that the original shapes of Raman peaks can be maintained after the treatment. Unfortunately, the optimal choice of polynomial fitting parameters varies and the performance depends on the user's

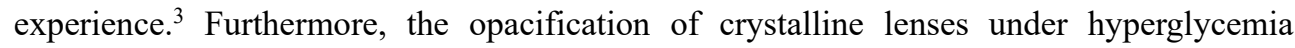
conditions overnight will affect all the Raman peaks collected from the lenses. Therefore, to quantitatively evaluate the degree of the cataract formation in the lens, ratios of peaks intensity were compared. ${ }^{4}$ The absolute value of the peaks ratios is associated with and partially influenced by the background removal process. Hence, background quantitation investigation methods selection or the parameter chosen in the background removal approach might lead to a bias result.

\section{Raman spectroscopy in vivo}

To detect molecules in vivo, also practical issues must be taken in consideration. First, we have to look at integration times. With fixed experimental settings, a longer integration time results in more Raman scattering signals collected by the CCD of the spectrometer and a better signal-to-noise ratio (SNR). However, for a contact probe (gonioscope lens) examination in clinical practice, the integration time is limited to 60 seconds. A second issue to consider is the influence of anesthetization when measuring animals. Due to individual differences, a proper delivery of the excitation light into the anterior chamber might become a challenge when animals recover early or are not fully anesthetized. Third, drug concentrations in the eye of a living animal might be lower than concentrations determined in in-vitro experiments. Pre-corneal loss of the drug (blinking, filtration of the corneal endothelium, etc.) results in a low ocular bioavailability of the drug at its target destination. Typically, less than $5 \%$ of the total drug dose administered reaches the anterior 
chamber. Hence, a lower detection limit is required. Last and as mentioned previously, the laser power is proportional to the intensity of the Raman signal. With certain biomolecules, higher laser power is preferred to gain more signal and reach a lower detection limit. However, concerns about laser safety prohibit a direct illumination approach when using high laser powers.

\section{Probe design for Raman spectroscopy}

To address the safety concerns, different dark-field probes were designed and evaluated. A first design aimed to reduce the degree of freedoms compared to a previous version, patented years ago in our group, using a three-mirror structure. Although raytracing simulation in Zemax predicted a proper functioning probe, the manufactured prototype based on this design encountered misalignment difficulties. A further ray-tracing analysis indicates a NA mismatch, non-ideal propagation distance, axial offset, mirror angular deviation and relative tilt as major contributors towards optical power loss and alignment tolerance.

To increase the SNR, in a subsequent design we removed two Aluminum ( $\mathrm{Al}$ ) coated mirrors. Since these have a reflectance of 0.873 , we expected at least $42 \%$ increase in SNR. Furthermore, the mirror deviation angle caused by misalignment was amplified two times on each mirror and by removing these two mirrors, the angle that can acquire a Raman signal hence increased four times compared to former design.

By using an artificial eye, we learned that the confocal restriction of the optical biopsy was not completed within the artificial space. This was due to the low NA of the excitation beam and the physical character of light that was not taken into account in the ray tracing. As the suppression of the Raman signal from the probe material was about 30 times, we concluded that this suppressed Raman signal, which stands for the corneal signal in a real eye, was acceptable.

To investigate in vivo molecules in the anterior eye chamber, the dark-field design paves the way to increase the laser power, since, compared to the retina, the anterior segment of the eye has a higher tolerance for light damage. By only increasing the excitation power, the detection limit could be increased two to three folds.

\section{Future perspective}

The ability to acquire intra-ocular molecular profiles in addition to structural profile will provide greater insight into ocular diseases and may result in a better diagnosis. However, further efforts are still needed to make this a reality and to have an actual device for routine in vivo measurements.

To achieve this, the main task for the future will be to improve the detection limit. A major hurdle to overcome will be to safely deliver even higher laser powers into the eye. In clinic practice, incorporating fiber optics will make it easier for ophthalmologists and may be a prerequisite for a commercial compact product. In addition, a non-contact method 
might be an alternative option to provide dark-field illumination to use Raman spectroscopy in ophthalmic applications. As we proposed in chapter 7, an abruptly autofocusing beam might be able to achieve this goal. Actual experiments need to be performed in the future to verify our simulations. In the end, machine learning and deep learning techniques could offer great help in processing the Raman spectra and unraveling the contribution of different molecules. In the not so far future, artificial intelligence will most likely be developed for data mining of spectra collected from a large number of patients for greater understanding of ocular diseases.

\section{Reference}

1. K. C. Le, C. Lefumeux, and T. Pino, "Differential Raman backscattering cross sections of black carbon nanoparticles," Sci Rep 7(1), 17124 (2017).

2. H. J. Byrne et al., "Spectral pre and post processing for infrared and Raman spectroscopy of biological tissues and cells," Chem Soc Rev 45(7), 1865-1878 (2016).

3. D. Wei, S. Chen, and Q. Liu, "Review of Fluorescence Suppression Techniques in Raman Spectroscopy," Applied Spectroscopy Reviews 50(5), 387-406 (2015).

4. C.-C. Huang, and W. Chen, "Raman spectroscopic analysis of cataract lens: A compendious review," Applied Spectroscopy Reviews 53(9), 689-702 (2018). 


\section{Chapter 9}

Summary

Shuo Zhang 
This thesis presents the use of Raman spectroscopy to investigate the ocular biomolecular status and introduces novel devices that allow high radiation energy to be delivered to the ocular system without damaging the vulnerable retinal tissue.

Chapter 1 introduces the background of this research. In current clinical practice, optical techniques are mainly used to provide anatomical, functional, and structural information. However, biochemical information is obtained by performing a biopsy of the tissue. This poses a risk to the integrity of the eye and cannot be performed on a regular base. We propose Raman spectrometry as a potential and powerful tool for a non-invasive and non-destructive investigation. The limitations of conventional Raman spectroscopy with direct illumination method in ophthalmic application are mainly safety concerns. In this thesis, biomedical applications of Raman spectroscopy are explored and a dark-field method in conjunction with confocal Raman spectroscopy (CRS) is proposed to avoid light damage of the retina.

In Chapter 2, characteristic Raman spectra of IL10and ACE in their chemical form are reported. These could be used for future in vivo diagnosis applications for eye related disease.

Chapter 3 investigated the possibilities of using Raman spectroscopy for early diabetic cataract diagnosis. Previous research indicated that the sorbitol concentration in the lens was associated with the development of diabetic cataracts. However, non-invasive quantitative assessment is still a challenge for monitoring the sorbitol concentration in the lens. We obtained the sorbitol concentration in sorbitol induced cataracts mimicking diabetic cataracts lenses in vitro. The concentration of sorbitol in pig lens shows a good correlation to the degree of the lens cataractous via their Raman spectrum.

Chapter 4 presents an ocular drug delivery study using Raman spectroscopy to determine the ketorolac concentrations in vitro porcine eyes and in vivo rabbit eyes. The porcine eyes were enucleated and soaked in different concentrations of ketorolac whereafter Raman spectra were collected by two different setups. In the first setup we used a commercial objective lens as a focusing element, and in the second a doublet lens combined with a gonioscope lens. Twelve rabbits were treated with Acular ${ }^{\mathrm{TM}}$ for four weeks, while the concentrations of ketorolac from Acular ${ }^{\mathrm{TM}}$ in aqueous humor of the rabbits were measured by CRS. All these samples were also properly collected and tested in FDA "golden standard" high-performance liquid chromatography (HPLC). The comparison of Raman spectroscopy and HPLC showed good agreement in the ex vivo experiments, but lack of sensitivity in vivo measurement.

Chapter 4a shows the raw dataset of the Raman spectrum obtained from the in vivo experiments with the rabbit eyes presented in chapter 4 . These raw datasets could provide experimental information for researchers who have no access to Raman spectra from the living rabbit eyes. The dataset might be processed by alternative data processing techniques for background suppression or characteristic peak identification of the biomarkers. The ketorolac treatments for the 12 rabbits are outlined in detail and raw Raman spectra data are presented accordingly.

Page | 138 
In Chapter $4 \mathbf{b}$, we describe the data processing procedure to minimize the hardware induced artifacts and fluorescence interference in the spectrum. A self-developed MatLab script for the automated correction of Raman spectra is presented. With the dataset in chapter $4 \mathrm{a}$, the proposed data processing method demonstrated that it can be used for minimizing the hardware and fluorescence interference simultaneously.

Chapter 5 proposed a novel device to acquire Raman spectra from the AC without jeopardizing the retina by high laser powers. In this study, the design of this dark-field device is presented. It uses a curved mirror system to guide the laser incident to the ocular system with a large angle of the optic axis of the eye. A three-dimensional computer model was built and evaluated by ray-tracing software Zemax to validate its optic performance. A prototype was manufactured and tested by AEM. The chemical compounds could be detected by the prototype in conjunction with CRS with acceptable SNR. However, the intensity of the Raman signal is still below our expectation. By means of ray tracing simulation, the alignment issue is the reason of low Raman signal collecting efficiency.

In Chapter 6, a further developed device without curved mirrors is proposed and validated in AEM. To validate, the prototype was tested by an ocular drug in AEM. The results indicated a 9 folds improvement in misalignment tolerance and a 36 folds improvement in the signal-to-noise ratio at the phenylephrine $\mathrm{HCl} 1002 \mathrm{~cm}^{-1}$ peak compared to a previous design. The prototype showed a good linear fit with the phenylephrine $\mathrm{HCl}$ concentration, with a R square of 0.996 and a sensitivity detection limit down to $0.05 \%$ phenylephrine $\mathrm{HCl}$.

Chapter 7 proposes an alternative approach to achieve a dark-field illumination method using a phase mask to generate an abruptly autofocusing beam in the anterior chamber. By modulating the phase of a circularly symmetric optical wavefront, the maximum intensity of the beams suddenly decreases by orders of magnitude right after the target point along the optic axis. In this simulation study, we demonstrate a setting that is capable of generating such a beam in the anterior chamber and create a dark-filed illumination to prevent excitation light to reach the retinal tissue directly. Results show that the efficiency of AADF is higher than the conventional focused Gaussian beam (FGB) method with center blocked. The peak value of the Raman signal intensity acquired using the AADF method is about 6 times larger than that of an FGB.

Chapter 8 discusses the major findings of this thesis regarding to using Raman spectroscopy for biomedical applications in ophthalmology. The challenges and implications for future studies both discussed in a more general and broader perspective.

In the appendix, the scientific and social impacts of the research are elaborated. The scientific impacts mainly contribute to researchers in the field of ophthalmology and physicists developing non-invasive diagnostic methods. The social impacts are aiming to benefit a wider audience outside the scientific community in various forms like clinicians, ophthalmologists, and patients eventually. Pharmaceutical industry also can be a benefit from it by reducing the animal models in drug development. 
In summary, Raman spectroscopy shows its unique potential for biomedical and preclinic applications in ophthalmology. The design of the eye probe makes one step closer to the clinic application and may serve as a groundwork for ocular biomarker non-invasive quantitative assessment for early diagnosis of ocular and systematic diseases. 
Appendix I

Impact

Shuo Zhang

Page |i 


\section{Main objective, results and conclusions}

The main objective of this dissertation was to develop a dark field probe for noninvasive determination of the molecular composition of the aqueous humor in the anterior eye chamber using Raman spectroscopy, without compromising the fragile posterior section of the eye by the strong excitation light necessary in this method. With the studies presented in this dissertation we demonstrated in vitro and in vivo in animal eyes a functional ophthalmic probe that enables to measure the concentrations of ocular drugs. The scientific and social impacts of this dissertation are highlighted in this section.

\section{Scientific impact}

The results published in peer-reviewed scientific journals and presented in (inter)national conferences contribute to researchers in the field of ophthalmology and physicists developing non-invasive diagnostic methods. Currently, clinical decision-making in ophthalmology mainly depends on the morphological screening by trained ophthalmologists. Molecular profiling could provide additional valuable information for a better understanding of the mechanics of ocular disease. Up to now however, real-time monitoring of disease progression is hampered by the fact that invasive biopsies are the only way to obtain this information. Thus, there is need for non-invasive and in-situ diagnostic methods. We propose Raman spectroscopy as a technique to be utilized for this cause. To do so, firstly spectra of target biomarkers need to be identified, as described in Chapter 2. This provides the spectra of two biomarkers related to four complex retinal diseases. Quantitative assessment of these molecules can be used as a diagnostic aid in patient care. Spectra and studies of another biomarker, sorbitol, that is generated in the pathway of diabetic cataract development in the lens, are presented in Chapter 3. This might help ophthalmologists to gain more insights into the mechanisms of early diabetic cataract development.

The feasibility of using Raman spectroscopy in drug delivery research in living animals is explored in Chapters 4 to $4 \mathbf{b}$. The experiences and results gained from these studies will help researchers to obtain higher quality and more reliable data using spectroscopic methods in living animals. Not every preclinical research institute has the access or knowledge to acquire spectra in living animals. The original spectra released might be beneficial for them. The dataset can also be made part of a larger database with spectra for data mining to a comprehensive scientific output. Another potential use of the dataset is as a machine learning training dataset or other in other advanced data processing approaches to optimize further background subtraction modules, which might lead to a more universal and standardized data processing method of Raman spectra.

In current clinical practice, the strong excitation light needed in the Raman spectroscopy damages the fragile posterior section of the eye. Hence, we designed darkfield probe designs, which no direct illumination light reaching the retina. They are discussed in Chapters 5 and 6. Using these to obtain in-vivo ocular drug concentrations in 
the animal eye will be of great help in evaluating the efficiency drug delivery into the anterior eye chamber when administrated topically. This might even be improved using methods like AADF presented in Chapter 7. The experimental settings and parameters proposed here may help physicists to establish such spectroscopic measurement systems for use in pre-clinical applications.

\section{Social impact}

Raman spectroscopy and mass spectroscopy are both very specific diagnostic techniques that provide the results in short time. That's why the Dutch minister of Public Health, Hugo de Jonge, recommended them as candidates for testing Covid-19 patients. However, only Raman spectroscopy is suitable for medical in vivo diagnostic applications. As the Raman effect is a very weak phenomenon, it is difficult to apply this technique directly in the vulnerable living eye.

Therefore, a safe way for utilizing Raman spectroscopy in ophthalmology will benefit a wider audience outside the scientific community in various forms. For clinicians and ophthalmologists, real-time molecular profiling will accelerate research procedures in clinical practices. For patients the burdens of biopsies will be significantly reduced by using non-invasive, in situ approaches like Raman spectroscopy. Animals might not necessarily be sacrificed to acquire drug data if these non-invasive molecular assessment methods become available. Hence, the usage of lab animals can be reduced. The economic benefit of reducing the number of lab animals will drive the pharmaceutical companies to broaden the usage of the Raman technique as an innovation in the industry. Furthermore, the granted patent might inspire and spread the application of the Raman technique in the medical device startups and companies. The biomarker measurement techniques by Raman spectroscopy and dark-field probes proposed in this thesis will be available and promoted to both scientific and industrial communities as commercial products and services. 
Page | iv 


\section{Appendix II}

Patent

Shuo Zhang

Page | V 
(12) INTERNATIONAL APPLICATION PUBLISHED UNDER THE PATENT COOPERATION TREATY (PCT)

(19) World Intellectual Property Organization

International Bureau

(43) International Publication Date 11 May 2018 (11.05.2018)

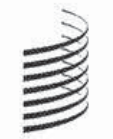

W $!$ P O । PCT

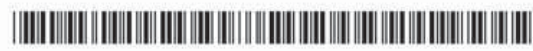

(10) International Publication Number

WO 2018/083112 Al
(51) International Patent Classification: A61B 3/10 (2006.01) A61B 5/00 (2006.01)

A61B 5/1455 (2006.01)

(21) International Application Number:

PCT/EP20 17/077952

(22) International Filing Date:

01 November 2017 (01.11.2017)

(25) Filing Language:

English

(26) Publication Language:

English

(30) Priority Data: 16196751.8

01 November $2016(01.11 .2016)$ EP

(71) Applicants: UNIVERSITEIT MAASTRICHT [NL/NL]; Minderbroedersberg 4-6, 6211 LK MAASTRICHT
(NL). ACADEMISCH ZIEKENHUIS MAASTRICHT [NL/NL]; P. Debyelaan 25, 6229 HX MAASTRICHT (NL).

(72) Inventors: ZHANG, Shuo; c/o Universiteit Maastricht, Minderbroedersberg 4-6, 6211 LK MAASTRICHT (NL). BERENDSCHOT, Toussaint Theresia Johannes Maria; c/o Universiteit Maastricht, Minderbroedersberg 4-6, 6211 LK MAASTRICHT (NL). ERCKENS, Roel Johan; c/ o Universiteit Maastricht, Minderbroedersberg 4-6, 6211 LK MAASTRICHT (NL). JONGSMA, Franciscus, Hermanus, Maria; Dwarsstraat 2, 6235 BX Ulestraten (NL). DE BRABANDER, John; Doctor Larijweg 39, 7961 NM Ruinerwold (NL).

(74) Agent: ALGEMEEN OCTROOI- EN MERKENBUREAU B.V.; P.O. Box 645, 5600 AP EINDHOVEN (NL).

(81) Designated States (unless otherwise indicated, for every kind of national protection available): $\mathrm{AE}, \mathrm{AG}, \mathrm{AL}, \mathrm{AM}$,

(54) Title: DEVICE FOR PERFORMING MEASUREMENTS OF THE CHEMICAL COMPOSITION OF THE ANTERIOR EYE AS WELL AS AN INTEGRATED OPTICAL UNIT FOR IMPLEMENTATION THEREIN

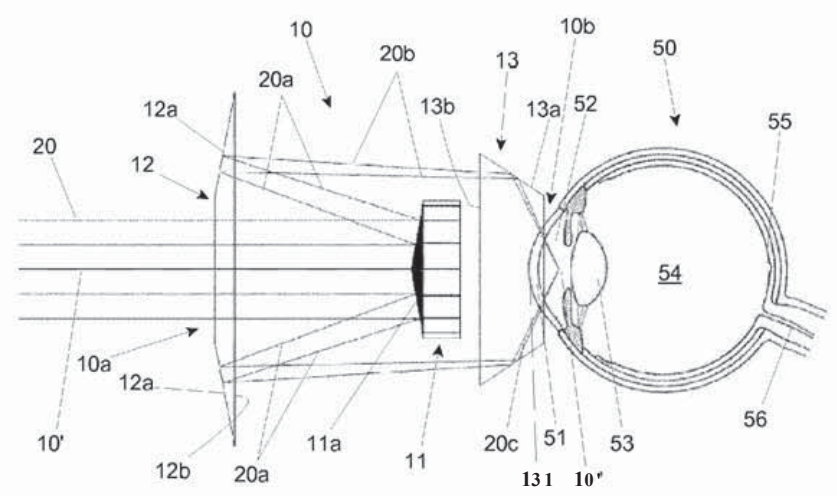

Fig. 2

(57) Abstract: The invention relates to device for determining the chemical composition of a living eye comprising light emitting means N for emitting a light beam; light guidance means having an entrance surface and an exit surface and arranged to be brought in optical - contact with the eye for directing said light beam being emitted towards the eye, such that at least part of the eye is being illuminated with a light beam having an oblique angle of incidence with respect to the visual axis of the eye and for receiving and guiding at least $\infty$ a fraction of the light beam leaving the eye as a result of said illumination towards light detecting means for determining the chemical (C) composition of said eye by analyzing said fraction of light. The invention also relates to an optical unit for implementation in such a $\mathbf{0 0}$ device for performing measurements of the chemical composition of the anterior eye.

0 


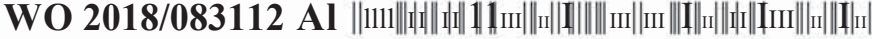

$\mathrm{AO}, \mathrm{AT}, \mathrm{AU}, \mathrm{AZ}, \mathrm{BA}, \mathrm{BB}, \mathrm{BG}, \mathrm{BH}, \mathrm{BN}, \mathrm{BR}, \mathrm{BW}, \mathrm{BY}, \mathrm{BZ}$ $\mathrm{CA}, \mathrm{CH}, \mathrm{CL}, \mathrm{CN}, \mathrm{CO}, \mathrm{CR}, \mathrm{CU}, \mathrm{CZ}, \mathrm{DE}, \mathrm{DJ}, \mathrm{DK}, \mathrm{DM}, \mathrm{DO}$, DZ, EC, EE, EG, ES, FI, GB, GD, GE, GH, GM, GT, HN, HR, HU, ID, IL, IN, IR, IS, JO, JP, KE, KG, KH, KN, KP, $\mathrm{KR}, \mathrm{KW}, \mathrm{KZ}, \mathrm{LA}, \mathrm{LC}, \mathrm{LK}, \mathrm{LR}, \mathrm{LS}, \mathrm{LU}, \mathrm{LY}, \mathrm{MA}, \mathrm{MD}, \mathrm{ME}$, MG, MK, MN, MW, MX, MY, MZ, NA, NG, NI, NO, NZ, OM, PA, PE, PG, PH, PL, PT, QA, RO, RS, RU, RW, SA, SC, SD, SE, SG, SK, SL, SM, ST, SV, SY, TH, TJ, TM, TN, TR, TT, TZ, UA, UG, US, UZ, VC, VN, ZA, ZM, ZW

(84) Designated States (unless otherwise indicated, for every kind of regional protection available): ARIPO (BW, GH, GM, KE, LR, LS, MW, MZ, NA, RW, SD, SL, ST, SZ, TZ, UG, ZM, ZW), Eurasian (AM, AZ, BY, KG, KZ, RU, TJ, TM), European (AL, AT, BE, BG, $\mathrm{CH}, \mathrm{CY}, \mathrm{CZ}, \mathrm{DE}, \mathrm{DK}$, EE, ES, FI, FR, GB, GR, HR, HU, IE, IS, IT, LT, LU, LV MC, MK, MT, NL, NO, PL, PT, RO, RS, SE, SI, SK, SM, TR), OAPI (BF, BJ, CF, CG, CI, CM, GA, GN, GQ, GW, KM, ML，MR, NE，SN, TD, TG)

Published:

- with international search report (Art. 21(3)) 
Device for performing measurements of the chemical composition of the anterior eye as well as an integrated optical unit for implementation therein.

\section{FIELD OF THE INVENTION}

The invention relates to device for determining the chemical composition of a living eye comprising light emitting means for emitting a light beam; light guidance means having an entrance surface and an exit surface and arranged to be brought in optical contact with the eye for directing said light beam being emitted towards the eye, such that at least part of the eye is being illuminated with a light beam having an oblique angle of incidence with respect to the visual axis of the eye and for receiving and guiding at least a fraction of the light beam leaving the eye as a result of said illumination towards light detecting means for determining the chemical composition of said eye by analyzing said fraction of light.

The invention also relates to an integrated optical unit for implementation in such a device for performing measurements of the chemical composition of the anterior eye.

\section{BACKGROUND OF THE INVENTION}

Many industrial, scientific and medical processes involve the measurement of the chemical composition of tissue of the human body for a variety of applications. In most cases the accuracy of the measurements is of great importance for the quality of the output of the process mentioned. A specific type of tissue measurements involve the measurement of the transparent media of the eye, as is applied, for example, in ophthalmology where proteins, pharmacological substances, or other molecules in aqueous humor are determined, wherein the presence of these substances provide an indication of the quality of the eye and sometimes information concerning underlying diseases.

Most methods of measuring the pathologic conditions in the eye are based on chemical and histological techniques to acquire information on molecule changes in the tissue or identification of pathogens which implies the use of spectroscopic techniques like absorption, scattering, electronic spin, and/or mass spectroscopy.

The limitations of these methods are that the most of them are performed in vitro, which makes invasive procedures in the eye necessary that may led to complications like hypotony or an endophthalmitus of the eye and is a frightening 
intervention from the patients point of view.

A solution is the application of in vivo spectroscopy, especially with Raman spectroscopy. Raman spectroscopy offers an opportunity to detect molecules qualitative as well quantitative and is a valuable tool to investigate biological materials in aqueous environments. For Raman spectroscopy in the living eye a light beam is focused in the tissue of interest and the back scattered light comprising the Raman signals is gathered, e.g. in a confocal device, and analyzed in a spectrometer.

International patent application no. WO2004/098397 discloses such an in vivo application of Raman spectroscopy, wherein an oblique illumination with respect to the visual axis of the eye of the anterior chamber of the eye is effectuated in such a way that the incident light beam is not aimed at the retinal tissue. In WO2004/098397 a focusing lens with a curvature conformal to the wave front of the light beam leaving the lens is implemented with the aim of avoiding directing or aiming light at fragile, retinal tissue, and thus limiting radiation damage.

However such optical solution suffers from the drawback of chromatic aberration. The focusing lens causes different wavelengths of light beam to have different focal lengths at or in the part of the eye to be illuminated, resulting in a distorted back scattered light beam being reflected back from said illuminated part of the eye. Likewise said distorted reflected light beam contains insufficient and incorrect Raman signals, which do not provide qualitative as well quantitative information as to the presence and composition of biological materials in the aqueous environment of the anterior chamber of the eye.

\section{SUMMARY OF THE INVENTION}

The present invention aims to provide a solution to the above problems, and to provide a more accurate device for the noninvasive measurement of qualitative as well quantitative information as to the presence and composition of biological materials in the aqueous environment of the anterior chamber of the eye.

In addition it is an object of the present invention to provide a device for performing more accurate measurements of the chemical composition of tissues or scattering properties with a real time integration length control, for monitoring changes in ocular tissue or in situ biomaterials, and for monitoring ocular pharmacokinetics.

Hereto in the device according to the invention, seen in the direction of propagation of said light beam being emitted from the light emitting means through the 
light guidance means towards the eye, said light guidance means comprises a system of at least a first mirror element, a second mirror element and a third mirror element, at least one of said three mirror elements having a non-planar mirror surface.

With this optical solution chromatic aberration is absent when directing the light beam towards the part of the eye to be illuminated. In particular the backscattered light beam is not distorted and the Raman signals contained therein provide far more accurate (in terms of quality as well as quantity) information as to the presence and composition of biological materials in the aqueous environment of the anterior chamber of the eye.

More in particular in an embodiment the first mirror element, seen in the direction of propagation of said light beam being emitted from the light emitting means through the light guidance means towards the eye, is the mirror element having a nonplanar mirror surface. As such a proper reflection of the light beam entering the light guidance means is obtained towards the part of the eye to be illuminated wherein a maximum part of the entering light beam reaches said part of the eye. This results in a significant backscatter part of light being reflected back from the eye with Raman signals thus provide more (in terms of quality as well as quantity) information for analysis and diagnosis.

Said first mirror element having a non-planar mirror surface can be a convex mirror element or a spherical convex mirror element.

In yet another implementation said convex mirror is an aspherical convex mirror, which may exhibit a sag $z$ following the expression:

$$
z=\frac{c r^{2}}{1+\sqrt{1-(1+k) c^{2} r^{2}}}+\beta_{1} r^{1}+\beta_{2} r^{2}+\cdots
$$

Herein is $c$ is the curvature of the mirror surface, $r$ is the radial coordinate (in $\mathrm{mm}$ ), $\mathrm{k}$ is the conic constant, and in an embodiment $\mathrm{k}=-1, \mathrm{c}=1$, and $\beta \mathrm{r}$ is in the range between $-0,1$ and $-0,3$ and in particular $\beta 1=-0,2$ and $\beta 2$ is in the range between $3,5 \times 10^{-3}$ and $4,5 \times 10^{-3}$, in particular in the range between $3,75^{*} 10^{-3}$ and $4,25 \times 10^{-3}$ and more in particular $\beta_{2}=4,169151^{*} 10^{-3}$.

In another embodiment of the device the second mirror element, seen in the direction of propagation of said light beam being emitted from the light emitting means through the light guidance means towards the eye, is the mirror element having a nonplanar mirror surface. Also with this embodiment similar improved results are obtained with regard to the maximum part of the light beam illuminating the part of the eye to be 
examined, and a significant backscatter part of light being reflected back from the eye with Raman signals thus providing more (in terms of quality as well as quantity) information for analysis and diagnosis.

In this embodiment said second mirror element having a non-planar mirror

5 surface is a concave mirror in particular an elliptical concave mirror exhibiting a sag $z$ following the expressions:

$$
z=\frac{c r^{2}}{1+\sqrt{1-(1+k) c^{2} r^{2}}}
$$

and

$$
k=-\frac{\left(\mathbf{a}^{2}-b^{2}\right)}{a^{2}}
$$

Here $c$ is the curvature of the mirror surface, $r$ is the radial coordinate (in $\mathrm{mm}$ ), $\mathrm{k}$ is the conic constant, $\mathrm{a}$ is the semi major axis of the elliptical mirror surface (in $\mathrm{mm}$ ), and $b$ is the semi minor axis of the elliptical mirror surface (in $\mathrm{mm}$ ). In an embodiment $a$ is in the range between 10 and $20 \mathrm{~mm}$, in particular in the range between 12.5 and $17.5 \mathrm{~mm}$, and more in particular $a=15 \mathrm{~mm}$, and wherein $b$ is in the range between 15 and $25 \mathrm{~mm}$, in particular in the range between 17.5 and $22.5 \mathrm{~mm}$, and more in particular $b=20,137427 \mathrm{~mm}$.

Furthermore the other mirror elements of said system of at least three mirror elements are planar mirrors. Thus the light beam which enters the light guidance means is only reflected via a system of mirror elements prior to entering and illuminating a part (the anterior chamber) of the eye. No distortion as with a system of lenses takes place, and from the resulting backscatter light beam with Raman signals more (in terms of quality as well as quantity) information for analysis and diagnosis can be obtained.

In particular said planar third mirror element is positioned directly near the eye for reflecting said light beam having an oblique angle of incidence with respect to the visual axis of the eye.

Moreover the system of at least three mirror elements is rotational symmetric with respect to the visual axis of the eye simplifying the handling and operation of the device by a physician when performing Rama spectroscopy on an eye.

Furthermore the light guidance means may comprise a collimating lens positioned before the first mirror element, seen in the direction of propagation of said light beam being emitted from the light emitting means through the light guidance means towards the eye. 
In addition said light guidance means consisting of said system of at least the first mirror element, the second mirror element and the third mirror element and optionally said collimating lens are constructed as an integrated optical unit. This also improves handling and operation of the device.

In a further improvement said light emitting means comprise a laser device, resulting in an parallel light beam when entering the light guidance means.

The invention also relates to an integrated optical unit for implementation in a device for performing measurements of the chemical composition of the anterior eye, wherein said integrated optical unit is functioning as light guidance means comprising an assembly of at least a first mirror element, a second mirror element and a third mirror element according to the invention.

\section{BRIEF DESCRIPTION OF THE FIGURES}

The invention will now be described in more detail with reference to the accompanying drawings, which drawings show in:

Figure 1 a schematic view of an embodiment of a device according to the invention;

Figure 2 an embodiment of light guidance means for implementation in a device for performing measurements of the chemical composition of the anterior eye according to the invention;

Figures $3 a-3 c$ side views from the embodiment of Figure 2;

Figures 4 another embodiment of light guidance means for implementation in a device for performing measurements of the chemical composition of the anterior eye according to the invention;

Figures $5 a-5 c$ side views from the embodiment of Figure 3

Figures $6 \mathrm{a}-6 \mathrm{~g}$ detailed views of an embodiment of the invention constituted as an integrated optical unit consisting of an assembly of mirror elements

Figure 7 a schematic view of another embodiment of a device according to the invention. 


\section{DETAILED DESCRIPTION OF THE FIGURES}

For a better understanding of the invention like parts in the drawings are denoted with like reference numerals.

The present invention has three functions: focusing of a beam of excitation light in the eye in such a way that no direct light hits the retinal tissue, to preserve the optical quality of the excitation beam as is provided by the collection lens, and to collect, with a high aperture, the in the eye scattered light.

A first embodiment of a device in which the present invention can be used is shown in Figure 1 in a schematic manner and not to scale.

The device denoted with numeral 1 of Figure 1 has light emitting means 5 , e.g. a laser device, that emits an collimated and coherent laser beam 20. The laser or light beam 20 has a constant or nearly constant diameter with a minimal divergence as it propagates through the device along it's optical path. Light beam 20 passes a dichroic beam-splitting mirror 7 and enters light guidance means 10 .

Light guidance means 10 are depicted in Figure 1 in a schematic manner as a box, but are composed of a system of at least three mirror element $s$, as it will be explained in more detail with reference to the Figures 2-5. Preferably the light guidance means 10 are manufactured as an integrated optical unit with the three mirrors incorporated and assembled together.

Coherent laser or light beam 20 enters the light guidance means 10 via an entrance face $10 \mathrm{a}$, propagates through the system of at least three mirror element $s$ and leaves the light guidance means 10 via the exit face $10 \mathrm{~b}$ before it enters and illuminates part of the eye 50 of a human or animal being for diagnosis purposes.

Hereto the exit face $10 \mathrm{~b}$ of the light guidance means 10 is formed/shaped as to be placed against the eye ball with a minimum of discomfort, and usually a film of contact fluid is present between the exit face 10 and the eye 50 . The light guidance means 10 and in particular the system of at least three mirrors divert the light beam 20 from the visual axis 10' (see Figure 2) such that an oblique illumination with respect to the visual axis of the eye 50 of the anterior chamber of the eye 50 is effectuated in such a way that the incident light beam 20 is not aimed at the retinal tissue.

Within the anterior chamber (the area of interest) of the eye 50 the impinging light beam 20 is back scattered, said back scattered light comprising the Raman signals. Said Raman signals are representative to the qualitative as well quantitative information pertaining to the presence and composition of biological materials 
in the aqueous environment of the anterior chamber of the eye 50 .

Said back scattered light will follow the same optical path through the light guidance means 10 in the direction of the dichroic beam-splitting mirror 7, where the back scattered light, now denoted with reference numeral $20^{\prime}$ is collected and reflected to light

5 detection means 6 , where it is analyzed. Said light detecting means 6 may comprise a spectrometer with a sensor, such as a charge coupled device (CCD) or such as a photo multiplier.

Preferably the light detection means 6 are not part of the light emitting means 5 . However in another embodiment (not shown) both the light detection means 6 and the light emitting means 5 can be incorporated in one construction device, thereby obviating the mirror 7 .

Figure 2 and Figure $3 a-3 b-3 c$ disclose a first embodiment of the light guidance means 10 according to the invention. The light guidance means 10 are constructed preferably as an integrated optical unit which is rotational symmetric around an axis, in the Figures represented as and coinciding with the visual axis 10' of the eye 50 .

As indicated previously in the introduction of the description, the invention relates to Raman spectroscopy wherein light is directed into a part of the eye, in particular the anterior chamber 52. In particular light is entering the anterior chamber 52 of the eye

2050 at an oblique angle of incidence with respect of the axis 10' of the eye 50 . Herewith it is avoided that light impinging the eye illuminates fragile tissue such as the eye lens 53 , the vitreous humour 54 , the retina 55 or the optical nerve 56 .

In particular the integrated optical unit forming the light guidance means 10 exhibit an entrance face $10 \mathrm{a}$ and an exit face 10b/131. In particular the exit face 10b/131 25 is formed or shaped conformal to the curvature of the eye ball 51 of the eye 50 allowing a proper optical contact with the eye and to minimize any discomfort for the human or animal being under examination.

The light guidance means 10 comprise an assembly or a system of at least three mirror elements denoted with reference numerals 11,12 and 13 . The three mirror elements 11,12 and 13 are accommodated in and more in particular assembled together as the integrated optical unit 10. The first mirror element 11, the second mirror element 12, and the third mirror element 13 are positioned such that the light beam 20 follows a propagation path, such that it is reflected in a sequential order via the first, second and third mirror elements $11,12,13$ before it enters the part of the eye 50 to be illuminated, in 
particular the anterior chamber 52 .

According to the invention at least one mirror of said system of three mirror elements $11,12,13$ exhibits a non-planar reflection surface. In the embodiment as shown in Figure 1 it is the first mirror element 11 seen in the direction of propagation of the light 5 beam 20 that exhibits said non-planar reflection surface 11a. The other two mirror elements $12-13$ of said optical system of mirrors have a planar reflection surface denoted with reference numerals $12 a$ and $13 a$ respectively.

A collimated and coherent laser beam 20 is directed parallel to the optical axis $10^{\prime}$ of the eye 50 (which also corresponds with the rotational symmetric axis of the light guidance means 10) and impinges on the non-planar reflection surface 11a of the first mirror 11. Light beams 20a are reflected from said first mirror 11 and impinge on the planar mirror surface $12 \mathrm{a}$ of the second mirror 12 . Light beams $20 \mathrm{~b}$ being reflected from the planar mirror surface 12a of the second mirror 12 enters the (forward) entrance surface $13 \mathrm{~b}$ of the third mirror 13 and are subsequently reflected at the planar mirror surface 13a at the back side of the third mirror 13 (seen in the direction of propagation of the light beams $20 \mathrm{~b}$ ).

Light beams 20c being reflected from the planar mirror surface 13a of the third mirror element 13 enter at an oblique angle of incidence relative to the optical axis 10 ' of the eye 50 the anterior chamber 52 . Said reflected light beams $20 \mathrm{c}$ converge in the anterior chamber 52 near point 10" where a part of said incident light beams $20 \mathrm{c}$ is backscattered. Said backscattered part of the incident light beam contains Raman signals essential for performing Raman spectroscopy and is reflected back following the same optical path of propagation as the light beam 20 entering the light guidance means 20 via the entrance surface 10a. Backscattered light containing the Raman signals is reflected back via the third mirror element 13 , the second mirror element 12 and the first mirror element 11 towards light detection means 6 as depicted in Figure 1.

The non-planar mirror surface $11 a$ of the first mirror element 11 is preferably a convex mirror or a spherical convex mirror. However, preferably said convex mirror exhibits an aspherical convex mirror surface which may exhibit a sag $z$ following the expression:

$$
z=\frac{c r^{2}}{1+\sqrt{1-(1+k) c^{2} r^{2}}}+\beta_{1} r^{1}+\beta_{2} r^{2}+\cdots
$$

Herein is $c$ is the curvature of the non-planar mirror surface $11 a, r$ is the radial coordinate relative to the optical axis $10^{\prime}, \mathrm{k}$ is the conic constant. In a particular 
embodiment of the non-planar mirror surface $11 a k=-1, c=1$ and $\beta_{1}$ is in the range between 0 and -1 , in particular between $-0,1$ and $-0,3$ and more in particular $\beta_{1}=-0,2$ and $\beta 2$ is in the range between 0 and 0,1 , in particular between $3,5 \times 10^{-3}$ and $4,5 \times 10^{-3}$, more in particular in the range between $3,75^{*} 10^{-3}$ and $4,25 \times 10^{-3}$ and furthermore in particular $\beta 2$ $5=4,169151 \times 10^{-3}$.

By implementing a light guidance means 10 composed of a system of only mirrors, the light beam 20 entering the light guiding means 10 via the entrance surface $10 \mathrm{a}$ is only reflected. As such, a maximum part of the light beam entering the light guidance means 10 can reach the part of the eye of interest, in particular the anterior chamber of the eye. Hence, with only a system of mirrors, a larger amount of light entering the anterior chamber of the eye will generate a more significant backscatter part of light, that is being reflected back from the eye with Raman signals towards the light detection means 6 (see Figure 1). As such, this integrated optical unit provides more information for analysis and diagnosis of the presence and composition of biological materials in the aqueous environment of the anterior chamber of the eye, both in terms of quality as well as in quantity.

Unlike Raman spectroscopy applications, which implement lenses for directing and focusing light towards to the anterior chamber of the eye, with the present invention the optical drawbacks of chromatic aberration are avoided and more accurate non-invasive measurements concerning the presence and composition of biological materials in the aqueous environment of the anterior chamber of the eye are obtained.

As it will be shown in the different views of Figures $2 a-2 b-3 c$ it is clear that the second mirror 12 forms an open annulus through which the light beam 20 can enter via entrance face 10a in the direction of the reflective mirror surface 11a of the first mirror 11. Seeing along the optical axis $10^{\prime}$ the first mirror 11 is positioned between the second mirror 12 which is orientated parallel with the entrance surface 10a of the light guidance means 10 , and the third mirror 13 which forms the exit surface 10b/131 of the light guidance means 10 .

In the embodiment of Figure 4 and Figures $5 a-5 b-5 c$ another embodiment of the light guidance means is shown, wherein the light guidance means are depicted with reference numeral 100. Also in this embodiment the light guidance means 100 can be constructed as an integrated optical unit which is rotational symmetric around the optical axis $100^{\prime}$.

Also in this embodiment light propagates or follows the same optical path 
through the light guidance means 100 as in the embodiment of the light guidance means 10 of Figures 2 and 3 . However, in the second embodiment the first mirror 11 and the third mirror 13 are planar mirrors, whereas the second mirror 12 has a non-planar mirror surface $12 \mathrm{a}$. concave mirror surface and more in particular an elliptical concave mirror exhibiting a sag $z$ following the expressions:

$$
z=\frac{c r^{2}}{\mathbf{1}+\sqrt{1-(1+k) c^{2} r^{2}}}
$$

and

$$
k=\frac{\left(a^{2}-b^{2}\right)}{a^{2}}
$$

Here $c$ is the curvature of the non-planar mirror surface $12 a, r$ is the radial coordinate (in $\mathrm{mm}$ ) relative from the optical axis 100', $\mathrm{k}$ is the conic constant, a is the semi major axis (in $\mathrm{mm}$ ) of the elliptical mirror surface 12a, and $b$ is the semi minor axis (in $\mathrm{mm}$ ) of the elliptical mirror surface 12a. In an embodiment $\mathrm{a}$ is in the range between 10 and $20 \mathrm{~mm}$, in particular in the range between 12.5 and $17.5 \mathrm{~mm}$, and more in particular a $=15 \mathrm{~mm}$, and $\mathrm{b}$ is in the range between 15 and $25 \mathrm{~mm}$, in particular in the range between 17.5 and $22.5 \mathrm{~mm}$, and more in particular $\mathrm{b}=20,137427 \mathrm{~mm}$.

Also with this embodiment a maximum part of light is only reflected through the optical guidance means 100 towards the anterior chamber of the eye and no distortion as with a system of lenses will take place. A maximum part of light entering the eye will be backscattered with Raman signals containing more in terms of quality as well as quantity information for analysis and diagnosis.

The different views of Figures $6 \mathrm{a}-6 \mathrm{~g}$ depict in more detail an embodiment of the optical unit 10 as previously depicted and described in Figure 2 and 3 in its disassembled state (figures 6a-6b) as well as in its assembled state (Figure 6c-6g). The second mirror 12 is shaped as a hollow cylindrical optical element, having an open annulus 10a which acts as entrance face for the light beam 20 in the direction of the reflective mirror surface 11 a of the first mirror 11 . The hollow cylindrically shaped second mirror 12 exhibit an open inner space 120, which exposes the reflective mirror surface $11 \mathrm{a}$ of the first mirror 11 to the impinging light beams 20a.

The first mirror 11 is shaped as a disc-shaped element, which fits accurately in a circular recess 132 present in the optical element 13 , which functions as 
the third mirror. The disc-shaped first mirror 11 and the recess 132 are preferably circular, due to the rotational symmetric design of the integrated optical unit 10 around the axis $10^{\prime}$ (see Figure 2). However also other shapes, such as a square shape are possible depending on the design of the optical unit 10 .

First mirror element 11 preferably exhibits an outer dimension which is slightly larger than the inner dimensions of the recess 132, allowing the first mirror 11 being press fit into the recess 132 of the third mirror element 13 without using additional adhesives, such as glue, which might adversely affect the optical transmission of the light beams 20 through the integrated optical unit 10 .

Second mirror element 12 and third mirror element 13 are assembled together, preferably also with the means of a press fit connection, thereby obviating adhesives. However as the contact surfaces of the second and third mirror elements 1213 are positioned outside the propagation path of the impinging light beams $20 \mathrm{a}-20 \mathrm{~b}-20 \mathrm{c}$ as well as the reflected Raman signals back towards the light detecting means 6 (see

15 Figure 1) with the first mirror element 11 accommodated in the recess 132 of the third mirror element 13, an adhesive can be used, as no disturbance of the impinging light beams and returning Raman signals will occur.

The light beams 20 enter the optical unit 10 via entrance face or opening $10 \mathrm{a}$, propagate through the open inner space 120 of the hollow cylindrically shaped second mirror element 12 and impinge on the non-planar reflection surface 11a of the first mirror 11. Light beams $20 \mathrm{a}$ are reflected from said non-planar reflection surface $11 \mathrm{a}$ and impinge on the planar mirror surface $12 \mathrm{a}$ of the second mirror 12 . Light beams $20 \mathrm{~b}$ being reflected from the planar mirror surface $12 \mathrm{a}$ of the second mirror 12 enters the (forward) entrance surface $13 \mathrm{~b}$ of the third mirror element 13 and are subsequently reflected at the planar mirror surface $13 a$ at the back side of the third mirror 13 (seen in the direction of propagation of the light beams 20b).

Light beams $20 \mathrm{c}$ being reflected from the planar mirror surface $13 \mathrm{a}$ of the third mirror 13 enter at an oblique angle of incidence the anterior chamber of the eye. The exit surface $10 \mathrm{~b}$ of the third mirror 13 is formed as an circular dent having an inner surface dimension or surface contour 131 , which is conformal to the curvature of the eye ball 51 of the eye 50 allowing a proper optical contact with the eye and to minimize any discomfort for the human or animal being under examination (not shown in Figures 6a-6g, but see Figure 2).

In Figure 7 another embodiment is disclosed, where said light guidance 
means 10 (or integrated optical unit 10) further comprises a collimating lens 15 positioned before the first mirror element 11 , seen in the direction of propagation of said light beam being emitted. The device 5 exhibits a laser device 7 , that emits a non-collimated laser beam 2. The laser or light beam 2 enters the collimating lens element 15 resulting in 5 collimated and coherent laser beam light 20 having a constant or nearly constant diameter with a minimal divergence as it propagates through the integrated optical unit 10 along it's optical path towards the first mirror element 11 .

The collimating lens element 15 can be integrated with the integrated optical unit 10, where lens element 15 is mounted/connected against the entrance face 10a of the second mirror element 12 .

The device according to the invention can also be used for measuring biomaterials in the anterior eye or for monitoring changes in the chemical composition of tissues or biomaterials. The device according to the invention is also suitable for monitoring ocular pharmacokinetics. 


\section{CLAIMS}

1. Device for determining the chemical composition of a living eye comprising: light emitting means for emitting a light beam; light guidance means having an entrance surface and an exit surface and arranged to be brought in optical contact with the eye for directing said light beam being emitted towards the eye, such that at least part of the eye is being illuminated with a light beam having an oblique angle of incidence with respect to the visual axis of the eye and for receiving and guiding at least a fraction of the light beam leaving the eye as a result of said illumination towards

light detecting means for determining the chemical composition of said eye by analyzing said fraction of light,

wherein, seen in the direction of propagation of said light beam being emitted from the light emitting means through the light guidance means towards the eye,

15 said light guidance means comprises a system of at least a first mirror element, a second mirror element and a third mirror element, at least one of said three mirror elements having a non-planar mirror surface.

2. Device according to claim 1, wherein, seen in the direction of propagation of said light beam being emitted from the light emitting means through the light guidance

20 means towards the eye, the first mirror element is the mirror element having a non-planar mirror surface.

3. Device according to claim 2, wherein said first mirror element having a nonplanar mirror surface is a convex mirror element in particular a spherical convex mirror element or an aspherical convex mirror element.

25 4. Device according to claim 3, wherein said aspherical convex mirror element exhibits a sag $z$ following the expression:

$$
z=\frac{c r^{2}}{1+\sqrt{1-(1+k) c^{2} r^{2}}}+\beta_{1} r^{1}+\beta_{2} r^{2}+\cdots
$$

with: $\quad c$ is the curvature of the mirror surface

$$
r \text { is the radial coordinate (in } \mathrm{mm} \text { ) }
$$

$\mathrm{k}$ is the conic constant.

5. Device according to claim 4 , wherein

$\mathrm{k}=-1, \mathrm{c}=1$ and

$\beta \mathrm{r}$ is in the range between 0 and -1 , in particular between $-0,1$ and $-0,3$ and 
more in particular $\beta_{\imath}=-0,2$ and

$\beta 2$ is in the range between 0 and 0,1 , in particular between $3,5 \times 10^{-3}$ and $4,5 \times 10^{-3}$, more in particular in the range between $3,75^{*} 10^{-3}$ and $4,25 \times 10^{-3}$ and further more in particular $\beta_{2}=4,169151^{*} 10^{-3}$.

56 .

Device according to claim 4 or 5 , wherein

$\mathrm{k}=-1, \mathrm{c}=1$ and

$\beta_{1}$ is in the range between $-0,1$ and $-0,3$ and in particular $\beta_{1}=-0,2$ and

$\beta 2$ is in the range between $3,5 \times 10^{-3}$ and $4,5^{*} 10^{-3}$, in particular in the range between $3,75 \times 10^{-3}$ and $4,25^{*} 10^{-3}$ and more in particular $\beta_{2}=4,169151 \times 10^{-3}$.

107.

Device according to claim 1 , wherein, seen in the direction of propagation of said light beam being emitted from the light emitting means through the light guidance means towards the eye, the second mirror element is the mirror having a non-planar mirror surface.

8.

Device according to claim 7 , wherein said second mirror element having a non-planar mirror surface is a concave mirror, in particular an elliptical concave mirror.

9. Device according to claim 8, wherein said elliptical concave mirror exhibits a sag $z$ following the expressions:

$$
z=\frac{c r^{2}}{1+\sqrt{1-(1+k) c^{2} r^{2}}}
$$

and

with:

$$
k=\frac{\left(a^{2}-b^{2}\right)}{a^{2}}
$$

$\mathrm{C}$ is the curvature of the mirror surface

$r$ is the radial coordinate (in $\mathrm{mm}$ )

$\mathrm{k}$ is the conic constant

$a$ is the semi major axis of the elliptical mirror surface (in $\mathrm{mm}$ )

25 $\mathrm{b}$ is the semi minor axis of the elliptical mirror surface (in $\mathrm{mm}$ ).

10.

Device according to claim 9 , wherein

$a$ is in the range between 10 and $20 \mathrm{~mm}$, in particular in the range between 12.5 and $17.5 \mathrm{~mm}$, and more in particular $\mathrm{a}=15 \mathrm{~mm}$, and wherein

$b$ is in the range between 15 and $25 \mathrm{~mm}$, in particular in the range between 17.5 and $22.5 \mathrm{~mm}$, and more in particular $b=20,137427 \mathrm{~mm}$.

11. Device according to any one of the preceding claims, wherein the other mirror elements of said system of at least three mirror elements are planar mirrors. 
12. Device according to claim 11, wherein said planar third mirror is positioned directly near the eye for reflecting said light beam having an oblique angle of incidence with respect to the visual axis of the eye.

13. Device according to any one of the preceding claims, wherein the system

5 of at least three mirrors is rotational symmetric with respect to the visual axis of the eye. 14. Device according to any one of the preceding claims, wherein said light guidance means further comprises a collimating lens positioned before the first mirror element, seen in the direction of propagation of said light beam being emitted from the light emitting means through the light guidance means towards the eye.

Device according to any one of the preceding claims, wherein said light guidance means consisting of said system of at least the first mirror element, the second mirror element and the third mirror element and optionally said collimating lens element are constructed as an integrated optical unit.

16. Integrated optical unit for implementation in a device for performing

15 measurements of the chemical composition of the anterior eye, said integrated optical unit functioning as light guidance means comprising an assembly of at least a first mirror element, a second mirror element and a third mirror element according to one or more of the preceding claims. 


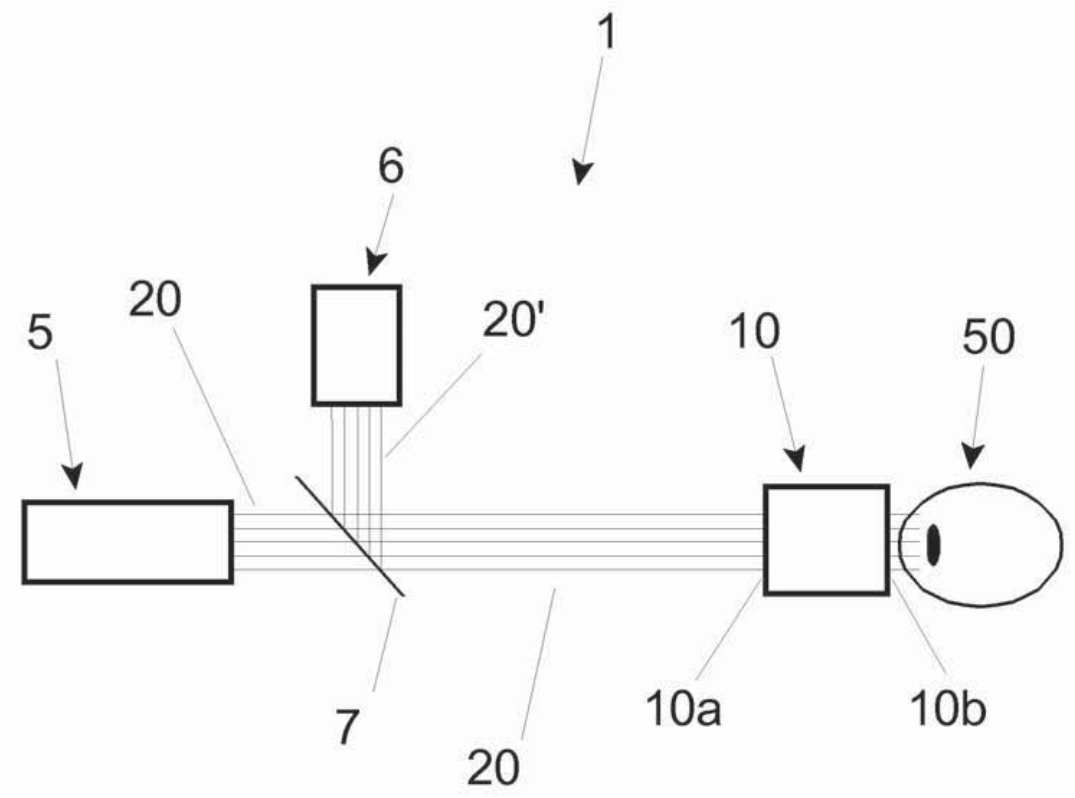

Fig. 1 


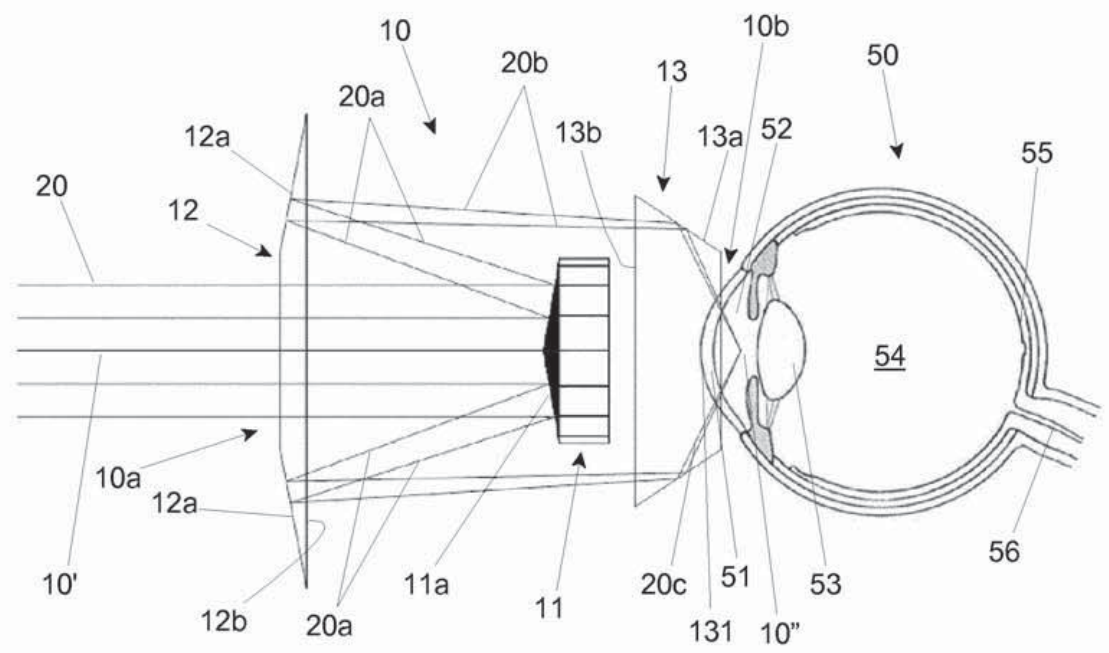

Fig. 2 


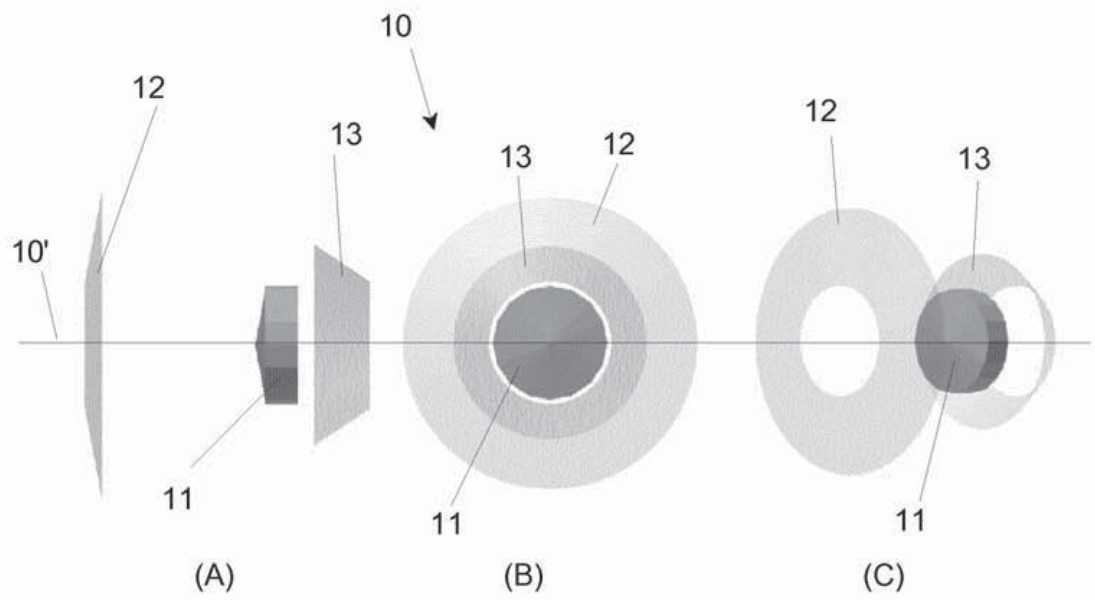

Fig. 3 


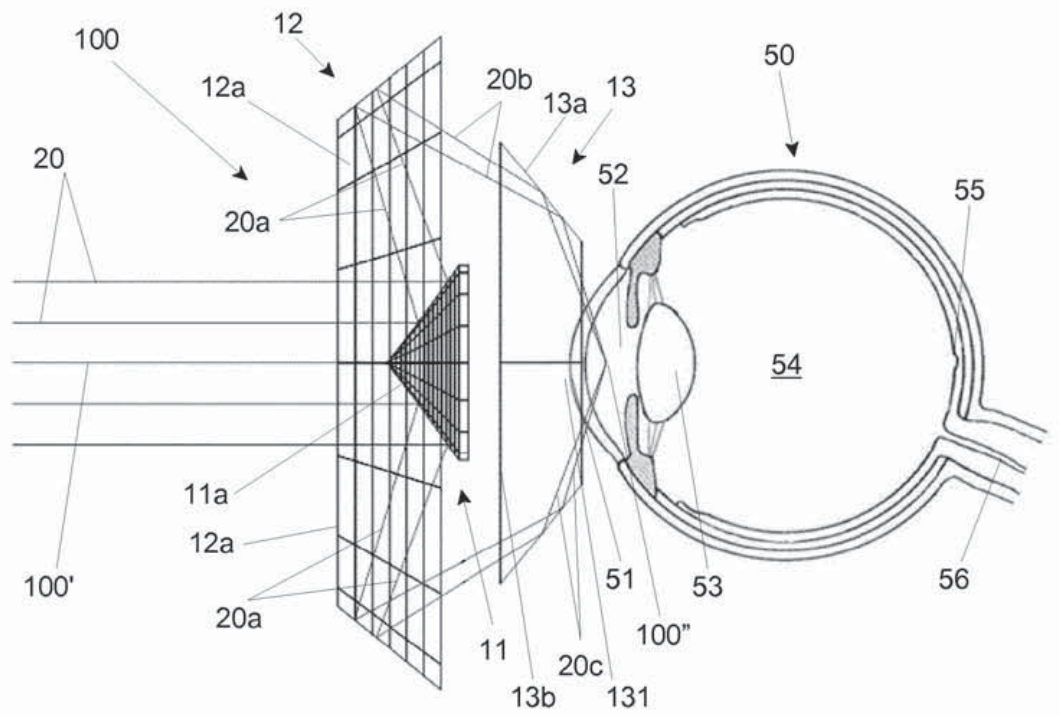

Fig. 4 


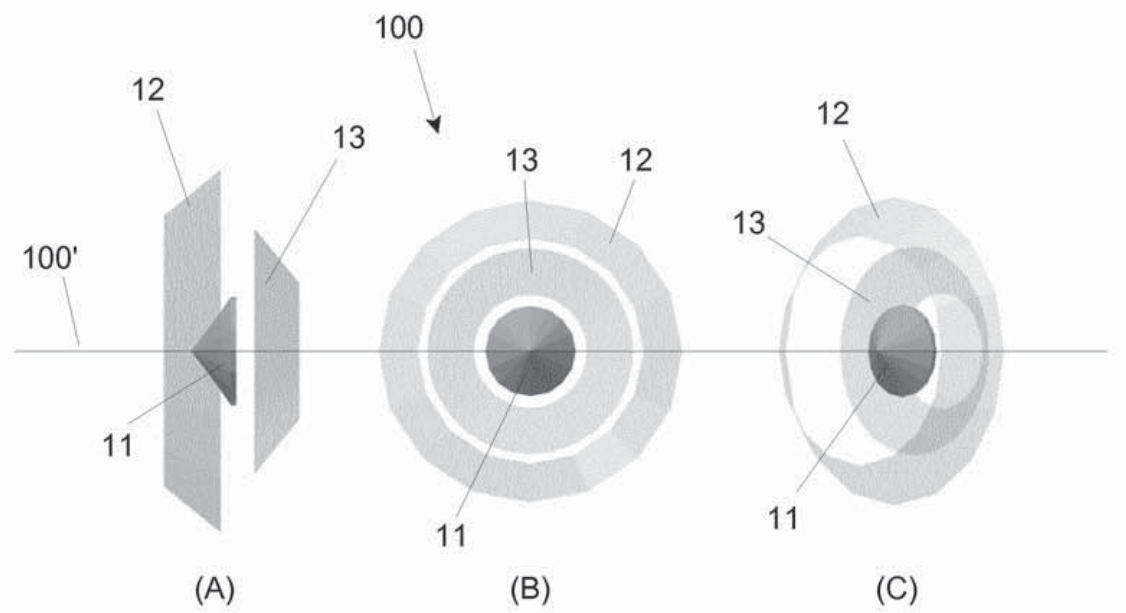

Fig. 5 


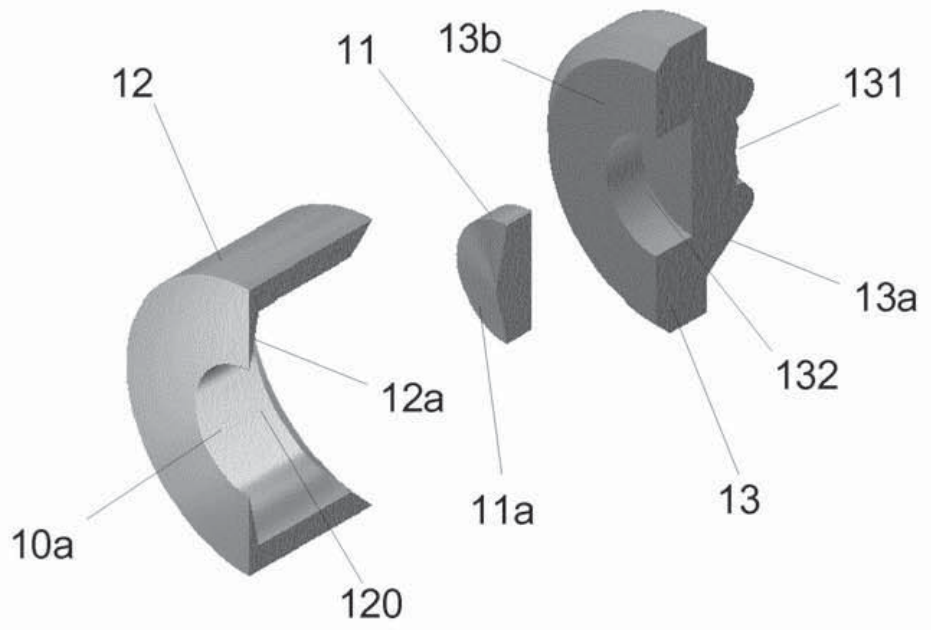

(A)

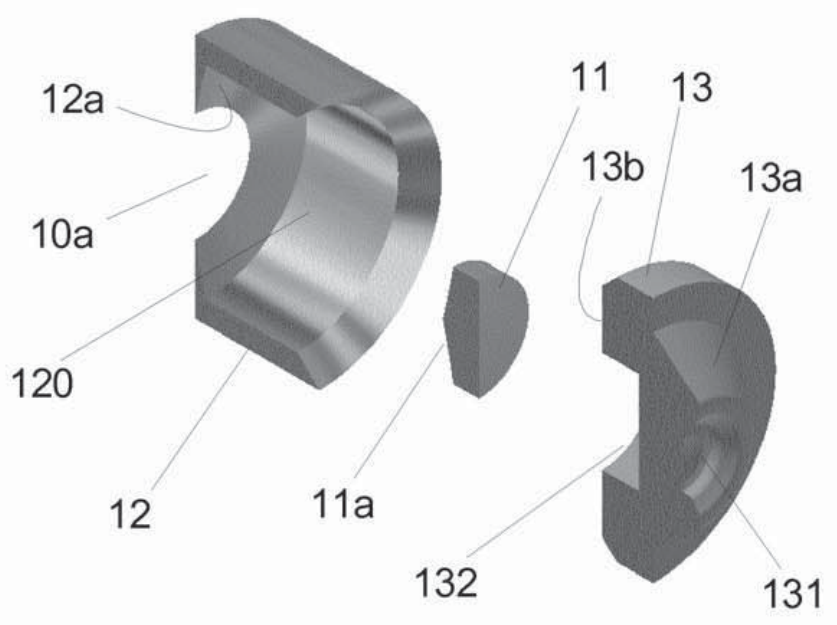

(B)

Fig. 6 

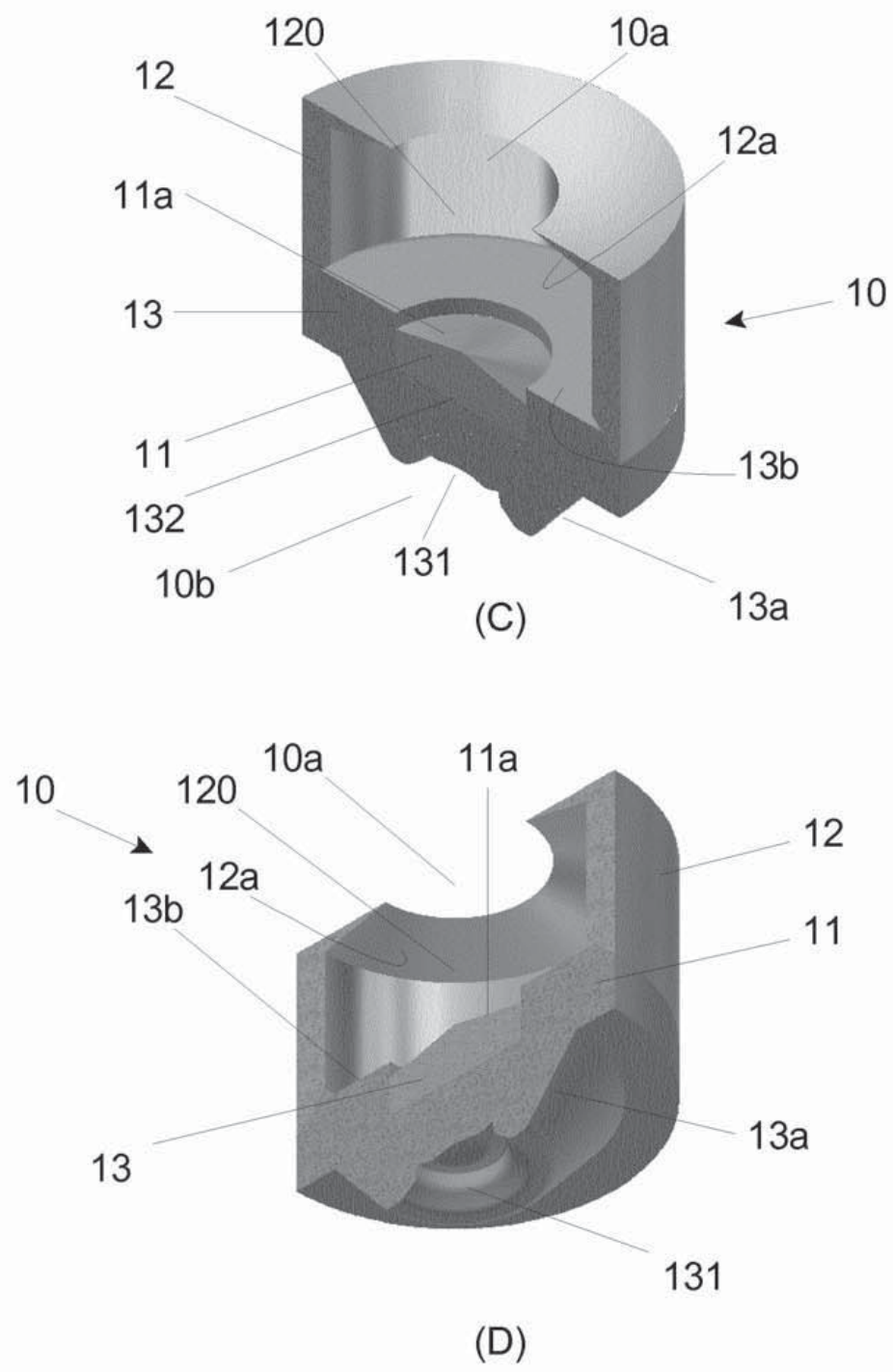

Fig. 6 


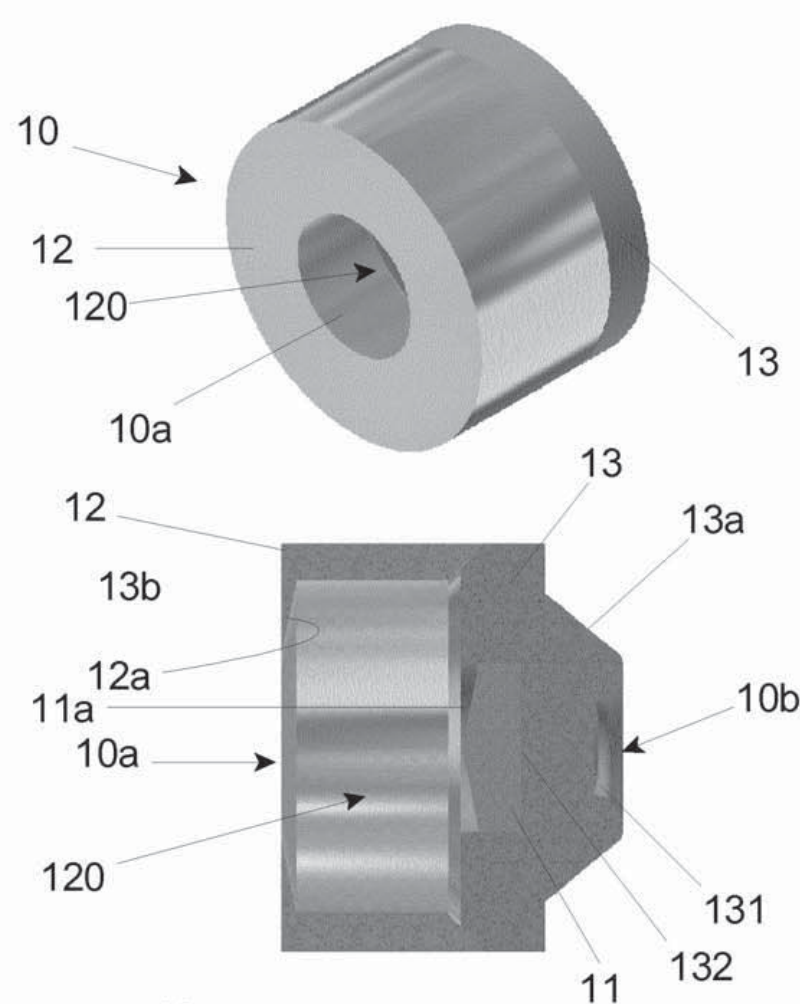

(E)

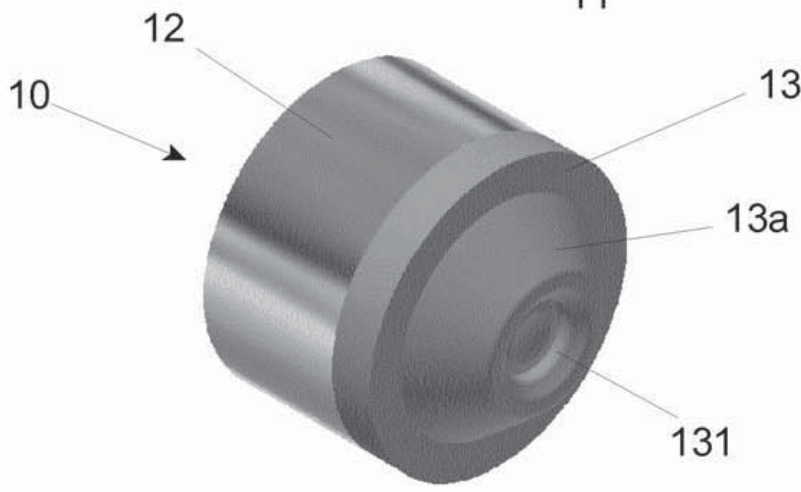

(F)

(G)

Fig. 6 


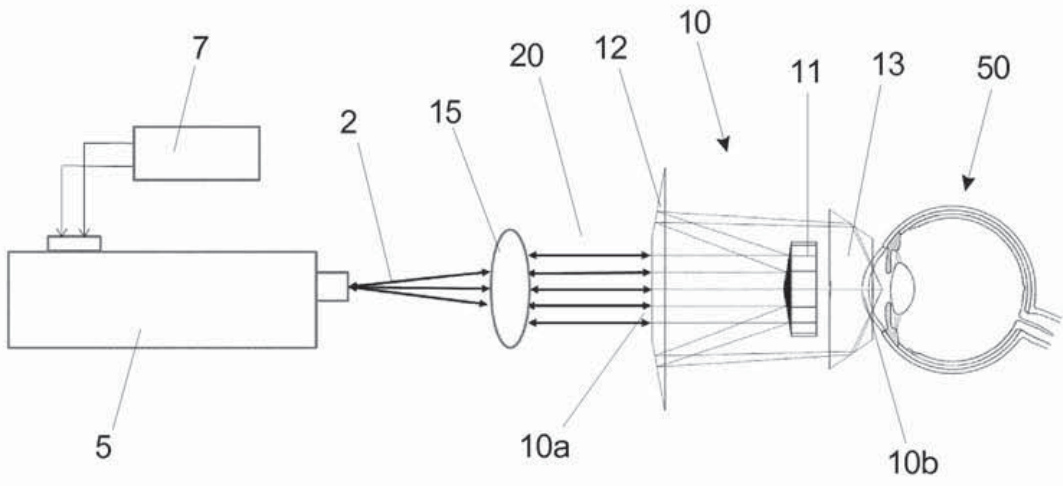

Fig. 7 
INTERNATIONAL SEARCH REPORT

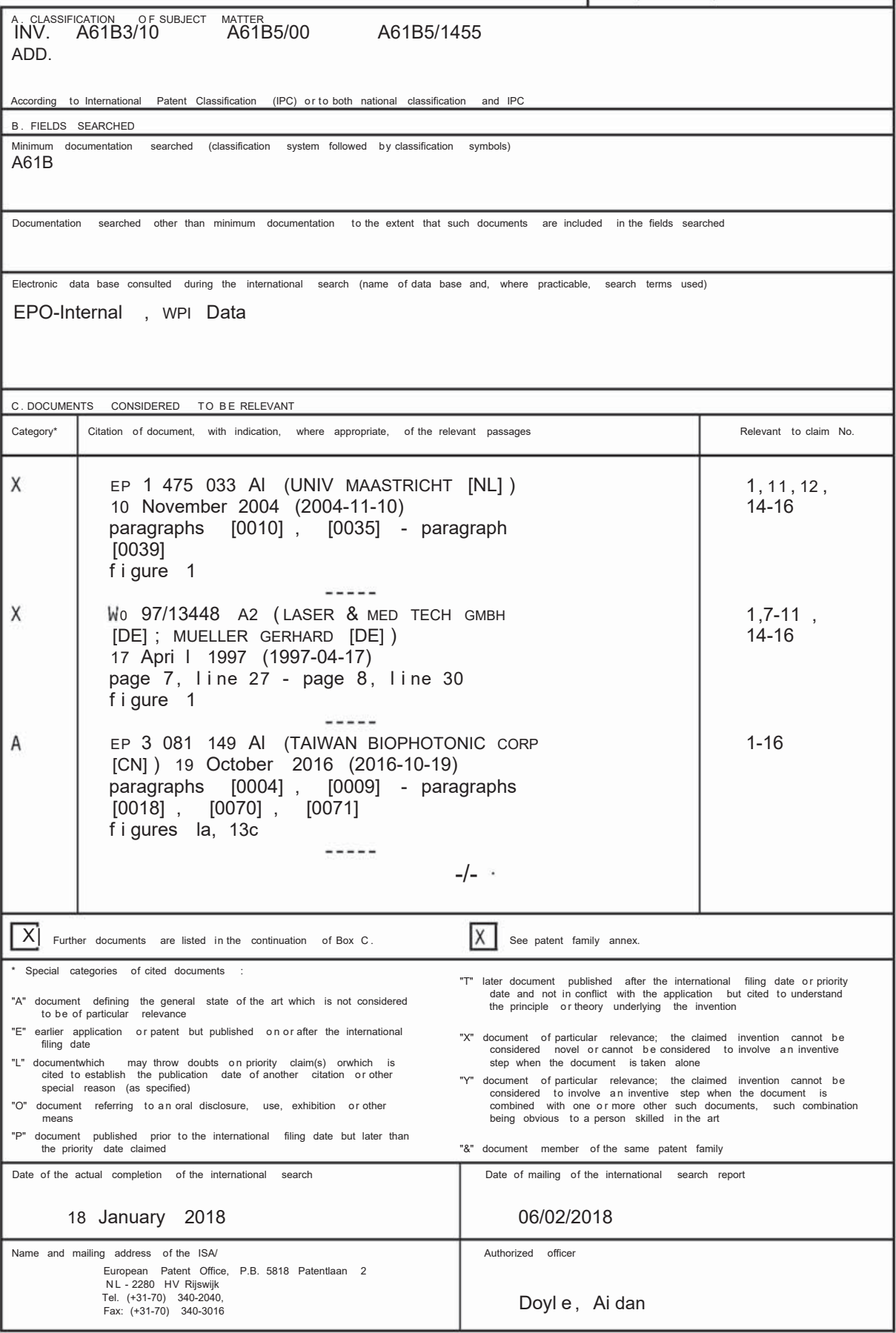

Form PCT/ISA/210 (second sheet) (April 2005) 


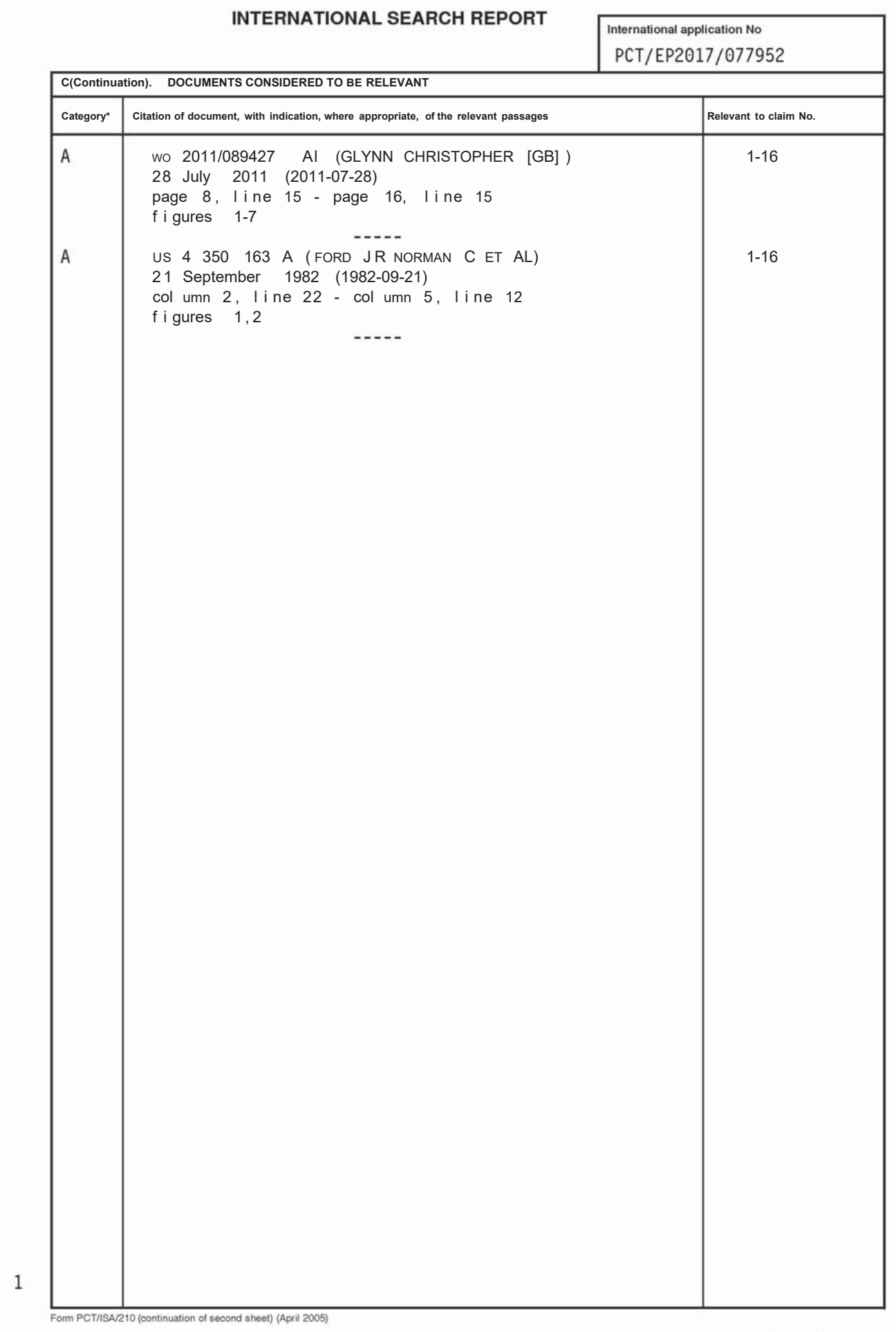




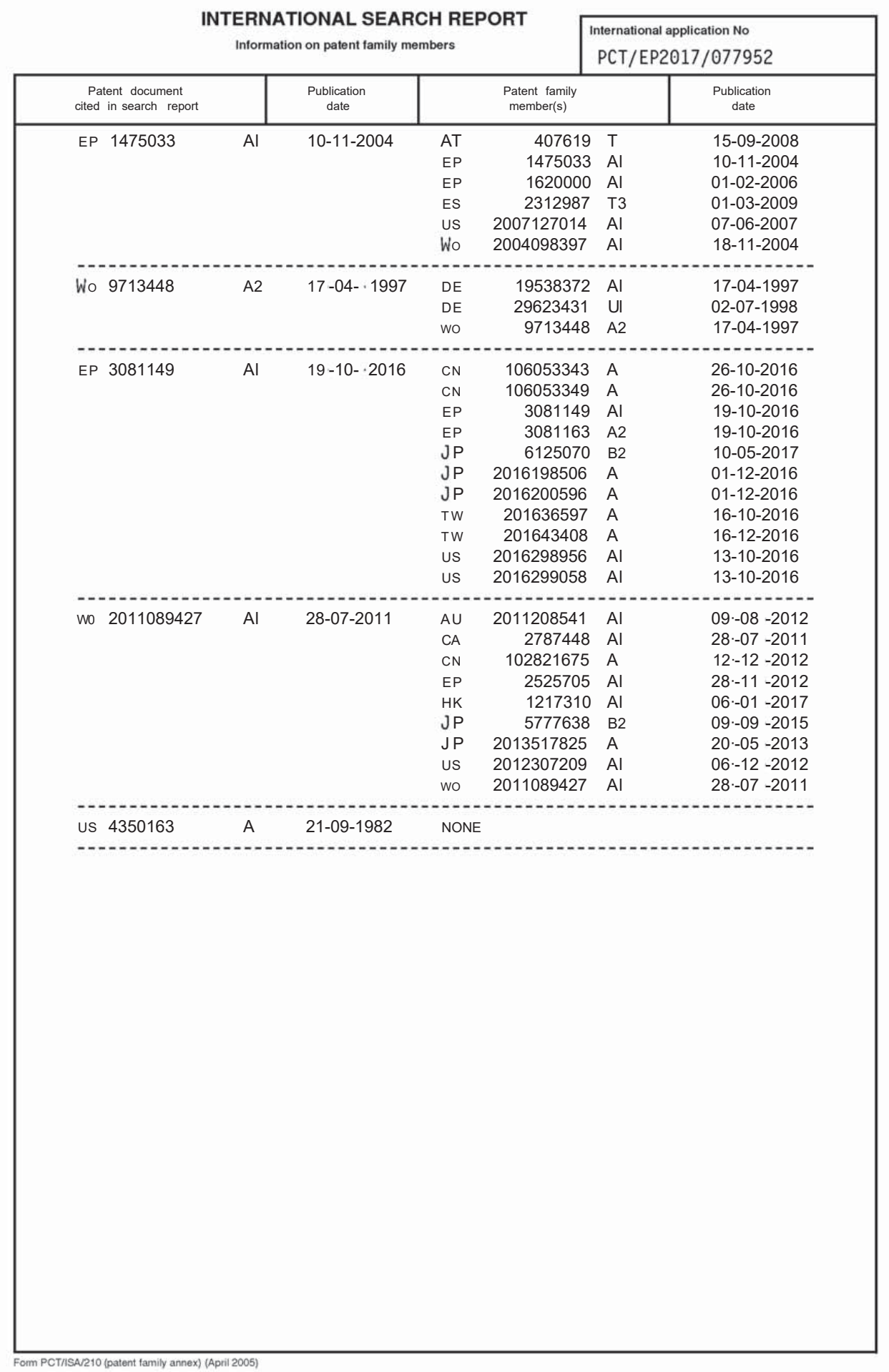




\section{Appendix III}

Acknowledgements/致谢

Shuo Zhang

Page | xxxv 
First, I would like to acknowledge the Chinese Scholarship Council (CSC) for the funding that covers my living cost in the Netherlands. I also would like to acknowledge Maastricht University and University Eye Clinic Maastricht for all the support provided during my $\mathrm{PhD}$ research.

I would like to express my sincere gratitude to the members of my $\mathrm{PhD}$ thesis assessment committee: Prof. J. Prickaerts (Chair), Prof. M.A.M.J. van Zandvoort, Prof. O. Bekers, Prof. J.H. de Boer and Dr. G.J. Puppels. Thank you for your valuable feedbacks and comments.

The support from my promotion team is precious. Prof. Carroll Webers, Dr. Tos Berendschot and Dr. Roel Erckens, I couldn't achieve all these scientific outputs and made the progress of the research in the journey towards my $\mathrm{PhD}$ degree. I learned how to do science in a rigorous way. The promotion team showed me how to digest a research question and guide me walking through it steps by steps until it becomes a scientific output. Prof. Carroll Webers, your guidance always from a bigger picture and directly to the point, I could have a chance to observe closely what is a real prestigious scientist and distinguished ophthalmologist. The time is always short and never enough to learn and study beside you. Dr. Tos Berendschot, thank you for contacting me and offering such great research opportunities to join this team and doing research that I could really use my expertise and fulfill my dream. Thank you for teaching me these new techniques, your attention to the details made me realize how sloppy I am before I enroll as a $\mathrm{PhD}$ student here. The PhD training I had here is mostly from you and you are so smart in problemsolving and excellent in almost all technical related works. I am deeply appreciated the support from you. Dr. Roel Erckens, through your eye I could realize what is research really needed for the clinical practice. Your insights are so priceless and unreplaceable. Sometimes the questions you asked are hard to answer. However, these are the times that I realized to improve myself in the way of science, these are the steps you made for me to step closer into the scientific truth, these are the ladders to higher achievements. Besides, I also enjoyed the spare time with you, you provided the opportunities to experience the things that I can barely do by myself, like sailing and another hobby of you (that I will keep it off-record :-D). You are living a life that I dreamed of with these "toys" you have, like telescope, motorcycles, boat, guns and even play station, etc., it is man's dream and romance. I would like to express my sincere gratitude for all the things you have done for me both in the research and spare time. All in all, I am so lucky and proud to be the student of this fantastic supervisory team and I think I must have saved the earth in my past life so that I could have the opportunity to study under your supervision.

Dr. Frans Jongsma and Dr. Johny de Brabander, thank you for your guidance for the optic design, your ideas and many years' experience (your experience in optic might even longer than my age) in optic plays a key role in my research. Thank you for your patience when I asked the optic questions, I really admire your expertise and passion for optics and ophthalmology. You are so knowledgeable about this field and you both will be 
the role models of me that urge me to learn in a lifelong time. You are the perfect example of an old Chinese saying, never too old to learn. Theo, I heard so many compliments of you from everyone I know and I know they are not all of it. Thank you for all the beneficial discussions and suggestions. Hope there are possibilities to collaborate with you, it must be a fantastic experience and can learn a lot from you.

Prof. Harry Steinbusch, thank you for introducing me to the local community and helping me familiar with the university system. You are so nice and care about me, whenever I met problems, you are always willing to give me a hand and help me to solve them. Thank you for inviting me to have the dinner in your place to show how locals live and what it looks like inside a Dutch house when I just arrived and not have a close friend yet. I sincerely dreamed about I could earn those achievements like you and gain your wisdom at your age. Thank you again, Sir Harry Steinbusch, the honor is mine to know you in my life.

Christian my dearest friend, I still remember the first time we met. It was on a team building day while visiting a farming tools exhibition, you and Wouter just entering it right after me. This is where all the stories begin, for all those activities that did together like climbing, kayaking, hiking, BBQ, tasting Chinese food, camping and traveling, etc., those good memories will be the lifetime treasures of mine. No matter where I am if you need me I will be there for you, I wish you all the best for the future not only in your career but also with Aline.

Sylvia, I am very lucky and glad to know you in the Maastricht. Your achievement in the work and talent in the violin impressed me so much. I am also very enjoyed the relaxed time with you, so peaceful. Looking forward to hearing from you with any progress you made and any new hiking route you found.

Suryan, it is always nice chatting with you. I remember the first time meet you is on the second floor of the eye tower when I was waiting for Dr. Roel Erckens, I should say you are looking good in a white coat as a doctor. I didn't expect that we have so many in common for the hobbies, your climbing skills are professional level and the best climber I ever know, if I need a coach it definitely you. I am also surprised that you have been to China for one year, it is my pleasure to host you with all the Chinese delicious food in the way you like. You are also good at photography, the way you look at the world is unique. Life is about the choice you make, I am not supervised whatever you decided to do in the future, it must be the best choice and decision.

Luigi, thank you for being one of the paranymphs of me, the endless help you offered means a lot to me. You explained how things going in the Netherlands, translated what people saying when they have conversations in Dutch. Your professional in the field, warm hearted to the people and the vision of the future grantee a bright future that beyond I could possibly imagine.

Melinda, it is my pleasure to be the office roommate with you. You offered help when I was hesitating about the career choice, your experience and insight are so valuable for me 
both in academia and industry. All the effort paid off, I am so glad that you found the position that suits you the best.

Claudette, you always prepared even for playing Mahjong, a traditional Chinese board game. I am so impressed that you even did some homework and printed the rules for it before joining. I couldn't see anything that could stop you when pursuing your goals. All difficulties or obstacles are temporary, you will conquer them sooner or later, I have faith in you without any doubt.

Wouter, I would like to call you the king of the video games and hardcore fan of play station (or toasty? haha). Through you, I know what ACG looks like in Europe and really envy you about the chance to join Gamescom (one of the biggest game event in the world). I also like your research topic and the amount of time you invested in the lab work is much more than it looks like. I really enjoyed those moments when you were explaining your works next to your poster in our office.

Jackson, hey mate, thank you for your help in correcting my English from the perspective of a native speaker. It is really fun to having drinking moments with you, always something new to hear and discuss. I also learned so many ways to say hi and goodbye, you never repeated yourself, so creative and energetic. (I know this paragraph probably still needs some corrections or improvements, looking forward to your feedbacks. :-D) As for Chris, Lunch?

It is my honor to work with so many passionate and talented colleagues, I received so much supports, kindly greetings, interesting discussions from each of you, Pascal, Lindsay, Ralph, Lotte, Ilona, Jarno, Magali, Maartje, Ellis, Soraya, Jurriaan, Marlies, Frank, Thomas, Antoni and many, many friends might forget to list here, your effective working habit, good personalities and responsibility definitely deserve all the success.

To my dear Chinese friends, without your support I also could not achieve this. 依稀 记得来马城前很幸运的先认识了李婕一同学, 如果没有你和赵俊芳恐怕我们在马城 就要露宿街头了, 谢谢你们夫妇让我在你们家借宿了两周直到我们租到了的房子。 谢谢赵俊芳不厌其烦的指导我们从史基浦机场下飞机到马城的全程事无巨细的指导 和工作时间还抽空到火车站接我们, 让我们消除了初到异乡的陌生和不安。在荷兰 的这些年一起旅行, 一起入坑摄影, 一起工作和学习都是美好的回忆, 作为 “别人 家的孩子”，祝你和婕一在新的工作岗位上前程似锦，万事胜意。

认识王利同学是在一次看球的喝酒活动中, 干脆利落爱憎分明（还好支持的是 一个球队, 哈哈) 的性格给我留下了深刻的印象, 因为对于运动的共同爱好而相谈 甚欢, 自此就和于亮组成了铁三角, 一起看球, 一起聚餐, 一起钓鱼, 一起旅行, 一起运动。谢谢你能在我找不到人的时候一起在冬天报名参加网球课, 风里来雨里 去不间断, 让我能在运动中有一个好对手好伙伴。于亮于老板也功不可没, 划船, 击剑样样不缺, 一起跑步时展现出与体型不相称的速度让自训经常运动的我自叹弗 如。铁三角聚餐时的普法讲座也着实让我涨了很多的知识, 让我领略了学科交叉的 
魅力。听闻你和王利自回国后各自取得不菲的成绩, 愿小伙伴们鹏程万里, 生活锦 绣。

谢谢宗胜华同学分享的人生感悟, 因为处在相似的人生阶段, 有着相近的个人 和家庭状况, 所以能更多更深入的交流这个阶段独有的苦与乐, 关于科研, 关于职 业规划, 关于家庭, 关于责任和选择, 很感激能遇到同路人一起趟过坑迈过坎。和 徐青交流不多, 但每次都有帮助都有收获。希望你们夫妇前路无碍, 皆是通途。

王蜀金同学的勇气, 执著和果敢给与了我很多的力量, 你一旦决定便毫无顾虑 勇往直前的作风一直是我向往和学习的榜样。也许荷兰求学之路没有如预期版顺利, 但你以力破巧硬是趟出了一条自己的路, 取得了令人敬佩的成绩。勇往直前的风格 也延续到了篮球上, 一身腱子肉也是我努力的方向, 以后一定要再找机会一起打球, 实现当时一起参加全荷赛时未竟的梦想。想你以后不管是生活还是工作一定会重剑 无锋, 锋芒毕露。

罗倩, 感谢疫情期间组织的各种户外羽毛球篮球足球局, 能在附近和小伙伴一 起运动的感觉简直不要太好, 连加班的时候都更有动力了呢, 哈哈。也许未来还未 来, 不管今后阴晴如何变幻, 相信你也会扶摇直上, 鹏程万里。

金涵让我认识到了在游戏, 旅行, 摄影上什么叫人外有人, 很享受和你聊起这 些话题时内容涉及的深度和广度, 交流时你对专业, 社会和未来那些清晰深刻的远 见也让我受益匪浅。你和全乐的每次招待都非常贴心, 得知你们未来的规划很为你 们开心, 前方等待你们的一定是星辰大海, 睘宇九州。

虽然和罗鸿幸相熟未久, 但可以说是相见恨晚, 每次聊起来都有说不完的话。 谢谢你让我领略和学习到了什么叫企业家精神, 你对本领域精深的理解和一线实操 细致入微的洞察让我折服, 我毫不怀疑你实现理想的能力和魄力, 而永婵总能在我 们讨论陷入苦恼时给出灵光一闪的崭新思路。商场如战场, 我在你身上既看到了诸 葛亮般运筹帷幄的智慧也看到了赵云般万军从中取敌首级的勇武, 希望今后能以我 微薄之力助你未来披荆斩棘, 所向披靡。

荣世松, 我依旧清晰地记得我们相遇的那一天, 17 年 5 月 6 号, 在 ARVO imaging meetings 上。当时我在看一个吸烟相关数据分析的展板（展厅入门第六个）, 讲解人对我的问题不太了解, 我很庆幸听从了他的建议等你来解释。当时只道是寻 常, 但是现在回头看, 感觉我的未来从此有了一条全新的路。我第一次对志同道合 这个词有了直观和切身的体会, 知道了有个可靠强大的伙伴一起去实现梦想是什么 感觉。从此不管是春节还是圣诞, 我们每周一次的会都未曾间断, 每次都在向目标 一点一点迈进的踏实感让我远离了任何焦虑, 因为我知道我们走在正确的道路上, 也终将会到达。任何表达感谢的言语都是不足的, 唯有加倍努力, 不负期待。对于 你的未来不敢妄加揣测, 但我无比肯定它一定超越任何人的想象, 没有极限。 
赵沅蒂, 都都, 岳洋, 梦莹, 很高兴可以一起喝酒, 一起 $\mathrm{BBQ}$, 一起八卦, 也 算是疫情前最轻松的回忆了, 没心没肺的吃喝玩乐的日子总是很短暂, 还想一起喝 酒吹水看深夜的马城, 让同学年少, 青春不老。

当过邻居的陈兾, 还有一起打球一起运动的小伙伴艺文, 大庆, 老顾, 杨桂林, 曾海燕, 希望大家的身体都越来越好, 兄弟赵宇希望你在国内一切都好, 事业重要, 身体一样重要, 期待下次见到一个红光满面的你, 不要再每次都很憔悴的样子。

张书赫感谢你让我重温手撕公式的美好, 让我还记得自己是个货真价实的理科 生, 我有预感以后一起还会发文章而且还不会少, 有机会一定要再一起搞事情, 哈 哈。还有太多的小伙伴在日常的学习和生活中留下了深刻的印象, 乔奇我一定会把 我们的文章发一个好杂志, 你也一定会在新的岗位上做出成绩来, 坚持就是胜利;

Sunny 姐, 每次和您交流都会对荷兰的社会, 商业, 校企合作, 成果转化有进一步 的了解, 谢谢您带我认识了荷兰学校之外的一面, 谢谢您带来的商务活动的机会, 让我获益匪浅。萍姐, 张明, 辛莹, 陈琳, 傲敏, 油文婷, 路畅, 贾雨霏, 安宁, 杨荣辉, 李雯婕, 蔡路, 朱晓庆, 刘轶林, 胡伟凡, 邓奇林, 薛艳红老师, 饶圣样, 王简琴, 荼英等等各位小伙伴, 感谢一路以来的支持和帮助, 不管大家今后是否在 一个地方, 是否经常联系, 但是一起学习和生活的经历给予了我面对遇到困难时无 论何时何地都能汲取的源源不断的力量。

爸爸, 妈妈, 安攀还有晞景, 谢谢你们的支持以及原谅我的任性, 让我不管什 么年纪都依旧可以继续追寻自己的梦想。没有父母的教诲与言传身教, 我不会是现 在的我, 爸爸给予了我最宝贵的好奇心, 让我可以对这个世界充满求知欲, 也可以 对抗实验室无数个看上去很枯燥的日子; 妈妈展现的责任感与对患者的大爱也是我 现在和未来选择的原动力, 让我想用自己的所学所会去切实的帮助更多的人, 去解 决他们遇到的问题, 去减轻他们可能受到的痛苦, 去让他们一样可以享受生活和世 界的美好。安同学使我成为了一个更为完整的人, 补完了人字的另外一笔。你的包 容和支持自始至终没有变过, 过往的一切依旧鲜活, 一起看过日出日落, 见过高山 大海, 去过古刹教堂, 探过古迹深林, 游过中西内外。感谢你的陪伴, 今后不管是 崎岖还是坦途, 余生皆是前路, 我们一起走。晞景, 谢谢你的出现, 让我有了新的 身份与责任, 有了憧憬和希望, 有了动力和能量走下去, 而你也会有自己的路, 勇 敢去追寻不要畏惧。 


\section{Appendix IV}

Curriculum Vitae

Shuo Zhang

Page | xli 
Page | xlii 
Shuo Zhang was born on $18^{\text {th }}$ January 1985 in Hebei province, China. When he was a child, he was deeply impressed by the beauty of physics through the eyes of his father who is an electronics engineer. Then he started his career to be a physicist, obtained his Bachelor degree from Hebei University in major of Optical information science and technology in 2008, follow obtained his Master degree from North-West Normal University in the major of Optics in 2011. He worked on devising a portable mass spectrometer at the Chinese Academy of Science thereafter from 2011 to 2013. Later on, He was inspired by his mother's work as a nurse, by whom he was deeply touched by the conditions that patients suffering. He decided to apply his knowledge in physics for translational biomedical optics applications. Therefore, he embarked on his $\mathrm{PhD}$ project of transforming Raman spectroscopy into a clinical diagnostic device at MUMC and Maastricht university, the Netherlands. 
Page | xliv 


\section{Appendix V}

List of Publications

Shuo Zhang 


\section{Manuscripts}

1. S. Zhang*, F.A.M. van der Mee, R.J. Erckens, C.A.B. Webers, T.T.J.M. Berendschot. Raman Spectroscopy detection of Interleukin-10 and Angiotensin Converting Enzyme. Journal of the European Optical Society - Rapid publications, 2021,17 (7)

2. S. Zhang, R.J. Erckens, F. Jongsma, C.A.B. Webers, T.T.J.M. Berendschot*. Design and performance of a dark field probe with confocal-Raman spectroscopy for ophthalmic applications. Journal of Raman spectroscopy, 2021,1 (5).

3. S. Zhang", SH Zhang", T.T.J.M. Berendschot*. Harnessing abruptly auto-defocusing beam to enhance the Raman signal in aqueous humor: a simulation analysis. Optics Communications. 2021,496, 127125. ( ${ }^{\#}$ Equal contribution)

4. C.J.F. Bertens ${ }^{\#}$, S. Zhang", R.J. Erckens, F.J.H.M. van den Biggelaar, C.A.B. Webers, R.M.M.A. Nuijts, T.T.J.M. Berendschot and M. Gijs, Pipeline for the removal of hardware related artifacts and background noise for Raman spectroscopy. MethodsX, 2020,7,100883. (" Equal contribution)

5. S. Zhang, R.J. Erckens, F. Jongsma, C.A.B. Webers, T.T.J.M. Berendschot*. Dark field illumination in conjunction with confocal Raman spectroscopy for real time noninvasive aqueous humor investigation. Optical Engineering, 2020, 59(9), 092002.

6. C.J.F. Bertens", S. Zhang", R.J. Erckens, F.J.H.M. van den Biggelaar, C.A.B. Webers, R.M.M.A. Nuijts, T.T.J.M. Berendschot and M. Gijs, Confocal Raman spectroscopy: evaluation of a non-invasive technique for the detection of topically applied ketorolac tromethamine in vitro and in vivo. International Journal of Pharmaceutics, 2019,570, 118641. (" Equal contribution)

7. S. Zhang", C.J.F. Bertens", R.J. Erckens, F.J.H.M. van den Biggelaar, C.A.B. Webers, R.M.M.A. Nuijts, T.T.J.M. Berendschot and M. Gijs, In vitro and in vivo datasets of topically applied ketorolac tromethamine in aqueous humor using Raman spectroscopy. Data Brief 2019, 27, 104694. (" Equal contribution)

8. S. Zhang*, R.J. Erckens, F. Jongsma, J. de Brabander, C.A.B. Webers, T.T.J.M. Berendschot. A new Raman spectrometer device for non-invasive determination of the molecular composition of the living eye, Investigative Ophthalmology and Visual Science 58(8), 3112. 2017.

9. S. Zhang, P. Yuan*, J. Yang, Y. Tao, J. Yuan. Optical Absorptivity of Mg-doped ZnO Thin Films Measured by Piezoelectric Photoacoustic Technique. Chinese Journal of Spectroscopy Laboratory, 2011,28(3), 1475-1479.

10. X.Y. Zhou, C.Q. Xiong, S. Zhang, N. Zhang, and Z.X. Nie*. Study of nonlinear resonance effect in Paul trap. J. Am. Soc. Mass Spectrom, 2013, 24,794-800.

11. W.J. Guo, P. Yuan*, J.B. Zhang, S. Zhang. Study on optical absorptivity of ITO thin film by photoacoustic effect. Optical Technique, 2013, 39(3), 273-276.

12. W.J. Guo, P. Yuan*, S. Zhang, W. TAO. Study on Thermal Diffusivity of SrTiO3 Thin Film by Photoacoustic Technique. Piezoelectrics, 2013,35(5),739-742.

13. Z. Li, P. Yuan*, H. Qiao, Y. Guo, S. Zhang. Electron density of laser produced $\mathrm{Cu}$ 
plasma measured by stark broadening of H $\beta$-Line. Journal of Atomic and Molecular Physics, 2010, 27(4), 695-700.

14. P. Yuan*, H. Qiao, M. Su, Z. Li, S. Zhang. Effect of deviation distance to focal spot on spectra of the laser-induced $\mathrm{Cu}$ plasma in air. Journal of Northwest Normal University (Natural Science),2009,45(2),31-34.

15. Shuhe Zhang, T. Berendschot, S. Zhang, Scatting theory for reflective Fourier ptychographic diffraction tomography, arXiv:2008. 2020, 10308.

16. S. Zhang", Q. Qiao", R.J. Erckens, F. Jongsma, C.A.B. Webers, T.T.J.M. Berendschot*. In vitro Raman spectroscopy of sorbitol induced cataracts mimicking diabetic cataracts in porcine lenses. Biomedical Optics Express, 2021. (Submitted) (\# Equal contribution)

\section{Patents}

1. Shuo Zhang, Tos TJM Berendschot, Roel J Erckens, Franciscus Jongsma, John De Brabander. Device for performing measurements of the chemical composition of the anterior eye as well as an integrated optical unit for implementation therein. European Patents (PCT), WO2011089427A1, 2016 November.

2. Zong Xiu Nie, Shuo Zhang, Ning Zhang, Caiqiao Xiong, Lingpeng Zhan, Jing Tian, A planar ion trap mass analyzer. Chinese patent, CN103606509A, 2013.

3. Zong Xiu Nie, Shuo Zhang, Caiqiao Xiong, Xiaoyu Zhou, Jianing Wang, Suming Chen. A new method for manufacturing mass analyzer in mass spectrometers. Chinese patent, CN102623275A, 2012.

4. Zong Xiu Nie, Caiqiao Xiong, Xiaoyu Zhou, Jianing Wang, Rui Chen, Shuo Zhang. A Dual-ion trap mass spectrometer, Chinese patent, CN102290319A, 2011.

5. Zong Xiu Nie, Xiaoyu Zhou, Caiqiao Xiong, Shuo Zhang. An ion transmission heating device dedicated in mass spectrometer, Chinese patent, CN 102324373A, 2011.

\section{Poster}

1. Shuo Zhang, Roel J Erckens, Frans HM Jongsma, John de Brabander, Carroll AB Webers, Tos TJM Berendschot. A new Raman spectrometer device for non-invasive determination of the molecular composition of the living eye. The 2017 annual meeting of ARVO. 2017: Baltimore, USA (B0015)

\section{Book Chapter}

1. Shisong Rong, Shuo Zhang, Jian Li. Chapter: Grasp intelligence for biomedical discovery: an evidence-based approach in What All to Plan Before Starting Biomedical Research: A Handbook on Paperwork, Tools, Techniques, Storage, Banking and Database. (In press) 